\title{
Effect of density stratification on dynamos in gas planets and low-mass stars
}

\author{
Dissertation \\ zur Erlangung des mathematisch-naturwissenschaftlichen Doktorgrades \\ "Doctor rerum naturalium" \\ der Georg-August-Universität Göttingen \\ im Promotionsprogramm PROPHYS \\ der Georg-August University School of Science (GAUSS)
}

vorgelegt von

\section{Rakesh Kumar Yadav}

aus Tohana, Indien

Göttingen, 2014 
Betreuungsausschuss

Prof. Dr. Ulrich R. Christensen

Max-Planck-Institut für Sonnensystemforschung, Göttingen, Germany

Prof. Dr. Ansgar Reiners

Institut für Astrophysik, Universität Göttingen, Germany

Dr. Johannes Wicht

Max-Planck-Institut für Sonnensystemforschung, Göttingen, Germany

Mitglieder der Prüfungskommision

Referent: Prof. Dr. Ansgar Reiners

Institut für Astrophysik, Universität Göttingen, Germany

Korreferent: Prof. Dr. Ulrich Christensen

Max-Planck-Institut für Sonnensystemforschung, Göttingen, Germany

2. Korreferent: Dr. Julien Aubert, CNRS Research Director

Laboratoire de Dynamique des Fluides Géologiques, Paris, France

Weitere Mitglieder der Prüfungskommission:

Prof. Dr. Andreas Tilgner

Institut für Geophysik, Universität Göttingen, Germany

Prof. Dr. Wolfgang Glatzel

Institut für Astrophysik, Universität Göttingen, Germany

Prof. Dr. Manfred Schüssler

Max-Planck-Institut für Sonnensystemforschung, Göttingen, Germany

Prof. Dr. Stefan Dreizler

Institut für Astrophysik, Universität Göttingen, Germany

Tag der mündlichen Prüfung (PhD defence date): 23 Jan., 2015 


\section{"If I have seen further it is by standing on the shoulders of giants."}

Sir Isaac Newton 


\section{Table of Content}

$\begin{array}{ll}\text { Summary } & 5\end{array}$

$\begin{array}{ll}\text { Preamble } & 7\end{array}$

$\begin{array}{lr}\text { Introduction } & 8\end{array}$

$\begin{array}{ll}\text { Paper } 1 & \mathbf{3 1}\end{array}$

Scaling laws in spherical shell dynamos with free-slip boundaries

Paper 2

Consistent scaling laws in anelastic spherical shell dynamos

Paper 3

72

From solar-like to anti-solar differential rotation in cool stars

Paper 4

86

Formation of starspots in self-consistent global dynamo models: Polar spots on cool stars

Conclusions and Outlook

Publications

Acknowledgements

Curriculum Vitae 


\section{Summary}

Under the assumption that a similar dynamo mechanism is responsible for the magnetic field of various solar system planets, many had attempted to derive a predictive scaling law connecting planetary magnetic field and physical properties. However, these early pioneering studies were based on only phenomenological arguments. Drastic assumptions had to be made to tackle the strong non-linearities in the dynamo equations. Without concrete justifications for the underlying assumptions, the various proposed scaling laws remained questionable.

Meanwhile, in the late 1990s, the numerical simulations reached such a level that they started reproducing many observational features of the Earth's magnetic field. This came as a surprise since the numerics was no-way near to modelling the range of length scales required to capture the level of turbulence existing in the Earth's interior. In the following years many parameters studies were conducted to explore what made numerical simulations "Earth like". With such ensemble of numerical simulations it became possible to propose scaling laws and rigorously test them against the simulations. Christensen and collaborators proposed a scaling law connecting the power available through buoyancy forces which was driving the fluid motion and the dynamo generated magnetic field. They successfully tested it on the simulations. The predictions from this scaling law were also in good agreement with the mean magnetic field of the Earth, Jupiter, and some rapidly rotating stars. The agreement with Jovian and stellar magnetic field was rather surprising. The numerical models considered in these studies were incompressible and assumed an overlying mantle (through imposing rigid boundary condition on the velocity), both being suitable for the Earth. The Jupiter (gas planets in general) and stars have no overlaying rocky mantle but rather have free-surface flows and their convection zones are highly compressible. Free-surface flows allow the development of strong zonal flows and the compressibility can act as an additional source of helicity. In principle, both of these factors can substantially affect the dynamo mechanism and can change the various associated scaling laws.

In this cumulative thesis I take a step-by-step approach and progressively include ingredients which are suitable for the convection zones of gas planets and low-mass stars. We begin in Chapter 2 by considering a dynamo model with control parameters similar to the earlier studies but having free-slip velocity boundary condition on the flow to approximately model the free-surface flows in gas planets and low-mass stars. We explore the scaling behaviour of velocity and mean magnetic field and compare it with earlier studies. We find that the nature of the flow is substantially changed but the scaling of the mean magnetic field is similar to what has been reported for dynamos with rigid boundary conditions.

In Chapter 3, I simulate compressible dynamo models by using the anelastic approx- 
imation. I construct an ensemble of compressible dynamo models containing novel simulations and data from earlier studies. I found that once again the major differences were present in the flow properties while the scaling behaviour of the mean magnetic field was very robust. With appropriate averaging of the output mean quantities similar scaling laws can indeed be applied to simulations trying to model the dynamos in Earth, in gas planets and in low-mass stars.

At this juncture, the encouraging results so far indicate that dynamos in planets and stars might behave similarly, to the extent that their mean magnetic fields can be predicted by similar models. This provides a strong motivation to explore what other properties of planets and stars can be considered in a similar manner. In Chapter 4 we analyse new simulations and numerous other past simulations of planetary and stellar convection zones to provide a common framework for the surface differential rotation of gas planets and stars. We show that a single parameter, roughly quantifying the ratio of inertial forces and Coriolis forces, can predict the nature of surface differential rotation. We also analyse the effect of the magnetic field on the nature of the differential rotation.

In Chapter 5, I move further still and conclude this thesis by performing few stateof-the-art numerical simulations with much greater level of complexity. With these simulations, I explore what would happen if an Earth or Jupiter like dynamo with dipoledominated magnetic field were to exist in a "star like" convection zone. The results show that with such a dynamo it is possible to self-consistently produce dark spots near the outer surface of the simulation. The spots were spontaneously produced in the high-latitude regions. These results provide an interesting and plausible explanation for the puzzle of why rapidly rotating stars have dark spots at high latitude. 


\section{Preamble}

This thesis is in a special "cummulative" format. Excluding the chapter Introduction all other chapters are directly reprinted from refereed journal articles which originated through the work performed in this thesis. Each chapter has its own bibliography at the end of the corresponding chapter. Although each of the published articles has its own introduction section, the chapter Introduction provides a general motivation harmoniously connecting the research findings of different chapters of this thesis. 


\section{Introduction}

\subsection{Historical developments}

The advances made in our understanding of the magnetic field generation processes in astrophysical bodies in the second half of the $20^{\text {th }}$ century have been rather remarkable. So much so that it is hard to imagine a time in the past when even the wiser ones commented (Chapman and Bartels 1940) "difficulties which stand in the way of basing terrestrial magnetism on electric currents inside the Earth are insurmountable". Despite the hurdles, we now know that complex enough motions of conducting fluids can produce electric currents which can generate and maintain magnetic fields against ohmic decay (Moffatt 1978). This process, called the 'Dynamo' mechanism, is most probably the reason why magnetic fields are so wide-spread in our universe (Brandenburg and Subramanian 2005).

Through observational evidences we know that the planets (except for Venus) in our solar system either have magnetic fields or had it in the past (Jones 2011). Our Sun has long been known to posses magnetic fields, and strong evidence is building up indicating that probably all stars with convection zones have magnetic fields (Brandenburg and Subramanian 2005). One may wonder how similar are the processes generating magnetic fields in different objects. Discovering correlations between the dynamo generated magnetic field in planets and stars and their physical properties can be a step forward in this regard. For obvious reasons we do not have a direct way of analysing the properties of matter and magnetic field deep inside the planets. Therefore, correlations, if existing, can only be drawn among quantities which are accessible through surface or space observations.

\subsubsection{Heuristic approaches}

Many scientists have tried to discover scaling laws which relate the magnetic fields of various planets and stars with their physical properties. The 'magnetic Bode law', proposed by Blackett (1947), is among the first such attempts. He showed that the ratio of the magnetic moment and the angular momentum of the Earth, the Sun, and that of a recently (at that time) studied star 78 virginis is nearly constant. Later discoveries of magnetic fields in other planets also seemed to closely follow this relation. However, as later pointed out by Cain et al. (1995), the veracity of this law was merely an illusion due to the fact that to calculate magnetic moment and angular momentum one has to multiply the former by $R^{3}$ and the later by $R^{5}$ ( $R$ being the radius of the object). Since these factors span a huge range of values for astrophysical objects a correlation will inevitably be found between 
quantities carrying them in their definition.

With the advent of the homogeneous-dynamo theories based on the magnetohydrodynamic (MHD) principles the focus shifted towards studying physically relevant limits of the governing equations. Since we will be discussing the different force balances of the governing equations it is useful to review them at this stage. We will focus on the Boussinesq limit of the fully compressible MHD equations since historically the former had been employed to make the analytical and the numerical investigations simpler.

To mimic the geometry of convecting regions inside planets and stars a basic setup is assumed which consists of a spherical shell with hotter inner boundary at radius $r_{i}$ and a colder outer boundary at radius $r_{o}$. The first fundamental relation is the mass-continuity relation

$$
\frac{\partial \rho}{\partial t}+\nabla \cdot(\rho \mathbf{u})=0
$$

where $\mathbf{u}$ and $\rho$ are the velocity and the density of the fluid, respectively. Under the Boussinesq approximation the temporal and spacial perturbations in the density are neglected in the equation above. This produces the incompressibility condition

$$
\nabla \cdot \mathbf{u}=0
$$

The next fundamental equation is the Navier-Stokes equation under the Boussinesq approximation which formulates the Newton's second law of motion for the fluid in consideration. In a reference frame rotating with an angular velocity $\Omega$ this equation is given by

$$
\underbrace{\rho \frac{\partial \mathbf{u}}{\partial t}}_{\begin{array}{c}
\text { Acceleration } \\
\text { of fluid }
\end{array}}+\underbrace{\rho \mathbf{u} \cdot \nabla \mathbf{u}}_{\text {Inertial force }}=\underbrace{-2 \rho \mathbf{\Omega} \times \mathbf{u}}_{\text {Coriolis force }} \underbrace{-\nabla P}_{\begin{array}{c}
\text { Force due to } \\
\text { pressure gradients }
\end{array}}+\underbrace{\alpha \rho \mathbf{g} T^{\prime}}_{\begin{array}{c}
\text { Thermal buoyancy } \\
\text { force }
\end{array}}+\underbrace{\mathbf{B}}_{\begin{array}{c}
\text { Lorentz force } \\
\mathbf{J} \times \mathbf{B}
\end{array}}+\underbrace{\nu \nabla^{2} \mathbf{u}}_{\text {Viscous force }},
$$

where $P$ is pressure, $\alpha$ is the coefficient of thermal expansion, $\mathbf{g}$ is the gravity (pointing radially inwards), $T^{\prime}$ is temperature perturbation, $\mathbf{J}$ is the current, $\mathbf{B}$ is the magnetic field, and $v$ is the shear viscosity. Note that the essence of the Boussinesq approximation appears in the thermal buoyancy force term where the changes in density due to temperature perturbations are kept to allow the possibility of convection. Furthermore, the viscous force term is also simplified as compared to its more generic tensorial form and contains only a Laplacian operator.

\section{Geostrophic balance}

In the past, the Geodynamo was assumed be the archetype of planetary dynamos (due to the lack of observational constraints about other planets) and ideas applied to it were directly extended to other planets. Busse (1975) was among the first to theoretically explore the nature of the Earth's magnetic field from the perspective of convection-driven dynamos. The idea of Earth's core convection strongly influenced by the Coriolis forces 
due to Earth's rotation was of central importance in his analysis ${ }^{1}$. He also tried to incorporate some aspects of the Earth's spherical geometry by considering a cylindrical annulus with sloping top and bottom boundaries. In the limit of rapid rotation (i.e. large Coriolis forces), steady flow, vanishing magnetic field, and small viscosity, all but four terms vanish in Eqn. 1.3. If we further assume that the non-linear term $(\rho \mathbf{u} \cdot \nabla \mathbf{u})$ and the buoyancy force are of secondary importance, then we arrive at the "Geostophic" force balance, where the Coriolis force is balanced by the pressure gradients. This force balance is the basis of the famous Taylor-Proudman theorem which shows that motions in such systems will be confined to directions perpendicular to the rotation axis. Fritz Busse assumed that to the leading order this Geostrophic force balance is valid in the Earth liquid core. Busse (1976) carried this assumption to the planetary dynamos and proposed a scaling law for the mean magnetic field for different planets. He proposed that the second leading order force balance is between the inertial force term $\left(\approx \rho u^{2} / r_{o}\right.$, approximating $\nabla$ with $\left.1 / r_{o}\right)$ and the Lorentz force term $\left(\approx B^{2} / r_{o}\right.$, assuming $J$ roughly scales as $\left.B / r_{o}\right)$. Equating these two forces, and assuming that the velocity scales as $\Omega r_{o}$, we arrive at Busse's scaling law

$$
B^{2} \propto \rho \Omega^{2} r_{o}^{2}
$$

\section{Magnetostrophic balance}

Stevenson (1979) approached the problem of planetary and stellar convection from a more idealized perspective. He developed a theory for rotating and magnetic convection in different limits in a planar geometry. His main aim being to provide an alternative to the mixing-length-theory (MLT) when rotation and magnetic fields significantly affect the convection. Convection transfers heat from one place to another. For a given set of control parameters, convection prefers least temperature gradients which drives the convection itself such that a certain amount of heat is continuously transferred from hotter to colder regions. If we disturb the system from this state it will either invigorate or dampen convection (depending on how we disturbed it) in order to reach the 'optimal' state again. Given a certain rotation speed and magnetic field strength Stevenson (1979) sought to find 'optimal' magnetic field strengths in the limit of rapid rotation such that the temperature gradients are minimized. Stevenson (1983) writes in a later review that "once the criterion for dynamo onset is satisfied, the field begins to grow. The Lorentz force relaxes the constraint on the convection imposed by the Coriolis force and causes the convection to become more vigorous. The system continues unstably until it encounters a new stable state at or near the 'optimal' field". In the limit of ohmic diffusion overwhelming other diffusion processes ${ }^{2}$ a parameter called the Elsasser number $\Lambda$, defined as the ratio of the Lorentz force ${ }^{3}\left(\sigma u B^{2}\right)$ and the Coriolis term $(\rho \Omega u)$ in Eqn. 1.3, is of importance and the

\footnotetext{
${ }^{1}$ Even assuming the mixing-length-theory relation velocity $\propto$ (Heat-Flux/density $)^{1 / 3}$, which is very likely an overestimate for rapidly rotating systems, the fact that heat-fluxes from planetary cores are small and the density is large results in a rather moderate flow velocity of the order of a few $\mathrm{cm} / \mathrm{s}$. This means that even moderate rotation rate in planets will have a prominent effect on core convection.

${ }^{2}$ Physically relevant limit since magnetic diffusivities are much larger than thermal or viscous ones in planetary and stellar convection zones.

${ }^{3}$ Assuming $J$ scales as $\sigma u B$, where $\sigma$ is electrical conductivity.
} 
'optimal' state discussed above is reached when it is close to unity, i.e.

$$
B^{2} \propto \frac{\rho \Omega}{\sigma} .
$$

The condition of $\Lambda \approx 1$ is known as the "magnetostrophic" force balance.

Curtis and Ness (1986) considered the same magnetostrophic force balance as discussed above but assumed that the current $J$ scales as $B / r_{o}$. Unlike the case discussed above where mean velocity $u$ cancels on both sides in the force balance (due to the choice of $J \propto \sigma u B$ ), here $u$ stays in the force balance. Therefore, relating mean convective velocities to some physical parameters was required. Busse (1975)'s assumption of equating mean convection velocity with rotational velocity was questioned, and, instead, they assumed that "the energy flux associated with the core convection velocity can be scaled directly from the observed heat flux, $F$, whose origin lies in the internal heat source of the planet." With this assumption in mind, they used the classical MLT prediction of $u \propto(F / \rho)^{1 / 3}$ for the velocity and obtained

$$
B^{2} \propto \rho^{2 / 3} \Omega F^{1 / 3} r_{o}
$$

To discuss the next approach we first need to familiarize ourselves with the fundamental equation governing the magnetic field evolution. The magnetic induction equation derived using the Maxwell's equations, under the assumption that the displacement currents in the Ampere's circuital law are negligible and the Ohm's law is valid, is

$$
\frac{\partial \mathbf{B}}{\partial t}=\underbrace{\nabla \times(\mathbf{u} \times \mathbf{B})}_{\text {Magnetic advection }}+\underbrace{\lambda \nabla^{2} \mathbf{B}}_{\text {Magnetic diffusion }},
$$

where $\lambda$ is the magnetic diffusivity. Backus (1958) analytically showed that the ratio of the advection and the diffusion term in Eqn. 1.7, i.e. $u r_{o} / \lambda$, has to be greater than or equal to $\pi$ for a dynamo to exit in a constant electrical conductivity sphere. Mizutani et al. (1992) build up on this result and also considered that $u \propto \Omega r_{o}$ is probably too large (Busse 1976). With $\lambda / r_{o}$ and $\Omega r_{o}$ as rough lower and upper bounds, respectively, Mizutani et al. (1992) took a rather "engineering" approach and proposed that the velocity scales according to the geometric mean of the two limits, i.e. $u \approx(\lambda \Omega)^{1 / 2}$. With this velocity scaling, and again assuming the magnetostrophic force balance, we arrive at

$$
B^{2} \propto \rho \Omega^{3 / 2} \lambda^{1 / 2} r_{o}
$$

A somewhat different approach was adopted by Sano (1993). Instead of starting from the fundamental equations (Eqns. 1.3 and 1.7), he used the mean-field MHD equations (Krause and Rädler 1980) where velocity and magnetic field were decomposed into mean and fluctuating parts. He assumed the classical " $\alpha \omega$ " dynamo mechanism ${ }^{4}$, along with the assumption of a magnetostrophic force balance. After proposing a chain of arguments, involving the nature of turbulent fluctuations and the order of magnitude of various terms and quantities, he finally arrived at

$$
B^{2} \propto \rho \Omega^{2} r_{o}
$$

\footnotetext{
${ }^{4}$ Toroidal magnetic field was produced by the axisymmetric toroidal flows and the poloidal field was produced by small scale helical turbulent fluctuations.
} 
Note that it is very much similar to Busse (1975)'s proposed scaling law (Eqn. 1.4), except for a more moderate dependence on the radius of the convection zone. The advantage of this analysis was that the mean-field equations allowed Sano to consider the back-reaction of magnetic field on the flow, at least under the framework of the mean-field dynamos.

The computing power was growing exponentially in the second half of the 20th century. Gilman (1977) was among the early pioneers who exploited the power of the computers to model fully non-linear convection using fundamental equations of motion in rotating spherical shells. However, it was only after the remarkable results by Glatzmaier and Roberts (1995a,b), Kageyama and Sato (1995), which showed that computer simulations can reproduce some of the observed properties of the Geodynamo with striking details, that the field of numerical dynamo simulations was firmly established.

\section{MAC balance}

In light of the new results from the numerical dynamo simulations, Starchenko and Jones (2002) considered that except for the viscous and the inertial force terms, other forces involved in Eqn. 1.3 are of similar order of magnitude. They termed this triple force balance as "MAC" balance, after Magnetic, Archimedian, and Coriolis. They first constrained the velocity scaling by equating the Coriolis and the buoyancy forces. For the magnetic field, Lorentz force and the Coriolis force were compared. With these comparisons, mean magnetic field roughly scales as

$$
B^{2} \propto \rho(\Omega E)^{1 / 2} r_{o}^{3 / 2} .
$$

One notable novelty of Starchenko and Jones (2002)'s work was that they considered the so called "Anelastic" approximation rather than the Boussinesq approximation (Eqn. 1.3) employed in earlier work; we will come back to the anelastic approximation in later discussion.

One may rightly ask how can there be so many scaling predictions of magnetic field for our planets in the solar system? It is evident from the preceding discussion that the scaling laws discussed so far have been based on heuristic arguments, frequently simplifying things for the sake of making analytical treatment tractable at the expense of sacrificing relation with nature. With these simplifications one can only roughly compare the order of magnitudes of prediction and observation. Moreover, as was the case with magnetic Bode law, many of the scaling laws were applied to the planets not in the form of magnetic field predictions but rather in the form of magnetic moment predictions. This practice of comparing magnetic moments (carrying a cube-of-radius factor) can lead to artificial correlations since both right and left had side of the scaling law will be carrying largerange quantities with different exponents (Cain et al. 1995).

\subsubsection{Parameter studies}

Although the numerical reproduction of some observable traits of the Earth's magnetic field was fascinating, scepticism was nonetheless prevalent in the community about the results. The reservations were motivated by the fact that the numerical dynamo simulations (even in simple Cartesian boxes) were not even close to modelling the vast range of length scales present in the real flows in laboratory, let alone the flows in the planetary 
interiors. Running computer simulations at drastically modified control parameters is the primary reason why simulations are far removed from reality. For instance, viscosity in planetary and stellar convection zones is supposedly extremely small. Such small viscous drags allow motions to exist not only at large scales but also at very small scales. For technical reasons, we can not use a very small viscosity in numerical simulations since that would require a numerical grid resolution which can capture large as well as very small length scales, i.e. too many grid points. Hence, viscosity is artificially enhanced to suppress the small scale motions and in turn help alleviate the need to have very small grid resolution.

Although we can not reach extreme control parameter regimes, we can at least try to figure out if and by how much do the control parameters affect the final results of numerical simulations. Christensen et al. (1999) carried out foundational work in this regard and systematically studied the influence of various control parameter on the convection driven dynamos in rotating spherical shells. Many studies in later years followed suit and with-in few years compiled a rich variety of dynamo results in different contexts.

\section{Scaling laws in simulations}

With the luxury of having an ensemble of numerical dynamo simulation results one can do something rather unprecedented: propose/infer suitable force/energy balances for these models, extract the corresponding scaling laws, and check the veracity of these predictions against the set of numerical simulations. Christensen and Aubert (2006) followed this strategy and brought about nothing less than a paradigm shift in the area of scaling laws for magnetic field. Since checking the ideas put forth in that study constitutes a major portion of this thesis we will discuss them in more details.

We shall now introduce the non-dimensional form of the fundamental equations (Eqns. 1.3 and 1.7) which have been employed by Christensen and Aubert (2006) in their analysis. Choosing the shell thickness $D=r_{o}-r_{i}$ as the length scale, inverse rotation frequency $\Omega^{-1}$ as the time scale, temperature contrast $\Delta T$ between the top and the bottom boundary as the temperature scale, and $\Omega d \sqrt{\rho \mu}$ as the magnetic field scale, the non-dimensional forms of Eqns. 1.3 and 1.7 are

$$
\begin{gathered}
\frac{\partial \mathbf{u}}{\partial t}+\mathbf{u} \cdot \nabla \mathbf{u}=-2 \hat{z} \times \mathbf{u}-\nabla P+R a^{*} \frac{\mathbf{r}}{r_{o}} T^{\prime}+\mathbf{J} \times \mathbf{B}+E \nabla^{2} \mathbf{u}, \\
\frac{\partial \mathbf{B}}{\partial t}=\nabla \times(\mathbf{u} \times \mathbf{B})+\frac{E}{P m} \nabla^{2} \mathbf{B} .
\end{gathered}
$$

Since the density of the outer liquid core of the Earth is nearly constant the gravity in the earlier models was assumed to vary linearly with radius, i.e. $\mathbf{g}=g_{o} \mathbf{r} / r_{o}$ where $g_{o}$ is the gravity at the outer boundary. Note that we are using the same symbols for dimensional and non-dimensional velocity, magnetic field, and other quantities for the sake of brevity. Important non-dimensional numbers appear after this exercise:

$$
\begin{gathered}
\text { modified Rayleigh number } R a^{*}=\frac{\alpha g_{o} \Delta T}{\Omega^{2} D}, \\
\text { Ekman number } E=\frac{v}{\Omega D^{2}}, \\
\text { magnetic Prandtl number } P_{m}=\frac{v}{\lambda} .
\end{gathered}
$$


The conventional numerical simulations also solve the (non-dimensional) equation describing the temperature evolution

$$
\frac{\partial T}{\partial t}+\mathbf{u} \cdot \nabla T=\frac{E}{P r} \nabla^{2} T
$$

where $\operatorname{Pr}=v / \kappa$ ( $\kappa$ being the thermal diffusivity) is the classical Prandtl number. As has been the case so far, this equation will not be of much importance for our subsequent discussion as well.

The first assumption in the analysis of Christensen and Aubert (2006) is "... that the magnetic field strength is not determined by a force balance, but by the power available to balance Ohmic dissipation". In other words, they considered the balance of input and output energy as the guiding principle to determine the strength of the magnetic field. In a numerical dynamo simulation energy input via thermal buoyancy mechanism (including others, for example, mechanical forcing or precession) is eventually dissipated through viscous and ohmic diffusion. Let us denote $P$ as the input power which is defined as

$$
P=R a^{*} \int g u_{r} T^{\prime} d v
$$

with $\int d v$ being the volume integral. This power is eventually lost in the form of viscous dissipation rate $D_{v}$ and ohmic dissipation rate $D_{\lambda}$, i.e.

$$
P=D_{v}+D_{\lambda}
$$

If $f_{\text {ohm }}$ is the fraction of power lost in the form of ohmic dissipation, then

$$
f_{\text {ohm }}=\frac{D_{\lambda}}{P},
$$

where

$$
D_{\lambda}=\frac{E}{P m} \int(\nabla \times \mathbf{B})^{2} d v .
$$

Let us also define an Ohmic dissipation time

$$
\tau_{m a g}=\frac{E_{m a g}}{D_{\lambda}}
$$

which is the characteristic time-scale associated with ohmic dissipation, here $E_{\text {mag }}$ is the total magnetic energy in the shell. Using a set of numerical dynamo simulations, Christensen and Tilgner (2004) showed that $\tau_{m a g}$ scales as $1 / R o$, where $R o$ is the Rossby number ${ }^{5}$. This relation becomes a little more intuitive if we express the time not in terms of $\Omega^{-1}$ but rather in terms of $D^{2} / \lambda$ (i.e. magnetic diffusion time scale). This relation then becomes $\tau_{m a g} \propto R m^{-1}$. It can be expected that the time at which magnetic energy dissipates scales with the magnetic Reynolds number $R m$; higher the $R m$ shorter will be the typical length scale of the magnetic field and hence faster will be its dissipation process.

\footnotetext{
${ }^{5}$ Note that in our non-dimensional units $R o$ is actually the non-dimensional velocity defined by $u(\Omega D)^{-1}$ where $u$ is the dimensional velocity.
} 
Using the definition and scaling behaviour of $\tau_{m a g}$ we can write

$$
\frac{E_{\text {mag }}}{D_{\lambda}} \propto \frac{1}{R o} \rightarrow \frac{E_{\text {mag }}}{f_{\text {ohm }}} \propto \frac{P}{R o} .
$$

In our non-dimensional units Lorentz number $L o$ quantifies the mean magnetic field density in the spherical shell, i.e. $L o=\sqrt{2 E_{m a g} / V}$ ( $V$ being the volume of the spherical shell). We can then write

$$
\frac{L o}{\sqrt{f_{\text {ohm }}}} \propto \sqrt{\frac{P}{R o}} .
$$

Christensen and Aubert (2006) showed that to very good approximation

$$
P \propto R a^{*}(N u-1) \frac{E}{P r}=R a_{Q}^{*}
$$

where $R a_{Q}^{*}$ is an advected heat-flux based Rayleigh number and $N u$ is the conventional Nusselt number defined by the ratio of the total heat flux (advected+conducted) to the conducted heat flux on the outer boundary ${ }^{6}$. With this approximation we can finally write

$$
\frac{L o}{\sqrt{f_{\text {ohm }}}} \propto \sqrt{\frac{R a_{Q}^{*}}{R o}} .
$$

For the velocity $(R o)$ scaling Christensen and Aubert (2006) took an unconventional approach and instead of proposing a force balance argument they used the best-fit scaling prediction from their simulation data. The figure representing the scaling of $R o$ (recall that in our units non-dimensional velocity is $R o$ ) is shown in the left panel of Fig. 1.1. The best-fit power law (solid line in the figure) describing the data is $R o=0.85\left(R a_{Q}^{*}\right)^{0.41}$. Using this scaling and Eqn. 1.22 we can predict that

$$
\frac{L o}{\sqrt{f_{\text {ohm }}}} \propto\left(R a_{Q}^{*}\right)^{0.3}
$$

This relation constitutes the power-based scaling law for the magnetic field. The right panel in Fig. 1.1 shows $L o / \sqrt{f_{\text {ohm }}}$ as a function of $R a_{Q}^{*}$ for the same set of simulations. The best-fit scaling law obtained from this data-set is

$$
\frac{L o}{\sqrt{f_{\text {ohm }}}} \propto\left(R a_{Q}^{*}\right)^{0.34}
$$

which shows reasonably good agreement with the prediction given in Eqn. 1.23.

Interestingly, the empirical observation $R o=0.85\left(R a_{Q}^{*}\right)^{0.41}$ agrees with data from a somewhat related setup. Aubert et al. (2001) carried out a thorough experimental study of rotating convection in spherical shells. These experiments were purely hydrodynamic,

\footnotetext{
${ }^{6}$ Computation of $R a_{O}^{*}$ is less demanding as compared to $P$ as the former involves a surface integral (via the calculation of $\mathrm{Nu}$ ), while the latter involves a volume integral. However, $P$ is a more useful quantity for applying the scaling laws to observations since the heat-flux coming out from planets and stars is a measurable quantity and $P$ and the observed heat-flux are related.
} 

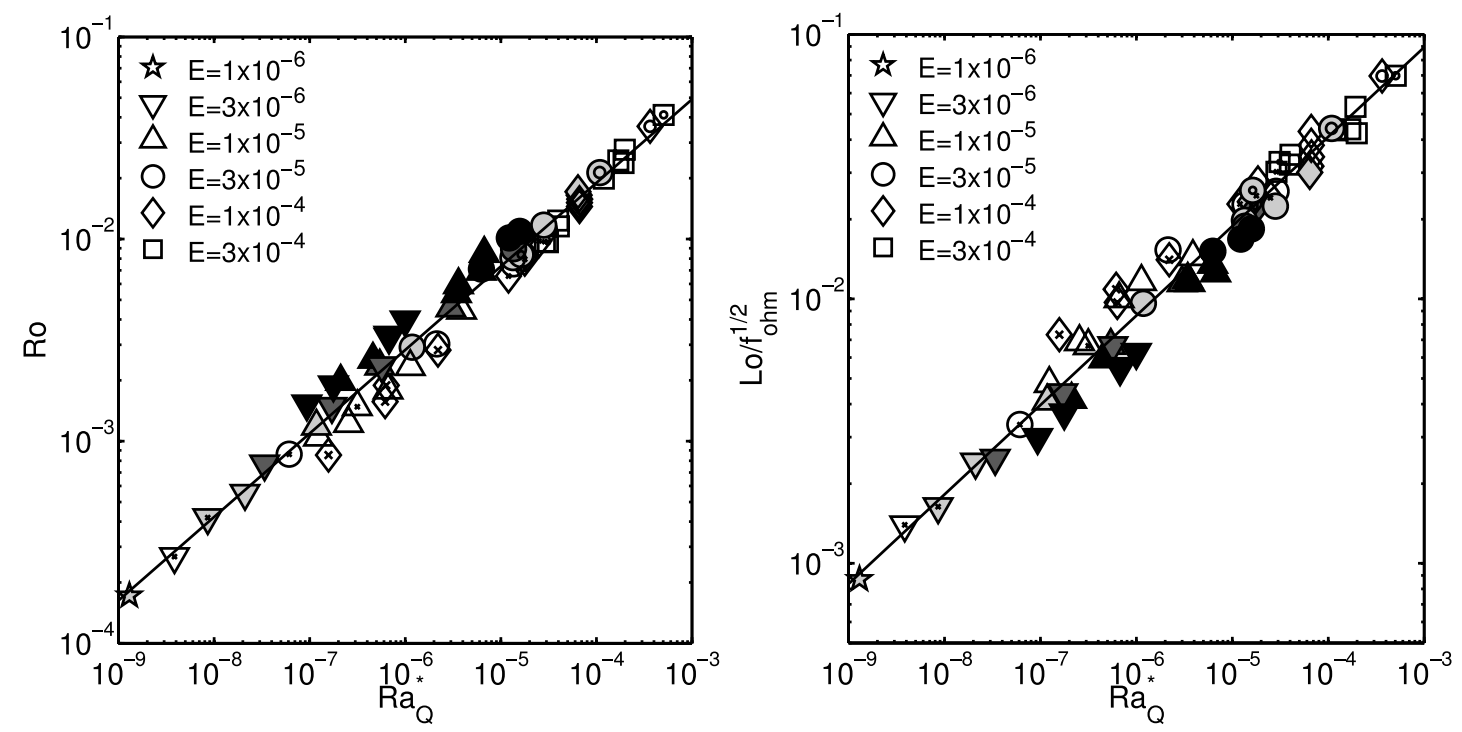

Figure 1.1: Scaling behaviour of $R o$ and $L o$ as a function of $R a_{Q}^{*}$ for a set of numerical dynamo simulations. Figure adapted from Christensen and Aubert (2006).

and the gravity was modelled with centrifugal forces. To explain the results of this experiment they considered the equation for vorticity $\nabla \times \mathbf{u}$, which is obtained by taking the curl of Eqn. 1.3 (without magnetic fields). They assumed similar order-of-magnitude for terms originating from the Coriolis, the inertia, and the buoyancy (Archimedean) forces. The resulting 3-term balance is frequently referred to as the CIA balance. This balance results in a velocity scaling $R o \propto\left(R a_{Q}^{*}\right)^{2 / 5}$, in close agreement with what Christensen and Aubert (2006) found empirically for their dynamo models. However, Christensen and Aubert (2006) argue that the CIA balance is not suitable for their dynamo simulations since it does not involve the Lorentz force term which is very important in the dynamo simulations. Indeed, it is the Lorentz force which has to become active to stop the exponential growth of the dynamo. Hence, it seems unlikely that the considerations application to the hydrodynamic simulations would apply to the dynamos as well.

\section{The devil is in the details}

So far we have not discussed one of the most unpleasant aspect of the numerical dynamo simulations. Unlike the case of fluids in planetary and stellar convection zones where the diffusive transport coefficients are very small, the diffusion coefficients in simulations are amplified by orders of magnitude for technical reasons. To provide some perspective, the Ekman number $E$ for Earth is about $10^{-15}$ and the magnetic Prandtl number $P_{m}$ is about $10^{-6}$, while numerical modellers typically use $E=10^{-4}-10^{-6}$ (record low $10^{-7}$ ) and $P_{m}=5-0.5$ (record low $\approx 0.05$ ). Due to this the diffusion term in Eqn. 1.11 is not as small as it is supposed to be in planets and stars, and it can have substantial influence on the final dynamo solution.

Is it then justified to extend the scaling laws derived from the numerical simulations to natural dynamos? The most optimistic scenario is that numerical dynamo simulations are capturing the essence of natural systems by only modelling the physics happening at 
large scales. Small-scale turbulence, which can not be modelled with current simulations, only helps to dissipate energy and is not very important for things at large scales. This scenario will support the notion of similar "asymptotic states" for numerical simulations and natural systems, and, if so, then scaling laws from simulations should be relevant for natural dynamos. But since numerical simulations are still "polluted" by diffusion at large scales, the "devil" in the details can not be avoided. The question, then, is to what extent the scaling laws are affected by the diffusive processes.

Indeed, when Christensen and Tilgner (2004) derived the scaling relation for $\tau_{m a g}$ they found that the scatter of data from numerical models can be significantly reduced if $P_{m}$ (quantifying the ratio of two diffusive processes) is introduced as another fitting parameter. Similar improvement in the fit-quality was reported by Christensen and Aubert (2006). Furthermore, Soderlund et al. (2012) found that viscous forces are influencing the large scale magnetic fields in contemporary geodynamo simulations and King and Buffett (2013) explored scaling laws based on balancing viscous forces with other forces mentioned above. It is clear that the diffusion terms in Eqns. 1.11 and 1.12 are influencing the output of the numerical simulations.

Christensen and Tilgner (2004) compared both of their scaling laws for $\tau_{m a g}$, i.e. with/without $P_{m}$ dependence, against the Karlsruhe dynamo experiment (Müller and Stieglitz 1999). The scaling law carrying no $P_{m}$ dependence agreed much better with the experimental data and the prediction agreed within the $3 \sigma$ bounds of deviations. Similar thing happened when Christensen and Aubert (2006) checked the prediction for magnetic field based on the scaling laws with/without $P_{m}$ dependence against the Earth's magnetic field. Based on this analysis it has been argued that the $P_{m}$ dependence of scaling laws in numerical models is due to the fact that the value of $P_{m}$ is so high in simulations. As far as the dependence of mean magnetic field on the available power (or $R a_{Q}^{*}$ ) is concerned, the numerics is already in a state similar to the ones present in the natural dynamos. The expectation is that as we approach towards lower and lower $P_{m}$ values ${ }^{7}$, the contribution of the diffusive processes will becomes smaller and smaller, and eventually simulations will reach an asymptotic state where the scaling laws will become independent of the value chosen for $P_{m}$.

\subsection{From simulations to planets and stars}

Through satellite observations the order-of-magnitude of the magnetic field in our solar system planets is reasonably well constrained. Our Sun also possesses a magnetic field. On the large scale its magnetic field is dipolar which reverses its direction almost regularly. On the smaller scales, Sun's magnetic field shows complex behaviour and forms active regions. The strongest field on the Sun exists in such active regions and reaches 1000s of G. The dipolar field is much weaker with field strength of about $10 \mathrm{G}$ (Solanki et al. 2006). Sun rotates once in about 25 days, and considering the Sun as a member of stellar population, it is a rather slowly rotating star.

To infer the magnetic field in other stars the Zeeman splitting method can be used. However, as Reiners (2012) notes "... in a measurement of Zeeman splitting in Stokes I one faces the difficulty to disentangle the effect of Zeeman broadening from all other

\footnotetext{
${ }^{7}$ Indirectly, it also requires lower $E$ and higher $R a$ which is the physically relevant regime.
} 
broadening agents. This requires precise knowledge of the spectral line appearance in the absence of a magnetic field. This task requires extremely good knowledge about spectral line formation, velocity fields, and the temperature distribution on the star". It means that this method has rather limited scope.

Fortunately, there are indirect methods to infer the presence of stellar magnetic fields. For the Sun, there is a strong positive correlation between its X-ray luminosity $\left(L_{X}\right)$ and its cyclic magnetic fields. Such a positive correlation is brought about via many heating mechanisms related to the magnetic field. Assuming that similar correlation applies to other stars, high X-ray luminosity (normalized by total bolometric luminosity $L_{b o l}$ ) would imply strong magnetic fields. X-ray luminosities of a number of stars have been observed and the famous "Rotation-activity" relation has been empirically inferred based on this data. Pizzolato et al. (2003) used $R_{e}$, defined as the ratio of the stellar rotation period and the typical convective turn-over time in the convection zone, to quantify the effect of rotation on the convection. Note that this is a simplified version of the Rossby number $R o$ parameter defined earlier. They found two distinct regimes: for $R_{e}>0.1$ the ratio $L_{X} / L_{b o l}$ increases for decreasing $R_{e}$ and for $R_{e}<0.1$ the ratio $L_{X} / L_{b o l}$ saturates to a plateau. Roughly speaking, this implies that stars with faster rotation velocities generate stronger magnetic fields but this trend breaks down below a certain rotation period, after which stars with faster rotation have more-or-less similarly strong magnetic fields. In this "saturated" regime stars possess rather strong large-scale magnetic fields which are of KG level, roughly two orders-of-magnitude larger than the large-scale solar dipolar field.

Many of the stars in the regime where $L_{X} / L_{b o l}$ levels off are young T Tauri stars and low-mass M-dwarfs. Fortunately, the large-scale magnetic field morphology of some of these stars have been mapped using the Zeeman-Doppler-Imaging (ZDI) technique (Semel 1989). Quite frequently, the magnetic field in such stars carries a strong dipolar component, much like the magnetic field in the Earth (see e.g. Donati and Landstreet 2009). Under the light of this Rotation-activity relation and the frequent occurrence of strong dipolar fields, it has been argued that below a certain rotation period stars might be harbouring a different type of dynamo (compared to the Sun).

Christensen et al. (2009) followed this line-of-thought and tried to connect the dipoledominated planetary dynamos in our solar system to the low-mass rapidly-rotating stars. Remarkably, they demonstrated that scaling laws inferred from "Earth like" dynamo models were capable of predicting the mean magnetic field in rapidly-rotating stars as well. The figure demonstrating this comparison is reproduced in Fig. 1.2. They also showed that stars with slower rotation periods consistently produce weaker magnetic fields than that predicted from the scaling law, implying that slower rotation induces less efficient dynamos. These results excite the following idea: as long as a star rotates fast enough and has a deep convection zone, the resulting dynamo generated in the interior is very much akin to the one operating in the interior of rapidly-rotating planets. Given this similarity, it would then be expected that similar scaling laws might apply to both. Moreover, the fact that slowly rotating stars do not follow the scaling law also implies that the dynamo mechanism is probably fundamentally different in them. 


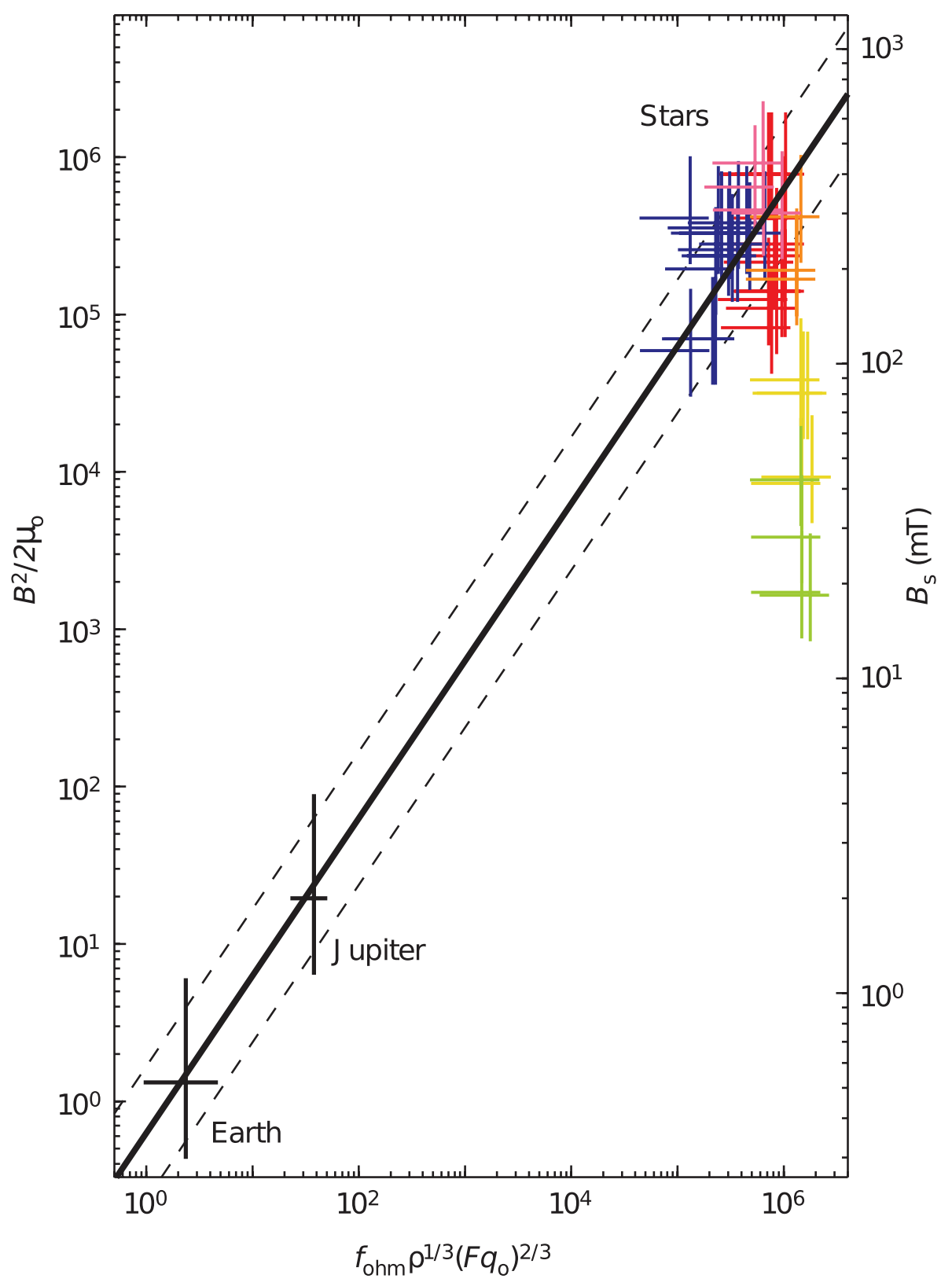

Figure 1.2: Comparison of prediction of magnetic field from scaling law based on Eqn. 1.24 with stars and planets. The factor $f_{\text {ohm }}$ was assumed to be unity for natural objects; $\rho$ is the density of the objects, $F$ is an "efficiency factor" defined to take into account the density stratification effects in a simplified manner, and $q_{o}$ is the observed bolometric heat-flux. The coloured data represent different type of stars: blue for young $\mathrm{T}$ Tauri stars, red and pink for old M dwarfs, and (green, yellow, orange) for solar mass stars with rotation period ( $>10$ days, between 4 and 10 days, $<4$ days), respectively. The solid line is prediction from dynamo simulations resembling Earth (i.e. dipole dominated magnetic field) and the dashed line are $3 \sigma$ deviation bounds. The figure is adapted from Christensen et al. (2009). Note that the figure has been modified for the sake of clarity. 


\subsubsection{Focus of Chapter 2}

Along with being exciting, the good agreement between magnetic field predicted from power-based scaling law and the magnetic field observed in gas planets and stars was rather puzzling. The numerical scaling laws discussed so far have been inferred from dynamo simulations whose basic setup is inspired by the Earth's properties. First important consideration in these simulations is the assumption of an overlaying mantle which is modelled via assuming a no-slip or rigid boundary condition for the velocity on the outer boundary of the shell. Through systematic parameter studies (Christensen 2002, Aubert 2005) we know that boundary conditions can have a rather drastic impact on the flow structure in rotating spherical shells. Most notably, if, instead of imposing rigid boundary conditions we impose a free-slip or stress-free boundary condition (appropriate for gas-planets and stars), then strong differential rotation is excited, much like the azimuthal jets visible on the surface of gas-planets. Since differential rotation can have a significant impact on the dynamo mechanism via the classical " $\Omega$-effect" (generation of toroidal magnetic fields through winding up of the poloidal field lines), there is no reason to believe that the scaling laws derived from models with rigid-boundary conditions should also be used to predict the magnetic field in gas-planets and stars.

The second chapter of thesis is dedicated to analysing such aspects. To isolate the effects of boundary condition on the different scaling laws I will keep the simulation setup very much similar to that of Christensen and Aubert (2006) but change the velocity boundary condition to free-slip. As discussed in that chapter, this change of boundary conditions mainly affects the velocity scaling behaviour and the scaling of magnetic field in not substantially changed. I will discuss how initial condition impacts the final state of the simulations and how dipole-dominated dynamos consistently generate substantially stronger magnetic fields than those dynamos where dipole component is very weak.

\subsubsection{Focus of Chapter 3}

The second aspect where Earth's fluid core fundamentally differs from that of gas-planets and stars is the degree of density stratification: the density changes only by $\approx 20 \%$ in the Earth's fluid core while it changes by orders-of-magnitude in gas-planets and stars. As mentioned earlier, the numerical models discussed so far employ Boussinesq approximation (justifiably so) to model the Earth's fluid core. But this approximation is certainly more debatable for gas-planets and stars where density varies a lot. One potential direct connection between density stratification and the dynamo mechanism is through helicity $\mathbf{u} \cdot(\nabla \times \mathbf{u})$ (a measure to quantify the helical nature of flow). Helicity is thought be a good proxy for the classical " $\alpha$-effect" in the classical mean-field MHD formulation which converts toroidal field to poloidal field. If we take the curl of Eqn. 1.3, then we obtain the evolution equation of the vorticity $\nabla \times \mathbf{u}$. Of particular importance is the coriolis term $\nabla \times \mathbf{u} \times \boldsymbol{\Omega}$ whose expansion contains a term $\boldsymbol{\Omega}(\nabla \cdot \mathbf{u})$. This term is no-longer zero in compressible fluids since $\nabla \cdot \mathbf{u}$ is not zero any more. This "compressional torque" term introduces vorticity through the expansion and contraction of fluid parcels (Glatzmaier et al. 2009, Reshetnyak 2012). Depending on how these additional vortical structure move they can act as an additional source of helicity in compressible systems which was missing in Boussinesq fluids. 
Ideally one would like to use the fully compressible MHD equations to model such convection zones. However, the drawback of such an approach is that these equations allow the existence of acoustic-waves whose time scale of evolution would be much smaller than the important time scales in dynamo simulations, e.g. convective turn-over time scale and magnetic diffusion time scale. To model such a system in its entirety a simulation should resolve the fast acoustic time scale as well as other slower time scales. This imposes a prohibitively small time-step on the time evolution algorithms. Since we need to solve thousand of convective turn-over times in a typical dynamo simulation such a small time-step would increase the simulation run-time by orders of magnitude. Global dynamo simulations are very time consuming to begin with, and fully compressible approach would be a completely un-affordable "overkill".

To avoid this problem the "anelastic" approximation has been introduced. Originally developed in the atmospheric community (Batchelor 1953, Ogura and Phillips 1962) this approximation has since been extensively used in the studies of density stratified convection zones in planets and stars. A detailed discussion and derivation of the anelastic approximation is beyond the scope of this thesis. Readers who are interested in the intimate details are referred to Gilman and Glatzmaier (1981), Braginsky and Roberts (1995), Lantz and Fan (1999), Jones et al. (2011). Even more details can be found in DeRosa (2001). In the following we briefly discuss some of the essential and interesting aspects of this approximation.

The first basic assumption in this approximation is the notion that the sub-sonic convection in the interiors of planets and stars introduces fluctuations in the thermodynamic quantities which are small as compared to a "well mixed" background state (i.e. the adiabatic state). In this spirit, the thermodynamic variables are written as a sum of static background values and small time-dependent perturbations, i.e. $\zeta(x, t)=\zeta_{o}(x)+\epsilon \zeta_{1}(x, t)$, where $\zeta$ is some thermodynamic quantity, $\epsilon$ is a small number, and $x, t$ are spacial and temporal coordinates. In spherical geometry the background state values are a function of radius alone. With this assumption, the MHD equations now have two parts: zero-order time-independent background part and a higher order time-dependent part.

To leading order, the mass-conservation equation becomes

$$
\nabla \cdot\left(\rho_{o} \mathbf{u}\right)=\mathbf{0} .
$$

As oppose to the incompressibility relation in the Boussinesq approximation, here, it is the mass-flux $\rho_{o} \mathbf{u}$ which is divergence less. Absence of an explicit time-derivative of the density again allows us to filter out acoustic waves. However, note that through changes in other thermodynamic variables small density perturbations do exist, although they occur on a slow convective time scale. Another novel aspect of the anelastic approximation considered in this thesis is that the conduction of heat is assumed to be proportional to entropy gradients (other variants might assume differently), unlike the Boussinesq approximation where temperature gradients derive conduction. Furthermore, Lantz and Fan (1999) note that the most appropriate background state in the system under consideration is an isentropic one, i.e. entropy is assumed constant in the background state. It is also noteworthy that viscous heating and Joule heating do not appear in the Boussinesq systems while they do appear in the anelastic systems.

In the third chapter of this thesis I will simulate dynamos under the anelastic approximation and analyse the effect of incorporating density stratification in spherical shell 
dynamo on the resulting scaling behaviour of magnetic field and other interesting quantities. The focus will be on analysing a very diverse set of numerical simulations to check the validity of scaling laws. I will discuss how the parameter $R a_{Q}^{*}$ is not a good parameter for scaling laws in compressible dynamos and how averaging quantities appropriately has significant importance. With this exercise I will show that as far the mean properties are concerned both Boussinesq and compressible dynamos in rapidly-rotating shells are very similar.

\subsubsection{Focus of Chapter 4}

It has long been known that sunspot travel at different speeds at different latitudes on the surface of the Sun. This indicated that the plasma on the solar surface is rotating differentially, with equator being faster than poles. This behaviour has been called "differential rotation". Tracking the features on the surface of the giant planets has established that their surface also rotates differentially. Intriguingly, in planets, there are two different regimes. Jupiter and Saturn both have faster velocities in equatorial regions, hence, both have a solar-like differential rotation. On the other hand, Uranus and Neptune have slower equatorial velocities, therefore, their differential rotation is anti-solar in nature.

Remarkably, differential rotation appears to be a fundamental feature of rotating convection. In the past, differential rotation has been studied by both planetary and stellar convection communities. Unfortunately, although many things are common in the simulations of both of these communities (including the control parameters), there has not been any coherent analysis of the reported results. In chapter 4 of this thesis we try to fill this gap. We analyse a combined data-set consisting of new simulations (Boussinesq and anlastic) as well simulations from earlier studies of planetary and stellar convection zones (Boussinesq, anelastic, and fully-compressible). With this exercise we aim to provide a common framework of differential rotation which is applicable to both planets and stars. Generally, Coriolis force tries to promote 2 dimensionality and favours the formation of axially-aligned columnar convection, while buoyancy forces try to introduce isotropy in the convection and favour Rayleigh-Bénard type convection. We discuss in that chapter that the nature of the differential rotation near the equator is solar-like when Coriolis forces dominate and it changes to anti-solar behaviour when buoyancy forces dominate. This transition can be well-captured by a parameter which roughly quantifies the ratio of Coriolis and inertial (or buoyancy) forces. We also discuss a rather interesting behaviour where the differential rotation in a simulation depends on the initial condition of the simulation. The effect of magnetic on the differential rotation is also discussed.

\subsubsection{Focus of Chapter 5}

In the last chapter of this thesis I digress from the approach taken so far in previous chapters. Instead of carrying out a parameter study based on performing simulations with relatively moderate computational requirements to study the behaviour of mean properties of the dynamos, I will present a handful of state-of-the-art high resolution simulations to shed light on some unique features seen in some stars.

Helioseismology has revealed that there is a region of strong radial shear, called the "tachocline", about 30\% below the photosphere of the Sun (Schou et al. 1998). This 
region defines a rough boundary between the almost solid-body rotation of the radiative interior and the differentially rotating convection zone. It is safe to assume that this tachocline region will also be present in other non-fully convective stars as well. Due to the large shear the tachocline is especially potent at converting poloidal magnetic field lines to toroidal magnetic field lines. In the Sun (probably in other Sun-like stars as well) this tachocline is a geometrically large entity, and the solar latitudinal differential rotation is also large. It is thus not very surprising that the solar dynamo has been categorized as an $\alpha \Omega$-dynamo (Charbonneau 2005). It is believed that the helical small-scale turbulence provides the $\alpha$ effect while the large-scale shear provides the $\Omega$ effect in the Sun.

On the other hand, stars which rotate faster and have smaller radiative cores might be like Earth and Jupiter in many respects. First, since the radiative cores are small, the tachocline region in such stars will be geometrically tiny. Therefore, the plausible $\Omega$-effect might be rather small. Secondly, the convection in such stars will be rotationally dominated, and parameter studies performed in the planetary dynamo community have shown that such type of convection is very efficient at generating dynamo with dipole dominated magnetic fields (e.g. Jones et al. 2011). Olson et al. (1999) have classified such rotationally dominated dynamos as $\alpha^{2}$-type, i.e. the small scale helical flow itself generates both toroidal and poloidal magnetic field. These dynamos usually produce magnetic fields whose energy content equals or exceeds that of the kinetic energy of the flow. Such strong dipolar magnetic field actually suppresses the development of a strong latitudinal shear. Consequently, such dynamos are accompanied by negligible differential rotation. In this context, the fact that there is no observational evidence for strong differential rotation in the Earth, Jupiter and Saturn likely have very small differential rotation in the deeper dynamo region (Liu et al. 2008, Gastine et al. 2014), and rapidly-rotating stars with a dipole dominant magnetic field also show almost negligible differential rotation (Donati and Landstreet 2009), is supportive of this picture of $\alpha^{2}$-type dynamos. These intriguing evidences demand at least an exploratory investigation of the stellar dynamos using a simulation setup where density stratification is high, rotational effects are strong, and a tachocline is non-existent.

Let us now elaborate a little on the "specific features" which we alluded to in the beginning of this subsection. Appearance of dark spots on the surface of our Sun is a common phenomenon. On the Sun, the strongest magnetic field is found in these spots. The most popular explanation for the sunspot formation resides on the idea that convection is quenched when magnetic field is applied to it. It is believed that thin tubes of strong toroidal magnetic flux in the solar tachocline become buoyantly unstable after the field strength reaches a very high value. These flux-tubes rise through the convection zone, and when they encounters the granular convection in the outermost convective layer (the photosphere) they severely inhibit the convection locally. Since convection is transporting heat, such inhibition acts like a local "plug" and stops a lot of heat from coming out. As a result, the plasma becomes cooler as compared to the other regions without strong magnetic fields and it appears darker.

As the Sun goes through its activity cycles the number of sunspots and the latitude at which they appear varies. However, the sunspots mostly stay within a low-latitude belt ranging from $30^{\circ}$ north and south of the solar equator. It has almost been 3 decades now since Vogt et al. (1987) introduced a technique called "Doppler imaging" which allows 
one to map the surface brightness variations of a rapidly-rotating $\operatorname{star}^{8}$. As of now, this technique has shown the existence of dark spot on numerous stars. In fact, we have been able to infer some statistical trends in the data-set comprising of stars with different rotation speeds and spectral classes (Berdyugina 2005, Strassmeier 2009). Although it is not firmly established yet, there are indications that rapidly-rotating stars tend to harbour spots at high latitudes. Big dark regions ${ }^{9}$, much bigger than the largest recorded active regions on the Sun, have been observed in many such stars. In this context, the fact that Earth and Jupiter like dipole-dominated magnetic field in other stars would be stronger at high-latitudes and would preferably generate spots in polar regions is particularly noteworthy.

There have been several attempts in the past to explain why rapidly-rotating stars preferably generate dark spots at high latitudes. One of the most studied approach is basically an extension of the flux-tube based solar dynamo models mentioned above. Schüssler and Solanki (1992) conjectured that if the solar-like flux-tube scenario also applies to other rapidly-rotating stars, then the increased Coriolis forces will deflect the rising flux tubes to higher latitudes, essentially making them follow a cylindrically upward path. In contrast, since Sun rotates slowly, the associated Coriolis force is weaker than the radially directed buoyancy force, and it does not alter the path of the rising flux-tubes. One obvious problem with such an approach is that it heavily relies on the existence of a tachocline. Therefore stars where radiative cores are not present ${ }^{10}$ this model is clearly not applicable.

Another important aspect still missing in the spot-formation studies is self-consistency. There are basically two communities in this context: on one hand there are researchers who study the dynamo properties of the Sun and other stars on a global scale through different modelling techniques and presume that the resulting magnetic field will lead to dark spot formation, and on the other hand we have modellers who presume the existence of magnetic field and study the formation mechanism of dark spots in a local Cartesian box geometry. Of course, in reality, these things are coupled and should be modelled together. So far, this has not been achieved.

Keeping in mind the intriguing similarities between the magnetic field of the Earth and that of the rapidly-rotating stars, and the drawbacks of conventional solar models mentioned above, I set out to explore what would the wisdom gained through the numerical studies of planetary dynamos produce if applied to the realm of stellar dynamos. Chapter 5 is dedicated to this approach where I use high resolution numerical simulations to study dynamos in rapidly-rotating spherical shells with a density-stratified convection zone. With these models I demonstrate that a simulation which is sufficiently rapidly-rotating and has high-enough density stratification can spontaneously generate dark spots. These

\footnotetext{
${ }^{8}$ The coming-closer and going-away parts of the visible stellar disk produce blue and red shifted spectral lines which carry information about the corresponding regions. When an observer records one spectral line for a certain point-like star the red/blue shifted spectral lines define the different parts of the final spectral line shape. If we record spectral line profiles as a function of time (as the star rotates) the unique surface features will move from red(blue)-shifted part to blue(red)-shifted part in the recorded spectral line. Using different techniques one can now back-infer the stellar surface features from the line profiles. However, due to its vary nature, solution is not unique and regularization procedures (for instance, entropy minimization) are mandatory.

${ }^{9}$ Most likely they are big active-regions (much like the Sun) rather than individual big spots.

${ }^{10}$ Young $\mathrm{T}$ Tauri stars and low-mass stars with mass below $1 / 3 \mathrm{rd}$ of the mass of the Sun.
} 
dark spots self-consistently appear at high-latitudes. These results provide an alternative explanation to the puzzle of high-latitude starspots in rapidly-rotating stars. 



\section{Bibliography}

Aubert, J. (2005). Steady zonal flows in spherical shell dynamos. J. Flu. Mech., 542(1), 53-67.

Aubert, J., Brito, D., Nataf, H.-C., Cardin, P., and Masson, J.-P. (2001). A systematic experimental study of rapidly rotating spherical convection in water and liquid gallium. Phys. Earth Planet. Int., 128(1-4), 51-74.

Backus, G. (1958). A class of self-sustaining dissipative spherical dynamos. Annals Phys., 4(4), 372-447.

Batchelor, G. K. (1953). The conditions for dynamical similarity of motions of a frictionless perfect-gas atmosphere. Quart. J. Royal Meteor. Soc., 79(340), 224-235.

Berdyugina, S. V. (2005). Starspots: A key to the stellar dynamo. Liv. Rev. Sol. Phys., 2(8).

Blackett, P. (1947). The magnetic field of massive rotating bodies. Nature, 159, 658-666.

Braginsky, S. I. and Roberts, P. H. (1995). Equations governing convection in earth's core and the geodynamo. Geophys. E Astrophys. Flu. Dyn., 79(1-4), 1-97.

Brandenburg, A. and Subramanian, K. (2005). Astrophysical magnetic fields and nonlinear dynamo theory. Phys. Rep., 417(1), 1-209.

Busse, F. (1975). A model of the geodynamo. Geophys. J. Int., 42(2), 437-459.

Busse, F. (1976). Generation of planetary magnetism by convection. Phys. Earth Plan. Inte., 12(4), 350-358.

Cain, J. C., Beaumont, P., Holter, W., Wang, Z., and Nevanlinna, H. (1995). The magnetic bode fallacy. J. Geophys. Res.: Planets, 100(E5), 9439-9454.

Chapman, S. and Bartels, J. (1940). Geomagnetism. Oxford: Claredon Press.

Charbonneau, P. (2005). Dynamo models of the solar cycle. Liv. Rev. Sol. Phys., 2(2).

Christensen, U., Olson, P., and Glatzmaier, G. (1999). Numerical modelling of the geodynamo: a systematic parameter study. Geophys. J. Int., 138(2), 393-409.

Christensen, U. R. (2002). Zonal flow driven by strongly supercritical convection in rotating spherical shells. J. Flu. Mech., 470, 115-133. 
Christensen, U. R. and Aubert, J. (2006). Scaling properties of convection-driven dynamos in rotating spherical shells and application to planetary magnetic fields. Geophys. J. Int., 166(1), 97-114.

Christensen, U. R. and Tilgner, A. (2004). Power requirement of the geodynamo from ohmic losses in numerical and laboratory dynamos. Nature, 429, 169-171.

Christensen, U. R., Holzwarth, V., and Reiners, A. (2009). Energy flux determines magnetic field strength of planets and stars. Nature, 457, 167.

Curtis, S. and Ness, N. (1986). Magnetostrophic balance in planetary dynamos: Predictions for neptune's magnetosphere. J. Geophys. Res.: Spa. Phys., 91(A10), 1100311008 .

DeRosa, M. L. (2001). Dynamics in the upper solar convection zone. Ph.D. thesis, University of Colorado.

Donati, J.-F. and Landstreet, J. D. (2009). Magnetic fields of nondegenerate stars. Annu. Rev. Astron. Astrophys., 47, 333-370.

Gastine, T., Wicht, J., Duarte, L., Heimpel, M., and Becker, A. (2014). Explaining jupiter's magnetic field and equatorial jet dynamics. Geophys. Res. Lett., 41(15), 54105419.

Gilman, P. A. (1977). Nonlinear dynamics of boussinesq convection in a deep rotating spherical shell-i. Geophys. E Astrophys. Flu. Dyn., 8(1), 93-135.

Gilman, P. A. and Glatzmaier, G. A. (1981). Compressible convection in a rotating spherical shell. i-anelastic equations. ApJS, 45, 335.

Glatzmaier, G. A. and Roberts, P. H. (1995a). A three-dimensional convective dynamo solution with rotating and finitely conducting inner core and mantle. Phys. Earth and Plan. Inte., 91(1), 63-75.

Glatzmaier, G. A. and Roberts, P. H. (1995b). A three-dimensional self-consistent computer simulation of a geomagnetic field reversal. Nature, 377, 203-209.

Glatzmaier, G. A., Evonuk, M., and Rogers, T. M. (2009). Differential rotation in giant planets maintained by density-stratified turbulent convection. Geophys. $\mathcal{F}$ Astrophys. Flu. Dyn., 103(1), 31-51.

Jones, C., Boronski, P., Brun, A., Glatzmaier, G., Gastine, T., Miesch, M., and Wicht, J. (2011). Anelastic convection-driven dynamo benchmarks. Icarus, 216(1), 120-135.

Jones, C. A. (2011). Planetary magnetic fields and fluid dynamos. Ann. Rev. Flu. Mech., 43(1), 583-614.

Kageyama, A. and Sato, T. (1995). Computer simulation of a magnetohydrodynamic dynamo. ii. Phys. Plas., 2(5), 1421-1431. 
King, E. M. and Buffett, B. A. (2013). Flow speeds and length scales in geodynamo models: The role of viscosity. Earth and Planetary Science Letters, 371, 156-162.

Krause, F. and Rädler, K.-H. (1980). Mean-field magnetohydrodynamics and dynamo theory. Pergamon Press Oxford.

Lantz, S. and Fan, Y. (1999). Anelastic magnetohydrodynamic equations for modeling solar and stellar convection zones. ApJS, 121(1), 247-264.

Liu, J., Goldreich, P. M., and Stevenson, D. J. (2008). Constraints on deep-seated zonal winds inside jupiter and saturn. Icarus, 196(2), 653-664.

Mizutani, H., Yamamoto, T., and Fujimura, A. (1992). A new scaling law of the planetary magnetic fields. Advan. Spa. Res., 12(8), 265-279.

Moffatt, H. K. (1978). Magnetic Field Generation in Electrically Conducting Fluids. Cambridge University Press, Cambridge, London, New York, Melbourne.

Müller, U. and Stieglitz, R. (1999). The karlsruhe dynamo experiment. Non. Proc. in Geophys., 9(3/4), 165-170.

Ogura, Y. and Phillips, N. A. (1962). Scale analysis of deep and shallow convection in the atmosphere. J. Atmos. sci., 19(2), 173-179.

Olson, P., Christensen, U., and Glatzmaier, G. A. (1999). Numerical modeling of the geodynamo: mechanisms of field generation and equilibration. J. Geophys. Res., 104(B5), 10383-10404.

Pizzolato, N., Maggio, A., Micela, G., Sciortino, S., and Ventura, P. (2003). The stellar activity-rotation relationship revisited: Dependence of saturated and non-saturated $\mathrm{x}$ ray emission regimes on stellar mass for late-type dwarfs. $A \mathcal{E} A$, 397(1), 147-158.

Reiners, A. (2012). Observations of cool-star magnetic fields. Liv. Rev. Sol. Phys., 9(1).

Reshetnyak, M. Y. (2012). Effect of compressibility on the generation of hydrodynamic helicity in the Earth's liquid core. Geomagnetism and Aeronomy, 52(3), 398-402.

Sano, Y. (1993). The magnetic fields of the planets: a new scaling law of the dipole moments of the planetary magnetism. J. geomag. geoelec., 45(1), 65-77.

Schou, J., Antia, H., Basu, S., Bogart, R., Bush, R., Chitre, S., Christensen-Dalsgaard, J., Di Mauro, M., Dziembowski, W., Eff-Darwich, A., et al. (1998). Helioseismic studies of differential rotation in the solar envelope by the solar oscillations investigation using the michelson doppler imager. ApJ, 505(1), 390.

Schüssler, M. and Solanki, S. (1992). Why rapid rotators have polar spots. $A \mathcal{E} A, \mathbf{2 6 4}$, L13-L16.

Semel, M. (1989). Zeeman-Doppler imaging of active stars. I - Basic principles. AEEA, 225, 456-466. 
Soderlund, K. M., King, E. M., and Aurnou, J. M. (2012). The influence of magnetic fields in planetary dynamo models. Earth Plan. Sci. Let., 333, 9-20.

Solanki, S. K., Inhester, B., and SchÃijssler, M. (2006). The solar magnetic field. Rep. Prog. Phys., 69(3), 563.

Starchenko, S. and Jones, C. (2002). Typical velocities and magnetic field strengths in planetary interiors. Icarus, 157(2), 426-435.

Stevenson, D. (1983). Planetary magnetic fields. Rep. Prog. Phys., 46(5), 555.

Stevenson, D. J. (1979). Turbulent thermal convection in the presence of rotation and a magnetic field: a heuristic theory. Geophys. $\mathcal{E}$ Astrophys. Flu. Dyn., 12(1), 139-169.

Strassmeier, K. G. (2009). Starspots. AEAA Rev., 17(3), 251-308.

Vogt, S. S., Penrod, G. D., and Hatzes, A. P. (1987). Doppler images of rotating stars using maximum entropy image reconstruction. ApJ, 321, 496-515. 


\title{
2 Scaling laws in spherical shell dynamos with free-slip boundaries
}

\begin{abstract}
Numerical simulations of convection driven rotating spherical shell dynamos have often been performed with rigid boundary conditions, as is appropriate for the metallic cores of terrestrial planets. Free-slip boundaries are more appropriate for dynamos in other astrophysical objects, such as gas-giants or stars. Using a set of 57 direct numerical simulations, we investigate the effect of free-slip boundary conditions on the scaling properties of heat flow, flow velocity and magnetic field strength and compare it with earlier results for rigid boundaries. We find that the nature of the mechanical boundary condition has only a minor influence on the scaling laws. We also find that although dipolar and multipolar dynamos exhibit approximately the same scaling exponents, there is an offset in the scaling pre-factors for velocity and magnetic field strength. We argue that the offset can be attributed to the differences in the zonal flow contribution between dipolar and multipolar dynamos ${ }^{1,2}$.
\end{abstract}

\subsection{Introduction}

Numerical simulations of dynamos in geometries appropriate for the cores of terrestrial planets have greatly enhanced our understanding of the complex magnetic field behavior observed in these objects, with possible implications for a broader class of dynamos in astrophysical objects (Jones 2011). One of the major drawbacks of such simulations is that there is order of magnitude disagreement between the natural and the numerically accessible values of several control parameters. For instance, in numerical simulations of the geodynamo, the Ekman number - a nondimensional measure of the importance of viscous effects as compared to the Coriolis effects - is usually five to ten orders of magnitude larger than the expected realistic values.

One way to tackle this disparity is to infer asymptotic scaling laws from a sufficient number of numerical results. Such numerical scaling laws can then be extrapolated to realistic parameter regimes and compared with the observational data. Christensen and Aubert (2006) (hereafter "CA6") used a battery of numerical simulation results to derive scaling relations for heat transfer, convective velocity, and magnetic field strength.

\footnotetext{
${ }^{1}$ The content of this chapter has been reproduced from: R. K. Yadav, T. Gastine, and U. R. Christensen, "Scaling laws in spherical shell dynamos with free-slip boundaries", Icarus 225, 185, 2013. DOI: j.icarus.2013.02.030

${ }^{2}$ Contribution: I ran the simulations used in the study and carried out the analysis. I wrote the first draft of the manuscript; despite changes in later revisions the original structure of the manuscript was preserved.
} 
Their scaling relations hold over several orders of magnitude of the relevant control parameter. Using these scaling relations, CA6 predicted magnetic field strengths inside the Earth's and Jupiter's core and found reasonable agreement with observationally constrained values. Later, Christensen et al. (2009) showed that these scaling laws are also in good agreement with magnetic fields observed in fast rotating low-mass stars. Takahashi et al. (2008) and Aubert et al. (2009) independently reinforced the scaling laws put forth by CA6. Olson and Christensen (2006) derived scaling laws specifically for the dipole moment of planetary dynamos which show an order-of-magnitude agreement with the observed dipole moments of solar system planets. Christensen (2010) reviews earlier scaling laws for planetary magnetic field based on heuristic arguments and compares them with the numerically established scaling relations.

The mechanical boundary conditions may play an important role in the dynamo mechanism. Dynamos which operate in planets with a solid mantle are usually modeled with rigid boundaries (Kageyama and Sato 1995, Glatzmaier and Roberts 1995a,b). While a true free surface is difficult to model, a free-slip condition (i.e. assuming zero shear stress at an undeformable spherical boundary) is a much better approximation than a no-slip condition for the surface of gas- and ice-giant planets or stellar convection zones. Rigid boundaries are associated with viscous (Ekman) boundary layers, which have a damping influence on the development of strong axisymmetric flows that are found in free surface flows. Kuang and Bloxham $(1997,1999)$ argued that even for the geodynamo a freeslip condition may be a better choice, because the Ekman layers in the models are much thicker than the very thin layers in the Earth's core. Aubert et al. (2001) demonstrated in rotating liquid Gallium experiments that at small Prandtl numbers - the ratio of kinematic viscosity to thermal diffusivity - flows with rigid boundaries show features similar to those with free-slip boundaries, i.e. dominant zonal flows. Miyagoshi et al. (2010) also found strong zonal flows in low Ekman number $\left(\approx 10^{-7}\right)$ rigid boundary geodynamo simulations. Hence, studies of dynamos with free-slip boundaries have wide ranging applications. Following these arguments, researchers have modelled Uranus' and Neptune's multipolar magnetic field (Stanley and Bloxham 2004, 2006), Mercury's weak magnetic field (Stanley et al. 2005), and Saturn's unusually axisymmetric magnetic field (Stanley 2010) using free-slip boundaries.

Dynamos with free-slip boundaries also exhibit bistability: the morphology of the dynamo generated magnetic field depends on the initial magnetic field configuration (Simitev and Busse 2009, 2012, Sasaki et al. 2011, Schrinner et al. 2012, Gastine et al. 2012). Recently, Gastine et al. (2013) found evidence of bistability in M dwarfs. Grote and co-workers (Grote et al. 1999, Grote and Busse 2000, Grote et al. 2000) have employed free-slip boundaries in their dynamo models, and found a wider spectrum of magnetic field geometries than what has been reported for rigid boundaries.

A direct comparison of the effects of different mechanical boundary conditions on the dynamo has rarely been made. Christensen et al. (1999) reported results for both kinds of boundary conditions for a limited number of cases and found that the large scale magnetic field is similar for both cases. Recently, Schrinner et al. (2012) have analyzed many dynamo simulations with rigid, free-slip and mixed (rigid at inner and free-slip at outer boundary) boundary conditions and report a difference in magnetic field amplitude of dipolar and multipolar dynamos. Following this study, we specifically focus here on deriving scaling properties for heat transport, velocity, and magnetic field strength. We 
compare our findings with earlier rigid boundary systems for which extensive modeling results are available in a broad range of control parameters (CA6). This exercise helps us in isolating the effect of mechanical boundary condition.

\subsection{Dynamo model}

\subsubsection{MHD equations}

Our numerical set-up consists of a spherical shell which rotates along the $\hat{z}$-axis and which is bounded by inner radius $r_{i}$ and outer radius $r_{o}$. The aspect ratio $r_{i} / r_{o}$ is 0.35 . A linear variation of gravity with radius is assumed. Following CA6, we non-dimensionalize the magnetohydrodynamic (MHD) equations by using the shell thickness $r_{o}-r_{i}=D$ as the reference length scale and $1 / \Omega$, where $\Omega$ is the rotation rate, as the time unit. The magnetic field $\mathbf{B}$ is scaled by $\Omega D \sqrt{\rho \mu}$, where $\rho$ is the constant fluid density and $\mu$ is the magnetic permeability. Note that all of the above non-dimensional scales are free from any diffusion parameters. The temporal evolution of velocity $\mathbf{u}$, temperature $T$, and magnetic field $\mathbf{B}$ is governed by the MHD equations under the Boussinesq approximation

$$
\begin{aligned}
\frac{\partial \mathbf{u}}{\partial t}+\mathbf{u} \cdot \nabla \mathbf{u}+2 \hat{z} \times \mathbf{u}+\nabla P & =R a^{*} \frac{\mathbf{r} T}{r_{o}}+(\nabla \times \mathbf{B}) \times \mathbf{B}+E \nabla^{2} \mathbf{u}, \\
\frac{\partial T}{\partial t}+\mathbf{u} \cdot \nabla T & =\frac{E}{P r} \nabla^{2} T, \\
\frac{\partial \mathbf{B}}{\partial t}=\nabla \times(\mathbf{u} \times \mathbf{B})+\frac{E}{P m} \nabla^{2} \mathbf{B}, & \\
\nabla \cdot \mathbf{u} & =0, \\
\nabla \cdot \mathbf{B} & =0 .
\end{aligned}
$$

This system of equations is governed by several nondimensional control-parameters: Ekman number $E=v / \Omega d^{2}, v$ being the fluid viscosity; the modified Rayleigh number $R a^{*}=\alpha g_{o} \Delta T / \Omega^{2} D$, where $g_{o}$ is gravity at the outer boundary and $\alpha$ is the thermal expansivity; magnetic Prandtl number $P m=v / \eta, \eta$ being the magnetic diffusivity; Prandtl number $\operatorname{Pr}=v / \kappa, \kappa$ being the thermal conductivity. $R a^{*}$ is related to the conventional Rayleigh number $R a$ through $R a^{*}=R a E^{2} / P r$.

We assume free-slip mechanical boundaries at both inner and outer radius. The magnetic field matches a potential field at both boundaries. A fixed temperature contrast $\Delta T$ is maintained between the top and the bottom.

\subsubsection{Numerical method}

Equations (2.1-2.5) are numerically solved using the MagIC code (Wicht 2002). Velocity and magnetic field are first separated into toroidal and poloidal components as

$$
\begin{aligned}
\mathbf{u} & =\nabla \times u_{T} \hat{r}+\nabla \times \nabla \times u_{P} \hat{r}, \\
\mathbf{B} & =\nabla \times B_{T} \hat{r}+\nabla \times \nabla \times B_{P} \hat{r} .
\end{aligned}
$$


The scalar potentials $u_{T, P}$ and $B_{T, P}$, along with temperature $T$ and pressure $P$, are further expanded using spherical harmonics in the $\theta$ and $\phi$ directions and the Chebyshev polynomials in the radial direction. $N_{r}$ and $l_{\max }$ are the maximum degree of the Chebyshev polynomials and the spherical harmonic functions used in this expansion. For all the simulations considered here, $41 \leq N_{r} \leq 73$ and $64 \leq l_{\max } \leq 170$. The simulations are run for at least one magnetic diffusion time $\left(D^{2} / \eta\right)$ to ensure a statistically stationary state.

\subsubsection{Diagnostic parameters}

We employ several diagnostic parameters to analyze our simulations results. The Rossby number $R o$ is the volume averaged non-dimensional rms velocity. Following CA6, we also introduce the local Rossby number $R o_{l}$ which is a more appropriate measure than $R o$ to characterize the ratio of the inertial and the Coriolis forces. A typical flow length scale can be calculated based on the mean spherical harmonic degree $l$

$$
\bar{l}_{u}=\sum_{l} l \frac{\left\langle\mathbf{u}_{l} \cdot \mathbf{u}_{l}\right\rangle}{\langle\mathbf{u} \cdot \mathbf{u}\rangle},
$$

where $\langle\ldots\rangle$ denotes time average and $\mathbf{u}_{l}$ is velocity component at degree $l$. The local Rossby number is then defined as $R o_{l}=R o \pi / \bar{l}_{u}$.

The volume averaged non-dimensional rms magnetic field strength is called Lorentz number Lo. The field geometry at the outer boundary surface is characterized by its dipolarity $f_{\text {dip }}$. It is defined as the ratio of the magnetic energy of the dipole to the total magnetic energy at the outer boundary surface.

The Nusselt number $N u$ is a ratio of total heat transported from the inner shell to the outer shell to the conducted heat. It is expected that different diffusivities play a minimal role in determining the large scale properties of the dynamo systems. This motivated CA6 to define a modified Nusselt number $N u^{*}$ which does not involve $\kappa . N u^{*}$ is related to the conventional Nusselt number $N u$ via $N u^{*}=(N u-1) E / P r$. In addition, the heat flux from surfaces of astrophysical objects is a much more meaningful and accessible quantity than the temperature difference between the inner and outer boundary of the convection zone. CA6 defined a heat flux based Rayleigh number $R a_{Q}^{*}$ which incorporates the advected heat flux rather than the temperature contrast. $R a_{Q}^{*}$ is related to $R a$ through $R a_{Q}^{*}=R a(N u-1) E^{3} / \operatorname{Pr}^{2}$.

The reported numerical values of the diagnostic parameters $R o, R o_{l}, L o, N u^{*}$, and $f_{d i p}$ are time averaged values excluding the initial transients.

\subsection{Results}

We have built up a data set of 57 dynamo simulations with free-slip boundaries: 40 cases by us (see Table 2.1) and 17 cases adopted from Schrinner et al. (2012). All of the cases in this data set have the same physical set-up as described above. Also, following CA6, we report and analyze only those dynamo simulations which have $N u>2$ to ensure a vigorous enough convection that fills the full volume of the spherical shell. 
Table 2.1: Results for Prandtl number $P r=1$ dynamo simulations. The $P m$ values of bistable multipolar dynamos are marked with "*". The critical Rayleigh numbers $R a_{c}$ at which fluid convection is first excited are: $4.99 \times 10^{4}\left(E=1 \times 10^{-3}\right), 1.86 \times 10^{5}$ $\left(E=3 \times 10^{-4}\right), 6.51 \times 10^{5}\left(E=1 \times 10^{-4}\right), 2.68 \times 10^{6}\left(E=3 \times 10^{-5}\right), 1.03 \times 10^{7}$ $\left(E=1 \times 10^{-5}\right)$.

\begin{tabular}{|c|c|c|c|c|c|c|c|c|c|}
\hline$E$ & $P m$ & $R a / R a_{c}$ & $N u$ & $R m$ & $R o_{l}$ & $R o_{\text {zonal }}$ & Lo & $f_{\text {dip }}$ & $f_{\text {ohm }}$ \\
\hline \multirow[t]{7}{*}{$10^{-3}$} & 5 & 10.02 & 2.42 & 322.14 & 0.116 & 0.0477 & 0.0210 & 0.0078 & 0.15 \\
\hline & 5 & 20.04 & 4.12 & 515.39 & 0.253 & 0.0540 & 0.0454 & 0.0050 & 0.21 \\
\hline & 5 & 30.06 & 5.21 & 701.42 & 0.353 & 0.0540 & 0.0603 & 0.0030 & 0.24 \\
\hline & 5 & 40.08 & 6.68 & 1223.29 & 0.375 & 0.0966 & 0.0744 & 0.0120 & 0.26 \\
\hline & 5 & 50.10 & 7.23 & 1412.75 & 0.411 & 0.1253 & 0.0819 & 0.0055 & 0.25 \\
\hline & 2 & 40.08 & 7.03 & 635.74 & 0.388 & 0.1866 & 0.0190 & 0.0070 & 0.03 \\
\hline & 2 & 50.10 & 7.57 & 685.70 & 0.442 & 0.1971 & 0.0329 & 0.0064 & 0.07 \\
\hline \multirow[t]{18}{*}{$3 \times 10^{-4}$} & 10 & 6.99 & 2.18 & 376.64 & 0.038 & 0.0033 & 0.0302 & 0.4193 & 0.61 \\
\hline & 5 & 8.06 & 2.27 & 230.73 & 0.048 & 0.0041 & 0.0253 & 0.6320 & 0.57 \\
\hline & $5^{*}$ & 8.06 & 2.14 & 297.35 & 0.057 & 0.0099 & 0.0115 & 0.0151 & 0.25 \\
\hline & 5 & 10.75 & 2.86 & 324.21 & 0.080 & 0.0041 & 0.0265 & 0.4800 & 0.48 \\
\hline & 5 & 13.44 & 3.41 & 489.15 & 0.108 & 0.0138 & 0.0203 & 0.0036 & 0.31 \\
\hline & 5 & 16.13 & 3.94 & 562.90 & 0.128 & 0.0151 & 0.0238 & 0.0050 & 0.34 \\
\hline & 3 & 8.06 & 2.20 & 142.04 & 0.051 & 0.0037 & 0.0212 & 0.7620 & 0.50 \\
\hline & $3 *$ & 8.06 & 2.09 & 185.08 & 0.053 & 0.0117 & 0.0103 & 0.0121 & 0.23 \\
\hline & 3 & 11.29 & 2.86 & 270.97 & 0.080 & 0.0167 & 0.0156 & 0.0130 & 0.28 \\
\hline & 3 & 14.52 & 3.57 & 335.20 & 0.108 & 0.0206 & 0.0197 & 0.0127 & 0.31 \\
\hline & 1.5 & 8.60 & 2.02 & 110.82 & 0.046 & 0.0177 & 0.0086 & 0.0180 & 0.19 \\
\hline & 1.5 & 9.14 & 2.20 & 116.79 & 0.053 & 0.0179 & 0.0101 & 0.0240 & 0.21 \\
\hline & 1.5 & 9.68 & 2.37 & 121.74 & 0.060 & 0.0180 & 0.0114 & 0.0210 & 0.23 \\
\hline & 1.5 & 10.22 & 2.52 & 129.47 & 0.065 & 0.0189 & 0.0123 & 0.0270 & 0.24 \\
\hline & 1.5 & 10.75 & 2.60 & 138.66 & 0.067 & 0.0206 & 0.0128 & 0.0120 & 0.25 \\
\hline & 1 & 10.75 & 2.32 & 103.27 & 0.054 & 0.0265 & 0.0099 & 0.0068 & 0.20 \\
\hline & 1 & 13.44 & 3.12 & 119.57 & 0.082 & 0.0277 & 0.0155 & 0.0173 & 0.27 \\
\hline & 0.5 & 18.82 & 3.99 & 89.35 & 0.106 & 0.0451 & 0.0178 & 0.0188 & 0.24 \\
\hline \multirow[t]{10}{*}{$10^{-4}$} & 1 & 7.68 & 2.09 & 62.39 & 0.034 & 0.0013 & 0.0079 & 0.8741 & 0.42 \\
\hline & 1 & 8.45 & 2.26 & 69.76 & 0.038 & 0.0015 & 0.0087 & 0.8329 & 0.43 \\
\hline & 1 & 9.22 & 2.42 & 75.82 & 0.042 & 0.0017 & 0.0096 & 0.8004 & 0.43 \\
\hline & $1 *$ & 9.22 & 2.28 & 96.80 & 0.036 & 0.0062 & 0.0058 & 0.0086 & 0.29 \\
\hline & 1 & 12.29 & 2.99 & 98.48 & 0.058 & 0.0023 & 0.0123 & 0.7786 & 0.46 \\
\hline & $1^{*}$ & 12.29 & 3.11 & 130.97 & 0.057 & 0.0076 & 0.0079 & 0.0180 & 0.32 \\
\hline & 0.5 & 15.36 & 3.60 & 64.81 & 0.078 & 0.0028 & 0.0126 & 0.8891 & 0.41 \\
\hline & $0.5^{*}$ & 15.36 & 3.74 & 84.98 & 0.066 & 0.0111 & 0.0092 & 0.0115 & 0.34 \\
\hline & 0.5 & 30.72 & 5.91 & 109.98 & 0.131 & 0.0056 & 0.0210 & 0.7598 & 0.46 \\
\hline & 0.5 & 61.44 & 10.28 & 203.61 & 0.234 & 0.0137 & 0.0294 & 0.5634 & 0.43 \\
\hline \multirow[t]{4}{*}{$3 \times 10^{-5}$} & 1 & 9.33 & 2.41 & 104.84 & 0.025 & 0.0007 & 0.0046 & 0.6392 & 0.49 \\
\hline & 0.5 & 9.33 & 2.37 & 53.38 & 0.027 & 0.0006 & 0.0039 & 0.9071 & 0.39 \\
\hline & 0.5 & 11.19 & 2.81 & 65.25 & 0.035 & 0.0008 & 0.0047 & 0.8317 & 0.40 \\
\hline & $0.5 *$ & 11.19 & 2.78 & 80.30 & 0.032 & 0.0026 & 0.0030 & 0.0001 & 0.32 \\
\hline $10^{-5}$ & 0.2 & 14.56 & 3.44 & 49.68 & 0.032 & 0.0006 & 0.0023 & 0.9088 & 0.31 \\
\hline
\end{tabular}




\subsubsection{Bistability}

Bistability is a phenomenon in which a system shows two different dynamo solutions, i.e. dipolar and multipolar, for the same set of control parameters, but with different initial conditions for the magnetic field. For dynamos with free-slip boundaries, Simitev and Busse (2009) found two distinct dipolar and multipolar dynamo branches, at least in some parameter range. Sasaki et al. (2011), Schrinner et al. (2012), and Gastine et al. (2012) have also observed bistability in their dynamo simulations with free-slip boundaries. Dynamos in box geometry with periodic boundaries also show bistability (Yadav et al. 2012); in fact, even tristable and quadstable solutions were observed in such simulations. Bistable dynamo solutions have rarely been observed in dynamos with rigid boundaries, e.g. CA6 reported a single case in which they found bistable states. In dynamos with rigid boundaries, the dipolar branch collapses around $R o_{l} \approx 0.1$, which CA6 argue is due to inertial forces dominating over Coriolis force at larger $R o_{l}$, while Soderlund et al. (2012) argue that the dipole collapse is due to helicity degradation related to the competition of inertial and viscous forces. Soderlund et al. (2012) also hypothesize that current numerical planetary dynamo models are viscously controlled.

We plot in Fig. 2.1 the dipolarity $f_{d i p}$ at the outer surface $\left(r=r_{o}\right)$ versus the local Rossby number $R o_{l}$ (for our simulations). In the dipolar branch (filled data points) the magnetic field is dominated by the dipole $\left(f_{d i p}>0.4\right)$ and in the multipolar branch (empty data points) the dipole is much weaker $\left(f_{\text {dip }}<0.05\right)$. The dipole branch is limited to cases with $R o_{l} \lesssim 0.2$. However, the multipolar branch exists for a broad range of $R o_{l}$. The highly supercritical dipolar case at $R o_{l} \approx 0.2\left(E=1 \times 10^{-4}, P m=0.5\right.$ and $\left.R a=4 \times 10^{7}\right)$ was run for three magnetic diffusion times without an indication of a dipole collapse, although we can not exclude that the field could change to multipolar in the long run. Dipolar dynamos at such high $R o_{l}$ have not been reported yet. Simulations that settle down to different dynamo states depending on the initial magnetic field are marked with a "+" in Fig. 2.1 (difficult to discern on the multipolar branch due of clustering). Note that other dipolar dynamos could show bistability but we did not explore all of our dipolar cases for such behavior.

For rigid boundary dynamos, CA6 reported that multipolar dynamo solutions are not observed for $R o_{l}<0.1$. But results from Schrinner et al. (2012) and our findings suggest that this is not the case for free-slip boundaries. We also found a few dynamo solutions, which have $R o_{l}<0.1$, but settle to a multipolar solution despite having initial dipolar magnetic field. For example, we only found multipolar solutions for $E=3 \times 10^{-4}$ and $P m \leq 1.5$. This demonstrates that, depending on the control parameters, only the multipolar dynamo branch can be stable in some situations. This is in agreement with earlier results (Simitev and Busse 2009, 2012) which showed that bistability is in fact a function of $P, P m$ and $E$. They used volumetric heating while we use a fixed temperature contrast to drive convection. The bistable behavior we observed generalizes their findings.

One of the characteristic features of rotating spherical shell convection with free-slip boundaries is the development of strong axisymmetric zonal flows. In rigid boundary systems, the Reynolds stresses, which arise due to a statistical correlation between the radial and azimuthal flow component (in cylindrical co-ordinates), are balanced by the bulk viscosity and the Ekman layer friction near the outer boundary. In the case of free-slip boundaries, the Ekman layers are absent and zonal flows can thus saturate at much higher 


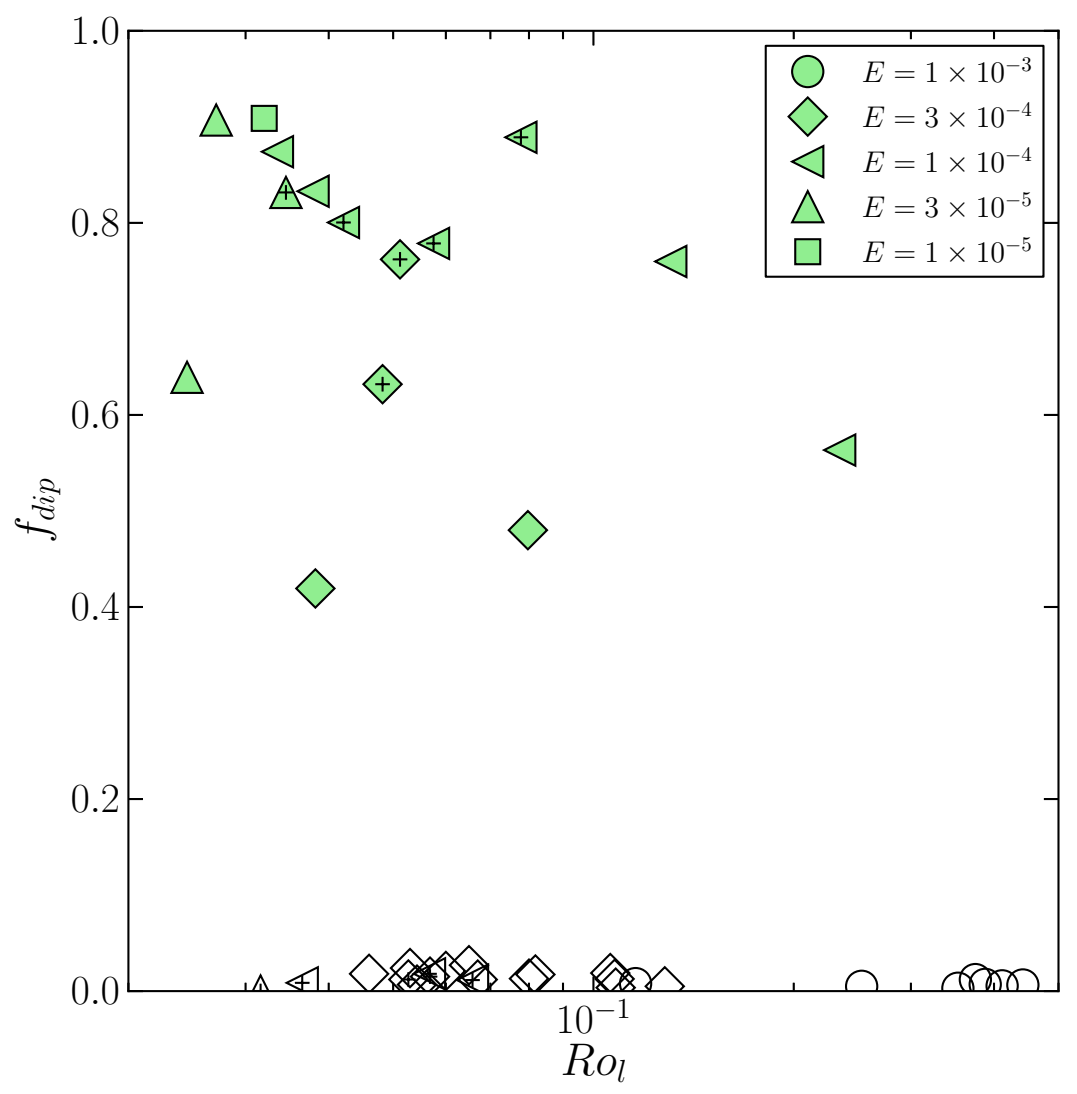

Figure 2.1: Dipolarity at the outer boundary versus the local Rossby number. The data points carrying "+" marker are bistable states. Filled (empty) symbols are dipolar (multipolar) dynamos. The symbol shapes represent the Ekman number and the corresponding value is given in the upper-right-corner box.

vigor. In dynamo models, Maxwell stresses also affect the zonal flows; these stresses are potentially higher in dipolar dynamos that have higher magnetic field strength than multipolar ones at the same control parameter values (Browning 2008). One argument for the essential role of zonal flows for bistability is that an initial dipolar magnetic field inhibits the growth of zonal flows via Maxwell stresses. In the case of a multipolar initial condition, strong zonal flows can develop, which in turn suppress the development of dipolar magnetic fields. This mechanism allows multipolar magnetic fields even for $R o_{l}$ smaller than 0.1 .

The zonal flow structure of a bistable state is shown in Fig. 2.2. It portrays a weak thermal wind driven zonal flow in the dipolar case and a nearly three times stronger and more geostrophic zonal flow in the multipolar case. Note that the magnetic field of the multipolar solution has a quadrupolar symmetry, but this is not generally the case. In particular in strongly driven cases the magnetic field has a smaller length scale and does not have any preferred symmetry. Aubert (2005) found that the thermal wind driven zonal flow topology is in agreement with Ferraro's law of co-rotation (Ferraro 1937), i.e. the 


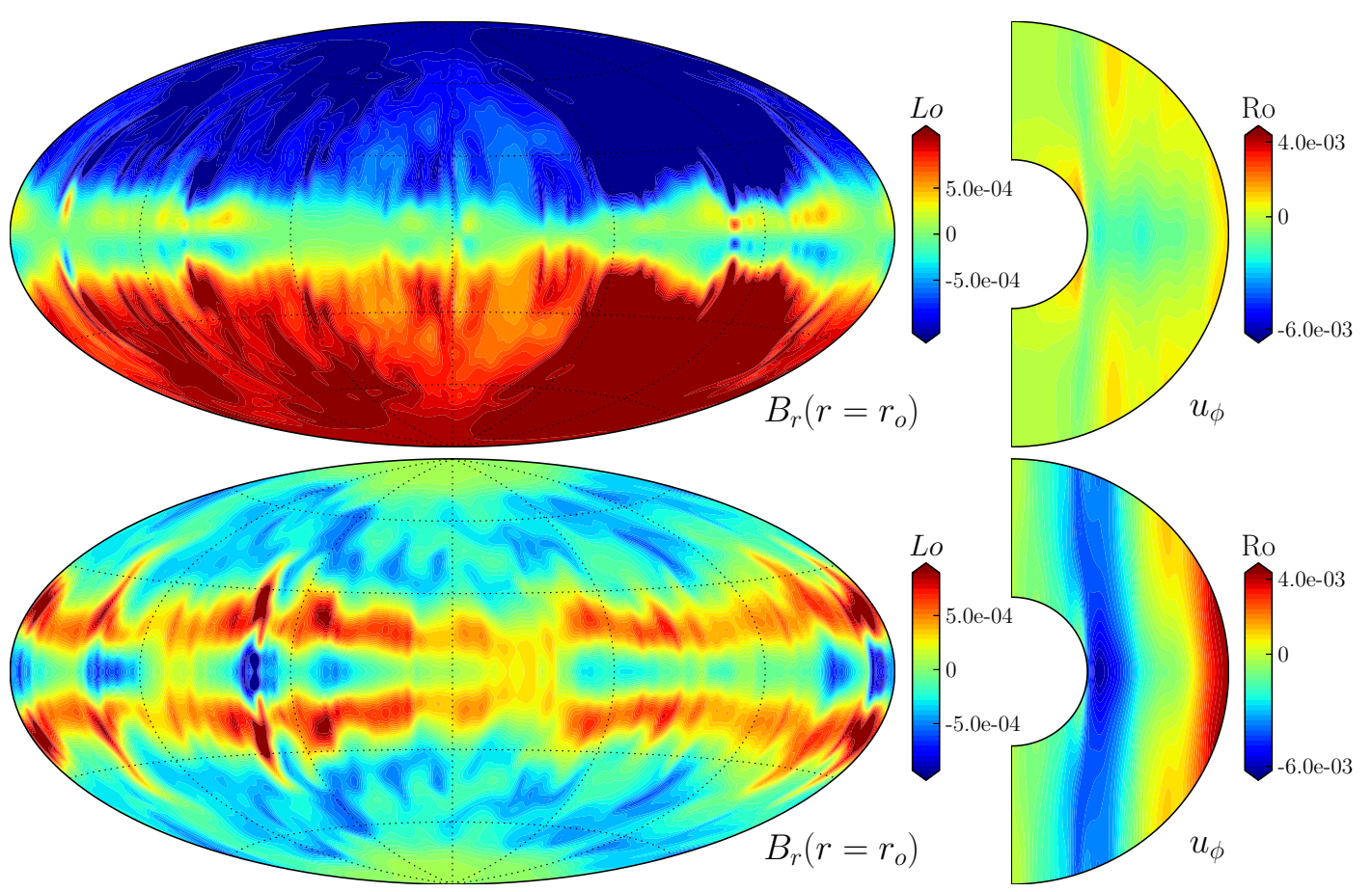

Figure 2.2: A snapshot of the non dimensional radial magnetic field at outer boundary and zonal flow (azimuthally averaged) in a meridional section for a dipolar (top row) and a multipolar (bottom row) dynamo. These states are obtained at $E=3 \times 10^{-5}, P m=0.5$, and $R a=3 \times 10^{7}$. The radial magnetic field is truncated at $50 \%$ of the maximum in order to highlight the magnetic field structures.

shearing of the axisymmetric poloidal magnetic field by the zonal flow is minimal. The non-geostrophy of the flow in case of the dipolar dynamo emphasizes that the zonal flow quenching by the Lorentz force is rather large in dipolar dynamos as compared to that in the multipolar dynamo cases, as also pointed out before by Schrinner et al. (2012).

\subsubsection{Nusselt number scaling}

Figure 2.3 shows that the modified Nusselt number $N u^{*}$ scales very well with the fluxbased Rayleigh number $R a_{Q}^{*}$ in the same way irrespective of whether the dynamo is dipolar (filled symbols) or multipolar (empty symbols). A best-fit line to this data set reveals a relation $N u^{*}=0.061 R a_{Q}^{* 0.52}$, which agrees well with the scaling $N u^{*}=0.076 R a_{Q}^{* 0.53}$ (dashed line) found by CA6 for dipolar dynamos with rigid boundaries. For hydrodynamic convection with free-slip boundaries, Christensen (2002) suggested a possible asymptotic scaling $N u^{*}=0.077 R a_{Q}^{* 5 / 9}$ in the limiting case of $E \rightarrow 0$. In relatively thinner shells $\left(r_{i} / r_{o}=0.6\right)$, Gastine and Wicht (2012) report $N u^{*}=0.086\left(\left\langle R a_{Q}^{*}\right\rangle_{\rho}\right)^{0.53}$, where $\langle\ldots\rangle_{\rho}$ designates mass-averaged quantities, for density stratified anelastic hydrodynamic convection simulations with free-slip boundaries. All these scaling relations are very close to each other, which suggests that magnetic field, mechanical boundary conditions or density stratification have no substantial effect on the scaling behavior of heat transport in rotating spherical shell convection. However, we note that for larger values 


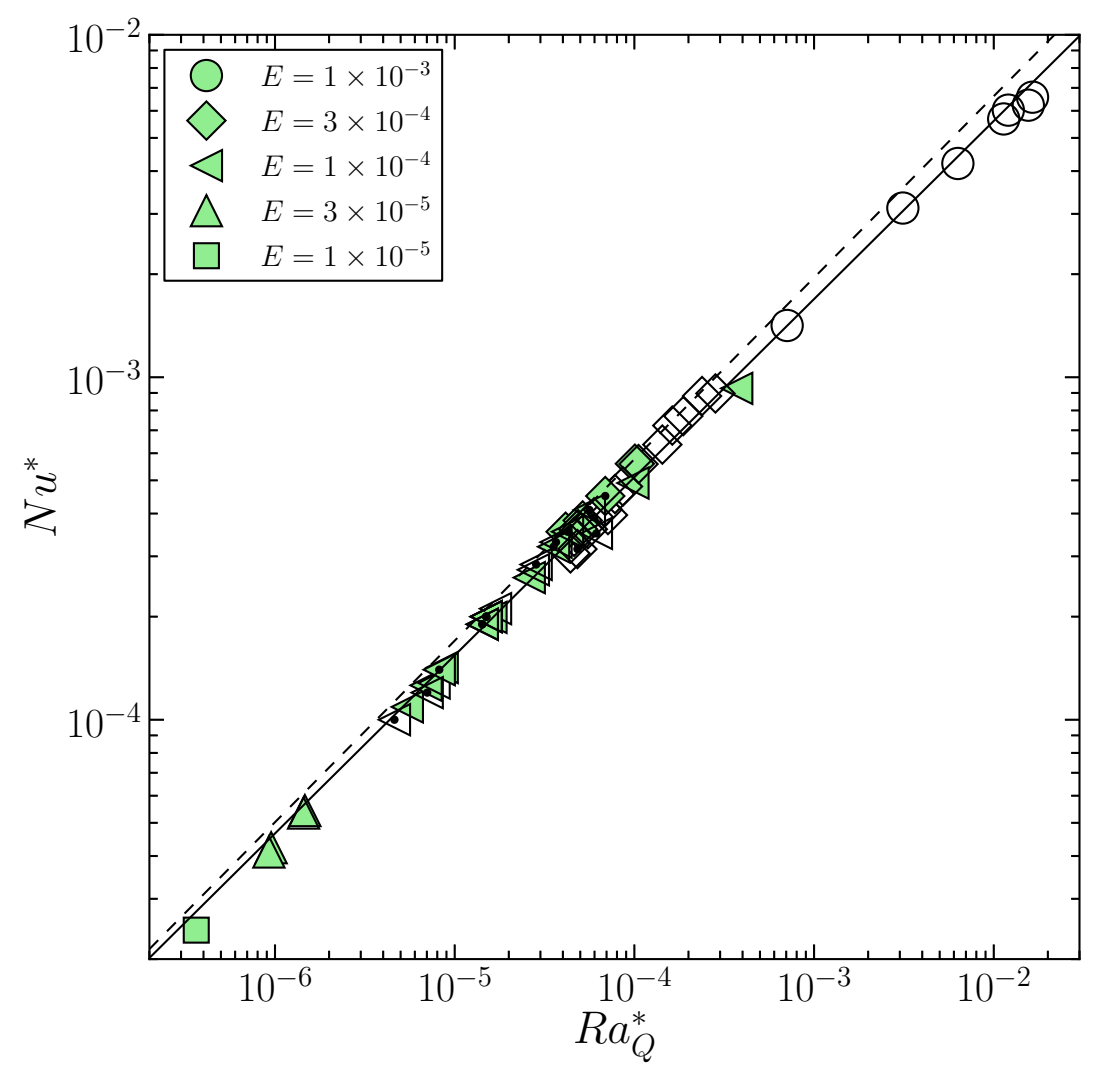

Figure 2.3: Modified Nusselt number versus heat flux based Rayleigh number. The data symbols carrying a thick black dot are adopted from Schrinner et al. (2012). The solidline is a line-fit to data and the dashed-line is the scaling reported by CA6 for dipolar rigid boundary dynamos.

of the Rossby number than the ones considered here, in a regime where inertia dominates over the Coriolis force, the power-law scaling between $N u^{*}$ and $R a_{Q}^{*}$ breaks down (King et al. 2009, 2010, Schmitz and Tilgner 2010).

\subsubsection{Rossby number scaling}

In Fig. 2.4 we plot $R o$ as a function of $R a_{Q}^{*}$. The data points are slightly more scattered as compared to Fig. 2.3, but a consistent scaling is nonetheless evident. Moreover, a somewhat different scaling for dipolar and multipolar dynamos is visible as demonstrated by the two different solid lines. These lines are $R o=0.73 R a_{Q}^{* 0.39}$ (dipolar) and $R o=1.79 R a_{Q}^{* 0.44}$ (multipolar). The scaling reported by CA6 for dipolar rigid boundary dynamos is $R o=0.85 R a_{Q}^{* 0.41}$ (dashed-line), which agrees with our dipolar dynamo scaling.

Similar to CA6, the scatter in Fig. 2.4 can be reduced to some extent by assuming an additional $P m$ dependence. A two-parameter least-square-optimized fit provides $R o=$ 


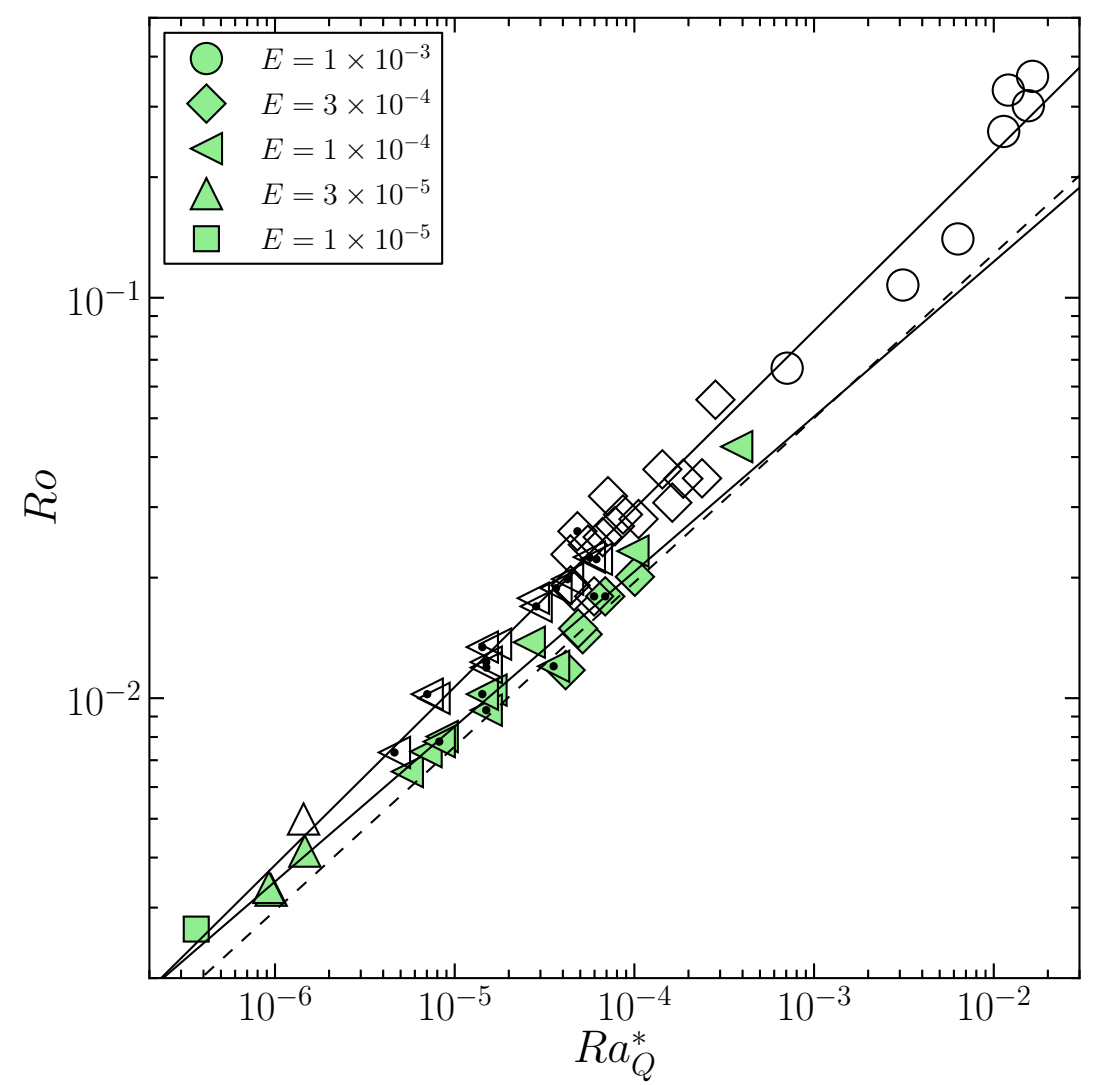

Figure 2.4: Rossby number versus the heat flux based Rayleigh number. The two solid lines are best-fitting lines to dipolar and multipolar dynamos. The dashed-line represent the scaling reported by CA6 for dipolar rigid boundary dynamos.

$0.99 R a_{Q}^{* 0.41} \mathrm{Pm}^{-0.1}$ (dipolar) and $\mathrm{Ro}=2.44 \mathrm{Ra}_{Q}^{*}{ }^{0.47} \mathrm{Pm}^{-0.14}$ (multipolar). This optimization reduces the standard error by almost $48 \%$ in the dipolar scaling and $20 \%$ in the multipolar scaling (see Table 2.2). We also considered the Ekman number as additional parameter for improving the fit, but, as observed by CA6, the resulting exponents are rather small as compared to Ro and $\mathrm{Pm}$ exponents. Hence, we discard a dependence on $E$ in our scaling analysis. The Pm exponent is small and appears to depend on the nature of the magnetic field. The latter could be an artifact of the relatively small size of the data set, especially for dipolar dynamos. In fact, similar to CA6, assuming a scaling of the form $R o \propto\left(R a_{Q}^{*} P m^{-1 / 3}\right)^{\alpha}$, a good fit is obtained for both dipolar and multipolar cases, with somewhat different values for $\alpha$ (see Table 2.2): this form is shown in Fig. 2.5.

As described in Sec. 2.3.1, zonal flows are stronger in rotating convective shells with free-slip mechanical boundaries. In the corresponding MHD systems, dynamos with multipolar magnetic fields will have stronger zonal flows as compared to those with dipolar magnetic fields. This effect is visible in Fig. 2.6 whose top panel shows $R o_{\text {non-zonal }}$ versus $R a_{Q}^{*}$, and the bottom panel shows $R o_{z o n a l}$ versus $R a_{Q}^{*}$. $R o_{\text {non-zonal }}$ and $R o_{z o n a l}$ are calculated by considering the rms velocity excluding the axisymmetric zonal-flow component 


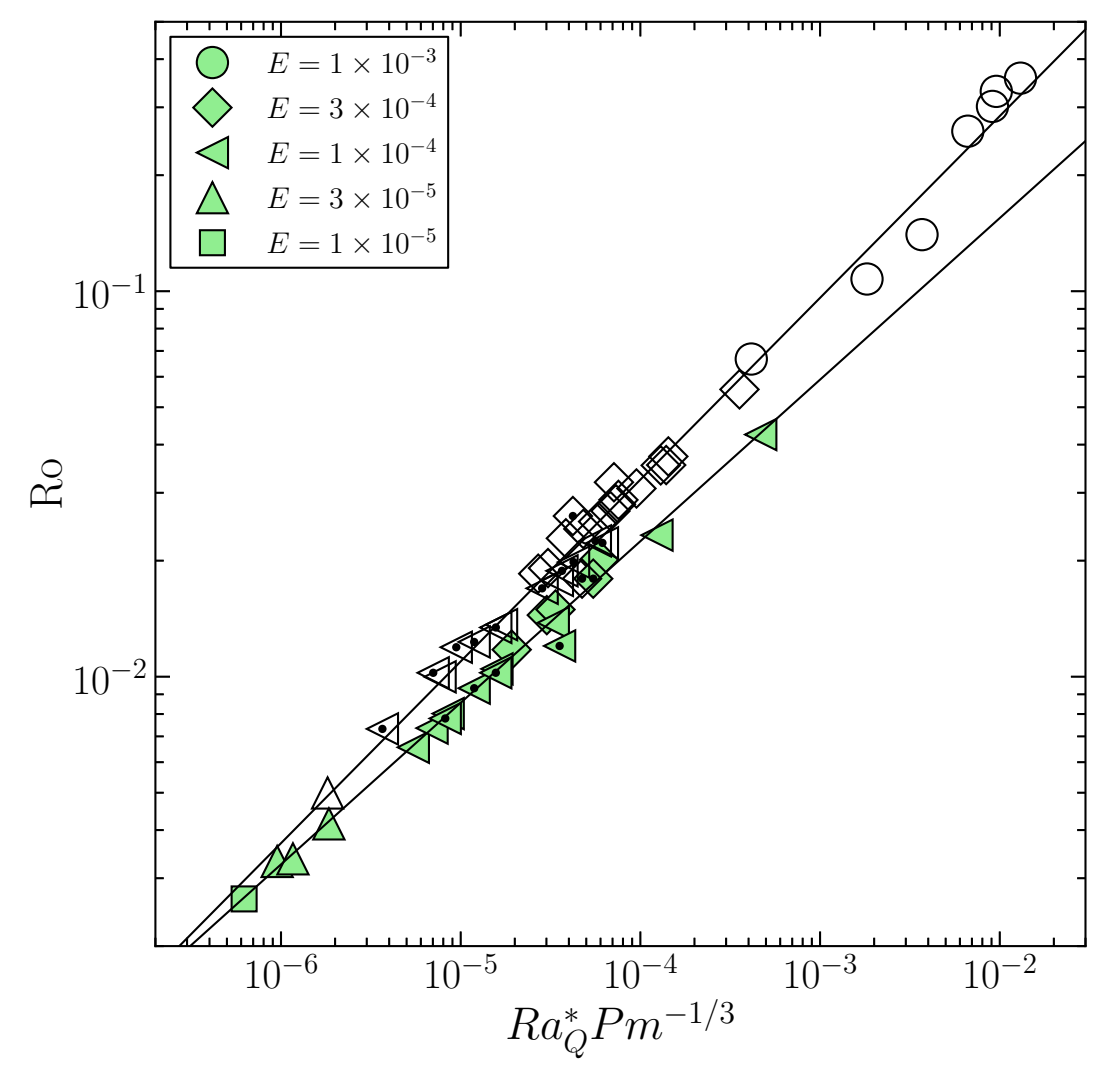

Figure 2.5: Rossby number scaling incorporating a $P m$ dependence.

and the rms velocity of only the axisymmetric zonal flow, respectively. The scaling in Fig. 2.6a is $R o_{\text {non-zonal }}=1.37 R a_{Q}^{* 0.44}$ and in Fig. $2.6 \mathrm{~b}$ is $R o_{\text {zonal }}=0.32 R a_{Q}^{* 0.44}$ (dipolar) and $R o_{\text {zonal }}=0.73 R a_{Q}^{* 0.4}$ (multipolar) We also considered $P m$ as an additional scaling parameter (see Table 2.2). The resulting scaling is marginally better, but the improvement is not as remarkable as it is in Fig. 2.5.

As illustrated in Fig. 2.6a the non-zonal flow component is unaffected by magnetic field geometry as both dipolar and multipolar dynamos follow the same $R o_{\text {non-zonal }}$ scaling. To further investigate the effect of magnetic field on the flow, Fig. 2.6 also incorporates results (gray filled symbols) of hydrodynamic convection in spherical shells with free-slip boundaries from Christensen (2002). This reveals that magnetic field itself does not affect the scaling behavior of $R o_{\text {non-zonal }}$. Coupled with our earlier observation that $N u^{*}$ scaling is effectively same in hydrodynamic and magnetohydrodynamic convection in spherical shells, we can conjecture that the scaling of the flow component which is responsible for heat transfer is unaffected by the presence of magnetic field.

Unlike the non-zonal Rossby number, the zonal Rossby number of dipolar dynamos is consistently lower than that of corresponding multipolar dynamos (Fig. 2.6b). This difference in zonal flows explains the offset in the Rossby number scaling in dipolar and multipolar dynamos seen in Fig. 2.4 and 2.5. Note that as compared to the dipolar branch 

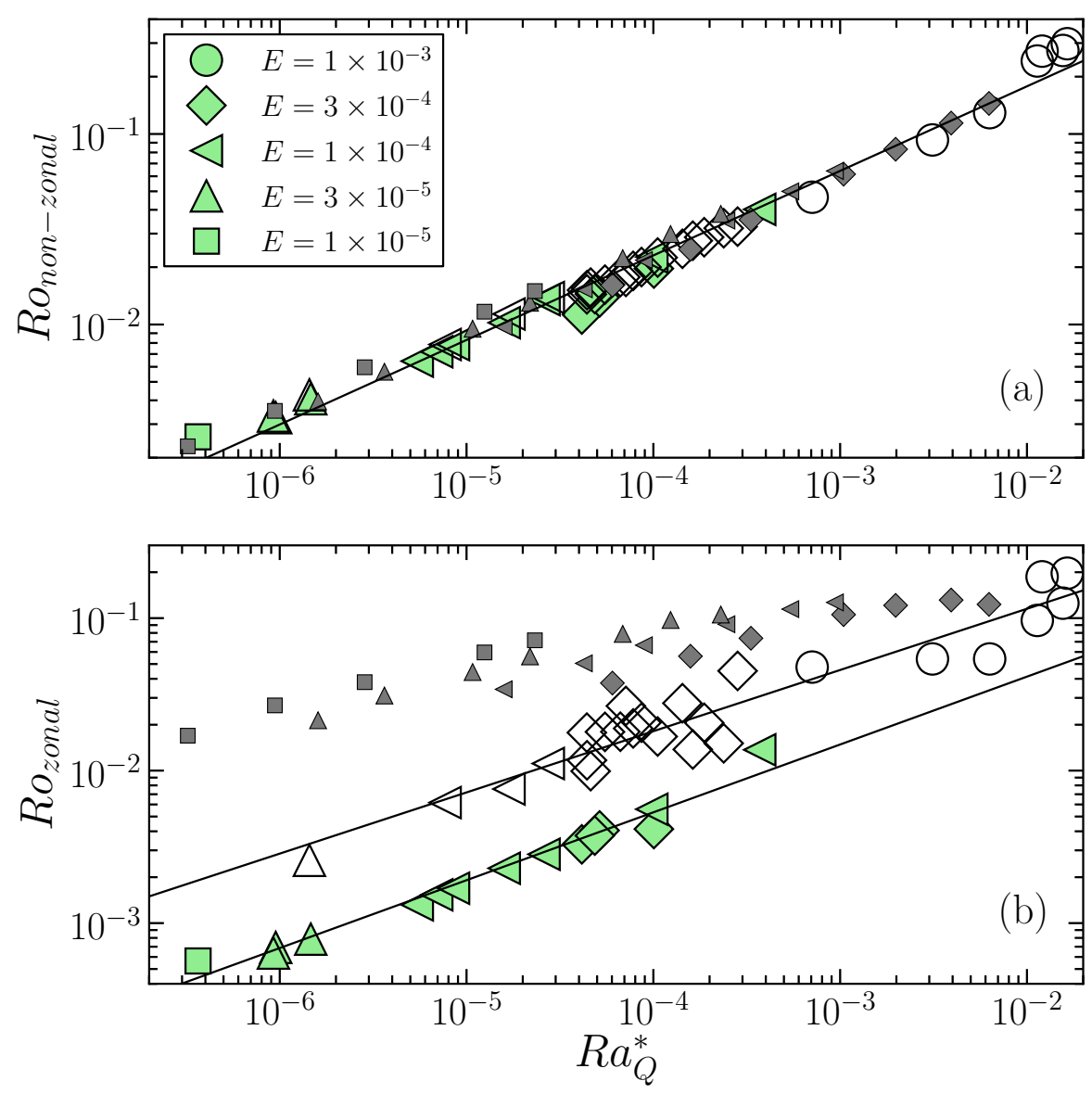

Figure 2.6: Non-zonal Rossby number in (a) and zonal Rossby number in (b) versus the heat flux based Rayleigh number. The gray filled data points are simulation results $(\mathrm{Nu}>2)$ of hydrodynamic free-slip convection from Christensen (2002).

the scatter in the multipolar branch of Fig. $2.6 \mathrm{~b}$ is large. This could be due to the fact that unlike the dipolar branch the multipolar branch is a blend of dynamos which have quadrupolar, octupolar, and sometimes even higher order modes as the most dominating magnetic mode. Since the Maxwell stresses are dependent on the magnetic field geometry, the zonal flows saturate at many different levels. The hydrodynamic zonal flow is consistently higher than both dipolar and multipolar cases. For dipolar dynamos with rigid boundaries, Aubert (2005) argued that Lorentz forces are essential to saturate the zonal flow and bring it into a thermal wind balance, rather than the boundary friction. In purely hydrodynamic cases with free-slip boundaries, it must be viscous friction in the bulk volume that limits the amplitude of the zonal flow. Even for our multipolar dynamos, the zonal flow amplitude is smaller than in the hydrodynamic cases (Fig. 2.6b), which indicates that Lorentz forces play an important role for saturating the flow. For the dipolar dynamos with stronger magnetic fields the damping effect is more pronounced. 


\subsubsection{Magnetic field scaling}

The Ohmic dissipation time $\tau_{m a g}$, which is the ratio of magnetic energy and Ohmic dissipation, is a function of the typical length scale of the magnetic field. As the magnetic Reynolds number is increased, the magnetic field becomes smaller scaled, and, since small scales are associated with faster time scales, the Ohmic dissipation time scale decreases. This qualitative argument was verified by Christensen and Tilgner (2004) in rigid boundary spherical shell dynamos. They showed that $\tau_{\text {mag }}$ (normalized with magnetic diffusion time) is approximately inversely proportional to the magnetic Reynolds number $R m$. When $\tau_{m a g}^{\prime}$ is the Ohmic dissipation time expressed in units of rotation period of the spherical shell, this inverse relation translates to $\tau_{m a g}^{\prime} \propto 1 /$ Ro. In Fig. 2.7 we plot $\tau_{m a g}^{\prime}$ versus Ro. A best-fit line to this data set suggests $\tau_{\text {mag }}^{\prime} \propto 1 / R o^{0.8}$. Since the scatter in Fig. 2.7 is substantial, the difference between the exponents -0.8 and -1 may not be very significant. Christensen (2010) have discussed a more complex scaling for $\tau_{\text {mag }}$ and report a marginal improvement in the quality of the fit. Although the inset figure shows a small decrease in $\tau_{\text {mag }}^{\prime}$ for bistable states when the magnetic field is multipolar, the scalings for dipolar and multipolar dynamos appear to follow the same trend. Moreover, if we plot $\tau_{\text {mag }}^{\prime}$ versus $R o_{\text {zonal }}$ or $R o_{\text {non-zonal }}$ (not shown), then the scatter is increased as compared to Fig. 2.7. It highlights that the important parameter in the context of ohmic dissipation is the total Rossby number, which incorporates the zonal-flow contribution.

CA6 argue that the magnetic field strength might be determined by the power available to balance the Ohmic dissipation. Following this argument, the Lorentz number should be accordingly corrected by the Ohmic fraction $f_{\text {ohm }}$ which is the ratio of Ohmic dissipation and the power generated via buoyancy forces. In Fig. 2.8, we plot the corrected Lorentz number versus the flux-based Rayleigh number $R a_{Q}^{*}$, which is a nondimensional measure for the power generated by the action of buoyancy forces (CA6). A best fit is obtained by $L o / f_{\text {ohm }}^{1 / 2}=1.08 R a_{Q}^{* 0.37}$ (dipolar) and $L o / f_{\text {ohm }}^{1 / 2}=0.65 R a_{Q}^{* 0.35}$ (multipolar). The dipolar scaling in Fig. 2.8 is close to the rigid boundary dipolar scaling $L o / f_{\text {ohm }}^{1 / 2}=0.92 R a_{Q}^{* 0.34}$ (dashed line) reported by CA6. Furthermore, a two-parameter optimized fit for dipolar dynamos is $L o / f_{\text {ohm }}^{1 / 2}=0.72 \mathrm{Ra}_{Q}^{*}{ }^{0.33} \mathrm{Pm}^{0.14}$ and for multipolar dynamos is $L o / f_{o h m}^{1 / 2}=0.51 R a_{Q}^{* 0.33} \mathrm{Pm}^{0.11}$. The inclusion of $P m$ reduces the standard error by almost $67 \%$ (dipolar) and 39\% (multipolar). Again, assuming a simplified form $L o / f_{o h m}^{1 / 2} \propto\left(R a_{Q}^{*} P m^{1 / 3}\right)^{\beta}$, the quality of the fit is hardly reduced (Table 2.2, Fig. 2.9). A cursory inspection of Figs. 2.8 and 2.9 suggests that the dipolar and multipolar scalings are almost the same except for an offset in the pre-factor by $\approx 8 / 5$.

An inverse relation of $\tau_{\text {mag }}^{\prime}$ and $R o$ translates to $L o / f_{o h m}^{1 / 2} \propto \sqrt{R a_{Q}^{*} / R o}$. If we now substitute the $R o$ scaling from Fig. 2.4 in the previous relation, then an offset of $\approx 8 / 5$ is indeed expected for dipolar and multipolar scaling. The $\tau_{\text {mag }}^{\prime} \propto 1 /$ Ro argument therefore supports the offset in the scaling observed in Fig. 2.8 to a good extent. Schrinner et al. (2012) have also reported a similar shift in their free-slip dynamo simulations; they qualitatively argue that the offset in the scaling is due to decrease in the $f_{\text {ohm }}$ in multipolar dynamo cases. In the case of rigid boundaries, Christensen (2010) also reported a smaller scaling pre-factor of the Lorentz number for multipolar dynamos compared to dipolar ones. Our inspection of data from earlier spherical shell dynamos with rigid boundaries reveals that $R o$ for both dipolar and multipolar dynamos follows same scaling relation, 


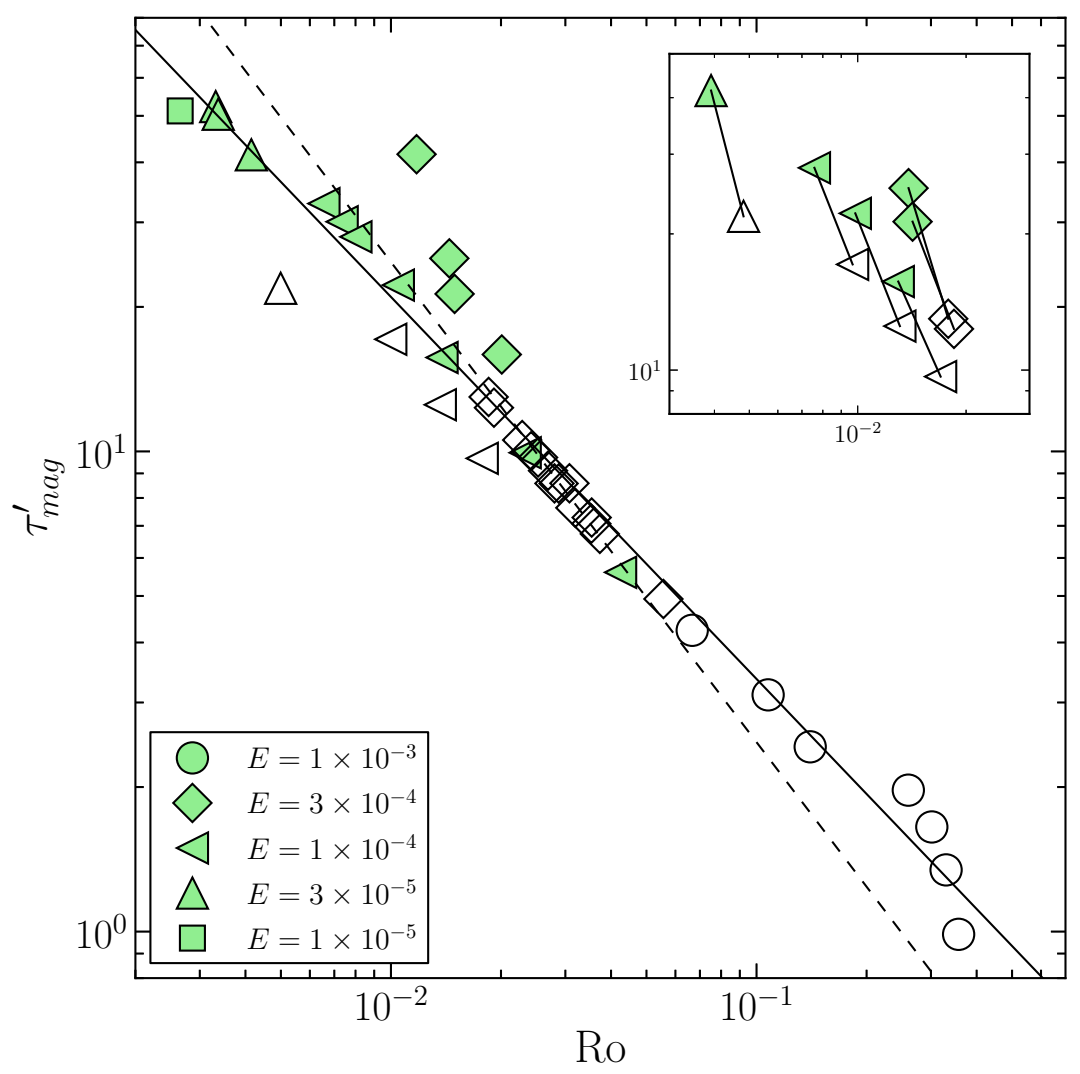

Figure 2.7: Ohmic dissipation time versus the Rossby number. Solid line represents a best fit line, while the dashed line represents $\tau_{\text {mag }}^{\prime} \propto 1 / R o$. The inset-figure contains bistable pairs connected by solid lines.

unlike what we observed. Clearly, more analysis is required to conclusively demonstrate the reason for such an offset.

\subsection{Discussion and conclusions}

In this article we investigated the effect of free-slip mechanical boundaries on various scaling laws in spherical shell dynamos. We compared the inferred scaling laws with earlier reported scalings for rigid boundary dynamos.

We observed bistability, i.e. dipolar and multipolar dynamos coexisting for same control parameters. This agrees with the earlier findings (Simitev and Busse 2009, 2012, Sasaki et al. 2011, Schrinner et al. 2012, Gastine et al. 2012) and reinforces the importance of free-slip boundaries and zonal flows for this phenomenon. Our solar system giant planets are expected to have low local Rossby numbers $\left(R o_{l}<0.1\right)$ (Olson and Christensen 2006). Noting that free-slip boundaries are more appropriate for modeling these giant planets, bistability could be the reason why Jupiter and Saturn have dipole 


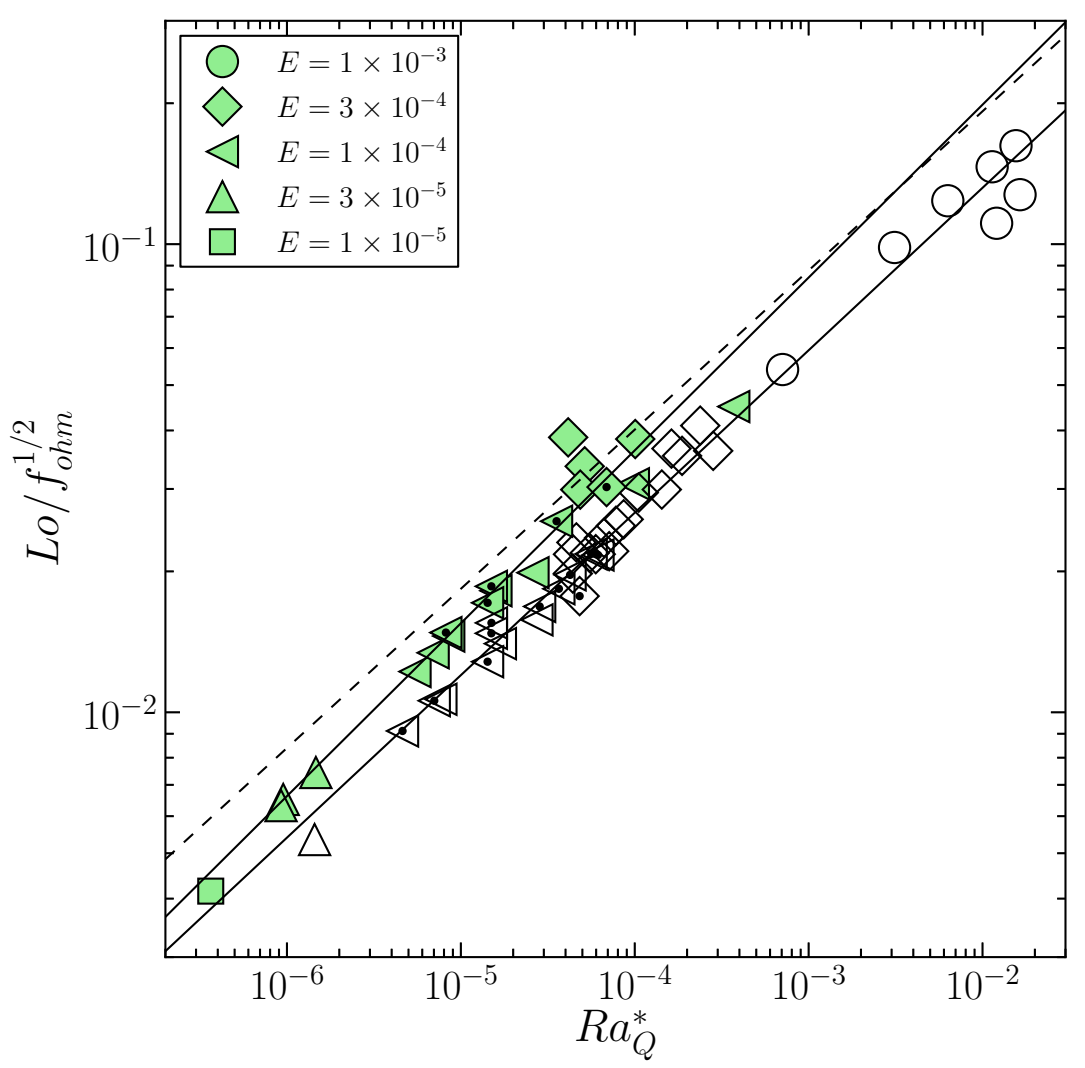

Figure 2.8: Lorentz number versus the heat flux based Rayleigh number. The two solid lines are best-fitting lines to dipolar and multipolar dynamos. The dashed-line represent the scaling reported by CA6 for dipolar rigid boundary dynamos.

dominated magnetic fields while Uranus and Neptune have multipolar magnetic fields.

The modified Nusselt number scales as $N u^{*}=0.061 R a_{Q}^{* 0.52}$ which is very close to the scaling for rigid boundary dynamos (Christensen and Aubert 2006) and non-magnetic convection in spherical shell with free-slip boundaries (Christensen 2002). At values of the Rayleigh number that are higher than in our simulations, a gradual transition to a weaker dependence of the Nusselt number on the Rayleigh number in expected (King et al. 2009, 2010, Schmitz and Tilgner 2010), which can be associated with a change from a rotationally-dominated regime to a non-rotating regime. A matter of debate is whether the relative thickness of Ekman-layer and thermal boundary layer plays a role in this transition (King et al. 2009). Schmitz and Tilgner (2010) dispute this boundary layer hypothesis because they observe that the transition occurs similarly for both rigid and free-slip boundaries. Because our simulations do not reach the transition point, they can not contribute to this ongoing discussion.

The Rossby number scales as $R o=0.73 R a_{Q}^{* 0.39}$ for dipolar and as $R o=1.79 R a_{Q}^{* 0.44}$ for multipolar dynamos. The offset in the scaling of dipolar and multipolar dynamos can be attributed to different zonal flow characteristics: zonal flow quenching is stronger in 


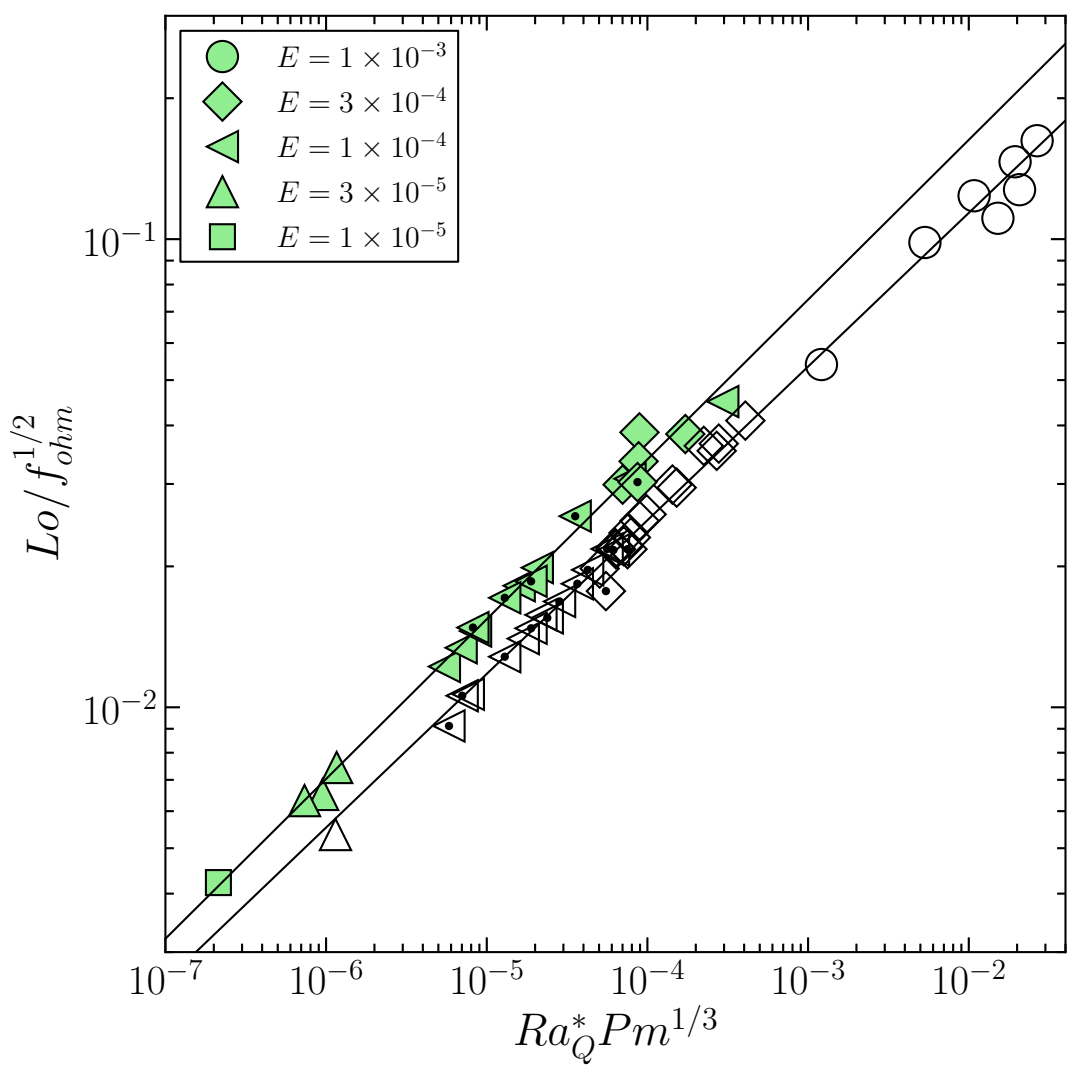

Figure 2.9: Lorentz number scaling incorporating a $P m$ dependence.

a dipolar magnetic field configuration as compared to a multipolar configuration. This results in a smaller pre-factor in the Rossby number scaling for dipolar dynamos. We also used earlier numerical results of hydrodynamic convection in spherical shells with free-slip boundaries (Christensen 2002) and observed that the non-zonal flow scaling $\left(R o_{\text {non-zonal }}\right)$ is unaffected by the presence of magnetic field while the zonal flow scaling $\left(R o_{\text {zonal }}\right)$ is effectively quenched by magnetic field.

The corrected Lorentz number scales as $L o / f_{\text {ohm }}^{1 / 2}=1.08 R a_{Q}^{* 0.37}$ for dipolar dynamos and $L o / f_{\text {ohm }}^{1 / 2}=0.65 R a_{Q}^{*} 0.35$ for multipolar dynamos. The exponents are almost identical but the pre-factors differ. We investigated the origin of such shifted scaling and found that, using the scalings for Rossby number (inferred from our data-set) and an inverse relationship between ohmic dissipation time and magnetic Reynolds number, parallel and shifted scalings for $L o / f_{o h m}^{1 / 2}$ are indeed expected. The observed and the expected ratio of dipolar and multipolar $L o / f_{o h m}^{1 / 2}$ scalings agreed quite well. This agreement suggests that zonal flow amplitude controls the final mean magnetic field strength of the dynamos with free-slip boundaries.

Similar to Christensen and Tilgner (2004) and Christensen and Aubert (2006), we also observed that a small dependence on the magnetic Prandtl number improves the quality of 
Table 2.2: The various scaling laws inferred from our study, along with the crosscorrelation coefficient $R$ and the Standard error (standard deviation divided by square-root of number of data points).

\begin{tabular}{lcc}
\hline \hline Scaling & $R$ & Standard-error \\
\hline$N u^{*}=0.061 R a_{Q}^{* 0.52}$ & 0.9987 & 0.0036 \\
$R o_{\text {non-zonal }}=1.37 R a_{Q}^{* 0.44}$ & 0.9924 & 0.0089 \\
$R o_{\text {non-zonal }}=1.99\left(R a_{Q}^{*} P m^{-1 / 3}\right)^{0.47}$ & 0.9937 & 0.0086 \\
\hline Dipolar & & \\
$R o=0.73 R a_{Q}^{* 0.39}$ & 0.9903 & 0.0128 \\
$R o=0.99 R a_{Q}^{* 0.41} P m^{-0.1}$ & 0.9976 & 0.0067 \\
$R o=1.07\left(R a_{Q}^{*} P m^{-1 / 3}\right)^{0.42}$ & 0.9966 & 0.0082 \\
$R o_{\text {zonal }}=0.32 R a_{Q}^{* 0.44}$ & 0.9873 & 0.0198 \\
$R o_{\text {zonal }}=0.47\left(R a_{Q}^{*} P m^{-1 / 3}\right)^{0.48}$ & 0.9896 & 0.0192 \\
$L o / f_{\text {ohm }}^{1 / 2}=1.08 R a_{Q}^{* 0.37}$ & 0.9820 & 0.0167 \\
$L o / f_{\text {ohm }}^{1 / 2}=0.71 R a_{Q}^{* 0.33} P m^{0.14}$ & 0.9972 & 0.0059 \\
$L o / f_{\text {ohm }}^{1 / 2}=0.78\left(R a_{Q}^{*} P m^{1 / 3}\right)^{0.34}$ & 0.9967 & 0.0066 \\
\hline$M u l t i p o l a r$ & & \\
$R o=1.79 R a_{Q}^{* 0.44}$ & 0.9916 & 0.0098 \\
$R o=2.44 R a_{Q}^{* 0.47} P^{-0.14}$ & 0.9954 & 0.0078 \\
$R o=2.49\left(R a_{Q}^{*} P m^{-1 / 3}\right)^{0.47}$ & 0.9952 & 0.0078 \\
$R o_{\text {zonal }}=0.73 R a_{Q}^{* 0.4}$ & 0.9553 & 0.0259 \\
$R o_{\text {zonal }}=1.05\left(R a_{Q}^{*} P m^{-1 / 3}\right)^{0.43}$ & 0.9699 & 0.0226 \\
$L o / f_{\text {ohm }}^{1 / 2}=0.65 R a_{Q}^{* 0.35}$ & 0.9941 & 0.0064 \\
$L o / f_{\text {ohm }}^{1 / 2}=0.51 R a_{Q}^{* 0.33} P m^{0.11}$ & 0.9975 & 0.0039 \\
\hline \hline
\end{tabular}

the scalings, especially in the dipolar dynamo cases. However, results form the Karlsruhe laboratory dynamo experiment motivated Christensen and Tilgner (2004) to conjecture that such small Pm dependence might disappear when $P m<<1$. The lowest $P m$ in our study is of order unity, which makes it difficult to ascertain this conjecture. So far, our free-slip simulations and the rigid boundary simulation results of Christensen and Aubert (2006) support scalings which have some $P m$ contribution. Simulations which attain $P m<<1$ will shed more light on this issue.

Objects such as stars and giant planets have free-surface flows, very high density stratification, and probably fully convective interiors. Nonetheless, scaling laws inferred from Boussinesq dynamo models with rigid boundaries that have been tailored to model the geodynamo, have been applied with some success also to giant planets and rapidly rotating stars (Christensen et al. 2009, Christensen 2010). Similar scaling of physical properties despite such drastic physical differences is puzzling. As a first step toward testing the scaling laws for conditions that are more applicable to giant planets and stars, we studied here the influence of the mechanical boundary conditions. Our analysis shows that the boundary conditions do not substantially affect the scaling behavior of the rms velocity and the magnetic field strength, which supports the validity of the original scaling laws for a broader class of objects. Future simulations of dynamos with density stratification 
and fully convective interiors will address the remaining critical factors.

\section{Acknowledgements}

We are grateful to Martin Schrinner for providing extra information about his free-slip dynamo simulations. We thank Julien Aubert and another anonymous referee for very stimulating and helpful comments. We acknowledge funding from the Deutsche Forschungsgemeinschaft (DFG) through Project SFB 943 / A17 and through the special priority program 1488 (PlanetMag, http://www.planetmag.de) of the DFG. All the figures were generated using matplotlib (www.matplotlib.org). 


\section{Bibliography}

Aubert, J. (2005). Steady zonal flows in spherical shell dynamos. Journal of Fluid Mechanics, 542(1), 53-67.

Aubert, J., Brito, D., Nataf, H.-C., Cardin, P., and Masson, J.-P. (2001). A systematic experimental study of rapidly rotating spherical convection in water and liquid gallium. Phys. Earth Planet. Int., 128(1-4), 51-74.

Aubert, J., Labrosse, S., and Poitou, C. (2009). Modelling the palaeo-evolution of the geodynamo. Geophys. J. Int., 179(3), 1414-1428.

Browning, M. K. (2008). Simulations of dynamo action in fully convective stars. Astrophys. J., 676(2), 1262-1280.

Christensen, U. R. (2002). Zonal flow driven by strongly supercritical convection in rotating spherical shells. J. Fluid Mech., 470, 115-133.

Christensen, U. R. (2010). Dynamo scaling laws and applications to the planets. Space Sci. Rev., 152, 565-590.

Christensen, U. R. and Aubert, J. (2006). Scaling properties of convection-driven dynamos in rotating spherical shells and application to planetary magnetic fields. Geophys. J. Int., 166(1), 97-114.

Christensen, U. R. and Tilgner, A. (2004). Power requirement of the geodynamo from ohmic losses in numerical and laboratory dynamos. Nature, 429, 169-171.

Christensen, U. R., Olson, P., and Glatzmaier, G. A. (1999). Numerical modelling of the geodynamo: a systematic parameter study. Geophys. J. Int., 138(2), 393-409.

Christensen, U. R., Holzwarth, V., and Reiners, A. (2009). Energy flux determines magnetic field strength of planets and stars. Nature, 457(7226), 167-169.

Ferraro, V. (1937). The non-uniform rotation of the sun and its magnetic field. Mon. Not. Roy. Astron. Soc., 97, 458-472.

Gastine, T. and Wicht, J. (2012). Effects of compressibility on driving zonal flow in gas giants. Icarus, 219(1), $428-442$.

Gastine, T., Duarte, L., and Wicht, J. (2012). Dipolar versus multipolar dynamos: the influence of the background density stratification. $A \mathcal{E} A, \mathbf{5 4 6}, \mathrm{A} 19$. 
Gastine, T., Morin, J., Duarte, L., Reiners, A., Christensen, U., and Wicht, J. (2013).

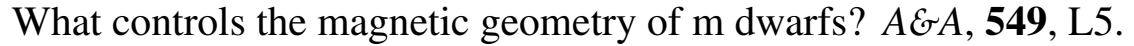

Glatzmaier, G. A. and Roberts, P. H. (1995a). A three-dimensional convective dynamo solution with rotating and finitely conducting inner core and mantle. Phys. Earth Planet. Int., 91, 63-75.

Glatzmaier, G. A. and Roberts, P. H. (1995b). A three dimensional self consistent computer simulation of a geomagnetic field reversal. Nature, 377, 203-209.

Grote, E. and Busse, F. H. (2000). Hemispherical dynamos generated by convection in rotating spherical shells. Phys. Rev. E, 62(3), 4457-4460.

Grote, E., Busse, F. H., and Tilgner, A. (1999). Convection-driven quadrupolar dynamos in rotating spherical shells. Phys. Rev. E, 60(5), R5025-8.

Grote, E., Busse, F. H., and Tilgner, A. (2000). Regular and chaotic spherical dynamos. Phys. Earth Planet. Int., 117, 259-272.

Jones, C. A. (2011). Planetary magnetic fields and fluid dynamos. Annual Rev. of Fluid Mech., 43(1), 583-614.

Kageyama, A. and Sato, T. (1995). Computer simulation of a magnetohydrodynamic dynamo. II. Phys. of Plasmas, 2(5), 1421-1431.

King, E. M., Stellmach, S., Noir, J., Hansen, U., and Aurnou, J. M. (2009). Boundary layer control of rotating convection systems. Nature, 457(7227), 301-4.

King, E. M., Soderlund, K. M., Christensen, U. R., Wicht, J., and Aurnou, J. M. (2010). Convective heat transfer in planetary dynamo models. Geochem. Geophys. Geosys., 11(6), 1-19.

Kuang, W. and Bloxham, J. (1997). An Earth-like numerical dynamo model. Nature, 389(September), 371-374.

Kuang, W. and Bloxham, J. (1999). Numerical modeling of magnetohydrodynamic convection in a rapidly rotating spherical shell: weak and strong field dynamo action. $J$. Comput. Phys., 153(1), 51-81.

Miyagoshi, T., Kageyama, A., and Sato, T. (2010). Zonal flow formation in the Earth's core. Nature, 463(7282), 793-6.

Olson, P. and Christensen, U. R. (2006). Dipole moment scaling for convection-driven planetary dynamos. Earth and Planet. Sci. Lett., 250(3-4), 561 - 571.

Sasaki, Y., Takehiro, S.-i., Kuramoto, K., and Hayashi, Y.-Y. (2011). Weak-field dynamo emerging in a rotating spherical shell with stress-free top and no-slip bottom boundaries. Phys. Earth Planet. Int., 188(3-4), 203-213.

Schmitz, S. and Tilgner, A. (2010). Transitions in turbulent rotating rayleigh-bénard convection. Geophys. Astrophys. Fluid Dyn., 104(5-6), 481-489. 


\title{
3 Consistent scaling laws in anelastic spherical shell dynamos
}

\begin{abstract}
Numerical dynamo models always employ parameter values that differ by orders of magnitude from the values expected in natural objects. However, such models have been successful in qualitatively reproducing properties of planetary and stellar dynamos. This qualitative agreement fuels the idea that both numerical models and astrophysical objects may operate in the same asymptotic regime of dynamics. This can be tested by exploring the scaling behavior of the models. For convection-driven incompressible spherical shell dynamos with constant material properties, scaling laws had been established previously that relate flow velocity and magnetic field strength to the available power. Here we analyze 273 direct numerical simulations using the anelastic approximation, involving also cases with radius-dependent magnetic, thermal and viscous diffusivities. These better represent conditions in gas giant planets and low-mass stars compared to Boussinesq models. Our study provides strong support for the hypothesis that both mean velocity and mean magnetic field strength scale as a function of power generated by buoyancy forces in the same way for a wide range of conditions ${ }^{1,2}$.
\end{abstract}

\subsection{Introduction}

Dynamo simulations aim to capture the magnetic field generation process in planetary and stellar interiors and have been very successful in qualitatively reproducing many of the observed properties (Brun et al. 2004, Wicht and Tilgner 2010, Jones 2011). However, all such numerical simulations use inconsistent control parameters, either too large or too small, due to limited computational resources. As a consistency check between our theoretical understanding of the dynamo mechanism with astrophysical observations, it is of paramount importance to establish generic scaling laws which are valid in the relevant dynamical regimes.

Many attempts have been made earlier to derive a scaling theory for the mean magnetic field, for example, on force balance considerations (Stevenson 1979, Curtis and Ness

\footnotetext{
${ }^{1}$ The content of this chapter has been reproduced from: R. K. Yadav, T. Gastine, U. R. Christensen, and L. Duarte, "Consistent scaling laws in anelastic spherical shell dynamos", ApJ 774, 6, 2013. DOI: 10.1088/0004-637X/774/1/6.

${ }^{2}$ Contribution: I ran part of the simulations used in the study but the majority of the simulations were borrowed from earlier studies by co-authors Thomas Gastine and Lucia Duarte. I carried out the analysis presented in the paper. I wrote the first draft of the manuscript; despite changes in later revisions the original structure of the manuscript was preserved.
} 
1986, Mizutani et al. 1992, Sano 1993, Starchenko and Jones 2002). However, none of the suggested scaling laws was generally accepted. Progress was made in this regard due to the increase in computational power in the last decade. Christensen and Aubert (2006) analyzed the results of parameter studies of dynamo simulations under the Boussinesq approximation in a rotating spherical shell and found that mean velocity and mean magnetic field scale as a function of the available convective power generated via buoyancy forces. The power-based scaling laws (hereafter referred to as PBS) do not explicitly depend on rotation rate in case of magnetic field scaling. In the parameter range of the simulations, a secondary influence of diffusivities in the form of magnetic Prandtl number (ratio of viscosity and magnetic diffusivity) was found.

Subsequent studies generalized the PBS to Boussinesq dynamos with different physical setups and different boundary conditions (Takahashi et al. 2008, Aubert et al. 2009, Christensen 2010, Schrinner et al. 2012, Yadav et al. 2013). Olson and Christensen (2006) derived PBS for the magnetic dipole moment from numerical simulations and found an order of magnitude agreement with dynamos in solar system planets. Furthermore, Christensen et al. (2009) and Christensen (2010) observed a good agreement between PBS of magnetic field from numerical simulations and observationally constrained magnetic field of Earth, Jupiter, rapidly rotating low-mass stars, and possibly Uranus and Neptune. Stelzer and Jackson (2013) maintain that including a dependence on the magnetic Prandtl number is mandatory for an adequate fit, at least in the parameter range of current numerical simulations. Davidson (2013) recently provided interesting theoretical arguments supporting the power based scaling laws.

The agreement between the prediction of scaling laws derived from Boussinesq numerical simulations and observed magnetic fields in low-mass stars is rather puzzling. Unlike the dynamo mechanism in solar type stars, where the strong differential rotation and magnetic field generation by shear at the tachocline are thought to be a key ingredient (Ossendrijver 2003), rapidly rotating low-mass stars (mass $<0.35 M_{\odot}$ ) and giant planets possibly harbor dynamos similar to the geodynamo, where helical convection columns aligned with the rotation axis are instrumental. However, the hydrogen rich interiors of low-mass stars are vastly different from the liquid metal interiors of the Earth-like objects. The density and transport properties in the liquid core of the latter vary by some tens of percent (e.g. $\approx 20 \%$ density change across Earth's liquid core; see Braginsky and Roberts (1995)) and can be considered constant. On the other hand, the interiors of gas planets and stars have significant density stratification and transport properties (such as electrical conductivity and thermal diffusivity) may vary by orders of magnitude (French et al. 2012).

The power based scaling laws discussed above were derived from a large number of Boussinesq dynamo simulations. Although density stratified models with radially-varying transport properties have been commonly employed in the stellar dynamo community (see e.g. Gilman and Glatzmaier (1981), Brun et al. (2004), Browning (2008)), a systematic scaling study of important diagnostic quantities has never been carried out. One of the reasons is the substantial increase in computational requirements associated with anelastic density-stratified dynamo simulations which makes parameter studies rather expensive (Jones et al. 2009). For nonmagnetic rotating convection, Gastine and Wicht (2012) found in systematic model studies that the velocity of convection and of zonal flow scales in the same way for Boussinesq and anelastic cases. Here we extend this to dynamo mod- 
els with density stratification. We also include cases with different forms of variation in transport properties, different radial gravity profiles and different mechanical and magnetic boundary conditions. Our aim is not to model any particular class of astrophysical object as realistically as possible, but rather concentrate on generic scaling properties. Our analysis of more than 270 numerical dynamo models shows that the same power based scaling laws apply to a wide variety of dynamos.

\subsection{Equations and numerical setups}

\subsubsection{Anelastic MHD equations}

We consider dynamo action in spherical shells, with inner radius $r_{i}$ and outer radius $r_{o}$, filled with an electrically conducting fluid. The aspect ratio $\eta$ is defined as $r_{i} / r_{o}$. The shell rotates along a vertical axis $\hat{z}$ with constant angular velocity $\Omega$. Convection inside the shell is driven by a fixed entropy contrast $\Delta s$ between the inner and the outer boundary. We work in dimensionless units using shell thickness $D=r_{o}-r_{i}$ and inverse rotation frequency $\Omega^{-1}$ as the fundamental length and time units, respectively. The density $\rho$ and entropy $s$ are non-dimensionalized using $\rho\left(r_{o}\right)=\rho_{o}$ and $\Delta s$, respectively. Magnetic field is scaled by $\Omega D \sqrt{\mu \rho_{o}}$, where $\mu$ is the magnetic permeability.

To model the low-Mach number flows in the density stratified interiors of giant planets and low-mass stars, we employ the anelastic approximation. It allows radial variation of density while filtering out the fast acoustic waves out of the system (Braginsky and Roberts 1995, Lantz and Fan 1999). The anelastic approximation assumes an adiabatic reference state, i.e. $d \tilde{T} / d r=-g / c_{p}$, where $g$ is gravity and $c_{p}$ is the specific heat at constant pressure. The radius-dependent reference state quantities are highlighted with a tilde on top. For the sake of generality we define the gravity profile as

$$
g(r)=g_{1} \frac{r}{r_{o}}+g_{2} \frac{r_{o}^{2}}{r^{2}}
$$

and by using $g_{1}$ or $g_{2}$ appropriately we can either choose a linear gravity profile $\left(g_{1}=1\right.$, $g_{2}=0$ ), approximately representing a self-gravitating body with weak density variation, or an $r^{-2}$ gravity profile $\left(g_{1}=0, g_{2}=1\right)$, exemplifying objects with massive core. Assuming an ideal gas equation of state leads to a polytropic reference state defined by $\tilde{\rho}=\tilde{T}^{m}$, where $m$ is the polytropic index. Solving for $\tilde{T}$ using Eq. (3.1) leads to

$$
\tilde{T}=1-c_{o}\left[\frac{g_{1}}{2}\left(\frac{r^{2}}{r_{o}^{2}}-1\right)+g_{2}\left(1-\frac{r_{o}}{r}\right)\right]
$$

with

$$
c_{o}=\frac{\eta\left(e^{\frac{N_{\rho}}{m}}-1\right)}{\frac{g_{1}}{2}\left(\eta-\eta^{3}\right)+g_{2}(1-\eta)},
$$

where $N_{\rho}=\ln \left(\tilde{\rho}\left(r_{i}\right) / \tilde{\rho}\left(r_{o}\right)\right)$ represents the number of density scale heights across the shell.

The thermodynamic variables, density, pressure, and temperature are then decomposed into the sum of reference state values and small perturbations as $\tilde{\rho}+\rho, \tilde{P}+p$, and 
$\tilde{T}+T$ respectively (Gilman and Glatzmaier 1981, Braginsky and Roberts 1995, Lantz and Fan 1999). The evolution of velocity $\mathbf{u}$ is governed by

$$
\begin{gathered}
\nabla \cdot(\tilde{\rho} \mathbf{u})=0, \\
\frac{\partial \mathbf{u}}{\partial t}+\mathbf{u} \cdot \nabla \mathbf{u}+2 \hat{z} \times \mathbf{u}=-\nabla \frac{p}{\tilde{\rho}}+\frac{\operatorname{Ra} E^{2}}{\operatorname{Pr}} g(r) s \hat{r}+\frac{1}{\tilde{\rho}}(\nabla \times \mathbf{B}) \times \mathbf{B}+\frac{E}{\tilde{\rho}} \nabla \cdot \tilde{v} S,
\end{gathered}
$$

where $\mathbf{B}$ is magnetic field and $\hat{r}$ is the radial unit vector. The traceless rate-of-strain tensor $S$ is defined by

$$
S_{i j}=2 \tilde{\rho}\left(e_{i j}-\frac{1}{3} \delta_{i j} \nabla \cdot \mathbf{u}\right)
$$

with

$$
e_{i j}=\frac{1}{2}\left(\frac{\partial u_{i}}{\partial x_{j}}+\frac{\partial u_{j}}{\partial x_{i}}\right)
$$

where $\delta_{i j}$ is the identity matrix. The entropy $s$ evolves according to

$$
\tilde{\rho} \tilde{T} E\left(\frac{\partial s}{\partial t}+\mathbf{u} \cdot \nabla s\right)=\frac{E^{2}}{P_{r}} \nabla \cdot(\tilde{\kappa} \tilde{\rho} \tilde{T} \nabla s)+\frac{P_{r} c_{o}(1-\eta)}{R a}\left[\tilde{v} Q_{v}+\frac{\tilde{\lambda}}{P_{m}}(\nabla \times \mathbf{B})^{2}\right],
$$

where the viscous heating contribution is

$$
Q_{v}=2 \tilde{\rho}\left[e_{i j} e_{j i}-\frac{1}{3}(\nabla \cdot \mathbf{u})^{2}\right]
$$

The magnetic induction is governed by

$$
\begin{gathered}
\frac{\partial \mathbf{B}}{\partial t}=\nabla \times(\mathbf{u} \times \mathbf{B})-\frac{E}{P_{m}} \nabla \times(\tilde{\lambda} \nabla \times \mathbf{B}), \\
\nabla \cdot \mathbf{B}=0 .
\end{gathered}
$$

In the above equations, kinematic viscosity $v$, thermal diffusivity $\kappa$, and magnetic diffusivity $\lambda$ are normalized by their value at the inner boundary $\left(r=r_{i}\right)$; they are denoted by $\tilde{v}, \tilde{\kappa}$, and $\tilde{\lambda}$. The various dimensionless control parameters appearing in Eqs. (3.4-3.9) are:

$$
\begin{gathered}
\text { Rayleigh number } R a=\frac{g\left(r_{o}\right) D^{3} \Delta s}{c_{p} v_{i} \kappa_{i}}, \\
\text { Ekman number } E=\frac{v_{i}}{\Omega D^{2}}, \\
\text { Prandtl number } P_{r}=\frac{v_{i}}{\kappa_{i}}, \\
\text { magnetic Prandtl number } P_{m}=\frac{v_{i}}{\lambda_{i}},
\end{gathered}
$$

where the " $i$ " subscript represents values at the inner boundary. 


\subsubsection{Variable properties}

Recent $a b$ initio calculations suggest that electrical conductivity decreases with radius by several orders of magnitude in the outer regions of Jupiter-like objects (French et al. 2012). Low-mass stars, brown dwarfs and massive extrasolar planets will probably show a similar variation in electrical conductivity. An electrical conductivity profile approximately constant in the deep interior and exponentially decaying in outer portions represents a good model for giant planets (French et al. 2012). We model electrical conductivity normalized by its value at the inner boundary as (Gómez-Pérez et al. 2010, Duarte et al. 2013)

$$
\tilde{\sigma}(r)=\left\{\begin{array}{ll}
1+\left(\tilde{\sigma}_{m}-1\right)\left(\frac{r-r_{i}}{r_{m}-r_{i}}\right)^{a} & r<r_{m}, \\
\tilde{\sigma}_{m} e^{a\left(\frac{r-r_{m}}{r_{m}-r_{i}}\right) \frac{\tilde{\sigma}_{m}-1}{\tilde{\sigma}_{m}}} & r \geq r_{m}
\end{array} .\right.
$$

The electrical conductivity follows a polynomial in $r_{i}<r<r_{m}$ and decreases to $\tilde{\sigma}\left(r_{m}\right)=$ $\tilde{\sigma}_{m}$ (usually 0.5) near $r_{m}$. The exponential decay starts for $r \geq r_{m}$. The constant $a$ defines the rate of decay; $a$ is mostly equal to 9, except of two cases where it is 1 and 25 . The relative transition radius $\chi_{m}=r_{m} / r_{i}$ is $0.7,0.8,0.9$, or 0.95 . The magnetic diffusivity $\lambda=(\mu \tilde{\sigma}(r))^{-1}$ accordingly rises along the radius. We use such profiles in many of our anelastic dynamo simulations.

We also simulate cases with diffusivities, viz. viscosity, thermal diffusivity, magnetic diffusivity, varying as a function of density:

$$
(v, \kappa, \lambda)=(v, \kappa, \lambda)_{i} \sqrt{\frac{\tilde{\rho}_{i}}{\tilde{\rho}(r)}} .
$$

Such density dependence is similar to that used in many stellar dynamo simulations (e.g. Brun et al. (2004), Browning (2008)).

\subsubsection{Boundary conditions}

The mechanical boundary condition is either stress-free on both boundaries or rigid on the inner and stress-free on the outer boundary. On both boundaries constant entropy is imposed. We do not simulate cases with flux boundary conditions. For Boussinesq cases, no difference in scaling laws had been found between fixed temperature and fixed flux conditions (Aubert et al. 2009, Christensen 2010). The inner core is either conducting or insulating. The magnetic field matches a diffusive solution at the inner boundary in case of a conducting inner core and a potential field in case of an insulating inner core, while it always matches a potential field on the outer boundary.

\subsubsection{Numerical technique}

The anelastic equations (Eqs. 3.3-3.10) are time advanced using MagIC (Wicht 2002, Gastine and Wicht 2012). The anelastic version of MagIC has been benchmarked with recent community-based benchmark simulations (Jones et al. 2011). The mass-flux and 
the magnetic field are decomposed into poloidal an toroidal parts as

$$
\begin{aligned}
\tilde{\rho} \mathbf{u} & =\nabla \times(\nabla \times W \hat{r})+\nabla \times X \hat{r}, \\
\mathbf{B} & =\nabla \times(\nabla \times Y \hat{r})+\nabla \times Z \hat{r}
\end{aligned}
$$

where $W$ and $X(Y$ and $Z$ ) are poloidal and toroidal scalar potentials for mass-flux (magnetic field). The scalar potentials, along with $p$ and $s$, are then expanded using spherical harmonic functions in the azimuthal and the latitudinal direction. The radial expansion is done using Chebyshev polynomials. The equations are time-stepped by advancing nonlinear and Coriolis terms using an explicit second-order Adams-Bashforth scheme while the remaining terms are time-advanced using an implicit Crank-Nicolson algorithm (see Glatzmaier (1984) and Christensen and Wicht (2007) for more details).

\subsection{Results}

\subsubsection{Diagnostic parameters}

In the following discussions we will use several non-dimensional diagnostic parameters which are derived from the numerical simulations. They describe representative mean values of the flow velocity (or kinetic energy) and magnetic field strength (or magnetic energy). While in the Boussinesq case these properties have similar amplitude throughout the shell, with density stratification their magnitude can vary significantly with radius, in particular for the velocity. Hence the question arises which property to average in which way. We found that averaging energies divided by unit mass gives the best results.

The non-dimensional rms velocity is given by the Rossby number

$$
R o=\sqrt{\frac{1}{V} \int(\mathbf{u} \cdot \mathbf{u}) d V}
$$

where $V$ is volume of the fluid shell and the volume integral is evaluated in the fluid shell. The non-dimensional kinetic energy per unit mass is then $R o^{2} / 2$. The Lorentz number $L o$ describes the non-dimensional magnetic field strength and is defined as

$$
L o=\sqrt{\frac{\int(\mathbf{B} \cdot \mathbf{B}) d V}{\int \tilde{\rho} d V}}
$$

with $E_{m}=L o^{2} / 2$ being the non-dimensional magnetic energy per unit mass. The nondimensional power generated per unit mass by thermal buoyancy, scaled by $\Omega^{3} D^{2}$, is

$$
P=\frac{R a E^{2}}{P_{r}} \frac{\int u_{r} \tilde{\rho} g s d V}{\int \tilde{\rho} d V},
$$

where $u_{r}$ is the radial component of $\mathbf{u}$. The Nusselt number $N u$ is the ratio of total transported heat flux to the conducted heat flux. The rate of energy dissipated per unit mass by ohmic dissipation is

$$
D_{\lambda}=\frac{E}{P_{m}} \frac{\int \tilde{\lambda}(\nabla \times \mathbf{B})^{2} d V}{\int \tilde{\rho} d V}
$$


The ohmic fraction

$$
f_{\text {ohm }}=D_{\lambda} / P
$$

is the fraction of energy dissipated by joule dissipation alone. The characteristic time scale of magnetic energy dissipation is

$$
\tau_{m a g}=\frac{E_{m}}{D_{\lambda}}
$$

All diagnostic quantities are time-averaged after a statistically stationary state has been reached in the simulations. It would have been more consistent if in Eqs. (3.14-3.16) the magnetic energy, power, and dissipation would have been mass-normalized before integration (as is done in the case of the kinetic energy) instead of normalizing the total energy by the integral of $\tilde{\rho}$ (which is the non-dimensional total mass). However, the integrations have been done during the simulation and could not be repeated in modified form without repeating the whole run. Because the magnitude of these properties seems to vary less with radius than that of velocity, the difference is probably not critical.

We separate the dynamos resulting from our numerical simulation in two categories: the dipolar category contains dynamos with a dominant axisymmetric magnetic dipole (spherical harmonic degree $\ell=1$ and harmonic order $m=0$ ); the multipolar class contains all other kinds of dynamos. To carry out this separation we use the dipolarity $f_{d i p}$ which is the magnetic energy in the axisymmetric dipole normalized by the cumulated magnetic energy in harmonic degrees up to 12 , both evaluated at the outer boundary. Dynamos with $f_{\text {dip }}>0.3$ are considered dipolar. It must be noted that our data spans the range $10^{-4}<f_{\text {dip }}<0.9$ quite uniformly, hence a cutoff of 0.3 remains somewhat arbitrary. We justify our choice of cutoff in Sec. 3.3.4.

\subsubsection{Dynamo database}

Important physical attributes of various numerical setups used in this study are tabulated in Tab. 3.1. The database incorporates many important features, such as density stratification and variable transport properties. The largest density contrast in our simulation is $N_{\rho}=5.5$, i.e. $\rho_{i} / \rho_{o} \approx 245$. The aspect ratio $\eta$ is varied to cover dynamos operating in thick shells or nearly full spheres as well as dynamos in thinner shells (from $\eta=0.1$ to $\eta=0.75$ ). The polytropic index $m$ is also changed in a few cases to model different polytropic states. With these features our simulations seek to represent, in a simplified way, the dynamo action in the fully or partially convective and density stratified interiors in different classes of objects ranging from giant planets to low-mass stars.

In total, 273 dynamo cases are simulated. To the best of our knowledge this is the largest database of this kind. A portion of this database has already been used in earlier studies to explore different aspects of dynamo mechanism: A2a, B1a, A5 from Gastine et al. (2012) and Gastine et al. (2013); A2b, B1b from Duarte et al. (2013); A3a from Yadav et al. (2013). The full database can be found in the online supplementary table. More details, e.g. on the choice of model parameters, can be found in the references mentioned before. The simulations cover a large parameter space: $0.3 \leq P_{r} \leq 10,0.2 \leq$ $P_{m} \leq 20,1 \times 10^{-6} \leq E \leq 1 \times 10^{-3}$, and $2.5 \times 10^{5} \leq R a \leq 2.5 \times 10^{9}$. Except for two lowest 


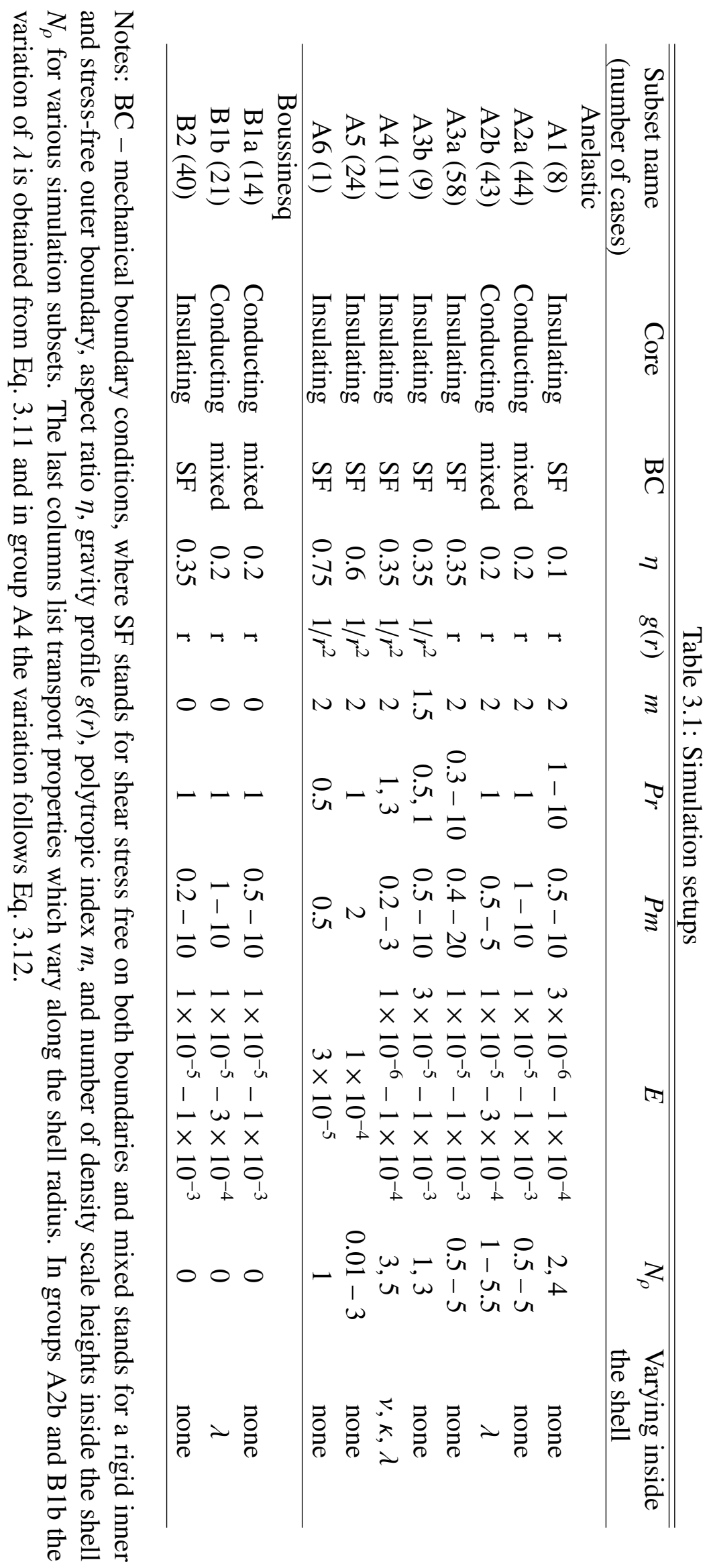


Ekman number simulations, all simulations were run until the simulated time was at least one magnetic diffusion time $\left(D^{2} / \lambda\right)$ and a statistically stationary temporal behavior was acquired. Depending on $\operatorname{Pr}$ and $P m$ viscous $\left(D^{2} / v\right)$ and thermal $\left(D^{2} / \kappa\right)$ diffusion time scales could be larger than the magnetic diffusion time, but because the magnetic field tends to equilibrate more slowly than the thermal or the velocity field, the latter time scale is more relevant. The range of our data-set is dictated by the computational need of a simulation. For example, low $E$ simulations are computationally demanding, but such dynamos can be obtained at lower $P m$ and hence as $E$ decreases so do our explored $P m$ values. No azimuthal symmetry was imposed in the simulations, except for the large aspect ratio run of A6 where two fold symmetry in longitude was used.

Our aim is to explore scaling laws in the rotationally dominated dynamic regime, i.e. Coriolis forces are dominant and inertia plays a secondary role, hence we report and analyze dynamos with $1 \times 10^{-6} \leq E \leq 1 \times 10^{-3}$ and avoid convection with very high supercritical Rayleigh numbers. Furthermore, to ensure fully developed convection in the spherical shell we use only dynamos with $N u \geq 2$.

\subsubsection{Velocity power scaling}

Scaling laws for velocity and magnetic field in Boussinesq models have been conventionally expressed in terms of flux-based Rayleigh number $R a_{Q}^{*}=R a(N u-1) E^{3} / P r$ which serves as a proxy for non-dimensional power $P$ (Christensen 2002, Christensen and Aubert 2006, Olson and Christensen 2006, Takahashi et al. 2008, Schrinner et al. 2012, Yadav et al. 2013, Schrinner 2013). $R a_{Q}^{*}$ is in general directly proportional to $P$ but the proportionality constant is determined by the particular simulations setup. In Boussinesq models the proportionality constant can be calculated in terms of shell aspect ratio and the distribution of buoynacy sources and sinks (Christensen and Aubert 2006, Aubert et al. 2009), but for our anelastic models its dependence on density stratification and variable transport coefficients becomes nontrivial. Following Kaspi et al. (2009), Gastine and Wicht (2012) expressed the velocity scaling in their anelastic convection simulations using a mass averaged $\left\langle R a_{Q}^{*}\right\rangle_{\rho}$, where $\langle\cdot\rangle_{\rho}$ stands for $\int(\cdot) \tilde{\rho} r^{2} d r / \int \tilde{\rho} r^{2} d r$, to better collapse simulations with different density stratifications. However, different scaling laws in our database, which includes cases with radially varying transport coefficients, do not collapse well with $\left\langle R a_{Q}^{*}\right\rangle_{\rho}$ as a control parameter. We find that the power $P$, whose time-average value is calculated explicitly in each simulation, is a much better control parameter for collapsing diverse simulation setups.

Least-square-optimization of the Rossby number $R o$ as a function of power $P$ and magnetic Prandtl number $P_{m}$ for our database results in

$$
R o=2.47 \frac{P^{0.45}}{P_{m}^{0.13}} .
$$

This empirical scaling is represented by a solid line in Fig. 3.1. The dynamo cases marked by a "+" in Fig. 3.1 (subset A2b and B1b; see Tab. 3.1) are excluded from the fit. The excluded cases have exponentially decaying electrical conductivity in the outer parts of the spherical shell. In these regions Lorentz forces are weak which allows for the development of strong zonal flows that enhance the rms velocity (Duarte et al. 2013). This is 
evident in Fig. 3.1 where most of these cases have higher $R o$ and lie above other dynamo cases. The dynamo cases from subset A4 with moderate conductivity variation, which are marked by a black dot in Fig. 3.1, do not show enhanced zonal flow and their Rossby numbers agree well with that of the other dynamos.

The scatter of data points in Fig. 3.1 is primarily due to differences in the relative strength of zonal flows (Yadav et al. 2013). Magnetic field geometry, which can vary substantially from one simulation to another, affects the zonal flow through Maxwell stresses (Browning 2008). Yadav et al. (2013) reported two different Ro scalings for dipolar and multipolar dynamos (subset A3a; see Tab. 3.1), although the difference was relatively minor. They argued that this offset is due to more efficient zonal flow quenching by magnetic braking in dipolar dynamos as compared to the multipolar ones. Such consistent dichotomy is washed out in Fig. 3.1, probably due to our much more diverse dataset.

On similar lines as Yadav et al. (2013), we define a convective Rossby number $R o_{\text {conv }}$ which is calculated by excluding zonal and meridional flow components. A best-fit of $R o_{\text {conv }}$ gives

$$
R o_{c o n v}=1.65 \frac{P^{0.42}}{P_{m}^{0.08}},
$$

which is portrayed in Fig. 3.2. As compared to Fig. 3.1 the scatter in Fig. 3.2 is much smaller and also cases with a poorly conducting exterior region are now well fitted. On the other hand, the Rossby number based on the zonal flow component is strongly scattered in our database (not shown) and does not exhibit a consistent scaling, in contrast to what has been found in purely hydrodynamic spherical shell convection (Aubert et al. 2001, Christensen 2002, Aubert 2005, Showman et al. 2011, Gastine and Wicht 2012). As discussed above, this is due to the variable influence of magnetic field on the zonal flow via Maxwell stresses.

A scaling law of the type $R o \propto P^{\alpha}$ with $0.41 \leq \alpha \leq 0.44$ has been reported by many studies of Boussinesq numerical dynamo simulations: in a wide control parameter study with rigid mechanical boundaries (Christensen and Aubert 2006, Takahashi et al. 2008); for compositional and thermal convection with variable core size, including cases with nearly fully convective interiors (Aubert et al. 2009); in simulations with different thermal boundary conditions (Christensen 2010); and for cases with stress-free mechanical boundaries (Yadav et al. 2013).

The theoretical justifications for the velocity scaling $R o \propto P^{\alpha}$, with $\alpha$ slightly larger than 0.4, is not entirely clear. Christensen (2010) discussed several proposed scaling approaches and showed that they all lead to a law of this form, but with different exponents. The mixing length theory (Böhm-Vitense 1958) applied to planetary interiors predicts $\alpha=1 / 3$, but is probably not applicable to rapidly rotating systems (Christensen 2010). Aubert et al. (2001) considered a triple force balance of Coriolis, inertial, and Archimedean forces (the so-called CIA balance) in the hydrodynamic fluid vorticity equation and derived $\alpha=2 / 5$. Starchenko and Jones (2002) obtained $\alpha=1 / 2$ based on a balance of Coriolis and buoyancy forces. The prediction of the CIA balance is the closest to the exponent found by fitting the data from dynamo simulations, but underpredicts it slightly. Davidson (2013) presented a scaling theory starting from the assumption that the magnetic field is independent of rotation rate and scales with the cubic root of the power, 


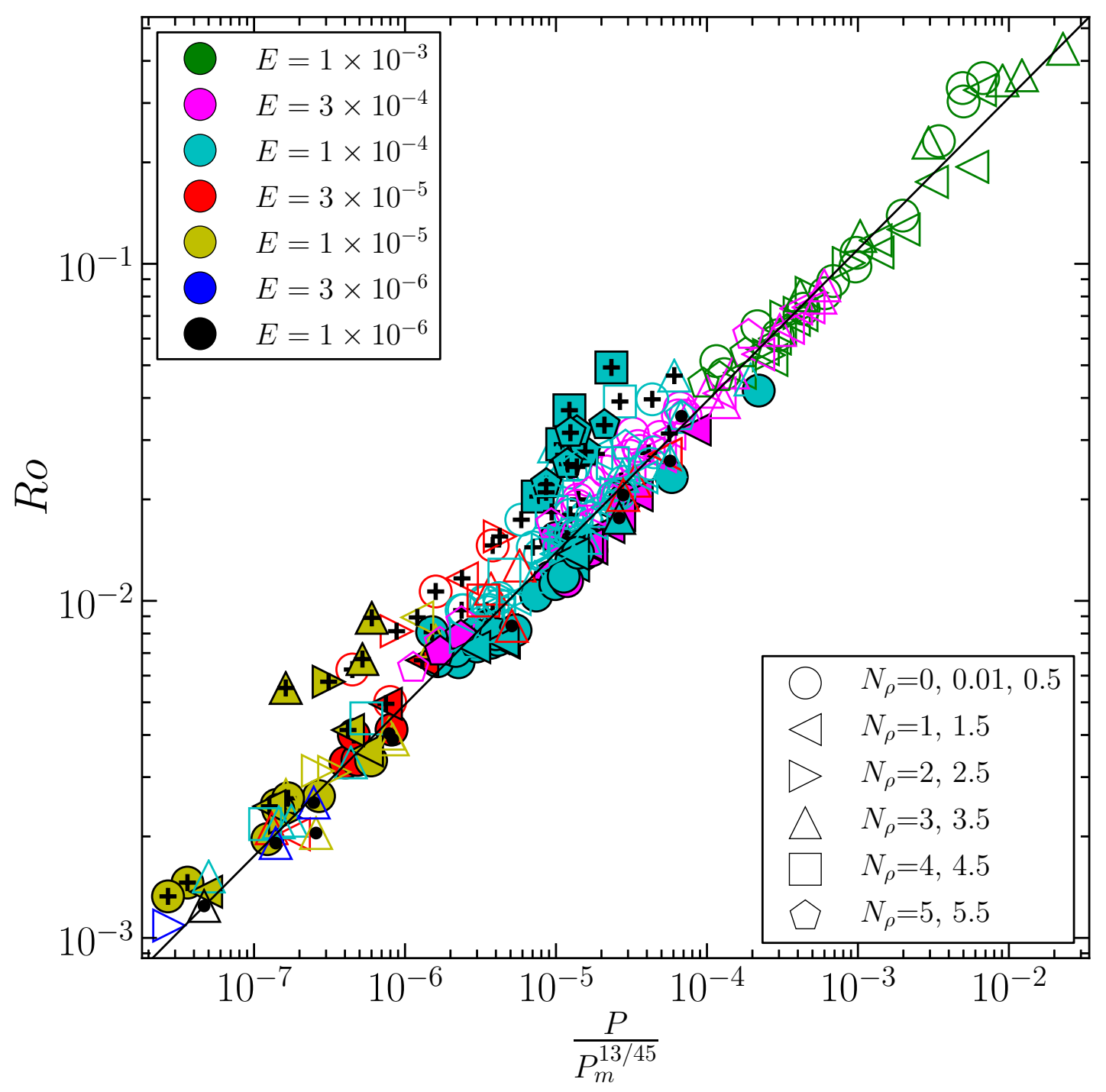

Figure 3.1: Rossby number versus a combination of non-dimensional power and magnetic Prandtl number. The solid line represents $R o=2.47 P^{0.45} P_{m}{ }^{-0.13}$. Filled (empty) symbols display dipolar (multipolar) dynamos. The Ekman number is color coded and the symbol shape indicates degree of density stratification. Symbols containing a "+" marker have exponentially decaying electrical conductivity in the outer regions (subset A2b and B1b in Tab. 3.1) and the ones carrying a dot symbol have moderate conductivity variations (subset A4 in Tab. 3.1). 


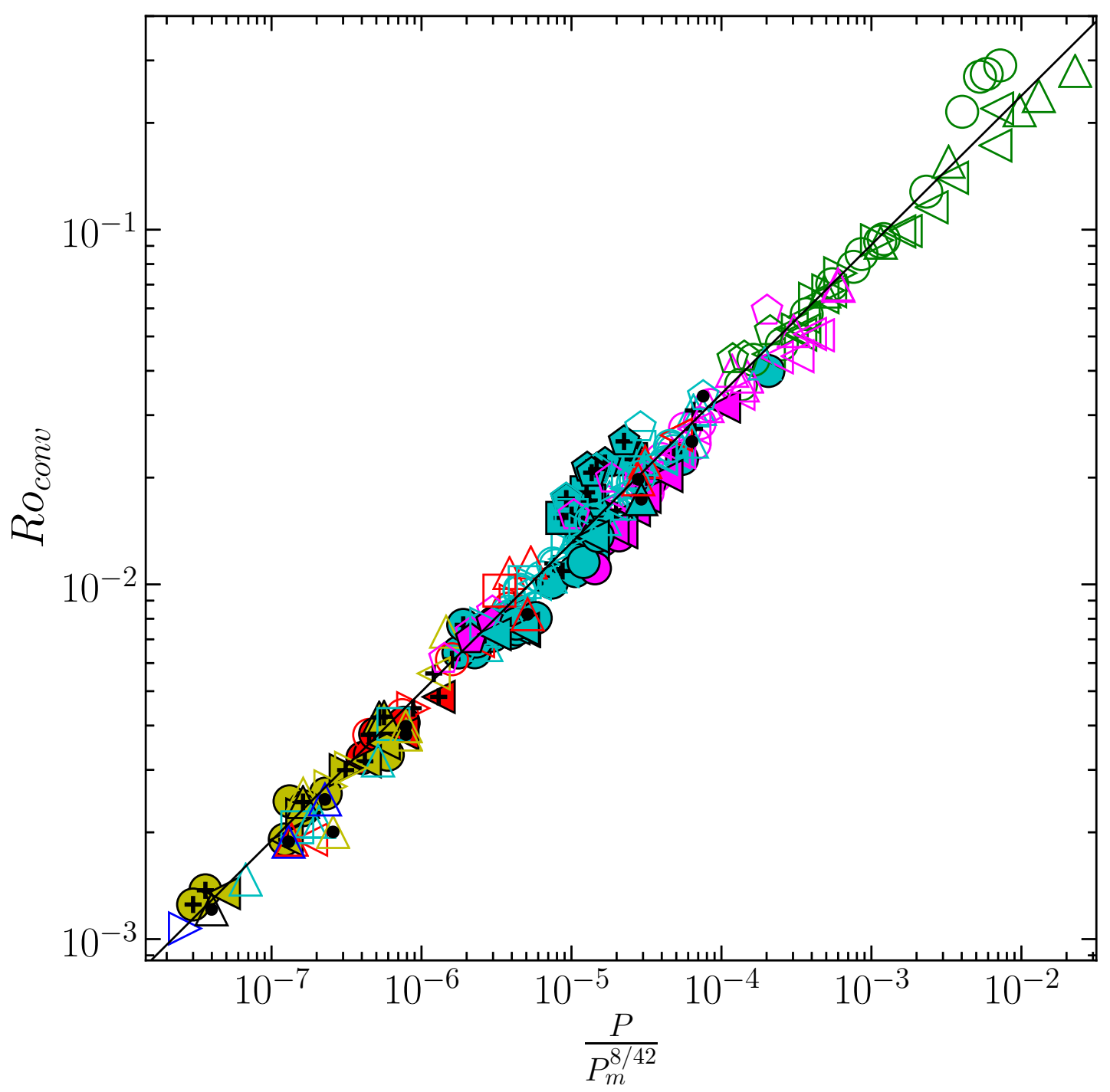

Figure 3.2: Convective Rossby number versus a combination of power and magnetic Prandtl number. The solid line represents $R o_{c o n v}=1.65 P^{0.42} P_{m}{ }^{-0.08}$. Refer to Fig. 3.1 for symbol description. 
as confirmed by the numerical results (see next section). Davidson (2013) considers a triple force balance of Lorentz, Archimedean, and Coriolis forces (the so called MAC balance) to then derive a velocity scaling of the form $R o \propto P^{4 / 9}$. The exponent is very close to our empirically obtained exponents $\alpha=0.45$ (Eq. 3.19) or $\alpha=0.42$ (Eq. 3.20). None of the scaling theories for velocity proposed so far accounts for the $P_{m}$ dependence. Inclusion of $P_{m}$ as a fit parameter significantly reduces the scatter of data-points (Christensen and Aubert 2006, Yadav et al. 2013, Stelzer and Jackson 2013).

\subsubsection{Magnetic field scaling}

In the Boussinesq dynamo simulations, Christensen (2010), Schrinner et al. (2012), and Yadav et al. (2013) found that the magnetic field strength obeys very similar scaling laws for dipolar and for multipolar dynamos, except for a difference in the pre-exponential constant. Therefore we treat the two classes of dynamos separately. A least-square fit of the Lorentz number corrected by $f_{o h m}$ as a function of $P$ and $P_{m}$ leads to

$$
\frac{L o}{\sqrt{f_{\text {ohm }}}}=1.08 P^{0.35} P_{m}^{0.13}
$$

for the dipolar dynamos and

$$
\frac{L o}{\sqrt{f_{\text {ohm }}}}=0.78 P^{0.34} P_{m}^{0.09}
$$

for the multipolar ones. These two shifted scalings provide a basis for separating dynamos in dipolar and multipolar categories. We found that $f_{\text {dip }}=0.3$ provides a reasonably good cutoff; lower or higher cutoffs start to mix data points and we loose the demarcation noticeable in Fig. 3.3.

The two expressions (3.21) and (3.22) are very similar except for the larger prefactor in the dipolar scaling. We therefore attempt to fit both classes of dynamos with the same approximate exponents for $P$ and $P_{m}$

$$
\frac{L o}{\sqrt{f_{\text {ohm }}}} \approx c P^{\frac{1}{3}} P_{m^{\frac{1}{10}}}
$$

where $\mathrm{c}$ is fitted separately for dipolar and for multipolar dynamos, giving 0.9 and 0.7 , respectively. This approximate scaling is plotted in Fig. 3.3. Yadav et al. (2013) argued that the higher field strengths in dipolar dynamos can be attributed to lower flow velocity in dipolar dynamos (lack of strong zonal flows). Such argument may not work for our database since both dipolar and multipolar dynamos are consistent with a single velocity scaling (see previous section). Further study is required to explain this behaviour.

Two different theoretical approaches have been put forward to explain the scaling of the Lorentz number with (approximately) the cubic root of the power. Christensen and Aubert (2006) take a velocity scaling $R o \propto P^{0.4}$ as given and use the results of Christensen and Tilgner (2004) that the magnetic dissipation time scale $\tau_{\text {mag }}$ (in rotational units) is approximately proportional to $R o^{-1}$. In a different approach, Davidson (2013) uses the finding by Kunnen et al. (2010) that the small scale vorticity in rotating convection is independent of rotation rate. Assuming that this also holds for vorticity at the magnetic 


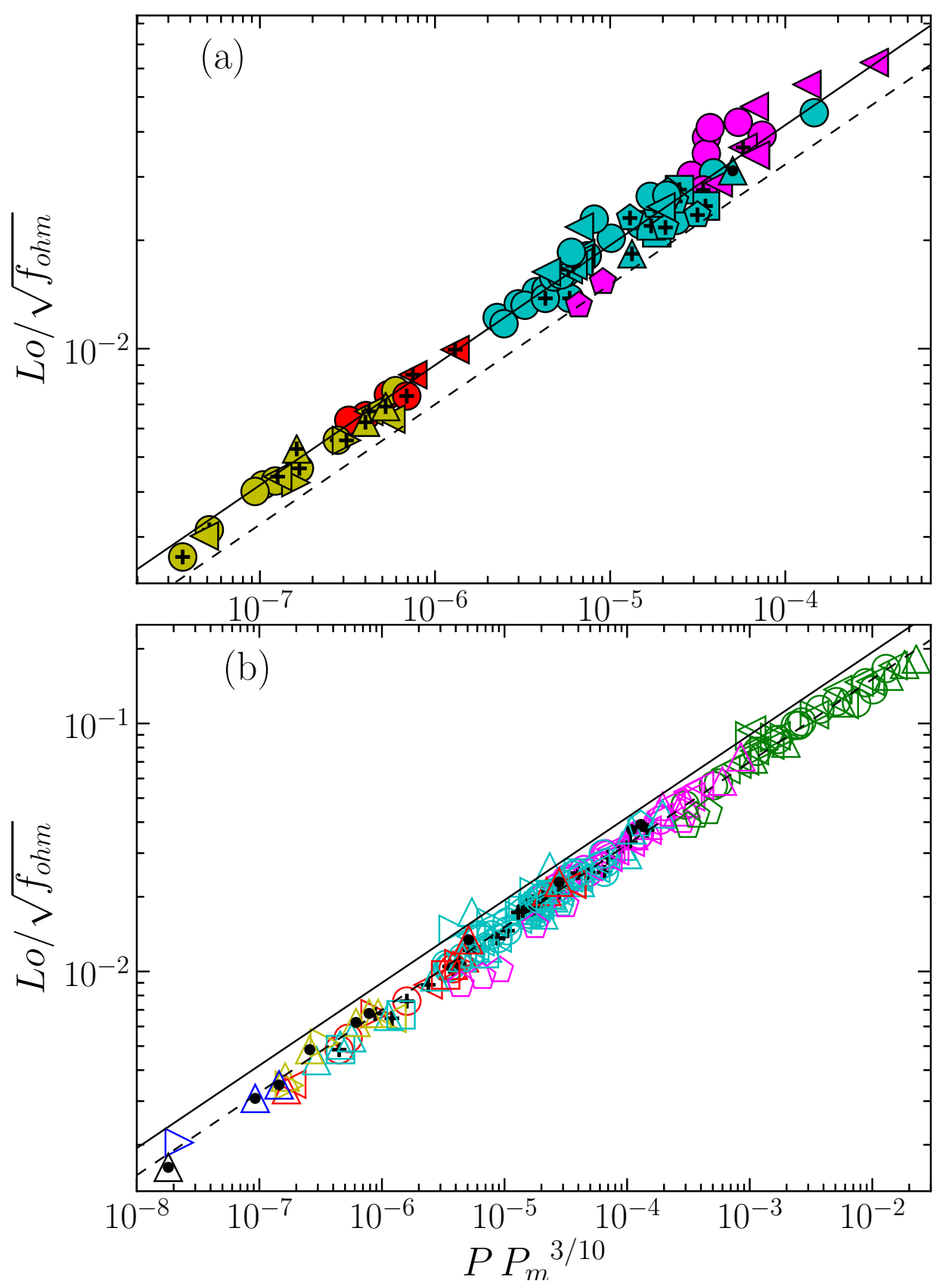

Figure 3.3: Lorentz number corrected for fraction of ohmic dissipation versus a combination of non-dimensional power and magnetic Prandtl number. (a) dipolar dynamos, the fit by the solid line follows Eq. (3.23) with $c=0.9$. (b) multipolar dynamos, the fit by the broken line is for $c=0.7$. The two fitting lines are replicated in both plots to show the offset. Refer to Fig. 3.1 for symbol description. 
dissipation length scale $l_{\min }$, for which the local (scale-dependent) magnetic Reynolds number is of order one, he infers that also $l_{\text {min }}^{2} / \lambda \propto \tau_{\text {mag }}$ must be independent of the rotation rate. Setting the $f_{\text {ohm }}$ factor aside, the magnetic energy is the product of power and magnetic dissipation time and is therefore also independent of the rotation rate. If magnetic field is only a function of power, dimensional arguments dictate that it must depend on the cubic root of the power.

Davidson's scaling theory predicts a dependence of the magnetic diffusion time on the Rossby number as $\tau_{\text {mag }} \propto R o^{-3 / 4}$, significantly weaker than the $R o^{-1}$ dependence originally envisaged by Christensen and Tilgner (2004). The scaling of $\tau_{\text {mag }}$ vs. Ro in numerical dynamo models had been revisited by Christensen (2010), Yadav et al. (2013), and Stelzer and Jackson (2013) who all found exponents somewhat weaker than -1. In Fig. 3.4 we plot $\tau_{m a g}$ for dipolar and multipolar dynamos against $R o$. The scatter is larger, especially for dipolar dynamos, than in the cases of fitting Ro and Lo. A best-fit line for the dipolar dynamos is

$$
\tau_{\text {mag }}=1.51 R o^{-0.63}
$$

and for multipolar dynamos it is

$$
\tau_{\text {mag }}=0.67 R o^{-0.69} .
$$

This shows that the scaling exponent may be even slighly weaker than the recent prediction by Davidson (2013).

\subsection{Discussion and conclusions}

In this study we analyze a large number of numerical simulations of dynamo in rapidly rotating spherical shells to infer empirical scaling laws for mean velocity and mean magnetic field. Our database contains anelastic compressible dynamos with different shell thicknesses, different density stratifications with density contrast upto $\approx 245$, and radially varying transport properties (viscosity, thermal diffusivity, magnetic diffusivity). These models capture, in a simplified way, conditions that are relevant for rapidly rotating gas planets and low-mass stars.

Our study shows that it is possible to relate flow velocity and magnetic field strength to the available power, by a single scaling law in each case, for a wide range of different conditions. In particular the same scaling law covers incompressible cases and cases with strong density stratification. Our finding sanctifies the application of the same magnetic field scaling by Christensen et al. (2009) to such diverse objects as the Earth's core and low-mass stars.

Our best-fitting power exponent of 0.45 for the Rossby number, as a measure of the characteristic velocity, is very close to the value of 4/9 obtained by the scaling theory of Davidson (2013) which assumes a MAC balance. Our data are better collapsed when the Rossby number is calculated only with the convective flow (excluding the zonal and meridional flow component) and the optimal exponent of 0.42 is intermediate between Davidson's prediction and the value of 2/5 inferred from a CIA balance (Aubert et al. 2001). Because our simulations may not have exactly reached an asymptotic regime, the precise value of the exponent and its theoretical justification remain somewhat uncertain. 

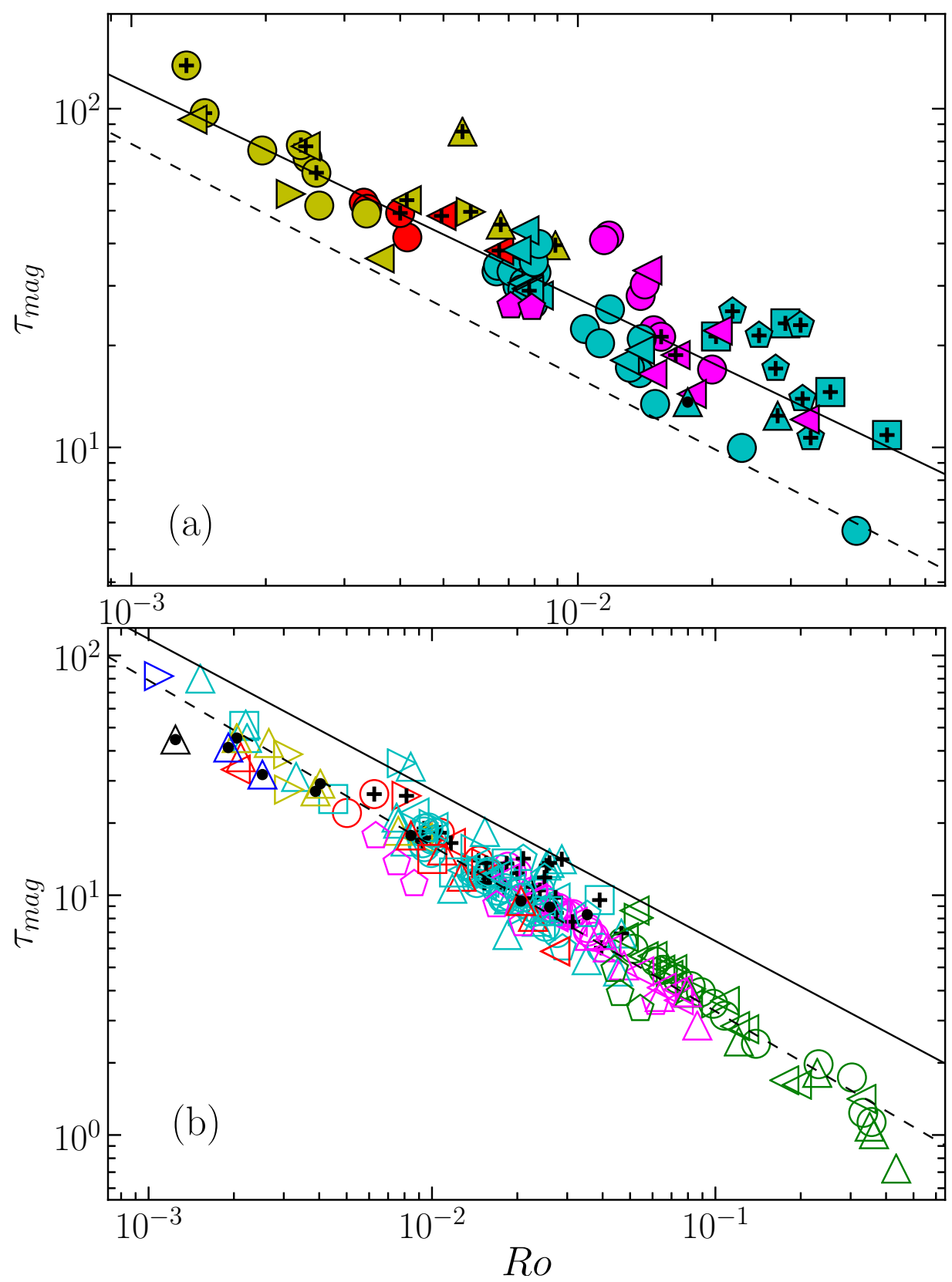

Figure 3.4: Magnetic dissipation time versus Rossby number. (a) dipolar dynamos, (b) multipolar dynamos. The solid and broken lines for the best fits to the two subsets (Eq. (3.24) and (3.25)) are shown in both panels. Refer to Fig. 3.1 for symbol description. 
Our data for the Lorentz number which measures magnetic field strength are fully compatible with a cubic root dependence on the non-dimensional power (after correcting for that part of the energy flux that does not contribute to magnetic field generation). This makes the scaling independent of the rotation rate, which is a central point in the scaling theory of Davidson (2013). While both dipolar and multipolar dynamos follow basically the same scaling law, we find that in the dipolar case the field strength is larger by $\approx 30 \%$. In Yadav et al. (2013) we attributed this difference to a slightly weaker flow velocity in dipolar dynamos, but in our present study we do not find a significant difference in the Rossby number between dipolar and multipolar cases. However, we note that the ohmic dissipation time is systematically shorter for multipolar dynamos (open symbols in Fig. 3.4) than it is for dipolar dynamos (filled symbols), i.e., the generated magnetic energy is more rapidly dissipated in the multipolar case, leading to a lower mean field strength. The reason for the longer dissipation time of dipolar dynamos could be that more magnetic energy is stored in large spatial scales where it is less vulnerable to dissipative destruction.

Although we find that Boussinesq and anelastic dynamos are described by the same scaling laws, it must be noted that qualitative differences exist between the two. As compared to the Boussinesq models, flow velocity in anelastic models is faster and has smaller length scales in the outer parts of the fluid shell as compared to the deeper interior (Browning 2008, Gastine and Wicht 2012). This difference affects the nature of the resulting dynamo. For instance, Gastine et al. (2012) find that dipolar dynamos give away to multipolar ones as the density stratification is increased (keeping other parameters the same). They argue that separation of the flow length scales makes the flow structure in highly stratified cases similar to convection in thin shell geometries which prefer non-dipolar dynamos (Stanley et al. 2005). However, Duarte et al. (2013) show that models with variable electrical conductivity can push the dynamo generation to the deep interior and dipolar dynamos can be easily obtained for even highly stratified cases.

The mismatch between the control parameters of numerical simulations and the astrophysical objects is mainly caused by the expected extremely low values of the various transport coefficients in the latter. The scaling laws we present here are expressed in terms of non-dimensional buoyancy power per unit mass, $P$, which does not involve any diffusive parameters (barring the small $P_{m}$ dependence). Our simulations span $10^{-8} \lesssim P \lesssim$ $10^{-2}$. To put this range into perspective, we calculate $P$ for the rapidly rotating M4.5 star EV Lac using the parameters quoted in Christensen et al. (2009, Supplementary information). The resulting value, $P \approx 2 \times 10^{-8}$, lies within the range covered by our database. As shown by Christensen et al. (2009) the observed surface field strength roughly agrees with the power-based scaling law.

There are few outstanding issues which need further exploration. As has recently been emphasized by Stelzer and Jackson (2013), some dependence on the magnetic Prandtl number is required for an optimal fit of the dynamo simulation data. Although the influence of $P_{m}$ in the scaling laws is secondary, its value in natural dynamos is several orders of magnitude lower than it is in the simulations. Ignoring or including $P_{m}$ in the scaling laws makes a difference of nearly an order of magnitude in the prediction for planets or stars. Christensen and Tilgner (2004) argued that the $P_{m}$-dependence, although needed for fitting the model data at $P_{m} \approx 1$, would disappear in the astrophysically relevant range $P_{m} \ll 1$ and Christensen and Aubert (2006) found good agreement between prediction 
and observation in case of the Earth's dynamo only when the dependence on the magnetic Prandtl number is ignored. However, the assumption that the $P_{m}$-dependence vanishes at small values has not yet been confirmed by theory or by numerical simulations, which are not available for $P_{m}$ much smaller than one.

Another issue is the independence of magnetic field scaling from rotation rate that is implied by our scaling law. For low-mass stars a saturated (rotation-rate independent) magnetic surface flux is observed for very rapid rotators with low Rossby number, but for larger values of the Rossby number it decreases roughly proportionally with decreasing rotation rate (e.g. Reiners et al. 2009). Schrinner (2013) argues that the rotational dependence at large Rossby number may enter through its influence on the ohmic dissipation factor $f_{\text {ohm }}$, challenging the often made assumption that $f_{\text {ohm }} \approx 1$ in all kinds of natural dynamos.

One problem with applying our scaling laws to stars and planets is that they predict average values inside the dynamo, whereas observations usually relate to the strength of the magnetic field or part of the field at the surface of the object. The assumption that the two values can be related by a fixed factor is probably too simplistic. It is conceivable that a dependence of this factor on the rotation rate could contribute to the observed dependence of the surface magnetic flux on the Rossby number in M-stars with moderate to low rotation rates.

Our work extends earlier studies (Christensen and Aubert 2006, Takahashi et al. 2008, Aubert et al. 2009, Christensen 2010, Schrinner et al. 2012, Yadav et al. 2013). It shows that previously found scaling laws for velocity and magnetic field strength in rotating dynamos also apply when strong radial variations in density or transport properties are present, as expected in the conducting cores of gas planets, brown dwarfs and low-mass stars. This strongly supports the notion that the power-based scaling laws are rather universal. For the magnetic field scaling there is a decent agreement with observation for several solar system planets and certain classes of stars (Christensen et al. 2009, Christensen 2010). However, the velocity scaling has not yet been tested against observation (except for Earth's core). This together with the open questions on the role of the magnetic Prandtl number, the cause for weaker surface magnetic fields at less rapidly rotating stars, and the relation between internal and surface magnetic fields keep this research area vibrant and provide an exciting avenue for future explorations.

\section{Acknowledgements}

We thank the anonymous referee for helping to improve the presentation of our paper. We acknowledge funding from the Deutsche Forschungsgemeinschaft (DFG) through Project SFB 963 / A17 and through the special priority program 1488 (PlanetMag, http: //www . planetmag. de) of the DFG. Simulations were run on the GWDG computational facility, Göttingen and the Regionales Rechenzentrum für Niedersachsen (RRZN) at Leibniz University Hannover operating under the Norddeutscher Verbund zur Förderung des Hoch- und Höchstleistungsrechnens (HLRN). All the figures were generated using matplotlib (www.matplotlib.org). 


\section{Bibliography}

Aubert, J. (2005). Steady zonal flows in spherical shell dynamos. J. Fluid Mech., 542(1), 53-67.

Aubert, J., Brito, D., Nataf, H.-C., Cardin, P., and Masson, J.-P. (2001). A systematic experimental study of rapidly rotating spherical convection in water and liquid gallium. Phys. Earth Planet. Int., 128(1-4), 51-74.

Aubert, J., Labrosse, S., and Poitou, C. (2009). Modelling the palaeo-evolution of the geodynamo. Geophys. J. Int., 179(3), 1414-1428.

Böhm-Vitense, E. (1958). Über die Wasserstoffkonvektionszone in Sternen verschiedener Effektivtemperaturen und Leuchtkräfte. Mit 5 Textabbildungen. ZAp, 46, 108.

Braginsky, S. I. and Roberts, P. H. (1995). Equations governing convection in earth's core and the geodynamo. Geophys. Astrophys. Fluid Dyn., 79(1-4), 1-97.

Browning, M. K. (2008). Simulations of dynamo action in fully convective stars. ApJ, 676(2), 1262-1280.

Brun, A. S., Miesch, M. S., and Toomre, J. (2004). Global-scale turbulent convection and magnetic dynamo action in the solar envelope. ApJ, 614(2), 1073.

Christensen, U. and Wicht, J. (2007). Numerical dynamo simulations. In G. Schubert, editor, Treatise of Geophysics, Vol. 8. Elsevier, Amsterdam.

Christensen, U. R. (2002). Zonal flow driven by strongly supercritical convection in rotating spherical shells. J. Fluid Mech., 470, 115-133.

Christensen, U. R. (2010). Dynamo scaling laws and applications to the planets. Space Sci. Rev., 152, 565-590.

Christensen, U. R. and Aubert, J. (2006). Scaling properties of convection-driven dynamos in rotating spherical shells and application to planetary magnetic fields. Geophys. J. Int., 166(1), 97-114.

Christensen, U. R. and Tilgner, A. (2004). Power requirement of the geodynamo from ohmic losses in numerical and laboratory dynamos. Nature, 429, 169-171.

Christensen, U. R., Holzwarth, V., and Reiners, A. (2009). Energy flux determines magnetic field strength of planets and stars. Nature, 457, 167. 
Curtis, S. A. and Ness, N. F. (1986). Magnetostrophic balance in planetary dynamos: Predictions for neptune's magnetosphere. J. Geophys. Res., 91(A10), 11003-11008.

Davidson, P. A. (2013). Scaling laws for planetary dynamos. Geophys. J. Int., 195(1), $67-74$.

Duarte, L. D., Gastine, T., and Wicht, J. (2013). Anelastic dynamo models with variable electrical conductivity: An application to gas giants. Phys. Earth Plan. Inter, 222(0), $22-34$.

French, M., Becker, A., Lorenzen, W., Nettelmann, N., Bethkenhagen, M., Wicht, J., and Redmer, R. (2012). Ab initio simulations for material properties along the jupiter adiabat. ApJS, $\mathbf{2 0 2}(1), 5$.

Gastine, T. and Wicht, J. (2012). Effects of compressibility on driving zonal flow in gas giants. Icarus, 219(1), $428-442$.

Gastine, T., Duarte, L., and Wicht, J. (2012). Dipolar versus multipolar dynamos: the influence of the background density stratification. $A \mathcal{E} A, \mathbf{5 4 6}, \mathrm{A} 19$.

Gastine, T., Morin, J., Duarte, L., Reiners, A., Christensen, U., and Wicht, J. (2013).

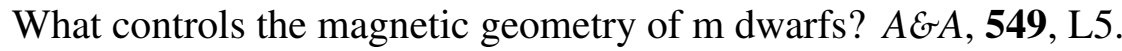

Gilman, P. A. and Glatzmaier, G. A. (1981). Compressible convection in a rotating spherical shell. i-anelastic equations. ApJS, 45, 335.

Glatzmaier, G. A. (1984). Numerical simulations of stellar convective dynamos. i. the model and method. J. Comp. Phys., 55(3), 461-484.

Gómez-Pérez, N., Heimpel, M., and Wicht, J. (2010). Effects of a radially varying electrical conductivity on $3 \mathrm{~d}$ numerical dynamos. Phys. Earth Planet. Int., 181(1), 42-53.

Jones, C., Boronski, P., Brun, A., Glatzmaier, G., Gastine, T., Miesch, M., and Wicht, J. (2011). Anelastic convection-driven dynamo benchmarks. Icarus, 216(1), 120-135.

Jones, C. A. (2011). Planetary magnetic fields and fluid dynamos. Annual Rev. of Fluid Mech., 43(1), 583-614.

Jones, C. A., Kuzanyan, K. M., and Mitchell, R. H. (2009). Linear theory of compressible convection in rapidly rotating spherical shells, using the anelastic approximation. $J$. Fluid Mech., 634, 291-319.

Kaspi, Y., Flierl, G. R., and Showman, A. P. (2009). The deep wind structure of the giant planets: Results from an anelastic general circulation model. Icarus, 202(2), 525-542.

Kunnen, R., Geurts, B., and Clercx, H. (2010). Experimental and numerical investigation of turbulent convection in a rotating cylinder. J. Fluid Mech., 642(1), 445-476.

Lantz, S. and Fan, Y. (1999). Anelastic magnetohydrodynamic equations for modeling solar and stellar convection zones. ApJS, 121(1), 247. 
Mizutani, H., Yamamoto, T., and Fujimura, A. (1992). A new scaling law of the planetary magnetic fields. Adv. Space Res., 12(8), 265 - 279.

Olson, P. and Christensen, U. R. (2006). Dipole moment scaling for convection-driven planetary dynamos. Earth and Planet. Sci. Lett., 250(3âĂŞ4), $561-571$.

Ossendrijver, M. (2003). The solar dynamo. AEA Rev., 11(4), 287-367.

Reiners, A., Basri, G., and Browning, M. (2009). Evidence for magnetic flux saturation in rapidly rotating $\mathrm{m}$ stars. $A p J, \mathbf{6 9 2}(1), 538$.

Sano, Y. (1993). The magnetic fields of the planets: A new scaling law of the dipole moments of the planetary magnetism. J. Geomagn. Geoelectr., 45(1), 65-77.

Schrinner, M. (2013). Rotational threshold in global numerical dynamo simulations. $M N$ RAS Let., 431(1), L78-L82.

Schrinner, M., Petitdemange, L., and Dormy, E. (2012). Dipole collapse and dynamo waves in global direct numerical simulations. ApJ, 752(2), 121.

Showman, A. P., Kaspi, Y., and Flierl, G. R. (2011). Scaling laws for convection and jet speeds in the giant planets. Icarus, 211(2), 1258-1273.

Stanley, S., Bloxham, J., Hutchison, W. E., and Zuber, M. T. (2005). Thin shell dynamo models consistent with MercuryâĂŹs weak observed magnetic field. Earth and Planet. Sci. Lett., 234, 27 - 38.

Starchenko, S. and Jones, C. (2002). Typical velocities and magnetic field strengths in planetary interiors. Icarus, 157(2), 426-435.

Stelzer, Z. and Jackson, A. (2013). Extracting scaling laws from numerical dynamo models. Geophys. J. Int., 193(3), 1265-1276.

Stevenson, D. J. (1979). Turbulent thermal convection in the presence of rotation and a magnetic field: A heuristic theory. Geophys. Astrophys. Fluid Dyn., 12(1), 139-169.

Takahashi, F., Matsushima, M., and Honkura, Y. (2008). Scale variability in convectiondriven MHD dynamos at low Ekman number. Phys. Earth Planet. Int., 167(3-4), 168178.

Wicht, J. (2002). Inner-core conductivity in numerical dynamo simulations. Phys. Earth Planet. Int., 132, 281-302.

Wicht, J. and Tilgner, A. (2010). Theory and modeling of planetary dynamos. Space Sci. Rev., 152(1), 501-542.

Yadav, R. K., Gastine, T., and Christensen, U. R. (2013). Scaling laws in spherical shell dynamos with free-slip boundaries. Icarus, 225(1), $185-193$. 


\title{
4 From solar-like to anti-solar differential rotation in cool stars
}

\begin{abstract}
Stellar differential rotation can be separated into two main regimes: solarlike when the equator rotates faster than the poles and anti-solar when the polar regions rotate faster than the equator. We investigate the transition between these two regimes with 3-D numerical simulations of rotating spherical shells. We conduct a systematic parameter study which also includes models from different research groups. We find that the direction of the differential rotation is governed by the contribution of the Coriolis force in the force balance, independently of the model setup (presence of a magnetic field, thickness of the convective layer, density stratification). Rapidly-rotating cases with a small Rossby number yield solar-like differential rotation, while weakly-rotating models sustain anti-solar differential rotation. Close to the transition, the two kinds of differential rotation are two possible bistable states. This study provides theoretical support for the existence of anti-solar differential rotation in cool stars with large Rossby numbers ${ }^{1,2}$.
\end{abstract}

\subsection{Introduction}

The solar surface rotates differentially with the equatorial regions rotating faster than the poles. In addition, helioseismic measurements revealed the internal rotation profile of the Sun: $(i)$ the outer convective region exhibits significant latitudinal gradients of shear; (ii) a strong radial differential rotation is observed at the bottom of the convective zone forming the tachocline; (iii) and the radiative core rotates nearly uniformly (e.g. Thompson et al. 2003).

In cool stars other than the Sun, the surface differential rotation can be inferred from different measurements techniques encompassing Doppler imaging (e.g. Collier Cameron et al. 2002), Fourier transform of the spectral lines (e.g. Reiners and Schmitt 2002) or period detection in the photometric measurements (e.g. Reinhold et al. 2013). The latitudinal differential rotation in stars is usually described by a single-parameter law of the form $\Omega(\theta)=\Omega_{e}\left(1-\alpha \sin ^{2} \theta\right), \theta$ being the latitude and $\Omega_{e}$ the angular velocity at the equator. Differential rotation is then usually categorised as "solar-like" when $\alpha>0\left(\alpha_{\odot}=0.2\right)$, or

\footnotetext{
${ }^{1}$ The content of this chapter has been reproduced from: T. Gastine, R. K. Yadav, J. Morin, A. Reiners, and J. Wicht, "From solar-like to anti-solar differential rotation in cool stars", MNRAS Let. 438, L76-L80, 2013. DOI: $10.1093 / \mathrm{mnrasl} / \mathrm{slt} 162$

${ }^{2}$ Contribution: I ran simulations to explore the effect of magnetic field on differential rotation. The data from these simulations was used to generate Figure 4.6. Thomas Gastine and I developed the ideas presented in Section 4.3.2 together.
} 
as "anti-solar" when the polar regions rotate faster than the equator (i.e. $\alpha<0$ ). For main and pre-main sequence stars, observations of absolute surface shear show some dependence on rotation period and effective temperature (Barnes et al. 2005, Ammler-von Eiff and Reiners 2012, Reinhold et al. 2013). Information on the sign of differential rotation is very sparse because observational signatures are very subtle (Fourier technique) or only the absolute value is obtained (photometric technique). Up to now, anti-solar differential rotation has only been reported for a handful of $\mathrm{K}$ giant stars observed with the Doppler imaging technique (e.g. Strassmeier et al. 2003, Weber et al. 2005, Kovári et al. 2007). We may thus wonder what determines the sign of differential rotation in cool stars?

The first theoretical approach to model stellar differential rotation relies on hydrodynamical mean-field models (e.g. Ruediger 1989). In a similar way as in the mean-field dynamo models, the velocity components $u_{i}$ are therefore decomposed into a mean-field contribution $\bar{u}_{i}$ and a fluctuating part $u_{i}^{\prime}$. The quadratic correlations of the fluctuating quantities, such as Reynolds stresses $Q_{i j}=\overline{u_{i}^{\prime} u_{j}^{\prime}}$, are then parametrised as functions of the mean-field quantities only. Reynolds stresses are for instance expanded assuming $Q_{i j}=\Lambda_{i j k} \bar{\Omega}_{k}-N_{i j k l} \partial \bar{u}_{k} / \partial x_{l}$, where $\Lambda_{i j k}$ and $N_{i j k l}$ are third and fourth order tensors, respectively. The parametrisation of the velocity correlations $Q_{i j}$ thus involves some free coefficients (turbulent viscosity for instance) that need to be set to ensure the closure of the mean-field model. Despite these approximations, mean-field approaches were quite successful in predicting a weak dependence of the surface shear on the rotation rate and a strong correlation with the effective temperature as observed on the main sequence stars (Küker and Rüdiger 2011). In addition, these models have a strong prediction concerning the sense of the differential rotation and predominantly yield solar-like $\Omega(\theta)$ profiles (Kitchatinov and Rüdiger 1999). Anti-solar differential rotation can only be maintained in case of very strong meridional circulation (Kitchatinov and Rüdiger 2004).

Alternatively, stellar differential rotation can be modelled using 3-D hydrodynamical and dynamo models of rotating convection in spherical geometry. In that case, the differential rotation is maintained by the interaction of turbulent convection with rotation. Despite their own limitations (high diffusivities and moderate density contrasts), 3-D models allow to fully take into account the nonlinearities involved in the angular momentum transport. In contrast with mean-field approaches, no parametrisation of Reynolds stresses is required in 3-D simulations. Although a large number of such simulations yield solar-like differential rotation, they have also frequently produced anti-solar differential rotation over a broad range of parameters and model setups (e.g. Gilman 1977, Glatzmaier and Gilman 1982, Aurnou et al. 2007, Steffen and Freytag 2007, Matt et al. 2011, Käpylä et al. 2011, Bessolaz and Brun 2011, Gastine et al. 2013b). The differential rotation direction is suspected to be controlled by the relative contribution of buoyancy and Coriolis force in the global force balance (Gilman 1977, Aurnou et al. 2007). Cases where rotation dominates the force balance yield prograde equatorial azimuthal flows, while a weak rotational influence leads to anti-solar differential rotation. As shown in previous parameter studies, these two regimes can be well separated by a critical convective Rossby number of unity (Gilman 1977), independently of the background density stratification (Gastine and Wicht 2012, Gastine et al. 2013b, hereafter GW12, GWA13).

The present work extends these studies to a broader range of parameters to investigate the zonal flow transition in 3-D models in a systematic way. For the sake of generality, we also incorporate data of different research groups who reported anti-solar differential 
rotation in their models.

\subsection{Hydrodynamical model}

We consider numerical simulations of an anelastic ideal gas in spherical shells rotating at a constant rotation rate $\Omega_{0}$. A fixed entropy contrast $\Delta s$ between the inner and the outer boundary drives the convective motions. Our numerical models are computed using the anelastic spectral code MagIC (Wicht 2002, GW12) that has been validated against hydrodynamical and dynamo benchmarks (Jones et al. 2011). We non-dimensionalise the MHD equations using $\Omega_{0}^{-1}$ as the time unit and the shell thickness $d=r_{o}-r_{i}$ as the reference lengthscale. The anelastic system of equations is then governed by four dimensionless parameters

$$
E=\frac{v}{\Omega_{0} d^{2}}, R a=\frac{g_{o} d^{3} \Delta s}{c_{p} v \kappa}, \operatorname{Pr}=\frac{v}{\kappa}, \operatorname{Pm}=\frac{v}{\lambda},
$$

where $v, \kappa$, and $\lambda$ are the constant kinematic, thermal and magnetic diffusivities and $g_{o}$ is the gravity at the outer boundary. Details of the numerical implementation are extensively discussed by Jones et al. (2011) and GW12. Differential rotation maintained in 3-D models is suspected to be sensitive to the relative contribution of buoyancy and Coriolis force in the force balance. The ratio between these two forces can be roughly assessed by the so-called convective Rossby number, defined by $R o_{c}=\sqrt{R a E^{2} / P r}$.

The converged solution of a numerical simulation is then characterised by several diagnostic parameters. The rms flow velocity is given in units of the Rossby number $R o^{\prime}=$ $u_{\mathrm{rms}}^{\prime} / \Omega_{0} d$, where primed quantities correspond to the non-axisymmetric contribution. The typical flow lengthscale $\ell$ is defined as $\ell=\pi d / \bar{l}_{u}$, where $\bar{l}_{u}$ is the mean spherical harmonic degree obtained from the kinetic energy spectrum (e.g. Christensen and Aubert 2006, Schrinner et al. 2012). A local Rossby number $R o_{\ell}=u_{\mathrm{rms}}^{\prime} / \Omega_{0} \ell$ can then be used to evaluate the relative contribution of inertia and Coriolis force to the global force balance. Differential rotation is quantified by the amplitude of the equatorial surface zonal flow:

$$
\alpha_{e}=\frac{\bar{u}_{\phi}\left(r=r_{o}, \theta=0\right)}{\Omega_{o} r_{o}}=\frac{d \Omega\left(r=r_{o}, \theta=0\right)}{\Omega_{o}},
$$

where overbars denote axisymmetric quantities.

Our previous parameter studies were dedicated to the effects of the density stratification on the differential rotation (GW12, GWA13). They assumed $\operatorname{Pr}=1$ and covered a limited range of Ekman numbers $\left(E=10^{-3}-10^{-4}\right)$. To extend the coverage of the parameter space, we have computed here 150 new cases which span the range of $10^{-5}<E<10^{-2}, 10^{3}<R a<5 \times 10^{8}$ and $\operatorname{Pr} \in[0.1,1,10]$. We consider here nonmagnetic nearly Boussinesq models (i.e. $\left.N_{\rho}=\ln \left(\rho_{\text {bot }} / \rho_{\text {top }}\right)=10^{-2}\right)$ in a thin spherical shell of aspect ratio $\eta=r_{i} / r_{o}=0.6$. To investigate how the magnetic field influences differential rotation, we also consider a few Boussinesq dynamo models with $\eta=0.35$ and $P m=1$.

Furthermore, we include additional data from published studies which encompasses Boussinesq (e.g. Aurnou et al. 2007), anelastic (e.g. Gilman 1977, ASH) and fully compressible 3-D models (Käpylä et al. 2011). To our knowledge, all the data reporting 


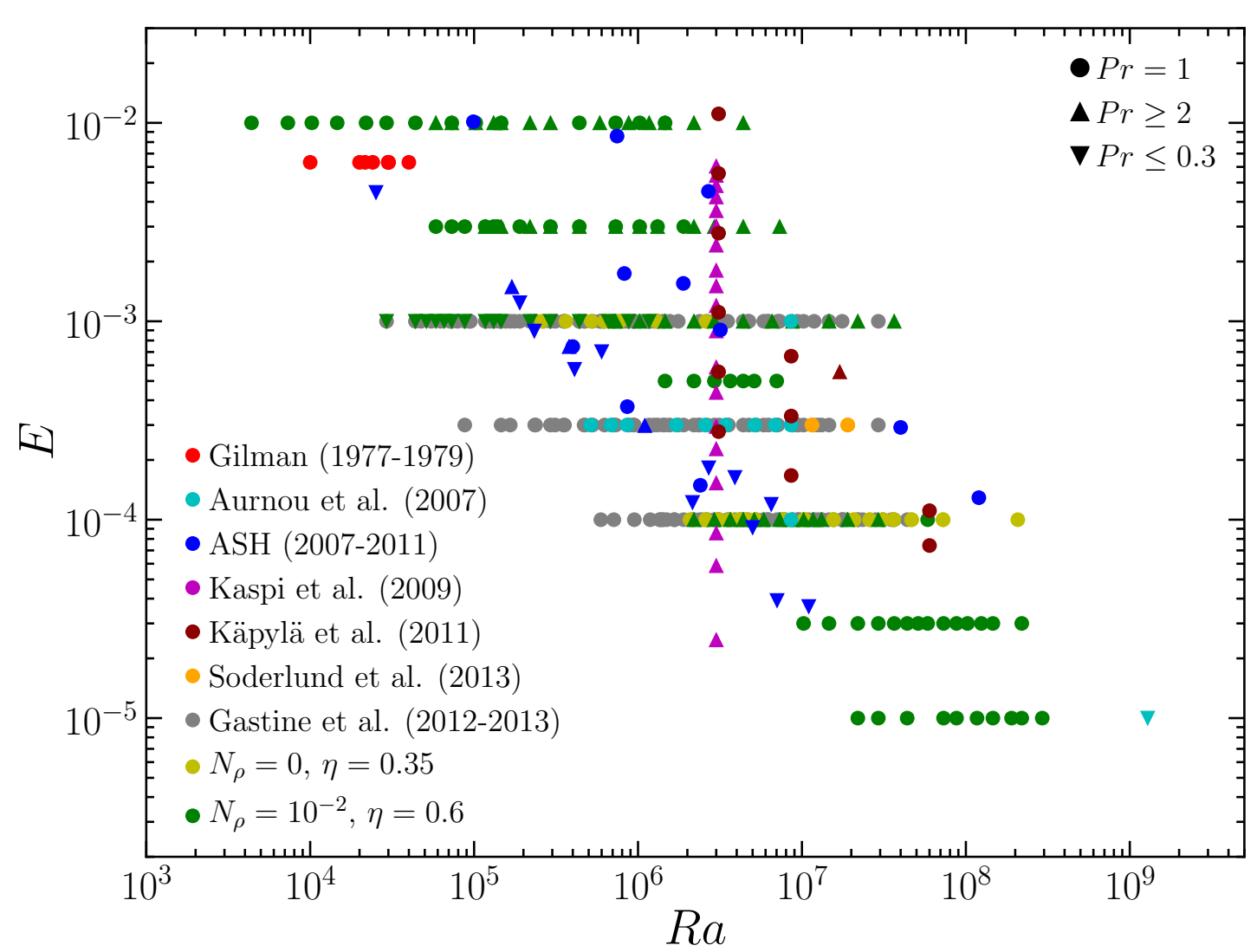

Figure 4.1: Dimensionless control parameters explored by various numerical models computed with different codes. Data have been gathered and adapted from Gilman (1977, 1979); Aurnou et al. (2007), ASH (Ballot et al. 2007, Browning 2008, Brun and Palacios 2009, Brown 2009, Matt et al. 2011, Bessolaz and Brun 2011); Kaspi et al. (2009); Käpylä et al. (2011); Soderlund et al. (2013); GW12 and GWA13.

anti-solar differential rotation have been gathered in Fig. 4.1, provided control and relevant diagnostic parameters (i.e. $\alpha_{e}$ ) were accessible. Note that to ease the comparison between the different setups, the Rayleigh numbers have been rescaled in Fig. 4.1 to use the entropy gradient at mid-depth, i.e. $R a=g_{o} d^{3}|d s / d r|_{m} / c_{p} v \kappa$. This provides a better way of comparing different reference state models (see Kaspi et al. 2009, GWA13).

\subsection{Results}

\subsubsection{Differential rotation regimes}

Figure 4.2 shows the surface differential rotation amplitude $\alpha_{e}$ as a function of $R o_{c}$ for the Fig. 4.1 dataset. When Coriolis forces dominate the force balance (i.e. $R o_{c} \ll 1$, regime I), the equatorial zonal flow is prograde and its amplitude increases with $R o_{c}$. A relatively sharp transition to retrograde zonal winds (or anti-solar differential rotation) then occurs close to $R o_{c} \sim 1$. Although the dataset is scattered, the retrograde equatorial flow is on average stronger than in regime I and reaches values of $\alpha_{e} \sim-0.4$ for $R o_{c} \sim 1$. When 


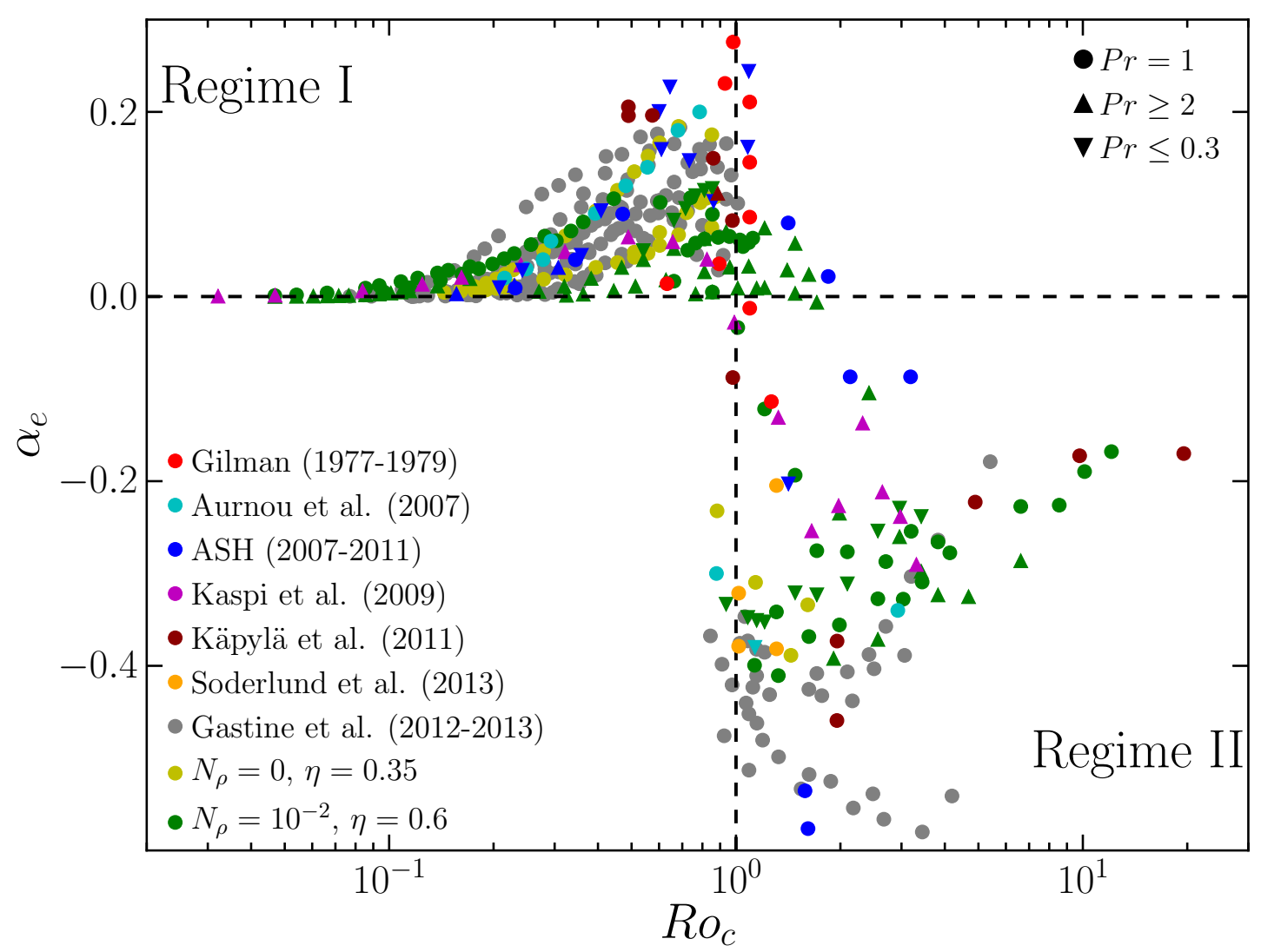

Figure 4.2: Amplitude of the surface zonal flows at the equator in units of $\alpha_{e}=\bar{u}_{\phi} / \Omega_{0} r_{o}$ as a function of $R o_{c}$ for the numerical models of Fig. 4.1.

buoyancy starts to dominate the force balance (i.e. $R o_{c} \gg 1$, regime II), the differential rotation decreases suggesting a possible third regime where turbulent motion gradually suppress the mean flows ( $R o_{c}>10$, see GWA13 and Brummell et al. 1998).

Despite differences in size of the convective layer, values of the control parameters, definition of the Rayleigh number, choice of thermal boundary conditions and so on, the transition between regimes I and II is well captured by $R o_{c}$, with all the data points concentrating in the top-left and bottom-right quadrants.

To illustrate the differences in the differential rotation patterns in the two regimes, Fig. 4.3 shows radial velocity and zonal flows for two selected models. In the rotationdominated regime $\left(R o_{c}=0.09\right.$, upper panels) convective columns aligned with the rotation axis are visible at low latitudes. They are accompanied at higher latitudes by smallscale time-dependent convective cells. Due to the curvature of the spherical shell, the convective columns are slightly tilted in the prograde direction and give rise to Reynolds stresses (a statistical correlation between the convective flow components, see Busse 1983, Christensen 2002). Reynolds stresses maintain a positive flux of angular momentum away from the rotation axis which is responsible for the observed differential rotation. The pair of geostrophic zonal flows with an eastward equator and westward poles is typical in this regime (e.g. Käpylä et al. 2011, GW12). In contrast, when buoyancy becomes a first-order contribution in the force balance ( $R o_{c}=4$, lower panels), the convective 

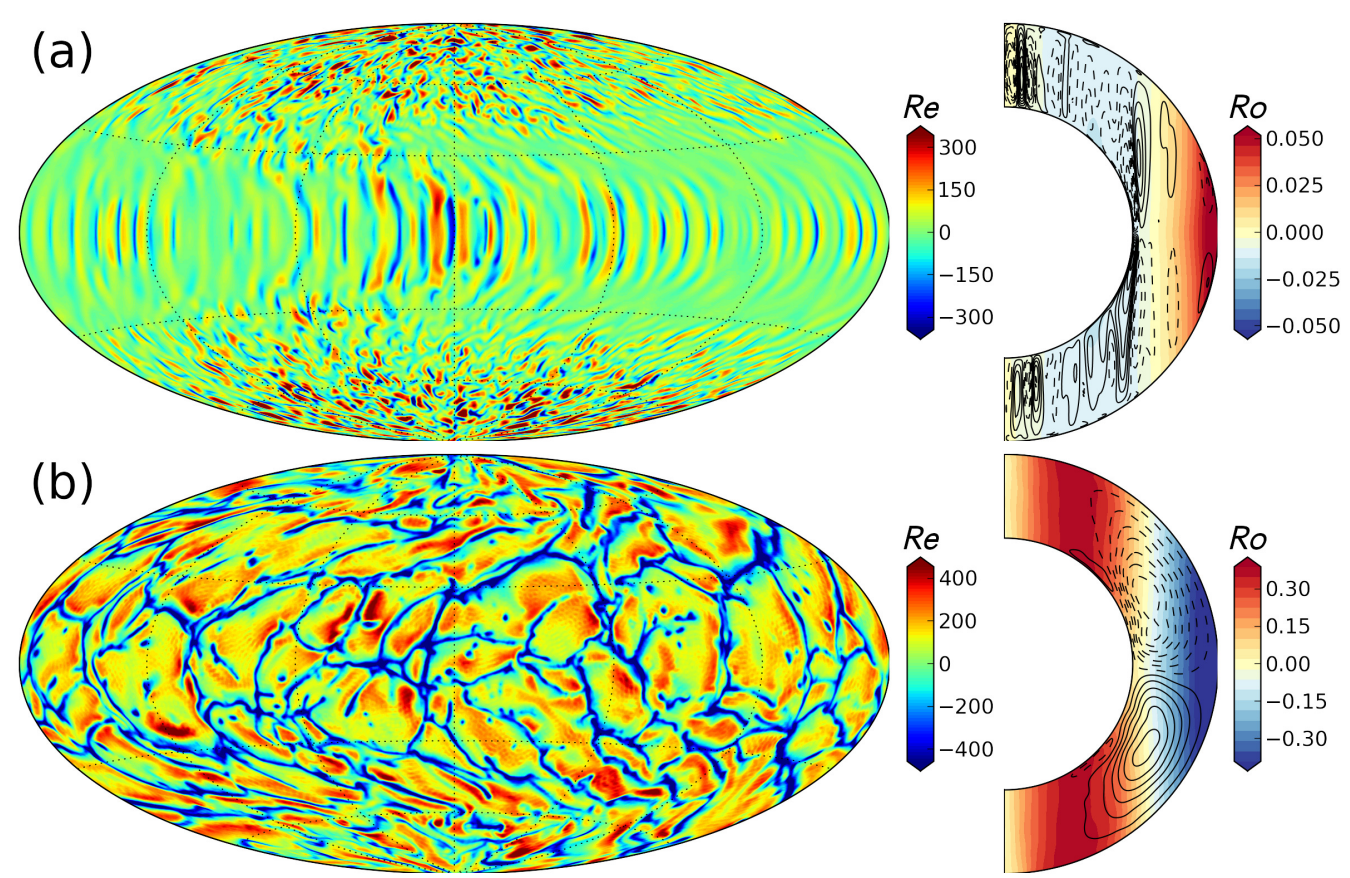

Figure 4.3: Left panels: radial velocity at $r=0.95 r_{o}$. Right panels: time-averaged zonal velocity (colored levels) and meridional circulation (solid and dashed lines). Case (a) corresponds to $N_{\rho}=3, \eta=0.6, E=10^{-4}, R a=9 \times 10^{6}, P r=1$, case (b) to $N_{\rho}=3$, $\eta=0.6, E=10^{-3}, R a=4 \times 10^{6}, P r=1$. Radial velocity is given in Reynolds number units $\left(u_{r} d / v\right)$ while zonal flows are expressed in Rossby number units.

features lose their preferred alignment with the rotation axis and the zonal flow direction reverses. The equatorial jet becomes retrograde and is flanked by two prograde zonal winds inside the tangent cylinder. The anti-solar differential rotation observed here can be attributed to the mixing of angular momentum by the turbulent convective motions (e.g. Gilman and Foukal 1979, Aurnou et al. 2007). As demonstrated by GWA13, the angular momentum per unit mass $\mathcal{M}$ is thus a conserved quantity such that

$$
\mathcal{M}=\bar{u}_{\phi} s+\Omega_{0} s^{2}=\text { const. }=\zeta\left(\eta, N_{\rho}\right) \Omega_{0} r_{o}^{2},
$$

where $s$ is the cylindrical radius and $0<\zeta\left(\eta, N_{\rho}\right)<1$ depends on the background density stratification, the size of the convective zone and the efficiency of the angular momentum mixing. Using $\Omega_{0} r_{o}^{2}$ to non-dimensionalise this equation leads to the following formulation of the differential rotation in regime II:

$$
R o=\frac{\bar{u}_{\phi}}{\Omega_{0} r_{o}}=\zeta\left(\eta, N_{\rho}\right) \frac{r_{o}}{s}-\frac{s}{r_{o}} .
$$

Comparisons between the zonal flow profiles and this theoretical prediction give a good agreement for models with $R o_{c} \gtrsim 1$ (Aurnou et al. 2007, GWA13).

Meridional circulation patterns change when differential rotation changes sign (e.g. Matt et al. 2011, Bessolaz and Brun 2011). In the upper panel of Fig. 4.3, multiple small-scale meridional circulation cells are observed, while the second model shows only one large-scale cell in each hemisphere. This transition results from a change in the 

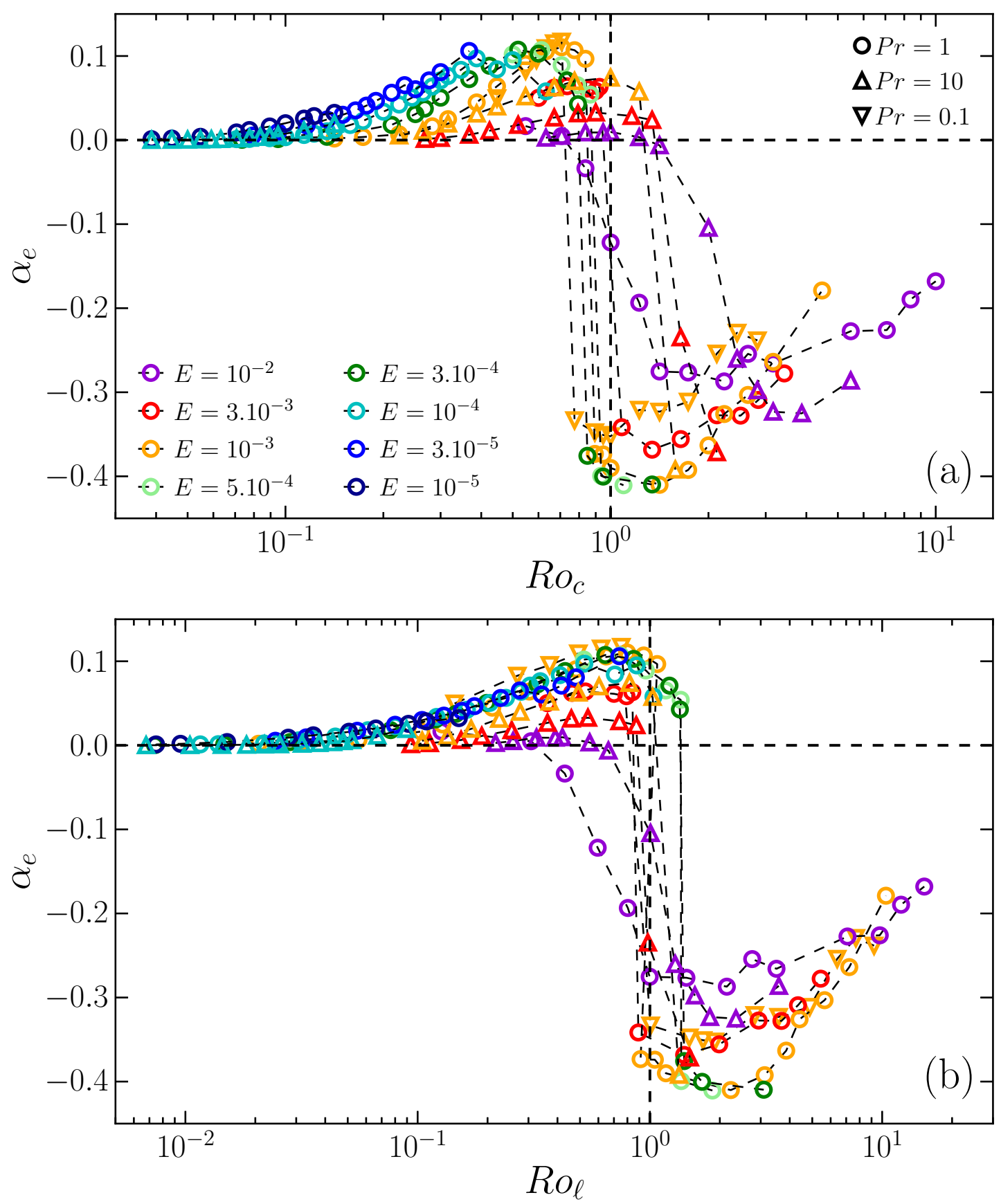

Figure 4.4: (a) $\alpha_{e}$ as a function of $R o_{c}$ and (b) as a function of $R o_{\ell}$. All the numerical models have $N_{\rho}=10^{-2}$ and $\eta=0.6$.

spatial variations of the azimuthal force balance between viscous and Reynolds stresses (a mechanism sometimes known as "gyroscopic pumping", e.g. Miesch and Hindman 2011).

Figure 4.4a shows the same quantities as Fig. 4.2 for a consistent subset of nearly Boussinesq numerical models $\left(N_{\rho}=10^{-2}\right)$ with $\eta=0.6$. This subset is partly composed by the Boussinesq models of GW12 and GWA13 and partly by the additional cases com- 
puted for the present study. Considering the same reference model for the whole subset allows to more accurately scrutinise the zonal flow transition. While the regime change occurs in the range $0.8<R o_{c}<2$, some parameter dependence is still noticeable. For instance, the transition is rather gradual for large Ekman numbers $\left(E=10^{-2}\right.$, magenta symbols) and becomes sharper when the Ekman number is lowered. Moreover, the Prandtl number dependence does not seem to be perfectly captured by $R o_{c}$. In fact, the zonal flow transition in the numerical models with $\operatorname{Pr}=10(\operatorname{Pr}=0.1)$ takes place at higher (lower) values of $R o_{c}$ than the $P r=1$ cases. As our dataset is limited to relatively large Ekman numbers for $\operatorname{Pr} \neq 1$, we might however speculate that such $\operatorname{Pr}$ dependence vanishes at low Ekman numbers. As shown in Fig. 4.4b, the zonal flow transition is better captured when $\alpha_{e}$ is plotted against the local Rossby number $R o_{\ell}$. This reduction of the dispersion is expected as $R o_{c}$ is only a rough proxy of the convective Rossby number, while $R o_{\ell}$ is a measure of the actual local Rossby number of a numerical model. A precise estimate of $R o_{\ell}$ for the whole dataset of models shown in Fig. 4.2 would thus also help to reduce the observed dispersion.

\subsubsection{Zonal flow bistability}

We find several cases of bistability where the two kinds of differential rotation are stable at identical parameters (i.e. $R a, E$ and $\operatorname{Pr}$ ) when $R o_{c} \sim R o_{\ell} \sim 1$. The initial condition then selects which differential rotation profile will be adopted by the converged solution. As shown on Fig. 4.5, starting from a model with a solar-like differential rotation and increasing $R o_{c}$ (or $R o_{\ell}$ ) maintains a solution with the same kind of differential rotation for $0.5<R o_{\ell}<1.3$ before falling on the other branch at higher $R o_{\ell}$. Alternatively, if one initiates this model with $\alpha_{e}<0$ and decreases $R o_{c}$, the solution may remain on that branch. Once again, the $\operatorname{Pr}$ dependence on the bistability region seems to be better captured when one considers $R o_{\ell}$ instead of $R o_{c}$. The hysteresis loop is relatively narrow for $E=10^{-3}$ and becomes wider at $E=3 \times 10^{-4}$. At $E=10^{-4}$, it becomes numerically too demanding to further investigate the extent of the two branches. Hence, an Ekman number dependence cannot be completely ruled out and the extent of the bistability region might increase further when $E$ is lowered.

\subsubsection{Magnetic field influence}

To investigate if the zonal flow transition is affected by the presence of magnetic field, we compute two sets of Boussinesq models with $\eta=0.35$ : one consists of non-magnetic cases, while the other contains their dynamo counterparts. Figure 4.6 shows that in both cases the transition between regimes I and II occurs around $R o_{\ell} \sim 1$. Due to the influence of the magnetic field on the convective flow velocity and lengthscale, the exact value of $R o_{\ell}$ at the transition is slightly lower in the dynamo models. In the rotation-dominated regime, the magnetic cases have significantly weaker zonal flows than the non-magnetic ones. In contrast, hydrodynamical and dynamo models yield similar zonal flow amplitude in regime II, confirming the previous findings by Soderlund et al. (2013). These differences can be attributed to the relative efficiency of the magnetic braking. The quenching of the differential rotation by Lorentz forces is indeed more pronounced when the magnetic field has a significant large-scale contribution $\left(R o_{\ell}<1\right.$, Yadav et al. 2013). 

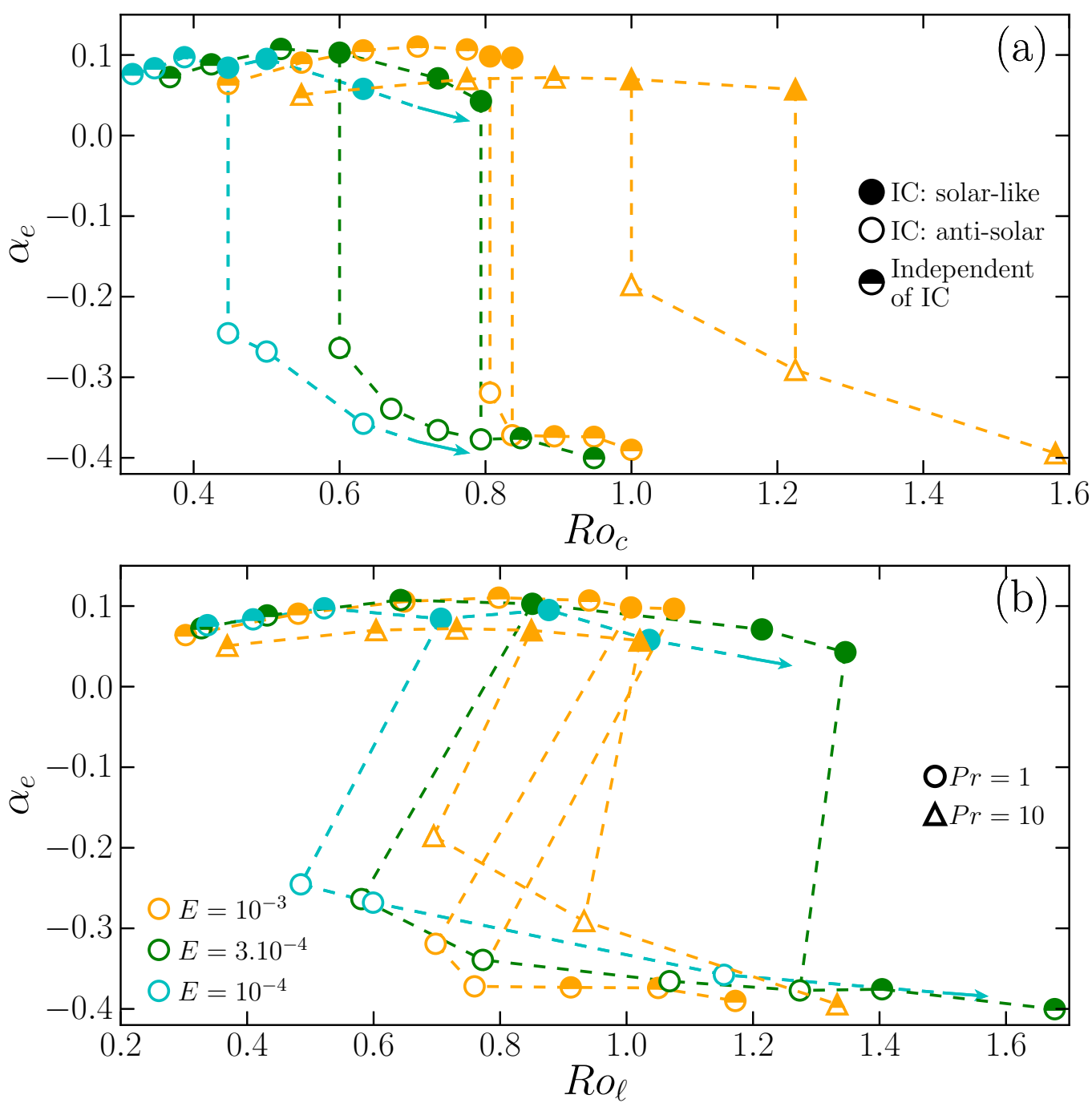

Figure 4.5: (a) $\alpha_{e}$ as a function of $R o_{c}$ and (b) as a function of $R o_{\ell}$. Selection of numerical models with $N_{\rho}=10^{-2}$ and $\eta=0.6$ to illustrate the bistability of the zonal flow. The dependence on the initial conditions (IC) is shown by different symbol fillstyles. The blue arrows indicate possible continuation of the hysteresis loop for the $E=10^{-4}$ cases. 


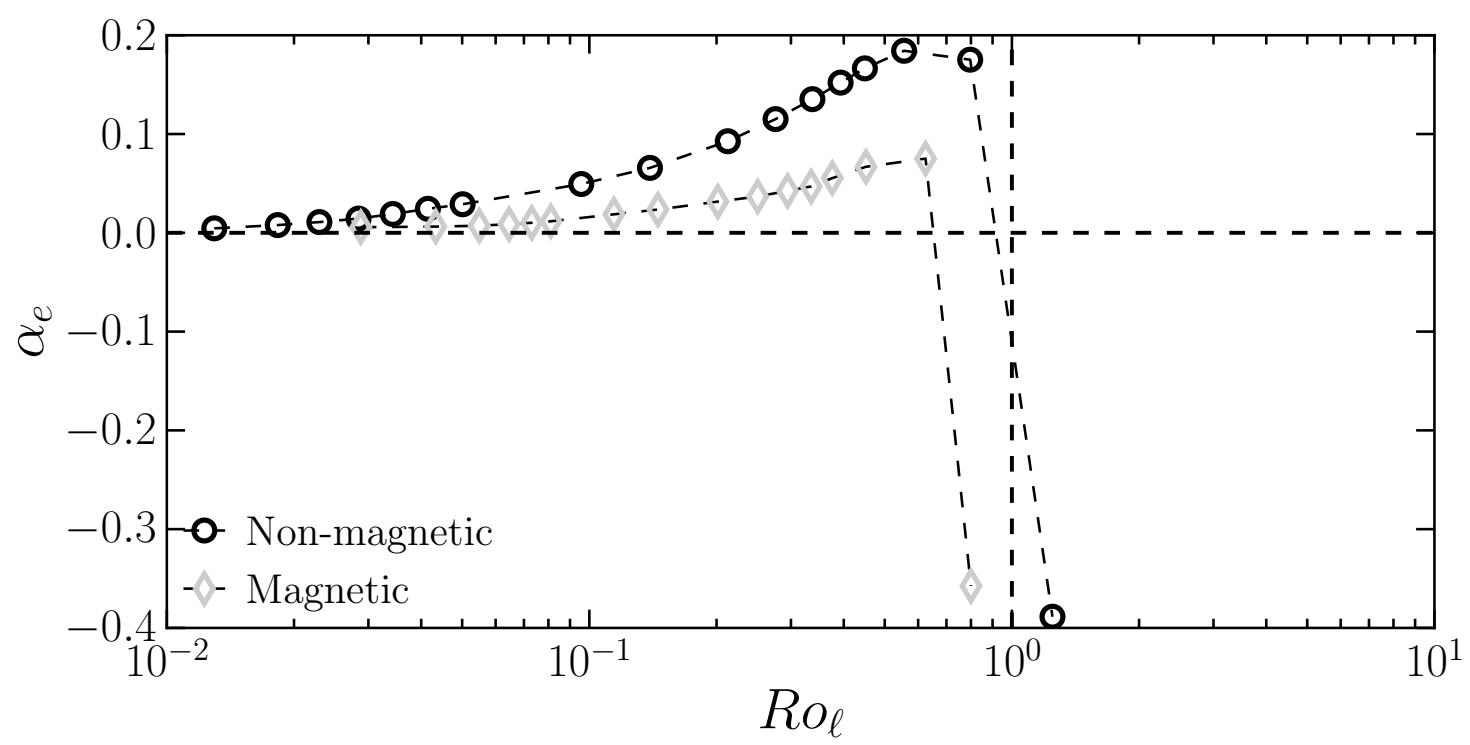

Figure 4.6: $\alpha_{e}$ as a function of $R o_{\ell}$ for non-magnetic and magnetic $(P m=1)$ models with $N_{\rho}=0, \eta=0.35, E=10^{-4}$ and $\operatorname{Pr}=1$.

\subsection{Discussion}

We investigate the transition between solar-like and anti-solar differential rotation in rotating spherical shells. We extend previous studies (Gastine and Wicht 2012, Gastine et al. $2013 \mathrm{~b}$ ) with a new set of models which covers a broader range of control parameters. We also include models published by various groups in our analysis.

From this set of simulations we confirm previous findings that the direction of differential rotation is determined by the value of the convective Rossby number defined as $R o_{c}=\sqrt{R a E^{2} / P r}$. In the rotation-dominated regime (regime I, $R o_{c}<1$ ), the differential rotation is solar-like, i.e. the equator rotates faster than the poles. When buoyancy dominates the force balance $\left(R o_{c}>1\right)$, the turbulent convective motions homogenise the angular momentum, which leads to anti-solar differential rotation profiles. The regime transition takes place at $R o_{c} \sim 1$, independently of the details of the model (density stratification, thickness of the convective layer and so on). We show that the local Rossby number $R o_{\ell}$ - a good proxy of the relative contribution of Coriolis force and inertia in the force balance (Christensen and Aubert 2006) - helps to better separate the two regimes. Close to the transition $\left(0.5<R o_{\ell}<1.5\right)$, the two kinds of differential rotation are two possible stable states at the same parameter values, forming a bistable region. The presence of a magnetic field reduces the amplitude of differential rotation in regime I without affecting the regime change at $R o_{\ell} \sim 1$.

It should be however noted that global numerical models always operate in a parameter regime far from the stellar values due to their large diffusivities (i.e. small Rayleigh and large Ekman numbers). Hence, the existence of additional dynamical regimes cannot be ruled out at realistic parameters. Nonetheless, anti-solar differential rotation is systematically found in weakly-rotating 3-D simulations in contrast with the mean-field results. A closer comparison between mean-field predictions and 3-D simulations is therefore desirable to better establish the limits of validity of such mean-field approaches (e.g. Käpylä 
et al. 2011).

Our results provide theoretical support for the existence of slowly rotating cool stars exhibiting anti-solar differential rotation. A further validation of our prediction requires to estimate $R o_{\ell}$ in stellar convective zones. We adopt $R o_{\text {emp }}$, the ratio of the rotation period $P_{\text {rot }}$ and the turnover time of convection $\tau_{\text {conv }}$, as our best available proxy for $R o_{\ell}$ (e.g. Gastine et al. 2013a). With $P_{\text {rot }}=25 \mathrm{~d}$ and $\tau_{\text {conv }}=12-50 \mathrm{~d}$ (Reiners 2012), the solar Rossby number lies in the range $0.5<R o_{\text {emp }}<2$. This suggests that the Sun might be at the limit of the rotation-dominated regime and that stars with Rossby number just above the solar value could exhibit strong anti-solar differential rotation. Claims of anti-solar differential rotation are so far restricted to K giants. Most of these stars are in binary systems where tidal effects likely have an impact on the surface shear (e.g. Kovári et al. 2007). The K giant HD 31993 is the only single giant for which a significant antisolar differential rotation is reported ( $\alpha=-0.125$, Strassmeier et al. 2003). For this star $P_{\text {rot }}=25.3 \mathrm{~d}$ and $\tau_{\text {conv }} \simeq 25 \mathrm{~d}$ (Gunn et al. 1998) yield $R o_{\text {emp }} \simeq 1$, a value close to the threshold but compatible with $\alpha<0$.

Measuring differential rotation for stars clearly in the $R o>1$ regime remains challenging. Doppler imaging or line profile analysis are sensitive to the sign of differential rotation but suffer from some limitations. Doppler imaging indeed relies on the presence of large spots at the stellar photosphere which is not expected for $R o_{\text {emp }}>1$. Line profile analysis requires a minimum rotational velocity $v \sin i_{\min } \sim 10-20 \mathrm{~km} . \mathrm{s}^{-1}$. This is incompatible with $R o_{\text {emp }}>1$ for cool main sequence stars. Although Ammler-von Eiff and Reiners (2012) observed line profile shapes attributable to $\alpha \lesssim 0$ for dwarf stars with $R o_{\text {emp }}<1$, they attributed these signatures to the presence of cool polar spots. With their larger radii, weakly active evolved giant stars might be more suitable targets. Space missions CoRoT and Kepler collect high-precision photometric data for a vast sample of stars. Although this technique cannot directly determine the sign of $\alpha$, a regime change in the differential rotation might still be captured. Our numerical models indeed suggest a relatively sharp rise in $|\alpha|$ at the transition between solar and anti-solar differential rotation. Latest results based on moderate to fast rotators $\left(P_{\text {rot }}<45 \mathrm{~d}\right)$ by Reinhold et al. (2013) suggest a possible increase of $|\alpha|$ with the Rossby number stressing the need for further analysis of slowly-rotating Kepler stars.

\section{Acknowledgements}

We thank P. Käpylä for providing us the parameters of his numerical models and A. S. Brun for fruitful discussion. Computations have been carried out on the GWDG computer facilities in Göttingen and on HRLN in Hannover. We acknowledge funding from the Deutsche Forschungsgemeinschaft (DFG) through Project SFB 963/A17 and through the Special Priority Program 1488. 


\section{Bibliography}

Ammler-von Eiff, M. and Reiners, A. (2012). New measurements of rotation and differential rotation in A-F stars: are there two populations of differentially rotating stars? $A \mathcal{E} A, \mathbf{5 4 2}, \mathrm{A} 116$.

Aurnou, J., Heimpel, M., and Wicht, J. (2007). The effects of vigorous mixing in a convective model of zonal flow on the ice giants. Icarus, 190, 110-126.

Ballot, J., Brun, A. S., and Turck-Chièze, S. (2007). Simulations of turbulent convection in rotating young solarlike stars: differential rotation and meridional circulation. ApJ, 669, 1190-1208.

Barnes, J. R., Collier Cameron, A., Donati, J.-F., James, D. J., Marsden, S. C., and Petit, P. (2005). The dependence of differential rotation on temperature and rotation. MNRAS, 357, L1-L5.

Bessolaz, N. and Brun, A. S. (2011). Hunting for Giant Cells in Deep Stellar Convective Zones Using Wavelet Analysis. ApJ, 728, 115.

Brown, B. P. (2009). Convection and Dynamo Action in Rapidly Rotating Suns. Ph.D. thesis, University of Colorado, USA.

Browning, M. K. (2008). Simulations of Dynamo Action in Fully Convective Stars. ApJ, 676, 1262-1280.

Brummell, N. H., Hurlburt, N. E., and Toomre, J. (1998). Turbulent Compressible Convection with Rotation. II. Mean Flows and Differential Rotation. ApJ, 493, 955.

Brun, A. S. and Palacios, A. (2009). Numerical Simulations of a Rotating Red Giant Star. I. Three-dimensional Models of Turbulent Convection and Associated Mean Flows. ApJ, 702, 1078-1097.

Busse, F. H. (1983). A model of mean zonal flows in the major planets. GAFD, 23, 153-174.

Christensen, U. R. (2002). Zonal flow driven by strongly supercritical convection in rotating spherical shells. Journal of Fluid Mechanics, 470, 115-133.

Christensen, U. R. and Aubert, J. (2006). Scaling properties of convection-driven dynamos in rotating spherical shells and application to planetary magnetic fields. Geophys. J. Int., 166, 97-114. 
Collier Cameron, A., Donati, J.-F., and Semel, M. (2002). Stellar differential rotation from direct star-spot tracking. MNRAS, 330, 699-706.

Gastine, T. and Wicht, J. (2012). Effects of compressibility on driving zonal flow in gas giants. Icarus, 219, 428-442.

Gastine, T., Morin, J., Duarte, L., Reiners, A., Christensen, U. R., and Wicht, J. (2013a). What controls the magnetic geometry of M dwarfs? AEA A, 549, L5.

Gastine, T., Wicht, J., and Aurnou, J. M. (2013b). Zonal flow regimes in rotating anelastic spherical shells: An application to giant planets. Icarus, 225, 156-172.

Gilman, P. A. (1977). Nonlinear Dynamics of Boussinesq Convection in a Deep Rotating Spherical Shell. I. GAFD, 8, 93-135.

Gilman, P. A. (1979). Model calculations concerning rotation at high solar latitudes and the depth of the solar convection zone. ApJ, 231, 284-292.

Gilman, P. A. and Foukal, P. V. (1979). Angular velocity gradients in the solar convection zone. ApJ, 229, 1179-1185.

Glatzmaier, G. A. and Gilman, P. A. (1982). Compressible convection in a rotating spherical shell. V - Induced differential rotation and meridional circulation. ApJ, 256, 316330 .

Gunn, A. G., Mitrou, C. K., and Doyle, J. G. (1998). On the rotation-activity correlation for active binary stars. MNRAS, 296, 150-164.

Jones, C. A., Boronski, P., Brun, A. S., Glatzmaier, G. A., Gastine, T., Miesch, M. S., and Wicht, J. (2011). Anelastic convection-driven dynamo benchmarks. Icarus, 216, $120-135$.

Käpylä, P. J., Mantere, M. J., Guerrero, G., Brandenburg, A., and Chatterjee, P. (2011). Reynolds stress and heat flux in spherical shell convection. AEFA, 531, A162.

Kaspi, Y., Flierl, G. R., and Showman, A. P. (2009). The deep wind structure of the giant planets: Results from an anelastic general circulation model. Icarus, 202, 525-542.

Kitchatinov, L. L. and Rüdiger, G. (1999). Differential rotation models for late-type dwarfs and giants. $A \mathcal{E} A$, 344, 911-917.

Kitchatinov, L. L. and Rüdiger, G. (2004). Anti-solar differential rotation. AN, 325, 496-500.

Kovári, Z., Bartus, J., Strassmeier, K. G., Vida, K., Švanda, M., and Oláh, K. (2007). Anti-solar differential rotation on the active K-giant $\sigma$ Geminorum. AEA, 474, 165168.

Küker, M. and Rüdiger, G. (2011). Differential rotation and meridional flow on the lower zero-age main sequence: Reynolds stress versus baroclinic flow. AN, 332, 933. 
Matt, S. P., Do Cao, O., Brown, B. P., and Brun, A. S. (2011). Convection and differential rotation properties of $\mathrm{G}$ and $\mathrm{K}$ stars computed with the ASH code. AN, 332, 897.

Miesch, M. S. and Hindman, B. W. (2011). Gyroscopic Pumping in the Solar Near-surface Shear Layer. ApJ, 743, 79.

Reiners, A. (2012). Observations of Cool-Star Magnetic Fields. Living Reviews in Solar Physics, 9, 1.

Reiners, A. and Schmitt, J. H. M. M. (2002). On the feasibility of the detection of differential rotation in stellar absorption profiles. AEFA, 384, 155-162.

Reinhold, T., Reiners, A., and Basri, G. (2013). Rotation and differential rotation of active Kepler stars. ArXiv e-prints.

Ruediger, G. (1989). Differential rotation and stellar convection. Sun and the solar stars.

Schrinner, M., Petitdemange, L., and Dormy, E. (2012). Dipole Collapse and Dynamo Waves in Global Direct Numerical Simulations. ApJ, 752, 121.

Soderlund, K. M., Heimpel, M. H., King, E. M., and Aurnou, J. M. (2013). Turbulent models of ice giant internal dynamics: Dynamos, heat transfer, and zonal flows. Icarus, 224, 97-113.

Steffen, M. and Freytag, B. (2007). Rotating 'star-in-a-box' experiments. AN, 328, 1054 1058.

Strassmeier, K. G., Kratzwald, L., and Weber, M. (2003). Doppler imaging of stellar surface structure. XX. The rapidly-rotating single K2-giant HD $31993=\mathrm{V} 1192$ Orionis. $A \mathcal{E} A, \mathbf{4 0 8}, 1103-1113$.

Thompson, M. J., Christensen-Dalsgaard, J., Miesch, M. S., and Toomre, J. (2003). The Internal Rotation of the Sun. ARAE $A, 41,599-643$.

Weber, M., Strassmeier, K. G., and Washuettl, A. (2005). Indications for anti-solar differential rotation of giant stars. AN, 326, 287-291.

Wicht, J. (2002). Inner-core conductivity in numerical dynamo simulations. Physics of the Earth and Planetary Interiors, 132, 281-302.

Yadav, R. K., Gastine, T., and Christensen, U. R. (2013). Scaling laws in spherical shell dynamos with free-slip boundaries. Icarus, 225, 185-193. 


\title{
5 Formation of starspots in self-consistent global dynamo models: Polar spots on cool stars
}

\begin{abstract}
:
Context - Observations of cool stars reveal dark spot-like features on their surfaces. These starspots can be more extended than sunspots and cover a large area of the stellar surface. While sunspots appear only at low latitudes, starspots are also found in polar regions, in particular on rapidly rotating stars. Conventional flux-tube models have been invoked to explain starspot properties. However, these models use several simplifications, and so far, neither sunspots nor starspots have been generated in a self-consistent simulation of stellar magnetic convection.
\end{abstract}

Aims - We aim to clarify the conditions necessary for the spontaneous formation of dark spots in numerical models of convection-driven stellar dynamos.

Methods - We simulated convection and magnetic field generation in rapidly rotating spherical shells assuming anelastic approximation. The high-resolution simulations were performed using a fully spectral magnetohydrodynamic code.

Results - We demonstrate for the first time that a self-consistent distributed dynamo can spontaneously generate high-latitude dark spots. Dark spots are generated when a largescale magnetic field, generated in the bulk of the convection zone, interacts with and locally quenches flow near the surface. Sufficiently strong density stratification and rapid rotation are prerequisites for the formation of sizeable dark spots in the model.

Conclusions - Our models present an alternative scenario for starspot formation by distributed dynamo action. Our results also lend strong support to the idea that dynamos in the interiors of rapidly rotating stars might be fundamentally different from the solar one ${ }^{1,2}$.

\footnotetext{
${ }^{1}$ The content of this chapter has been reproduced from: R. K. Yadav, T. Gastine, U. R. Christensen, and A. Reiners, "Formation of starspots in self-consistent global dynamo models: Polar spots on cool stars", A\&A 573, A68, 2015. DOI: 10.1051/0004-6361/201424589

${ }^{2}$ Contribution: I ran most of the simulations used in the study and carried out the analysis. The initial part of model A-Pr10-N5 was first ran by Thomas Gastine (although for different purpose). Thomas Gastine also implemented a feature in the simulation code to generate data for Figure 5.6. Uli Christensen converted the non-dimensional input and output parameters to physical units for a discussion in Sec. 5.4 of this chapter. I wrote the first draft of the manuscript; despite changes in later revisions the original structure of the manuscript was preserved.
} 


\subsection{Introduction}

Dark spots are among the most remarkable features on the surface of the Sun. Sunspots are believed to be caused by the interaction of the solar magnetic field with near-surface turbulent convection: in regions of a strong magnetic field, convection is highly quenched, which leads to inefficient transport of heat, which in turn forms local cool and dark spots (see Stein 2012). The size and distribution of regions with a strong magnetic field are ultimately governed by the underlying dynamo mechanism. In the current popular picture of the solar dynamo the interface region of strong radial shear between the radiative core and the convective envelope, the tachocline, is thought to be instrumental (Charbonneau 2005). The tachocline creates a strong toroidal magnetic field by shearing the poloidal field lines. Thin flux-tubes with strong magnetic fields detach from the tachocline and rise to the solar surface where they provide the magnetic field necessary for forming sunspots.

In the past few decades, observational techniques such as photometric light-curve modelling and Doppler imaging (Vogt et al. 1987) have been applied to infer dark starspots on other cool stars. Light-curve modelling is inherently ambiguous ${ }^{3}$, and gathering goodquality data for Doppler imaging is rather tedious. The evolution of starspots (which appear and disappear during the observations) makes it even more complicated. Nonetheless, there are a few reliable features for which support has been found over the years (see Berdyugina (2005), Strassmeier (2009), and reference therein). A particularly intriguing feature is that starspots occur near the rotational poles of many rapidly rotating stars (Strassmeier 2002). This behaviour is in stark contrast with sunspots, which appear exclusively at low latitudes. Starspots in these stars also appear to be rather large, sometimes covering as much as a few percent of the stellar surface, while the collective area of sunspots even during the solar maximum covers only a small fraction of a percent of the solar photosphere.

By extending the flux-tube models to rapidly rotating cool stars, it has been suggested that rising flux-tubes appear at high latitudes either as a result of the influence of strong Coriolis forces (Schüssler and Solanki 1992, Işik et al. 2011), or that they appear at low latitudes and are then advected polewards by near-surface flows (Schrijver and Title 2001, Holzwarth et al. 2006, Işik et al. 2007, 2011). Mechanism based on magnetic Rossby waves in the tachocline of rapidly rotating stars have also been proposed for polar spot formation (Zaqarashvili et al. 2011).

In contemporary flux-tube models, the formation of dark spots is not considered. These models only provide information about the background magnetic field that supposedly leads to dark spot formation. On the other hand, direct numerical simulations of distributed dynamos and dark spot formation have been rather disconnected. Global dynamo models (e.g. Miesch 2005, Steffen and Freytag 2007, Ghizaru et al. 2010, Käpylä et al. 2012, Gastine et al. 2012, Nelson et al. 2013, Hotta et al. 2014, Fan and Fang 2014) simulate the generation of large-scale magnetic fields, while local simulations (e.g. Vögler et al. 2005, Stein and Nordlund 2006, Rempel et al. 2009, Kitiashvili et al. 2010, Cheung et al. 2010, Stein et al. 2011) study the formation of dark spots in the presence of a prescribed magnetic field. Mitra et al. (2014) recently simulated a self-consistent dynamo using an artificially forced flow in a box-geometry; the resulting magnetic field

\footnotetext{
${ }^{3}$ However, see Davenport et al. (2014), who used light curves featuring starspot-occulting planetary transits to separate some of the degeneracies of light-curve modelling.
} 
was able to quench flow in a localized region in the box, mimicking dark spot formation. Although this is certainly a step in the right direction, the setup is simplistic and does not incorporate rotation or convection.

The idea that stellar dynamos necessarily rely on strong shear flow in a tachocline region is certainly worth revisiting, given that flux-tube models are contested even in the solar context (Brandenburg 2005). Furthermore, recent high-resolution numerical simulations of distributed dynamos in spherical shells are also providing a rather different perspective on the solar dynamo (Ghizaru et al. 2010, Käpylä et al. 2012, Nelson et al. 2013). It has been suggested that dynamos in stars in which rotation plays a dominant role might be fundamentally different from the solar case (Christensen et al. 2009, Donati and Landstreet 2009, Reiners 2012). Similar to the dynamos thought to be working in planetary interiors, a distributed dynamo in stars that pervades the entire convection zone can potentially avoid many of the shortcomings of the flux-tube models. For instance, it is hard to imagine how a tachocline region around a geometrically small radiative core in stars (e.g. young pre-main sequence stars or low-mass stars) could govern the dynamo. Flux-tube models that were extended to such stars produced magnetic fields only near the rotational poles (Holzwarth 2004). For fully convective stars the flux-tube picture clearly does not apply. A distributed dynamo, on the other hand, does not need a tachocline and hence can easily operate in stars with small or no radiative cores.

To investigate the generation of a global magnetic field in spherical-shell convection and the simultaneous formation of cool surface spots self-consistently, we further advance a dynamo model that has been applied to gas planets and cool stars (Gastine et al. 2012, 2013a). We do not attempt to model a specific type of star or to match stellar structure and properties as faithfully as possible. Hence this study is in essence exploratory, to find out which basic ingredients are necessary to spontaneously generate dark spots in global numerical simulations without a tachocline. Our main focus is to produce large starspots at high latitudes in the framework of distributed dynamo models.

It is rather tempting to envisage a scenario for polar spots where largely axisymmetric and dipolar magnetic fields, similar in geometry to the fields of Earth or Jupiter, are the backbone. In such dynamos the magnetic field strength naturally peaks at high latitudes. We adopt this as our working hypothesis and pursue it to generate polar spots.

\subsection{Model setup}

\subsubsection{Anelastic equations}

We used the anelastic magnetohydrodynamic (MHD) equations (Braginsky and Roberts 1995, Lantz and Fan 1999) to simulate the subsonic flows below the photosphere of a star. An electrically conducting fluid convects under the influence of a fixed entropy contrast across a spherical shell with an inner radius $r_{i}$ and outer radius $r_{o}$. The shell rotates with a constant angular velocity $\Omega$ about the $z$-axis.

The model equations are non-dimensionalised, using the shell thickness $d=r_{o}-r_{i}$ as the reference length scale and the viscous diffusion time $d^{2} / v$ ( $v$ is viscosity) as the time scale. The density at the outer boundary $\rho_{o}$ is used as the density unit and $\sqrt{\Omega \lambda \mu \rho_{o}}$ is the magnetic field scale. The pressure is scaled by $\rho_{o} \nu \Omega$. The magnetic diffusivity $\lambda$ and the 
magnetic permeability $\mu$ are assumed to be constant. The imposed entropy contrast $\Delta s$ between the inner and the outer boundary defines the entropy scale.

In the anelastic approximation the thermodynamic variables, density, and pressure, are decomposed into a sum of reference state values and small perturbations as $x(r, \theta, \phi, t)=$ $\tilde{x}(r)+x^{\prime}(r, \theta, \phi, t)$. The reference state $\tilde{x}(r)$ corresponds to an adiabatic ideal gas. The reference state density $\tilde{\rho}$ and temperature $\tilde{T}$ are then related by $\tilde{\rho}=\tilde{T}^{m}$, where $m$ is the polytropic index. Assuming a linear variation of gravity, the reference state temperature is given by

$$
\tilde{T}=1-\frac{D i}{2(1-\eta)}\left(\frac{r^{2}}{r_{o}^{2}}-1\right)
$$

where

$$
D i=\frac{g_{o} d}{c_{p} T_{o}}=2 \frac{e^{\frac{N_{\rho}}{m}}-1}{1+\eta}
$$

is the dissipation number, $g_{o}$ is the gravity at the outer boundary, $c_{p}$ is the specific heat at constant pressure, $N_{\rho}=\ln \left(\tilde{\rho}\left(r_{i}\right) / \tilde{\rho}\left(r_{o}\right)\right)$ is the number of density scale heights across the shell, and $\eta=r_{i} / r_{o}$ is the aspect ratio of the spherical shell.

The non-dimensional evolution equation for velocity $\mathbf{u}$ is

$$
\begin{gathered}
E\left(\frac{\partial \mathbf{u}}{\partial t}+\mathbf{u} \cdot \nabla \mathbf{u}\right)=-\nabla \frac{p^{\prime}}{\tilde{\rho}}-2 \hat{z} \times \mathbf{u}+\frac{\operatorname{Ra} E}{\operatorname{Pr}} \frac{r}{r_{o}} s \hat{r}+ \\
\frac{1}{P m \tilde{\rho}}(\nabla \times \mathbf{B}) \times \mathbf{B}+\frac{E}{\tilde{\rho}} \nabla \cdot \mathrm{S},
\end{gathered}
$$

where $p$ is the pressure, $\mathbf{B}$ is the magnetic field, $s$ is the specific entropy, and $\hat{r}$ is the radial unit vector.

$$
\mathrm{S}_{i j}=\tilde{\rho}\left(\frac{\partial u_{i}}{\partial x_{j}}+\frac{\partial u_{j}}{\partial x_{i}}-\frac{2}{3} \delta_{i j} \nabla \cdot \mathbf{u}\right)
$$

is the traceless rate-of-strain tensor. The entropy is governed by

$$
\tilde{\rho} \tilde{T}\left(\frac{\partial s}{\partial t}+\mathbf{u} \cdot \nabla s\right)=\frac{1}{\operatorname{Pr}} \nabla \cdot(\tilde{\rho} \tilde{T} \nabla s)+\frac{\operatorname{Pr} D i}{\operatorname{Ra}}\left[\mathrm{S}^{2}+\frac{1}{E \operatorname{Pm}^{2}}(\nabla \times \mathbf{B})^{2}\right] .
$$

The magnetic field $\mathbf{B}$ evolves according to the induction equation

$$
\frac{\partial \mathbf{B}}{\partial t}=\nabla \times(\mathbf{u} \times \mathbf{B})+\frac{1}{P m} \nabla^{2} \mathbf{B} .
$$

The set of equations is completed by

$$
\nabla \cdot \mathbf{B}=0 \text { and } \nabla \cdot(\tilde{\rho} \mathbf{u})=0 .
$$

The non-dimensional control parameters appearing in above equations are

$$
\begin{gathered}
\text { the magnetic Prandtl number } P m=\frac{v}{\lambda}, \\
\text { the Prandtl number } \operatorname{Pr}=\frac{v}{\kappa}, \\
\text { the Rayleigh number } \operatorname{Ra}=\frac{g_{o} d^{3} \Delta s}{c_{p} v \kappa}, \\
\text { the Ekman number } E=\frac{v}{\Omega d^{2}},
\end{gathered}
$$


where $\kappa$ is thermal diffusivity. The transport coefficients $v, \lambda, \kappa$ are assumed constant. To better model magnetic-field-driven structures in outer layers, we used a constant $\lambda$. In contrast, earlier studies of stellar dynamos usually assumed a radially increasing $\lambda$, making coupling of flow and magnetic field weaker in outer layers (e.g. Browning 2008, Fan and Fang 2014). The aspect ratio $\eta=r_{i} / r_{o}$ was set to 0.35 for all the simulations reported below.

Following earlier studies (e.g. Fan and Fang 2014), we also report the various energy fluxes that appear in systems governed by the anelastic equations: the entropy diffusion flux $F_{\text {diff }}$, the enthalpy flux $F_{c o n v}$, the kinetic energy flux $F_{K E}$, the viscous diffusion flux $F_{\text {visc }}$, the Poynting flux $F_{\text {poyn }}$, and the resistive flux $F_{\text {res }}$. In our non-dimensional units the various fluxes are defined as follows:

$$
\begin{gathered}
F_{\text {diff }}=-\frac{1}{\operatorname{Pr}} \tilde{\rho} \tilde{T}[\nabla s]_{r}, \\
F_{\text {conv }}=\tilde{\rho} \tilde{T} s u_{r}+\frac{\operatorname{Pr} D i}{E R a} P^{\prime} u_{r}, \\
F_{K E}=\frac{\operatorname{Pr} D i}{\operatorname{Ra}} u_{r}\left(\frac{\tilde{\rho} u^{2}}{2}\right), \\
F_{v i s c}=-\frac{\operatorname{Pr} D i}{\operatorname{Ra}}[\mathbf{u} \cdot \mathrm{S}]_{r}, \\
F_{\text {poyn }}=-\frac{\operatorname{Pr} D i}{\operatorname{Ra} E \operatorname{Pm}}[(\mathbf{u} \times \mathbf{B}) \times \mathbf{B}]_{r}, \\
F_{r e s}=\frac{\operatorname{Pr} D i}{\operatorname{Ra} E \operatorname{Pm} m^{2}}[(\nabla \times \mathbf{B}) \times \mathbf{B}]_{r},
\end{gathered}
$$

where $[\ldots]_{r}$ represents the radial component.

\subsubsection{Boundary conditions}

Both inner and outer boundaries are impenetrable, stress-free, and electrically insulating. Constant entropy is assumed on both boundaries. Unlike previous numerical studies of stellar convection (Browning et al. 2006, Ghizaru et al. 2010, Käpylä et al. 2010, Masada et al. 2013), we did not model a convectively stable layer below the convection zone. The region below the inner boundary is treated as static.

It is worth noting that our choice of constant entropy on the outer boundary allows the possibility of forming dark spots where heat flux is lower than in neighbouring regions. This was not possible in earlier studies of stellar convection zones where constant heat flux boundary conditions were employed.

\subsubsection{Initial conditions}

Global dynamo simulations (Simitev and Busse 2009, Sasaki et al. 2011, Schrinner et al. 2012, Gastine et al. 2013a, 2012, Yadav et al. 2013b) in spherical shells with free-slip boundaries show bistability. In the bistable regime, dynamos that started with a weak and small-scaled magnetic field saturate at a multipolar and non-axisymmetric magnetic field, while the dynamos that started with a magnetic field that had a strong axial dipole saturate in a dipole-dominant field configuration. We used either of these magnetic field 
configurations as an initial condition to explore different dynamo solutions for a given set of control parameters.

\subsubsection{Numerical technique}

The model equations are solved using the MHD-code MagIC (Wicht 2002, Gastine and Wicht 2012), which has been tested using community-based benchmark simulations (Jones et al. 2011). After decomposing the mass flux and magnetic field into toroidal and poloidal components as

$$
\begin{aligned}
\tilde{\rho} \mathbf{u} & =\nabla \times(\nabla \times W \hat{r})+\nabla \times X \hat{r}, \\
\mathbf{B} & =\nabla \times(\nabla \times Y \hat{r})+\nabla \times Z \hat{r},
\end{aligned}
$$

the scalar potentials $W, X, Y, Z$, the pressure, and the entropy are then expressed in terms of spherical harmonics in longitude and latitude and Chebyshev polynomials in radius. The system of equations is time-advanced using an explicit second-order Adams-Bashforth scheme for Coriolis and non-linear terms and an implicit Cranck-Nicolson scheme for the remaining terms (Glatzmaier 1984, Christensen and Wicht 2007).

\subsubsection{Control parameters}

Capturing the dynamics of rotationally dominated large-scale convection in stellar interiors is the primary aim of this study, and the control parameters we chose reflect this to some extent. However, the technical feasibility severely constrains our control parameter choice. Hence, this study, or any global numerical study, for that matter, implicitly assumes that the unresolved small-scale turbulence does not affect the large-scale properties of the system. The control parameters for different setups are listed in Table 5.1.

There have been some unsuccessful attempts at generating an axial-dipole dominated (ADD) magnetic field in global numerical simulations with density-stratified convection zones (Dobler et al. 2006, Browning 2008). This is in stark contrast with the studies of planetary dynamos (which usually ignore density stratification), where ADD solutions are found frequently (Jones 2011). The planetary dynamo simulations (e.g. Christensen and Aubert 2006) persistently show that as the Ekman number, which quantifies the importance of viscous effects as compared to rotational effects, is decreased (i.e. increasing rotational influence), ADD magnetic fields become more stable and can be obtained at low values of $P m$. For example, ADD fields have been found at $P m$ as low as 0.06 for $E \approx 10^{-6}$ in geodynamo simulations (Christensen and Aubert 2006). Reaching such a small $E$ in anelastic simulations would be a much more demanding task.

Systematic investigations have revealed that as the density stratification increases in the convection zone, ADD dynamos gradually become unstable (Gastine et al. 2012, Schrinner et al. 2014). Schrinner et al. (2014) showed that for moderate Ekman numbers used in density-stratified simulations a high $\mathrm{Pm}$ might be favourable for attaining ADD dynamos. Decreasing the amplitude of differential rotation (in the form of prograde equatorial jets that are typically found in simulations) might also help to stabilize ADD dynamos (Duarte et al. 2013, Schrinner et al. 2014). The Prandtl number has been shown to affect the amplitude of the equatorial differential rotation (Christensen 2002, Simitev 


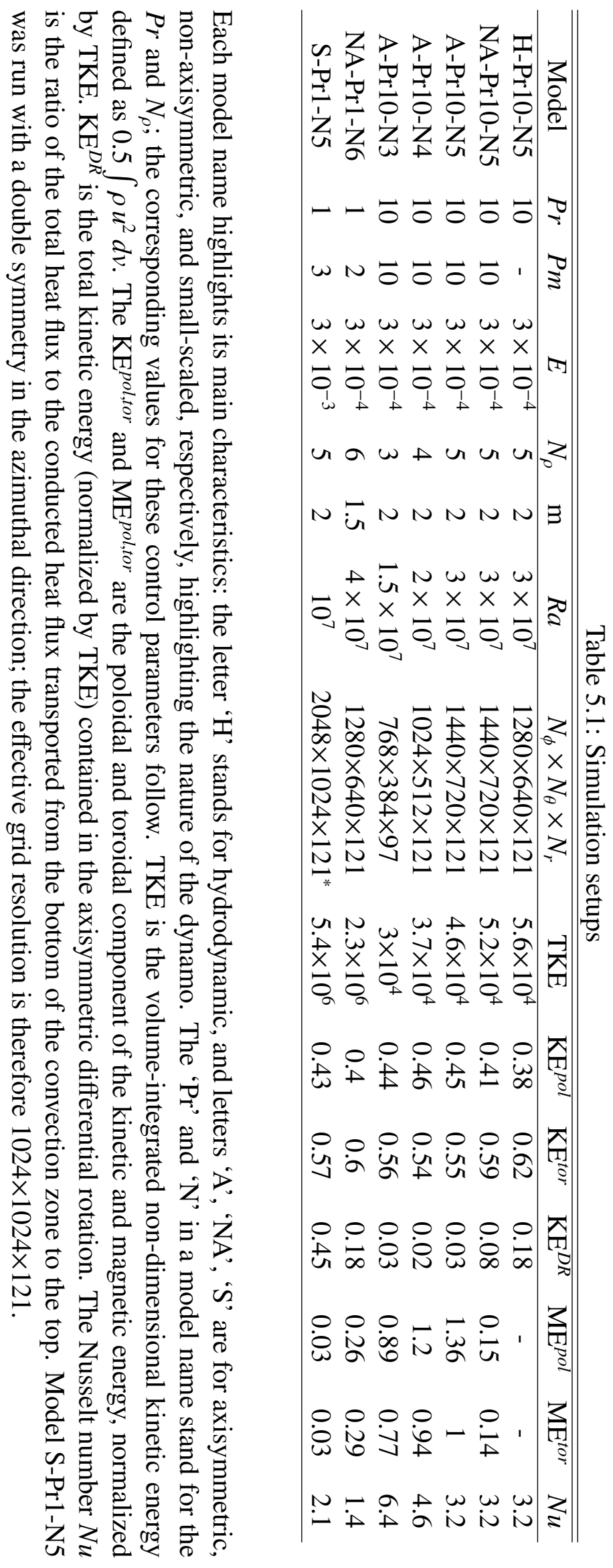


and Busse 2005). Keeping these results in mind, we used relatively high values of $\operatorname{Pr}$ and $P m$ in our simulations (see Table 5.1). Moreover, simulations with $\operatorname{Pr}$ of 10 generate convection with moderate Reynolds numbers $R e=v d / v$ ( $v$ is some appropriately averaged velocity). Consequently, a relatively high value of $P m$ is required in these simulations to attain a high enough magnetic Reynolds number $R m=R e P m$ for dynamo action to occur. The Ekman number is set to $3 \times 10^{-4}$ (except for S-Pr1-N5). This is equivalent to a Taylor number $\left(=4 / E^{2}\right)$ of about $4 \times 10^{9}$. This value allowed us to carry out a small parameter study in a manageable time frame while still having rotationally dominated convection in a medium with strong density stratification.

Simulations with density-stratified convection zones have relatively slower and largescaled flow in high-density regions while the flow is rapid and has smaller scales in regions of low density. The latter demands smaller time-steps and higher grid resolution. Furthermore, to ensure that a dynamo solution is in an equilibrium state, the simulation should run for at least a magnetic diffusion time, implying a longer run time at higher Pm. Within these constraints, the highest density contrast we could afford in simulations with $P m=10$ was about 150. The polytropic index also had to be increased to 2 (from a more appropriate value of 1.5 for a monoatomic ideal gas) in these cases to avoid a steep drop in density in the outer layers, which would require a higher grid resolution to resolve. For a case with $P m=2$, however, we were able to reach a density contrast of about 400 with a polytropic index of 1.5. An exception was made for model S-Pr1-N5, which was run for a third of the magnetic diffusion time because of the severe computational requirements.

\subsubsection{Numerical grid resolution}

All the simulations reported in this study were first run on a grid with $768 \times 384 \times 121$ grid points, where the three numbers represent the grid resolution in longitude, latitude, and radius, respectively. This grid was sufficient to adequately resolve the flow and magnetic field in most of the interior of the shell, but the relatively vigorous convection near the surface remained under-resolved in most cases. We then stepwise increased the grid resolution and ran the simulation long enough to confirm the results. The resolution reported in Table 5.1 is the highest resolution used for a particular simulation.

\subsection{Results}

We begin by discussing the results of a simulation for purely hydrodynamical convection and later explore its dynamo action.

\subsubsection{Hydrodynamical convection}

To roughly assess how rapid rotation influences convection, we used the so-called convective Rossby number $R o_{c}=\sqrt{R a E^{2} / \operatorname{Pr}}$ (introduced by Gilman 1977), which estimates the ratio of buoyancy to Coriolis forces. For $R o_{c} \ll 1$, rotational effects dominate. The model H-Pr10-N5 (see Table 5.1) has $R o_{c} \approx 0.5$. This means that here convection is probably substantially influenced by rotation. Note that $R o_{c}$ can be decreased either by 

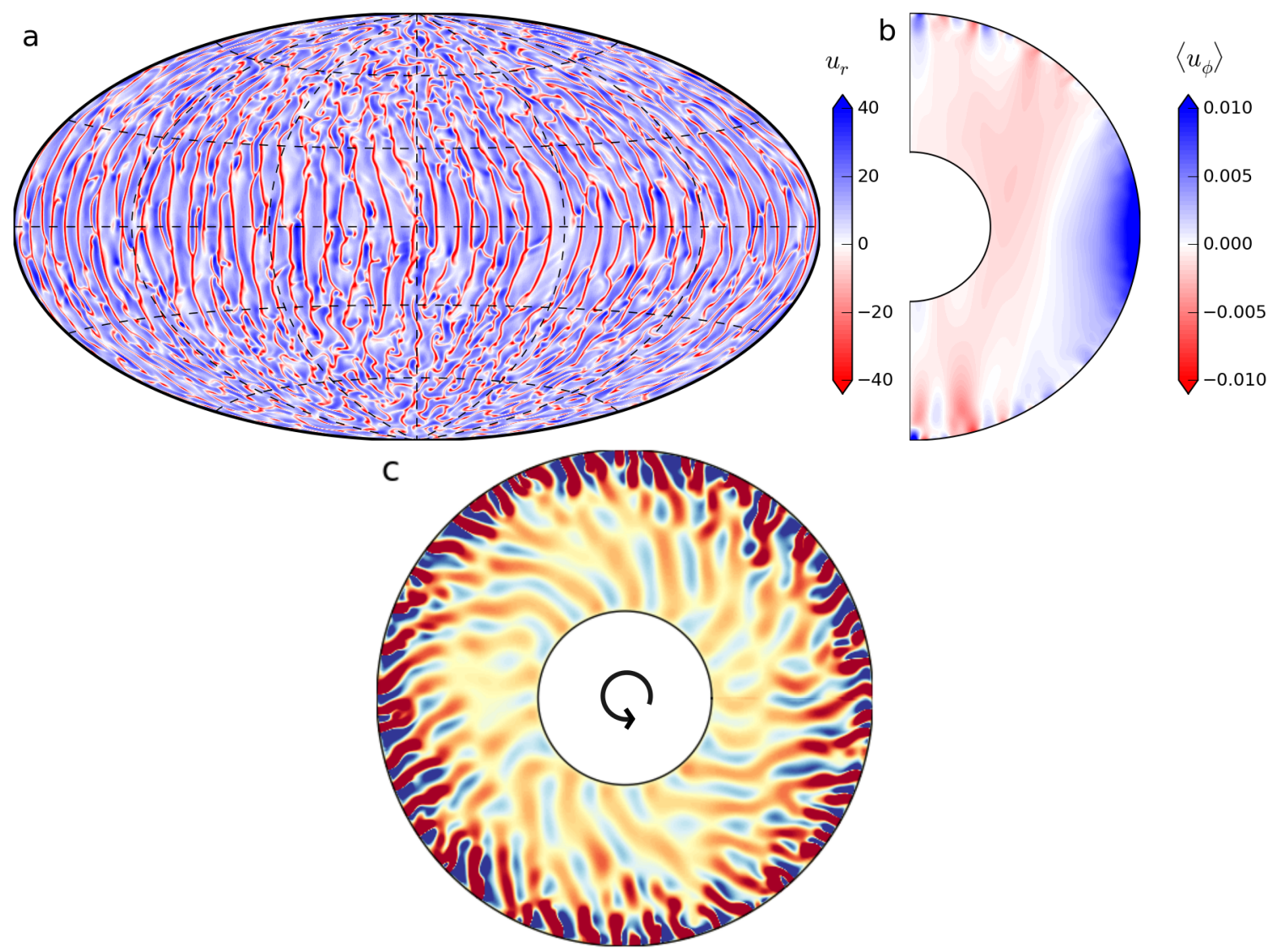

Figure 5.1: Flow patterns for hydrodynamical model H-Pr10-N5. Hammer projection of radial velocity $u_{r}$ at $r=0.99 r_{o}$ is displayed in (a), while the longitudinally averaged azimuthal velocity $\left\langle u_{\phi}\right\rangle_{\phi}$ (or differential rotation) is given in (b). Red and blue indicate a downwelling and an upwelling flow in (a) and a westward and an eastward flow in (b). $u_{r}$ and $\left\langle u_{\phi}\right\rangle_{\phi}$ are given in terms of the Reynolds number $R e=u d / v$ and the Rossby number $R o=u /(d \Omega)$. The $\mathrm{z}$ component of vorticity is shown in $(\mathbf{c})$, where red and blue shades represent positive and negative vorticity. The colour variations are saturated at values lower than the extrema.

decreasing $E$ (computationally very demanding) or by increasing $\operatorname{Pr}$. The last scenario has been exploited in setting the control parameters for this and most other cases.

Various quantities describing the convection patterns of model $\mathrm{H}-\mathrm{Pr} 10-\mathrm{N} 5$ are portrayed in Fig. 5.1. The radial velocity $u_{r}$ near the outer boundary shown in (a) highlights the typical broad upwellings and narrow downwellings that are formed by the influence of density stratification. The flow in the deep interior, however, consists of large-scale helical columns aligned with the rotation axis, as seen in a 3D rendering (not shown for this case). This convection pattern is typical for rotationally dominated convection in simulations of density-stratified convection zones (Miesch 2005, Browning 2008, Gastine and Wicht 2012). The structural change arises because the influence of rotation varies as a function of radius in a density-stratified fluid and is weaker in the outer layers (Gastine and Wicht 2012). In (b) the axisymmetric longitudinal velocity $\left\langle u_{\phi}\right\rangle_{\phi}$ (angle brackets $\langle\ldots\rangle_{x}$ represents averaging over $x$ ) or the differential rotation varies only moderately on 


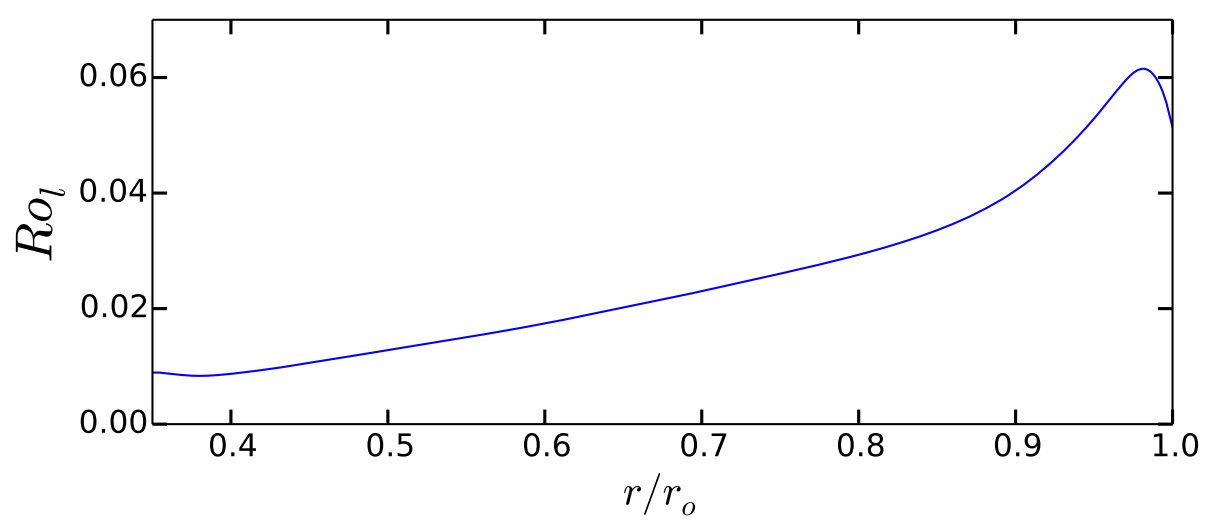

Figure 5.2: Variation of the local Rossby number $R o_{l}$ as a function of radius for the hydrodynamical model H-Pr10-N5. $u$ and $l$ have been averaged over a few rotation periods.

cylinders aligned with the rotation axis, except near the outer boundary. The typical differential rotation profile, that is, faster equator and slower poles, is maintained by the Reynolds stresses, which arise because of the statistical correlation between radial and longitudinal flow components (Christensen 2002, Simitev and Busse 2005). Reynolds stresses are promoted by the spiralling nature of convection columns that are tilted in the direction of the shell rotation (Gilman 1975, Busse 1983). The plot of the axial fluid vorticity $\omega_{z}=(\nabla \times \mathbf{u})_{z}$ in the equatorial plane of the shell in (c) shows the spiralling columns.

A more refined estimate for the rotational influence can be obtained via the local Rossby number (Christensen and Aubert 2006), defined here as a function of radius by $R o_{l}=\langle u\rangle_{\theta, \phi, t} /(\Omega l)$ with the longitudinally, latitudinally, and time-averaged velocity $\langle u\rangle_{\theta, \phi, t}$. The length scale $l=\pi / \bar{\ell}_{u}$ is a characteristic length scale of the flow at radius $r$ defined using

$$
\bar{\ell}_{u}=\sum_{\ell} \frac{\ell\left\langle\mathbf{u}_{\ell} \cdot \mathbf{u}_{\ell}\right\rangle_{\theta, \phi}}{\langle\mathbf{u} \cdot \mathbf{u}\rangle_{\theta, \phi}}
$$

where $\mathbf{u}_{\ell}$ is the flow component at a given radius for spherical harmonic degree $\ell$. The radial variation of $R o_{l}$ for model H-Pr10-N5 is shown in Fig. 5.2. Previous parameter studies have shown that as long as $R o_{l}$ is smaller than a threshold value of $\approx 0.1$, Coriolis forces significantly affect the nature of convection (Christensen and Aubert 2006, Schrinner et al. 2012, Gastine et al. 2013b, 2014). Here, $R o_{l}<0.1$ in the entire shell. This explains why Fig. 5.1(a) shows north-south aligned convection cells even near the outer boundary of the simulation.

\subsubsection{Self-consistent dynamos}

\subsubsection{Model NA-Pr10-N5}

We now consider the dynamo action of the case discussed above. We used the hydrodynamic model H-Pr10-N5 as starting point and set $P m=10$, which results in model NAPr10-N5. A very weak seed magnetic field was exponentially enhanced by the helical 

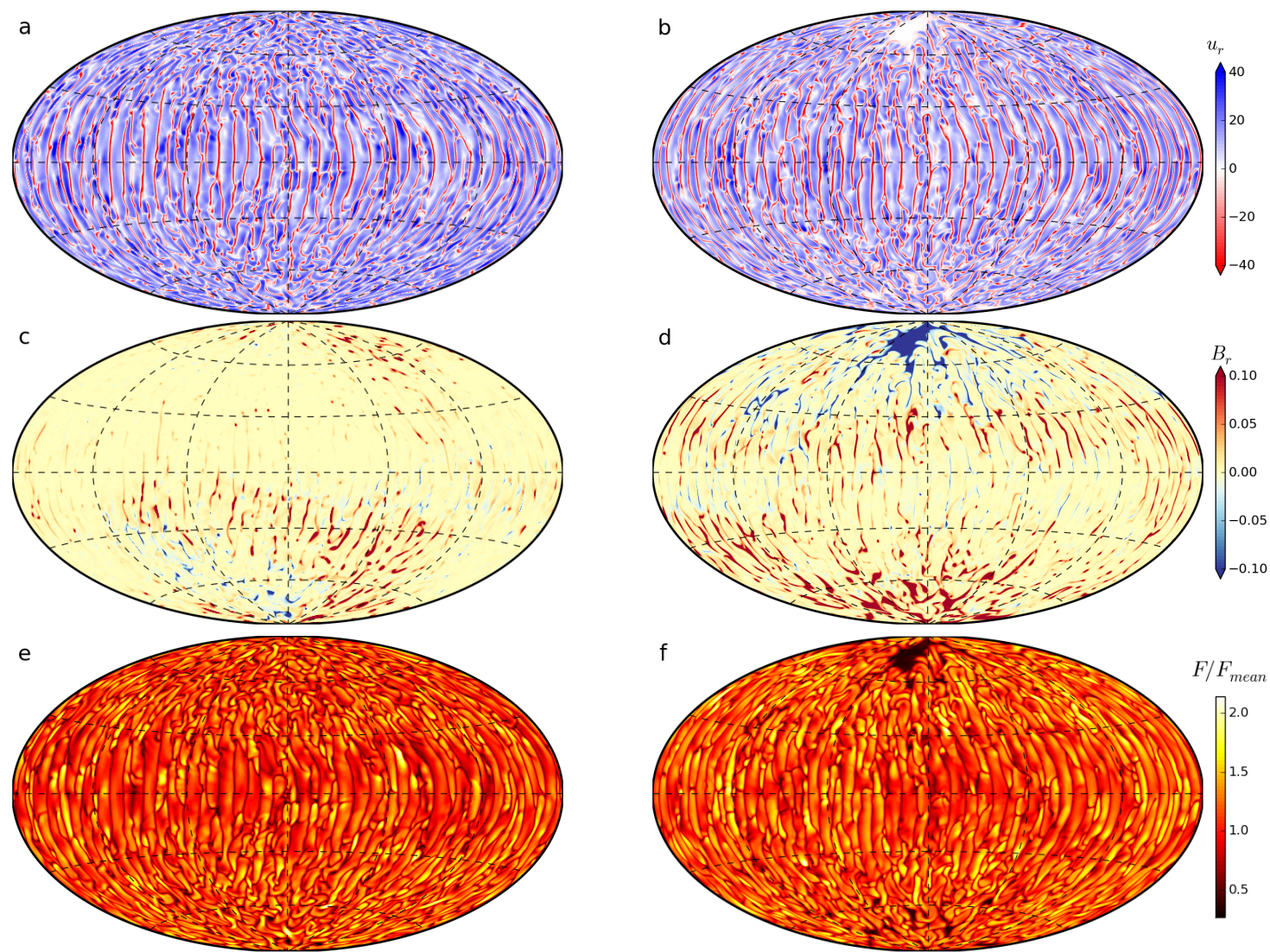

Figure 5.3: Radial velocity $u_{r}$ at $r=0.99 r_{o}$ in (a), (b), radial magnetic field $B_{r}$ at $r=0.99 r_{o}$ in (c), (d), and total heat flux $F$ normalized by its surface mean $F_{\text {mean }}$ on the outer surface in (e), (f) for models NA-Pr10-N5 and A-Pr10-N5. $u_{r}$ is given in terms of the Reynolds number, and the radial magnetic field is normalized by the equipartition field strength calculated using the time-averaged total kinetic energy in the shell. The colour variations for $u_{r}$ and $B_{r}$ are saturated at values lower than the extrema; the full variation range for $B_{r}$ is $\approx \pm 0.3$ in $(\mathbf{d})$.

convection, and the system finally saturated to a statistically stationary state. A snapshot of the simulation in the saturated regime showing the radial velocity and the radial magnetic field near the outer boundary is shown in Fig. 5.3(a) and (c). The dynamo-generated magnetic field is non-axisymmetric, and the morphology is dominated by a spherical harmonic order $m=1$ component. The magnetic field at the instant shown in Fig. 5.3(c) is also concentrated in the southern hemisphere. However, the hemisphere with the dominant magnetic field can change with time (Grote and Busse 2000). The field also propagates westwards in the rotating frame of reference of the shell. A "butterfly" diagram (not shown) of the azimuthally averaged radial field also shows poleward-propagating features. Such travelling non-axisymmetric dynamo solutions have previously been observed in dynamos with free-slip boundary conditions (Schrinner et al. 2011, 2012, Käpylä et al. 2013) and can be described in terms of the classical Parker-waves (Busse and Simitev 2006, Goudard and Dormy 2008, Schrinner et al. 2012).

As shown in Fig. 5.3(c), the diverging upwellings sweep the magnetic flux and concen- 
trate it into the convergent downwellings. This sort of redistribution of magnetic flux is a generic trait in magnetic convection (Proctor and Weiss 1982, Vögler et al. 2005, Stein and Nordlund 2006, Stein 2012). Furthermore, the expelled magnetic flux preferably accumulates into the nodes of the downwelling lanes of convection cells (Stein 2012).

In Fig. 5.3(e) we plot the total heat flux normalized by its surface-averaged value on the outer boundary of the simulation. The heat flux at the outer boundary is calculated as $F=-\operatorname{Pr}^{-1} \tilde{\rho} \tilde{T}(d s / d r)$, where $\tilde{\rho}$ and $\tilde{T}$ are the local (background) density and temperature and $d s / d r$ is the local radial derivative of entropy. Comparing Fig. 5.3(a) with Fig. 5.1(a) shows that the radial flow is very similar in both cases. Nonetheless, a careful inspection of the nodes of the downwelling lanes does show a quenching of radial flow where the magnetic flux is concentrated. Correspondingly, the convective heat flux is also reduced in these regions (e.g. the tiny magnetic flux patch near the south pole in Fig. 5.3(c)).

Compared with model H-Pr10-N5, the differential rotation (Fig. 5.4(a)) is quenched and the eastward tilt of helical convection columns (Fig. 5.4(b)) is reduced by the magnetic field in this case. The energy content of the axisymmetric differential rotation for H-Pr10-N5 and NA-Pr10-N5 is about $18 \%$ and $8 \%$ of the total kinetic energy (Table 5.1). The qualitative structure of differential rotation, however, is similar in both cases (see Fig. 5.1(b) and Fig. 5.4(a)). The total magnetic energy of model NA-Pr10-N5 is only about $30 \%$ of the total kinetic energy (see Table 5.1 and Fig. 5.5(a)). In the mean-field formulation, such non-axisymmetric multipolar dynamos can be categorized as $\alpha \Omega$ or $\alpha^{2} \Omega$ type (Schrinner et al. 2012, Gastine et al. 2012), where magnetic field co-exists with substantial differential rotation. Dynamo simulations resembling this case (i.e. cases with significant density stratification and a multipolar magnetic field) have been reported frequently (Miesch 2005, Browning 2008, Ghizaru et al. 2010, Käpylä et al. 2012, Gastine et al. 2012, Schrinner et al. 2012, Nelson et al. 2013, Fan and Fang 2014, Cole et al. 2014) and do not exhibit any prominent low heat flux regions linked to strong magnetic fields that could be associated with starspots (see Fig. 5.3(e)).

\subsubsection{Model A-Pr10-N5: Polar starspots}

Instead of initiating the dynamo simulation with a tiny seed magnetic field, we now took the flow input from model H-Pr10-N5 and initiated the dynamo simulation with a strong dipolar magnetic field aligned with the rotation axis. The resulting dynamo model is A-Pr10-N5 (Table 5.1). The motivation behind starting with a strong dipolar field is to have strong Lorentz forces that can quench the shearing differential-rotation via Maxwell stresses (Ferraro 1937, Aubert 2005). This allows an axial-dipole dominant (ADD) solution to develop and stabilize. As shown in Fig. 5.4(c), the axisymmetric differential rotation becomes even more strongly quenched (energy content of about $3 \%$ of the total kinetic energy) compared with the non-magnetic model than for NA-Pr10-N5 and and has entirely lost any semblance to geostrophy. Reynolds stresses are not effective any more because the spiralling of convection columns nearly vanishes (Fig. 5.4(d)). Such a differential rotation profile is typically associated with a thermal wind balance (Aubert 2005), that is, a balance of buoyancy and Coriolis forces. This ADD solution is stable because we ran this simulation for about two magnetic diffusion times $\left(d^{2} / \lambda\right)$. This ADD configuration would have decayed after a magnetic diffusion time if it had not been selfconsistently sustained by the convection. Note that one magnetic diffusion time is equal to 

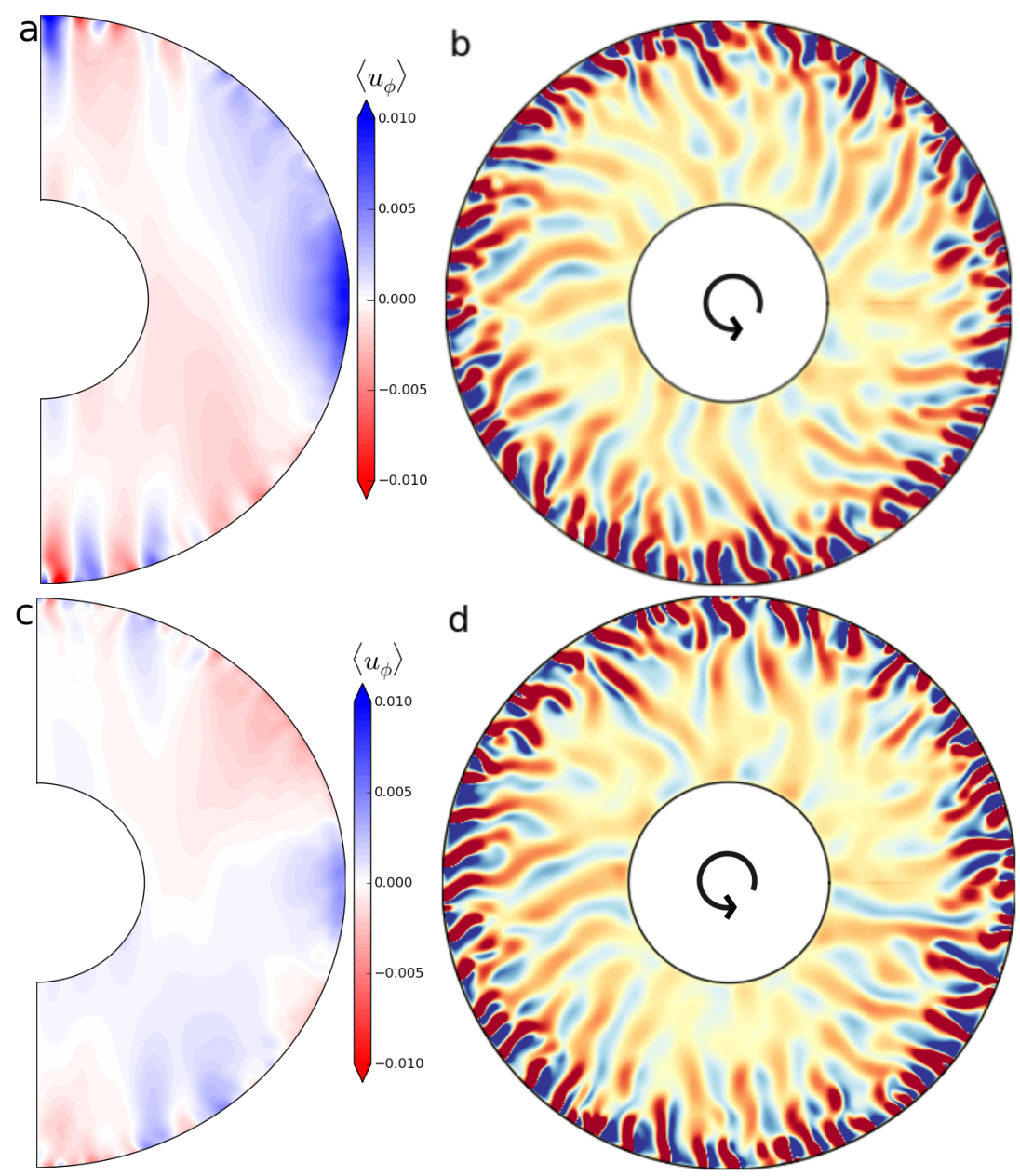

Figure 5.4: Longitudinally averaged azimuthal flow in (a) and (c) and $\omega_{z}$ in (b) and (d) for model NA-Pr10-N5 and A-Pr10-N5.

one thermal diffusion time $\left(d^{2} / \kappa\right)$ since both $P m$ and $P r$ are equal for this case. Therefore, the simulation is also thermally relaxed.

Dynamos with an ADD magnetic field that co-exist with highly quenched differential rotation are classified as $\alpha^{2}$-dynamos (Olson et al. 1999, Chabrier and Küker 2006, Schrinner et al. 2012). Dynamos in this state are said to be in a magnetostrophic state where Lorentz forces are rather strong and enter in the first-order force balance (e.g. Sreenivasan and Jones 2006). Generally, parameter studies (Browning 2008, Gastine et al. 2012, Yadav et al. 2013b) show that large-scale magnetic fields generated by a distributed dynamo quench the differential rotation to low values (much lower than solar). Recently, this was empirically verified for a large sample of cool stars that showed a rough inverse correlation between rotation rate and differential rotation (Reinhold et al. 2013). Note that higher rotation rates generally imply stronger magnetic fields in cool stars (Pizzolato et al. 2003).

Figure 5.6 displays the radial profile of the non-dimensional luminosity $L$ calculated using the various energy fluxes defined by Eqs. (5.7-5.12). The total luminosity $L_{t o t}$ is nearly constant throughout the convection zone, signifying the statistically stationary na- 

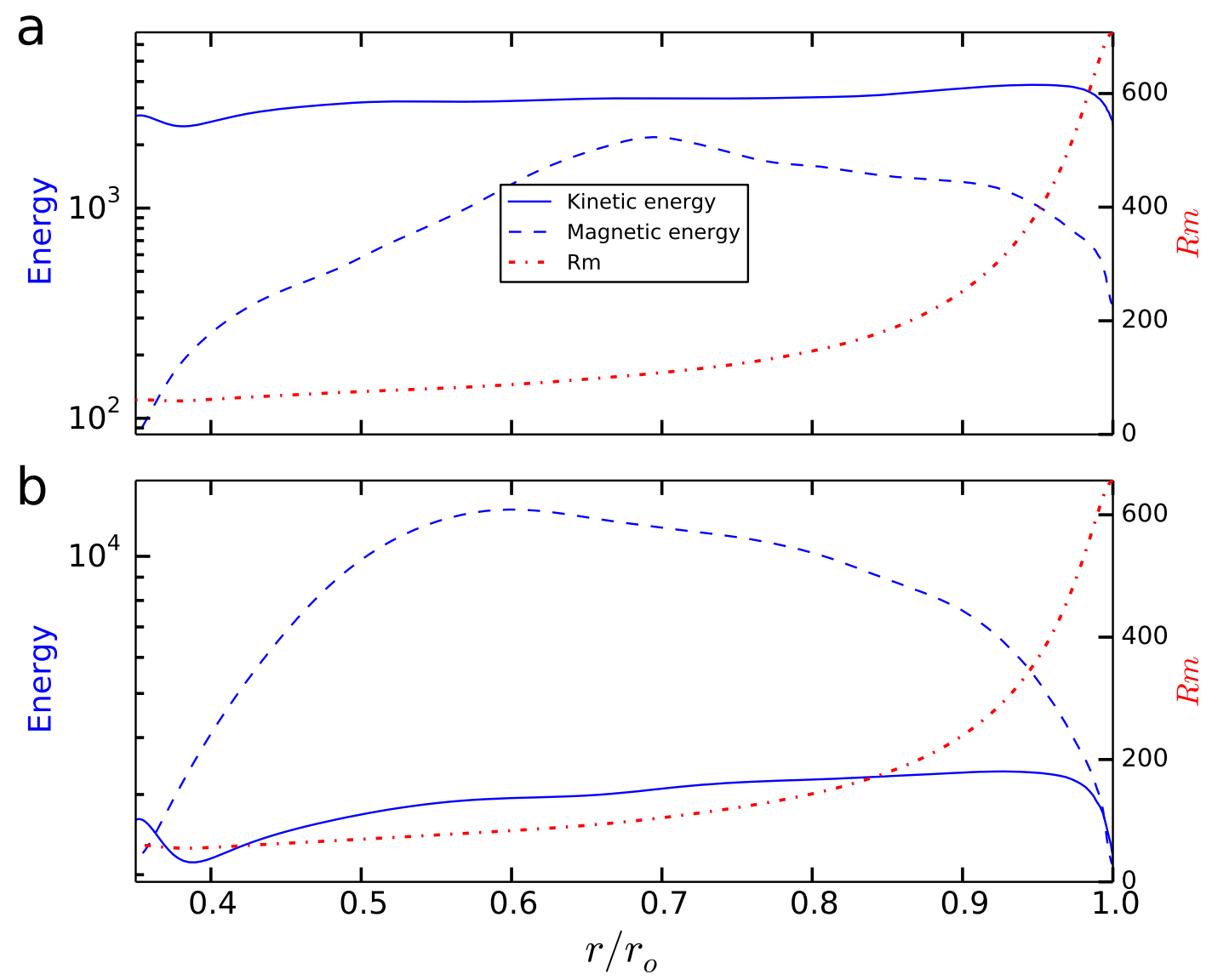

Figure 5.5: Longitudinally and latitudinally averaged kinetic energy and magnetic energy density on the left axis and magnetic Reynolds number $R m=u d / \lambda$ on the right axis as a function of radius for model NA-Pr10-N5 (panel (a)) and A-Pr10-N5 (panel (b)). The quantities were averaged over a few rotation periods.

ture of the solution. Conductive and convective contributions dominate the energy transport, with the former dominating near the boundaries, the latter in the bulk. Assuming that the thermal boundary layers (or better: entropy boundary layers, since entropy diffusion is assumed in our formulation) extend up to the point where diffusive and convective flux contributions are equal (see e.g. Julien et al. 2012), the bottom and top boundary layers span about $0.04 r_{o}$ and $0.01 r_{o}$. The thickness difference between these two boundary layers can be attributed to the high density contrast in the simulated convection zone. Viscous, Poynting, and resistive fluxes are only secondary contributions, similar to what is usually observed in such global convection simulations. Owing to the relatively small role of inertia in this dynamo model (since $\operatorname{Pr}=10$ ), the kinetic energy flux also contributes less than in earlier studies, which frequently employed $\operatorname{Pr} \leq 1$ (e.g. Miesch 2005, Nelson et al. 2013, Fan and Fang 2014).

A snapshot of the radial velocity and the radial magnetic field near the outer boundary is given in Fig. 5.3(b) and (d). Unlike the multipolar dynamo model NA-Pr10-N5, the quenching of near-surface flows in regions of strong magnetic field is rather prominent in this case and is clearly visible in Fig. 5.3(b), especially at high latitudes. The patch near 


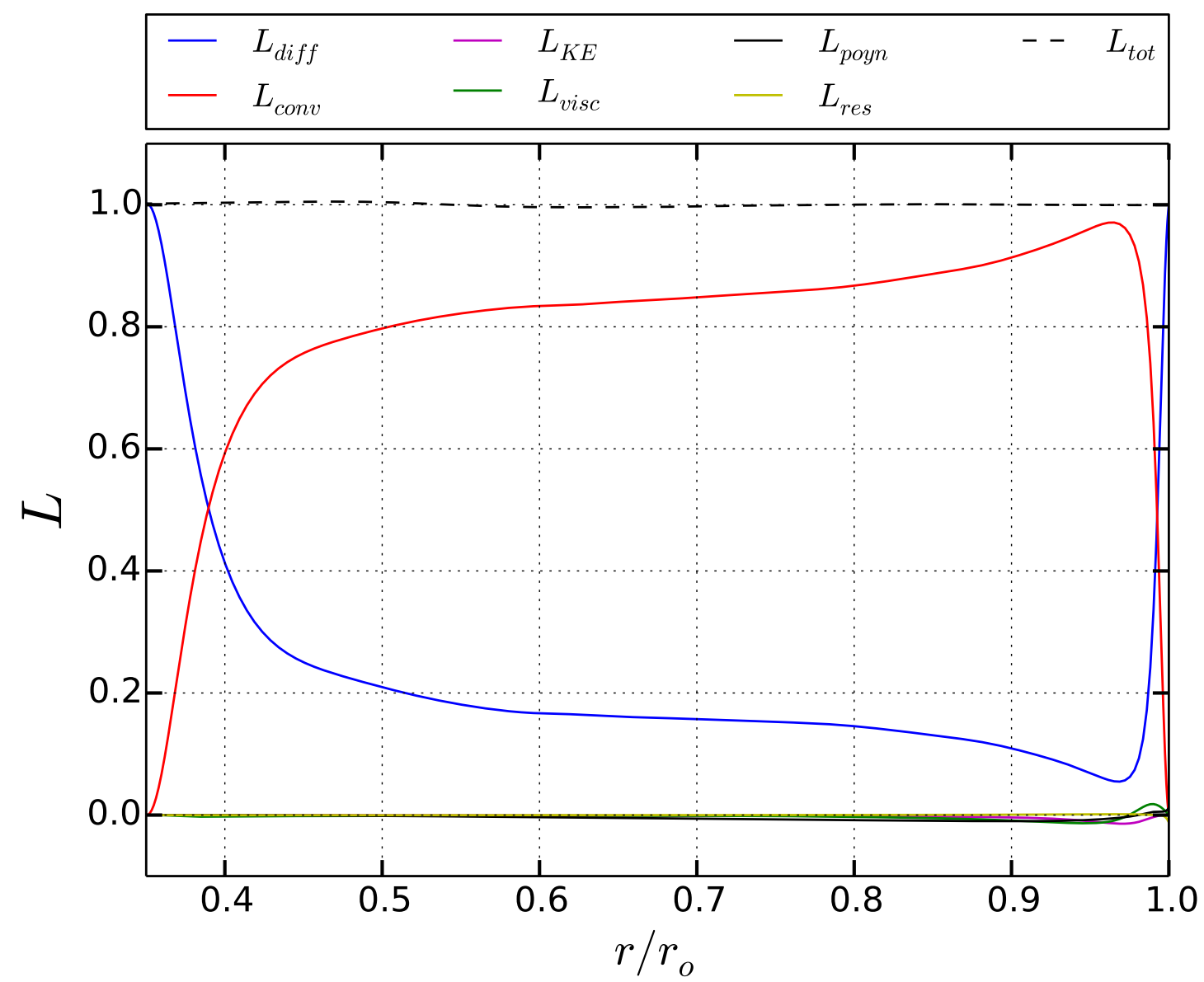

Figure 5.6: Radial profiles of non-dimensional luminosities, i.e. $r^{2} \int\langle F\rangle_{t} \sin (\theta) d \theta d \phi$, associated with the different fluxes for model A-Pr10-N5, with $L_{t o t}$ being the sum of all contributions. The different contributions are normalized by $L_{t o t}$ at $r=r_{o}$. The profiles were averaged for about 200 rotations.

the north pole with very weak radial flow is larger than the general length scale of the convection cells. At low latitudes convection forms irregular north-south aligned lanes that are associated with narrow elongated flux concentrations that are relatively shortlived. Here the field concentration is usually not strong enough to seriously impede radial flow. We use the term dark spot for a region of substantial size (similar to or larger than the local convection cells) on the surface of the model where the heat flux has been suppressed by at least $\approx 50 \%$ below the average surface heat flux. Because locally very strong magnetic fields severely quench the flow, the convective heat transport is reduced, which leads to the formation of dark spots that can be associated with cool starspots.

A comparison of the radial variation of the kinetic and magnetic energy of model APr10-N5 (Fig. 5.5(b)) with that of NA-Pr10-N5 (Fig. 5.5(a)) reveals that the magnetic energy dominates in the entire convection zone (on average) in the case with dipolar magnetic field, i.e. this case generates a super-equipartition magnetic field. Note that the production of super-equipartition fields is not novel, and geodynamo simulations frequently produce such strong magnetic fields, mimicking the scenario on Earth where 

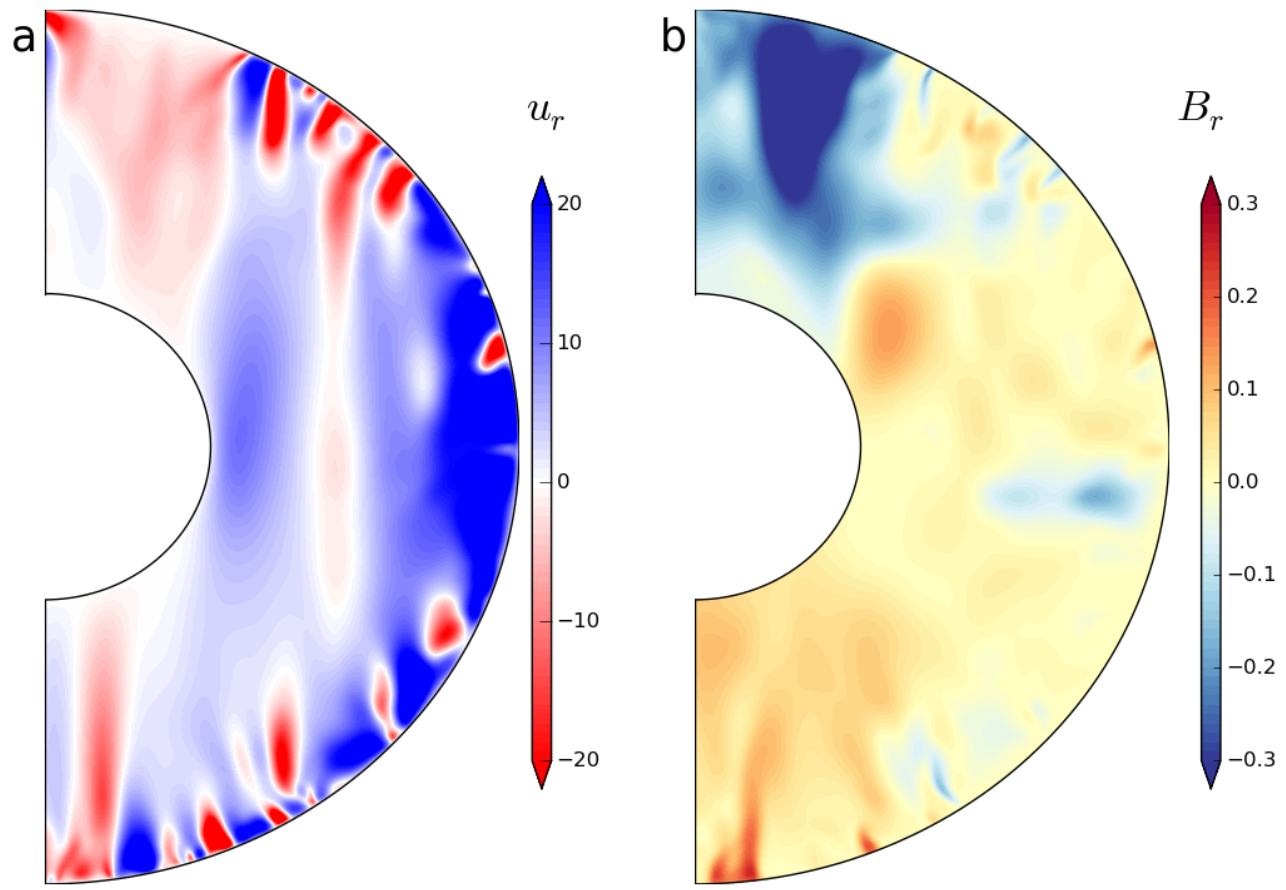

Figure 5.7: Radial velocity in (a) and radial magnetic field in (b) on a constant longitude passing through the large polar spot for model A-Pr10-N5. The colour scales saturate at values lower than the extrema.

magnetic energy is thought to be much higher than the kinetic energy (by a factor of about 7000) (Roberts and Glatzmaier 2000). In the stellar context, Featherstone et al. (2009) have reported a spherical shell dynamo with super-equipartition field strengths. Systematic numerical studies have shown that dipolar dynamos in general produce higher field strengths than the multipolar ones (Christensen 2010, Schrinner et al. 2012, Gastine et al. 2012, Yadav et al. 2013b,a).

The quenching of convective flow that leads to the formation of dark spots in this simulation is a relatively shallow phenomenon. For instance, the flow suppression at the instant shown in Fig. 5.3 is noticeable down to a depth of about $0.95 r_{o}$. However, although shallow, the quenching of convection extends well beyond the outer thermal boundary layer, which reaches down to about $0.99 r_{o}$. Figure 5.7 shows the radial velocity and the radial magnetic field on a cut along the rotation axis at a longitude passing through the large northern spot in Fig. 5.3(d). As is typical in ADD dynamos, a high concentration of magnetic flux exists at high latitudes. The magnetic field associated with this spot is seated in great depth. The integrity of this prominent flux-concentration seems to be maintained by rapid convective downwellings that surround it at its lateral margins (Fig. 5.7(a)).

\subsubsection{Time evolution of darkspots}

The attached animation, Br.mp4, shows the rich dynamics of the radial magnetic field on the outer boundary of model A-Pr10-N5. The units in the animation are similar to Fig. 5.3(f), and it spans about 75 rotations. It shows a prominent high-latitude flux- 
5 Formation of starspots in self-consistent global dynamo models: Polar spots on cool stars

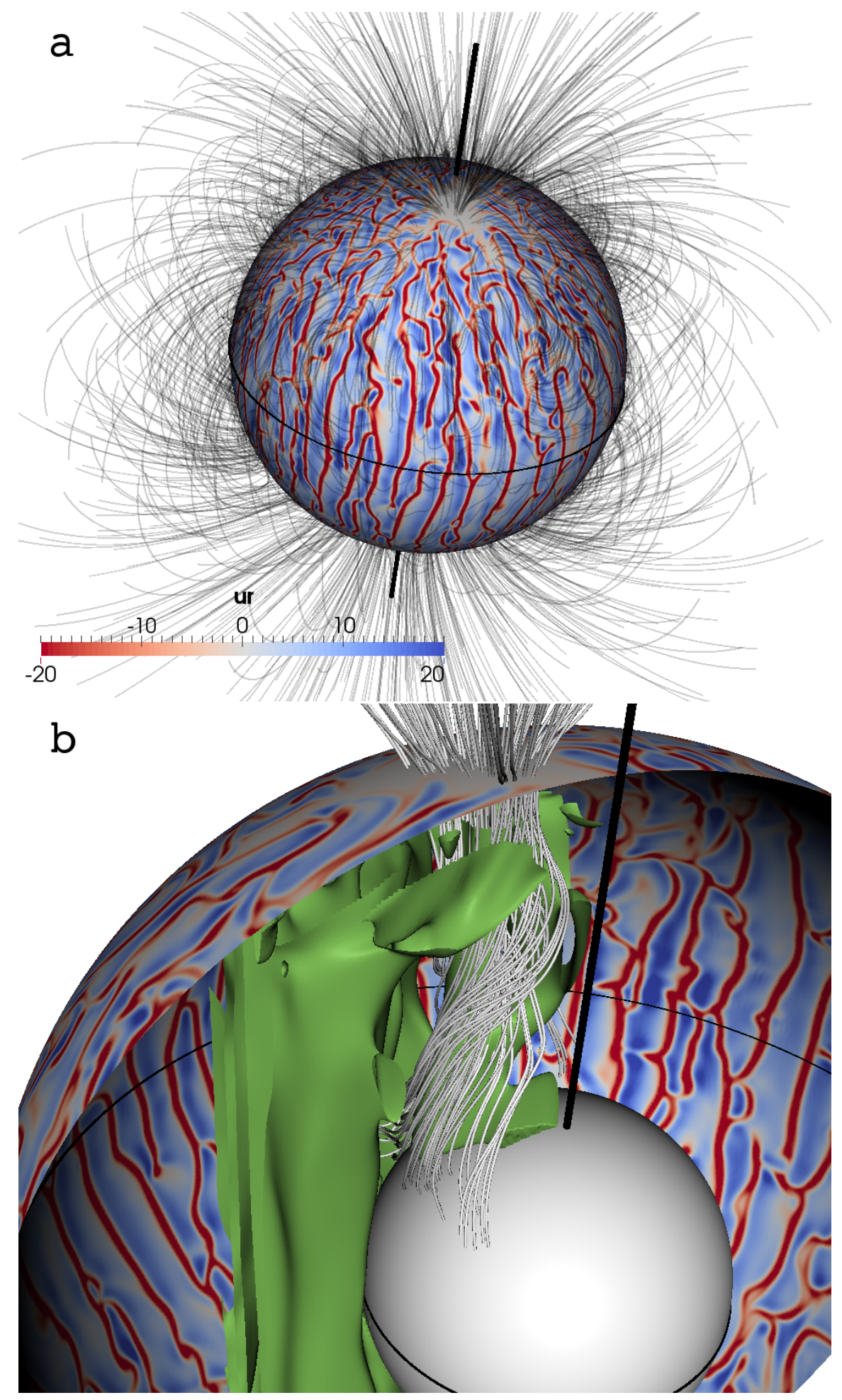

Figure 5.8: Perspective view of model A-Pr10-N5 (polar spot case) from a northern latitude. (a) radial velocity near the outer boundary and the magnetic field lines above the shell, upward continued by assuming a potential field. In (b) the surface showing the radial flow in (a) is cut and only the eastern hemisphere in shown to visualize the interior magnetic field lines associated with the northern large dark spot (visible as a white patch in (a)). The isosurface of the axial vorticity $\omega_{z}$ (in green, shown in a limited domain for clarity) is also illustrated in (b). 

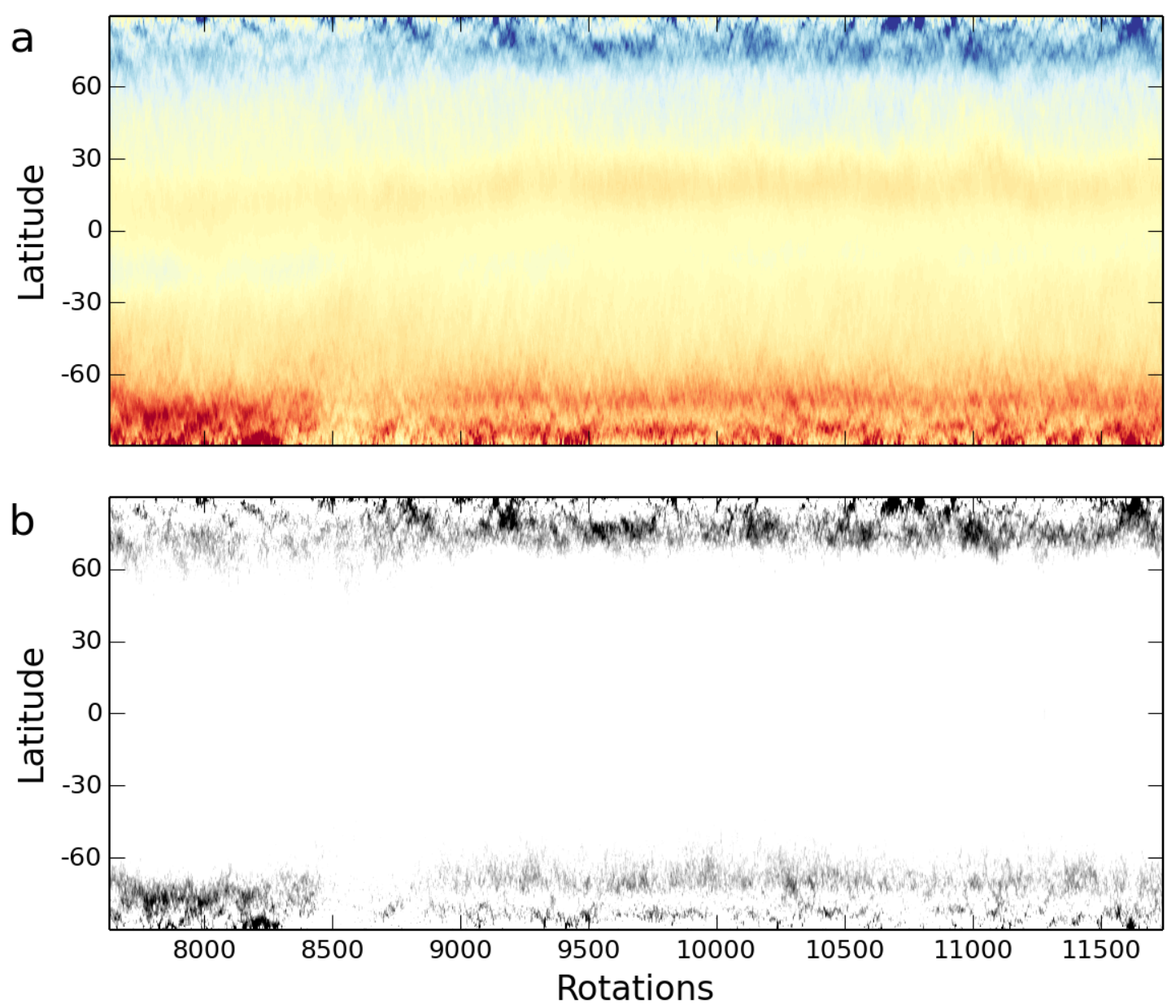

Figure 5.9: Panel (a) shows the azimuthally averaged radial magnetic field on the outer boundary of model A-Pr10-N5 (colour map similar to Fig. 5.3(d)). Panel (b) highlights magnetic-field-induced dark regions on the outer boundary. The latter were constructed by calculating the azimuthally averaged relative heat flux (sampled in Fig. 5.3(f)) for the simulation and plotting data that were lower than unity.

concentration that evolves on a much longer time scale than the local convection. Large flux-patches remain for many rotations and form when two or more sizeable patches merge. The elongated north-south-aligned convection cells near the equator act as clear pathways for tiny flux patches to migrate latitudinally.

Figure 5.9 provides a long-term perspective on the simulation by displaying azimuthally averaged radial magnetic field and relative heat flux as a function of time. Generally, the magnetic field shown in (a) is dominated by an ADD configuration. However, the dynamo solution also portrays long-term dynamics where the hemisphere with the stronger magnetic flux switches from one to the other as the simulations progresses (for instance, at around 9000 rotations). The change in the magnetic flux of a hemisphere is clearly visible in (b), which highlights the evolution of the darkest regions on the outer boundary of the simulation. Here the hemisphere with darker spots also switches from the southern 
to the northern hemisphere. Although low-latitude regions do have small magnetic-fieldinduced spots, the azimuthal averaging filters them out, which explains the absence of any feature at low latitudes.

In Fig. 5.8, we show a 3D rendering of model A-Pr10-N5. Figure 5.8(a) clearly shows the large-scale component of the magnetic fields, which is dominated by an axial dipole. Figure 5.8(b) shows that the field lines in the northern dark spot are rooted in deeper convection columns. Based on Fig. 5.8(b), we can conjecture the following formation mechanism for sizeable dark spots: first, helical columnar convection in the interior generates a collection of twisted and mainly radial magnetic field lines, and, second, the integrity of this stable magnetic structure is maintained by the downwellings of the convection in outer layers at its edges (see also Fig. 5.7). Since deep-rooted columns are wider and have a longer evolution time scale (sluggish velocity due to higher density), the flux bundles associated with them would appear as a rigid structure to near-surface convection. The dark spots formed as a result of these anchored flux bundles evolve on a time scale longer than local convection.

Observational techniques for starspot detection, such as Doppler imaging, provide only a smeared-out picture of the stellar surface. This causes the information about small scale features to be washed out. The high-latitude dark spots are commonly assumed to be a collection of smaller dark spots (Berdyugina 2005, Strassmeier 2009). To better compare our simulation results with the observational Doppler images, we smeared out the details in the simulations by filtering out all spherical harmonic degrees beyond 10 from the original simulation data. The resulting heat-flux fluctuation maps are shown in Fig. 5.10. In the non-dipolar case NA-Pr10-N5 shown in (a), the fluctuations are moderate and no consistent pattern exists. In the ADD case A-Pr10-N5 shown in (b), the dark spot in the polar region has become even more prominent. Figure 5.10 also shows other lowcontrast features on the surface that do not represent magnetic-field-driven dark spots. The attached animation LowOrder-Flux.mp4 shows the time evolution of low-order heat-flux fluctuations corresponding to animation Br.mp4. The polar spots in the north pole region maintain a broad dark feature that persists throughout the animation. Other low-contrast features in the animation near the equator and near the south pole are more dynamic.

\subsubsection{Synthetic light curves}

Synthetic light curves for the simulation with polar spots (model A-Pr10-N5) and the non-magnetic reference model H-Pr10-N5 are shown in Fig. 5.11. We calculated the light curves for one full rotation following the instance in time at which the flux is shown in Fig. 5.3. To generate the light curves, the flux at different locations was integrated for the visible hemisphere for different phase angles of rotation. Limb-darkening was incorporated by modulating the visible flux as $f(q)=f_{o}(1-w(1-\cos (q)))$, where $w$ is a limb-darkening coefficient (set to a nominal value of 0.3 ), $f(q)$ is the flux observed by the observer, $f_{o}$ is the heat flux at a location on the outer boundary of the simulation, $q$ is the angle between the radius vector to a surface point and the line of sight. The light-curve variations are qualitatively similar for different assumed inclinations of the equatorial plane with respect to the line of sight (pearled curves), however, the amplitude increases for equatorial and northern inclinations. We also calculated the light curves for the hydrodynamic simulation H-Pr10-N5; they are shown using dashed curves. These 

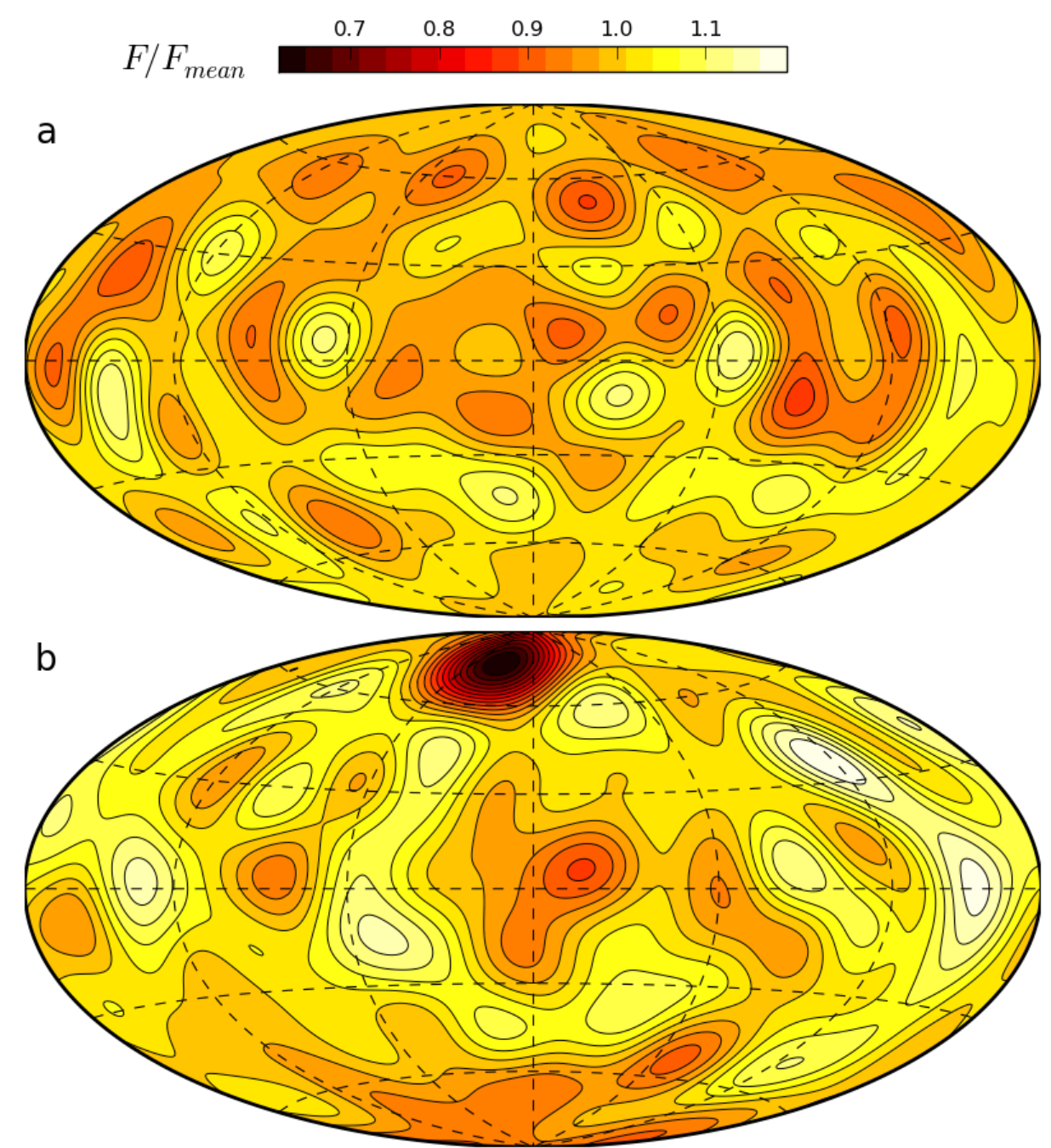

Figure 5.10: Low-order representation of Fig. 5.3(e) and Fig. 5.3(f) in (a) and (b).

hydrodynamic light curves show a variation of little more than $1 \%$. The light-curve amplitudes for the hydrodynamic model are similar to the amplitude of the southern inclination light curve of the dynamo case where the large dark spot is not visible. Hence, if we assume that the model inherently produces light-curve modulations of about $1 \%$, the large dark spot near the north pole adds an extra modulation of about $0.5 \%$. Light-curve variations of the order of a few percent are consistent with the observation of active cool stars in the Kepler data set (Basri et al. 2013).

\subsubsection{Importance of density stratification and rotation}

The results discussed above demonstrate that for a fixed density stratification and rotation rate multipolar dynamos (generating weaker field strengths) do not produce dark spots, while the ADD dynamos with stronger fields do form dark spots. Using a small parameter study, we now explore the sensitivity of dark spot formation to the density stratification 


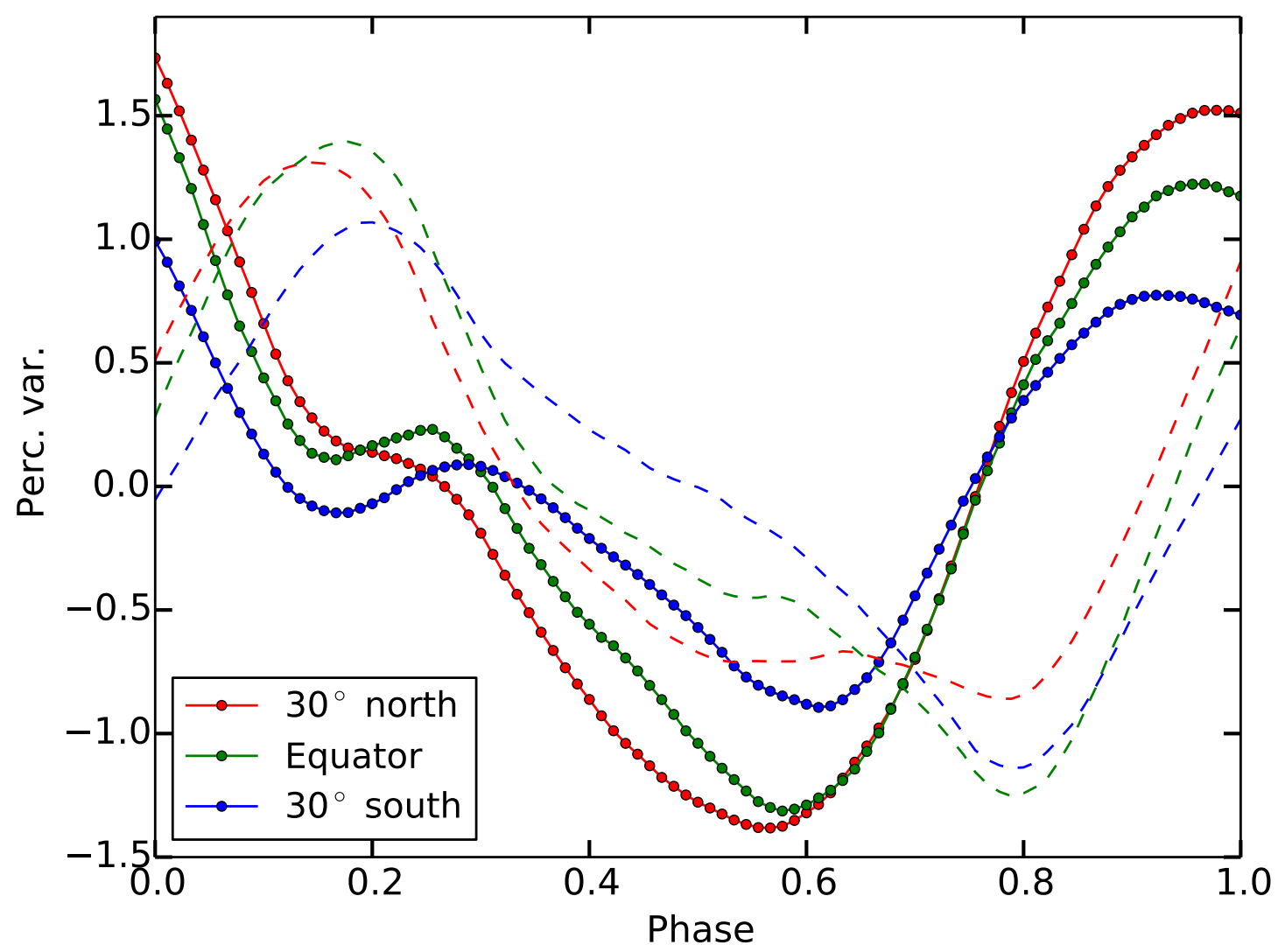

Figure 5.11: Synthetic light curves calculated using the heat flux emanating from the outer boundary of a simulation. Pearled curves depict model A-Pr10-N5, dashed curves the hydrodynamic model H-Pr10-N5. Light-curves are given for three different inclinations: line of sight $30^{\circ}$ north of the equator (red), in the equatorial plane (green), and $30^{\circ}$ south of the equator (blue). The amplitude is in terms of percentage variations about the mean.

in the convection zone and the rotation rate of the spherical shell.

We simulated two cases with a lower density stratification: model A-Pr10-N4 with $N_{\rho}=4$ (density contrast of 55) and model A-Pr10-N3 with $N_{\rho}=3$ (density contrast of 20). The entropy contrast (or equivalently $R a$ ) across the shell was changed as well, such that the simulations produced an ADD magnetic field (see Table 5.1). The resulting radial variation of kinetic and magnetic energy is shown in Fig. 5.12. As the density stratification is decreased, the magnetic energy near the outer boundary decreases sharply. Figure 5.13 displays the same quantities as Fig. 5.3 for model A-Pr10-N3. At a few inter-cellular nodes where the flux is strong enough to locally quench the flow, small dark patches are formed, but the surface is devoid of sizeable dark spots. This suggests that including a high density contrast $\left(N_{\rho} \geq 5\right)$ in the convection zone is instrumental for generating large and persistent dark spots. However, the mechanism through which density stratification promotes larger flux-concentrations is not yet clear. The proposed "negative effective magnetic pressure instability (NEMPI)" (Rogachevskii and Kleeorin 2007, Brandenburg 


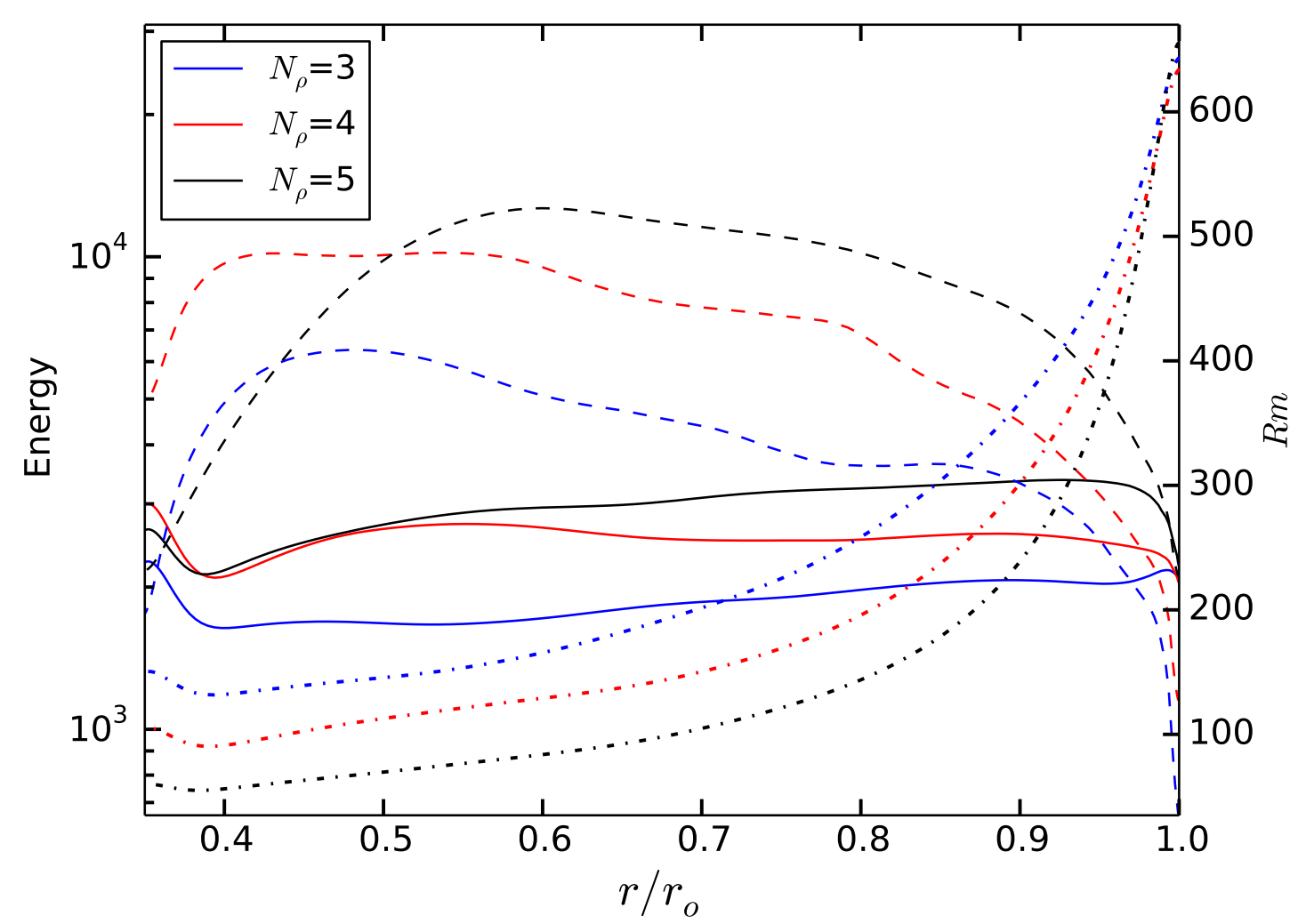

Figure 5.12: Kinetic energy $E_{k i n}$ (solid curves), magnetic energy $E_{m a g}$ (dashed curves), density on the left axis, and magnetic Reynolds number (dotted curves) on the right axis as a function of radius for three models with dipole-dominant magnetic fields, but different density stratification $N_{\rho}$ in the convection zone. Blue, red, and black curves are for models A-Pr10-N3, A-Pr10-N4, and A-Pr10-N5, respectively.

et al. 2011) also highlights the importance of density stratification for generating sizeable magnetic flux concentration. A more detailed analysis is needed to establish a connection between our simulations and the NEMPI mechanism.

Guided by the results of this discussion, we now increased the density contrast to $N_{\rho}=6$ (density contrast $\approx 400$; model NA-Pr1-N6) in the convection zone to explore whether a multipolar dynamo can form dark spots as well. $\mathrm{Pr}$ and $\mathrm{Pm}$ for NA-Pr1-N6 were lowered to avoid long saturation time scales; this came at the expense that a dominantly dipolar magnetic field could not be sustained, however (see Table 5.1). A snapshot, similar to Fig. 5.3, of dynamo model NA-Pr1-N6 is shown in Fig. 5.14. The magnetic field is dominated by a non-axisymmetric $m=1$ pattern, and it peaks in two broad longitudes in both hemispheres. This large-scale magnetic field also slowly propagates westwards, similar to model NA-Pr10-N5. The convection near the outer boundary is dominated by small convection cells, not only at high latitudes, as for A-Pr10-N5, but also near the equator. This is due to the higher $R o_{l}$ in this case, which varies from 0.05 in the interior to a maximum of about 0.25 in the outer layers. 
5 Formation of starspots in self-consistent global dynamo models: Polar spots on cool stars

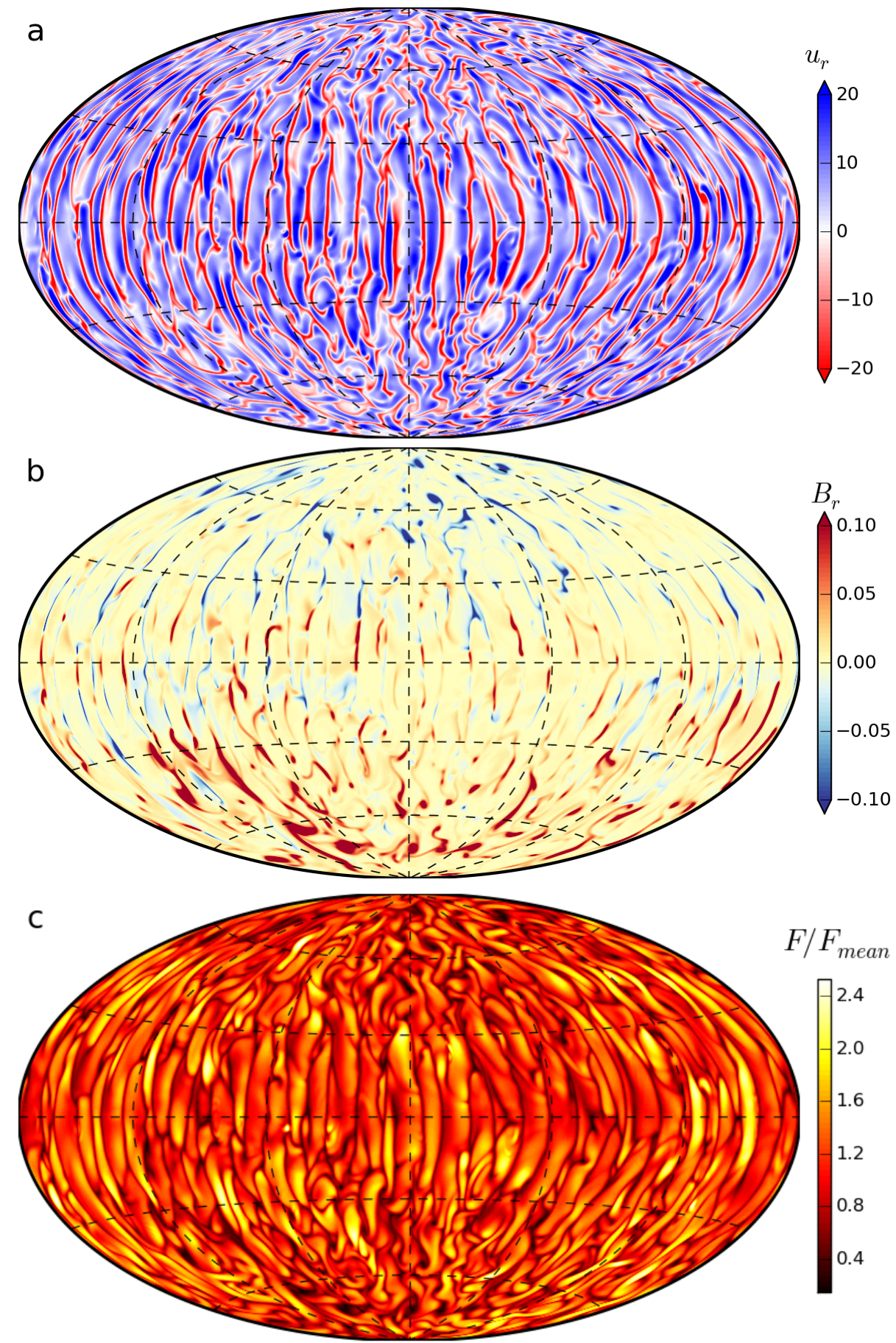

Figure 5.13: Similar to Fig. 5.3, but for model A-Pr10-N3. 
Figure 5.14(a) and (b) shows that radial velocity is quenched in many localized regions. This gives rise to dark spots that are smaller than the large spot seen in model A-Pr10-N5, but are still quite sizeable, that is, have the same scale as the convection cells. The light curve of this case, portrayed in Fig. 5.15, shows double dips that might be associated with the non-axisymmetric heat flux pattern induced by the $m=1$ magnetic field geometry. Note that the light-curve amplitudes in this case are significantly smaller than in Fig. 5.11 because the convection transports only about $30 \%$ of total heat in this case compared with about two-thirds in model A-Pr10-N5. Hence, the dark spots in this case have a lower contrast with the regions where the convection operates normally. Cole et al. (2014) have already reported similar westward-drifting magnetic fields in direct numerical simulations. Such dynamo solutions provide a rather enticing explanation for the active-longitudes phenomenon: starspots concentrated in broad preferred longitudes. Cole et al. (2014) have discussed this connection, although their simulation did not generate any dark spots. Our model NA-Pr1-N6 can be considered as a further step in this line of thought since it produces the appropriate magnetic field as well as dark spots.

The plot in Fig. 5.16 shows the radial variation of kinetic and magnetic energy density for model NA-Pr1-N6. This model is qualitatively similar to model NA-Pr10-N5 (see Fig. 5.5(a)) in that the kinetic energy dominates in this case as well. However, in outer layers, the dominance of kinetic energy is somewhat waker: the ratio of magnetic to kinetic energy density for models NA-Pr10-N5 and NA-Pr1-N6 are 0.18 and 0.25 , respectively, about $40 \%$ higher in the latter. One might speculate that the higher density stratification and weaker dominance of kinetic energy might explain why model NA-Pr1-N6 generates sizeable dark spots while model NA-Pr10-N5 does not. However, since model NA-Pr10N5 and NA-Pr1-N6 are also different in other control parameters, narrowing down the main cause for dark spot formation in the latter needs more analysis.

In another set of simulations, we maintained a strong density stratification (density contrast of $\approx 150$ ), but lowered the rotation rate (higher Ekman number) and changed other parameters so that $R o_{l}$ was of the order of one, which places it in the regime where inertia dominates rotational forces (Gastine et al. 2013a). No columnar convection exists in this case, and the flow is similar to the classical Rayleigh-Bénard convection (Fig. 5.18) and generates anti-solar differential rotation (slower equator and faster poles) (Gastine et al. 2014). The generated magnetic energy is lower than the kinetic energy by more than an order of magnitude (Fig. 5.17). As shown in Fig. 5.18(b), the magnetic field is very small-scaled and resides mainly in downwellings. No dark spots are observed in this case. Similar observations were also made by Dorch (2004) in a dynamo simulation of slowly rotating late-type giant stars. However, super-equipartition field strengths were reported in that simulation, while we observe fields with rather low strengths.

\subsection{Summary and outlook}

We have studied the spontaneous formation of dark spots in self-consistent global models of stellar convection and the associated dynamo process. We used fully non-linear anelastic simulations in rotating spherical shells. For rapid rotation, convection is in the form of large axially-aligned helical columns in the interior and more fractured and smaller scaled columns in the outer layers. A large-scale distributed dynamo operates in the bulk of the 
5 Formation of starspots in self-consistent global dynamo models: Polar spots on cool stars
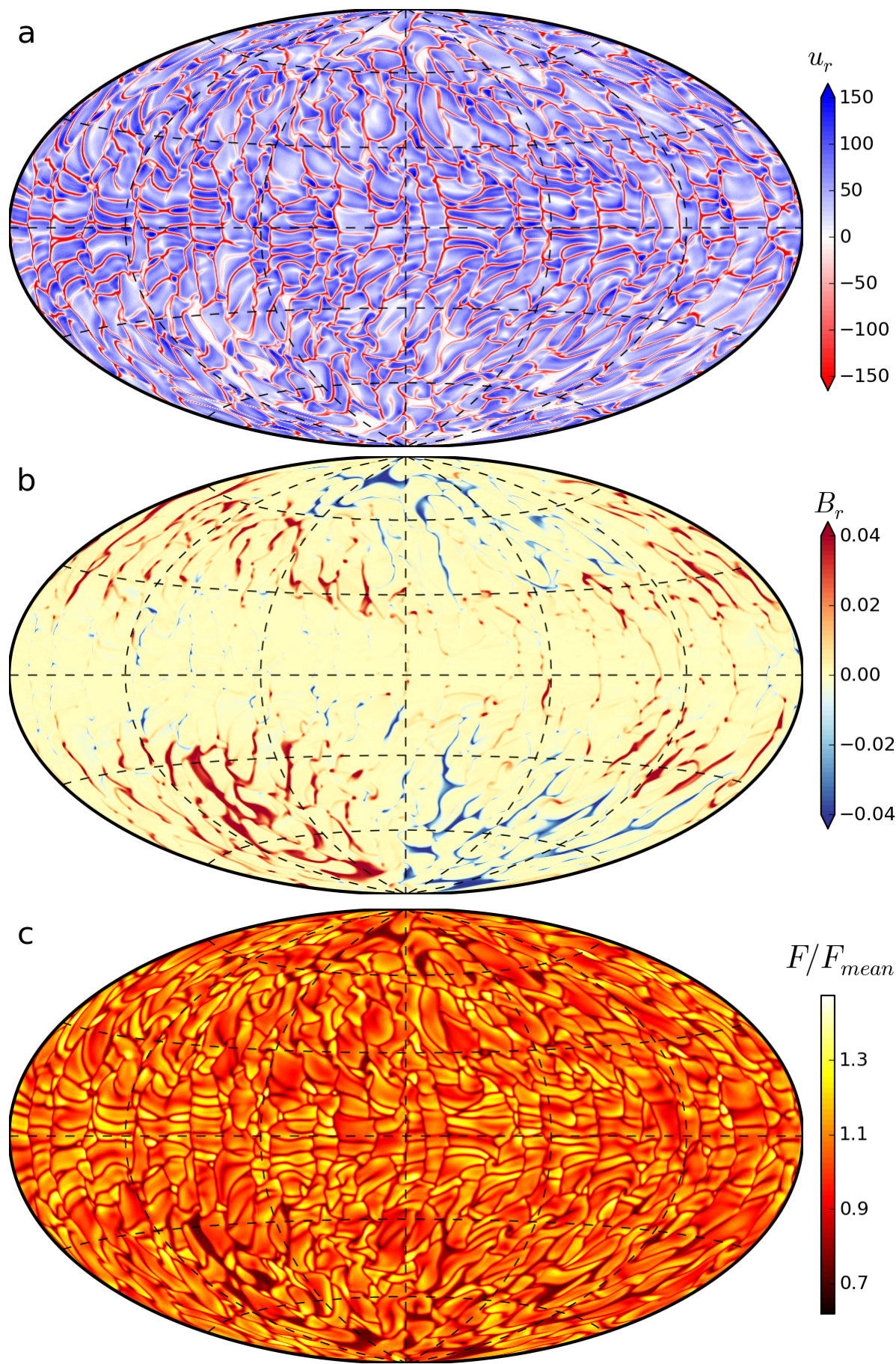

Figure 5.14: Similar to Fig. 5.3, but for model NA-Pr1-N6.

model. The dynamo generates a magnetic field that interacts with the small-scale convective motions in the outer layers where it is swept to the downwellings. This sweeping of flux leads to the formation of localized regions of intense magnetic fields. Some of these regions have a high enough field strength to locally quench convection, which leads to the formation of dark spots. 


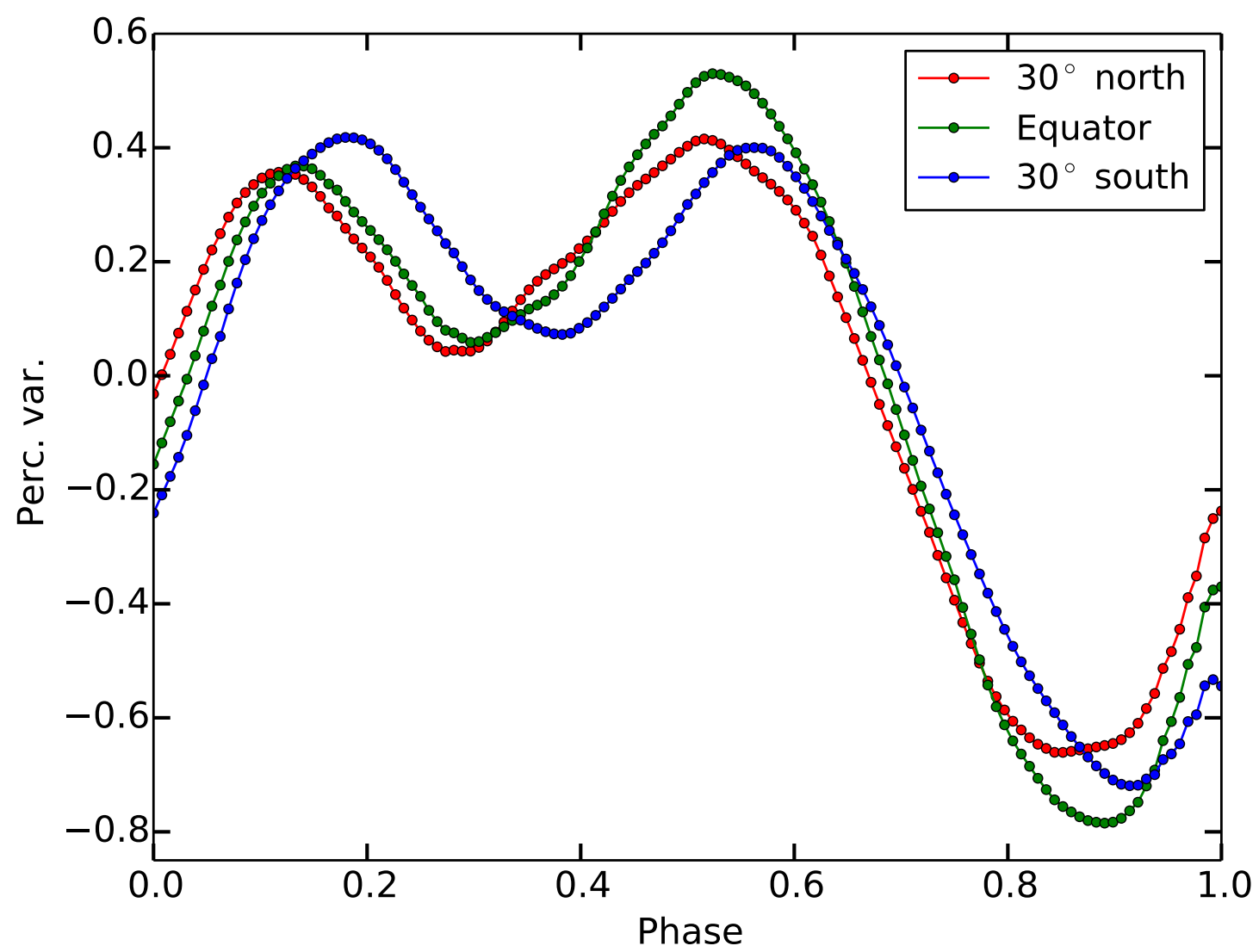

Figure 5.15: Similar to Fig. 5.11, but for model NA-Pr1-N6. Light curves are based on the instant shown in Fig. 5.14.

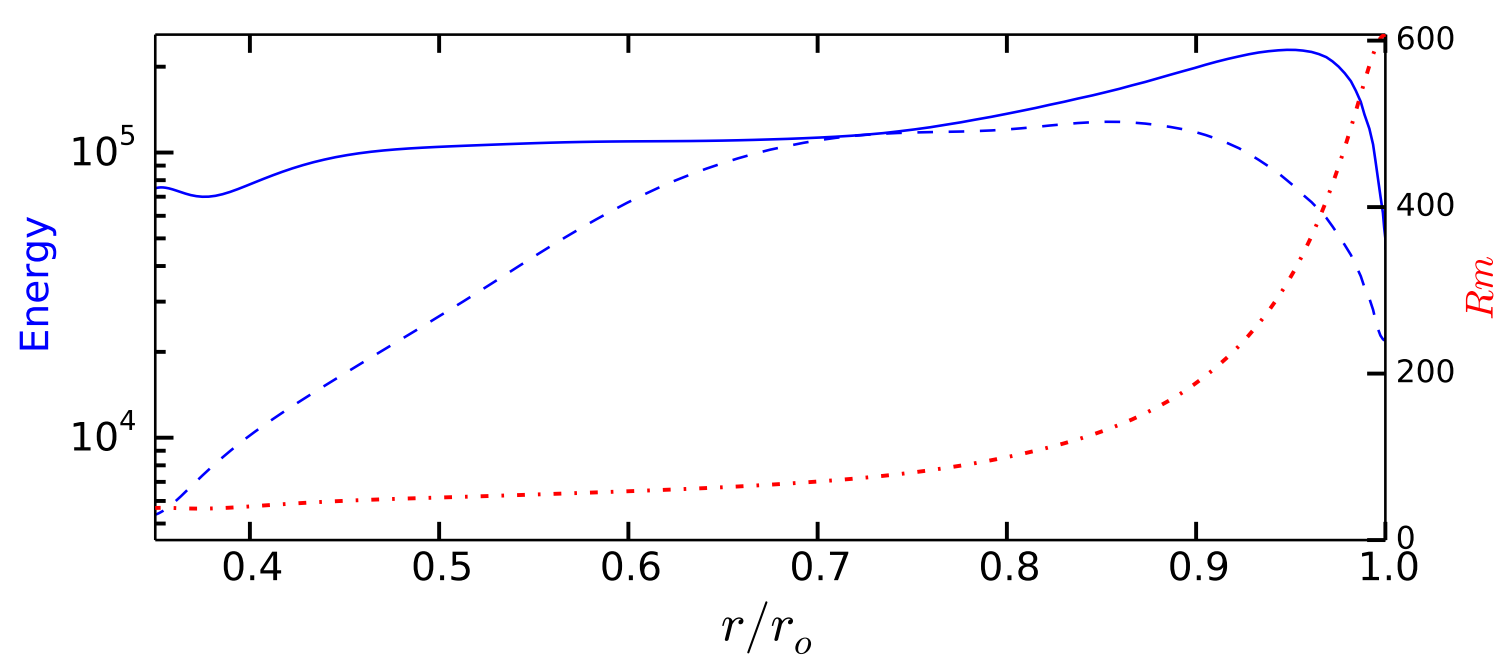

Figure 5.16: Similar to Fig. 5.5, but for model NA-Pr1-N6. 


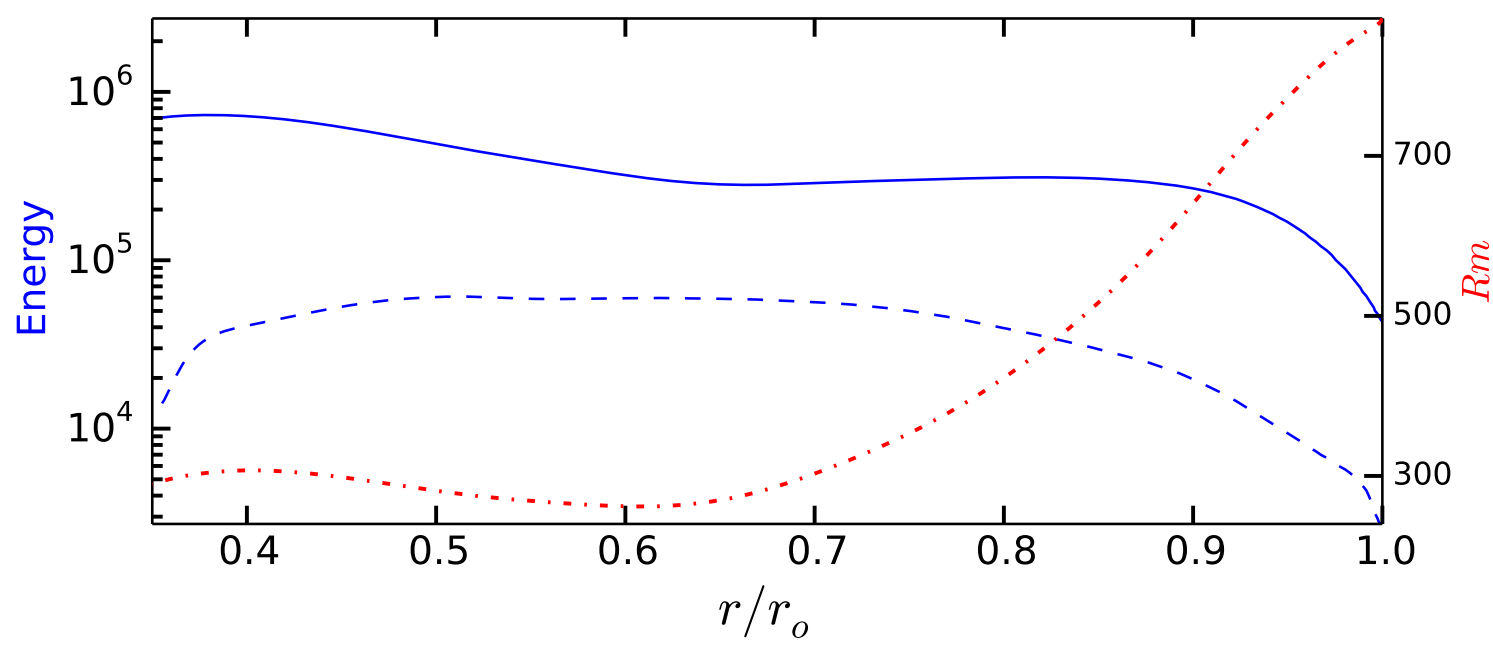

Figure 5.17: Similar to Fig. 5.5, but for model S-Pr1-N5.

Relatively large polar spots were found in a model where the magnetic field had an axial-dipole dominated geometry. In this case, moderate-sized magnetic flux concentrations were frequently formed. Occasionally, the coalescence of several such flux patches formed a large spot. The larger polar spots were also rather stable and persisted for many tens of rotations. The magnetic flux-bundles associated with the large dark spots were rooted in deeper helical columns. We conjecture that such anchored flux-bundles impose a long length scale and longer time-scale on the dark spots associated with them. This scenario is somewhat similar to the one put forth by Kitiashvili et al. (2010), who proposed that deeper simulation boxes, where larger and slower convection cells coexist with more turbulent ones, are important for having stable and large magnetic features.

The temporal evolution of the dark spots generated on the outer surface of the model was very rich. However, blurring the details of the model and analysing only the largescale modulations hides this temporal behaviour. Filtering of data in this way produces broad dark regions near the poles that maintain their integrity for hundreds of rotations. This feature agrees with observations (Hussain 2002) that indicate that polar dark spots persist for hundreds of stellar rotations. Low-pass filtering of our data also produces lowcontrast dark patches all over the surface that do not represent distinct dark spots, but seem to reflect large-scale heat flux inhomogeneities induced by a mesoscale convective network underneath the surface (Rincon et al. 2005, Bessolaz and Brun 2011). It is uncertain, however, whether such large scale inhomogeneities (not associated with dark spots) will survive turbulent photospheric convection, which we have not modelled.

Synthetic light curves of the model with polar spots showed variations with amplitudes that reached about $1.5 \%$. Comparing light curves at different inclinations suggests that dark spots at high latitudes in the simulation significantly contribute to the light curve modulations. We did not model the upper stellar layer below the photosphere and omitted the uppermost $\approx 5 \%$ of the stellar radius. However, the lateral extent of the large polar spot is much more than the thickness of the unmodelled layer, and the strong magnetic field in the dark spot is expected to highly quench convection in this omitted layer as well. 

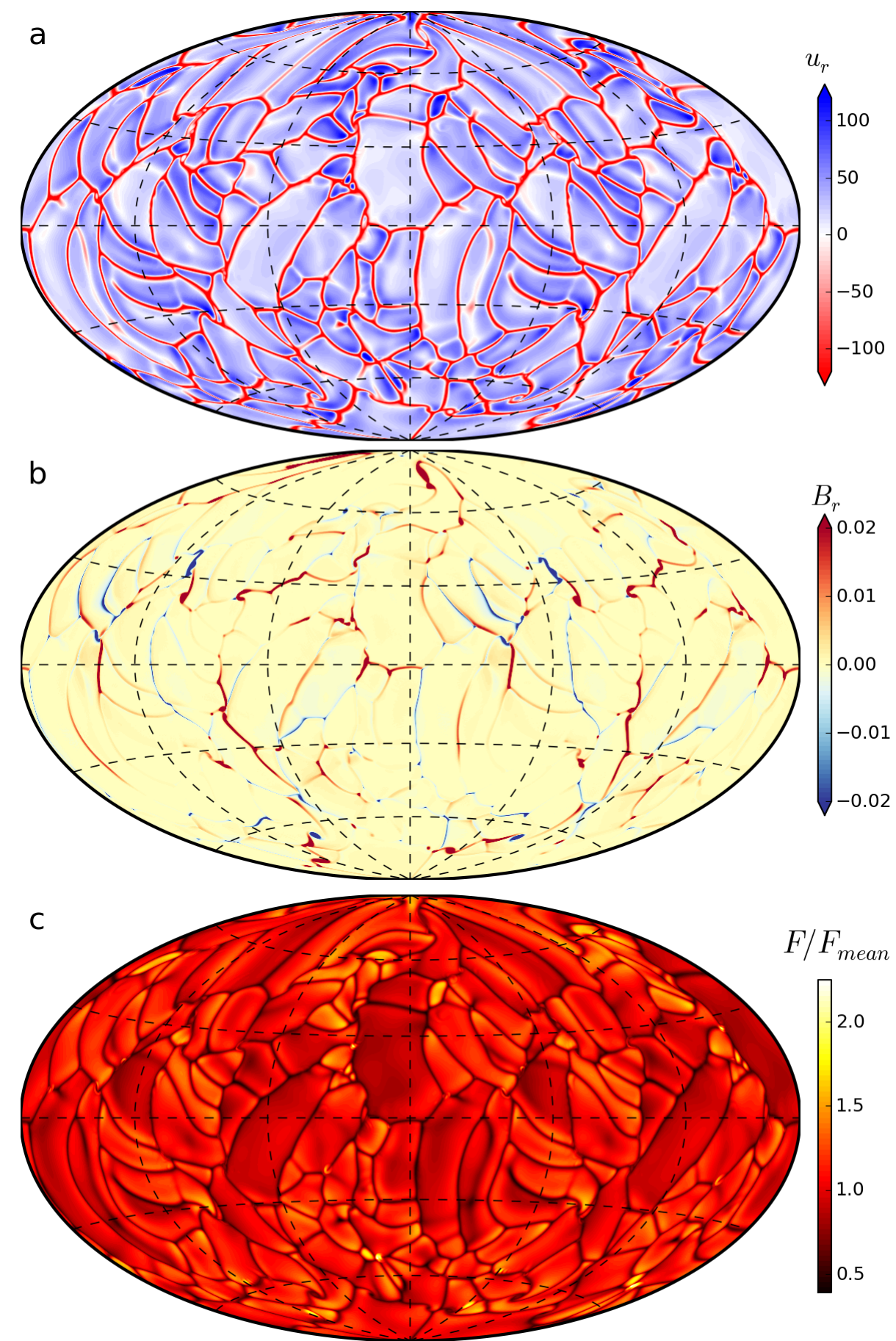

Figure 5.18: Similar to Fig. 5.3, but for model S-Pr1-N5. 
Therefore, we expect that the heat flux deficit in the large spot on the surface of our model represents that at the photosphere.

Based on a small parameter study, the following ingredients seem to be crucial for sizeable dark spot formation: 1) rotation-dominated convection, which favours the generation of a large-scale dynamo, 2) high-density stratification (five or more density scale heights) in the convection zone, which produces cellular convection along with deeper large-scale columns, and 3) dynamos with an axial-dipole-dominated field, which inherently produces a strong and stable magnetic field, allowing dark spots to grow to larger sizes.

Our results are based on a non-dimensional formulation of the anelastic-MHD equations. In a generic sense, they can be applied to different types of stars as long as rotation plays an important role. Examples include young pre-main sequence stars, evolved giants, or low-mass stars with small radiative cores. However, it can also be instructive to express our results in an exemplary way in physical units. We scaled the model with the large polar spot (A-Pr10-N5) to a pre-main sequence star with 0.7 solar masses and an effective surface temperature of $4200 \mathrm{~K}$ (spectral type K5). For the internal structure of this object we used results from a stellar evolution model (Granzer et al. 2000). We determined physical values at a radius $R_{o}$ somewhat below the photosphere (at about 0.96 $\mathrm{R}$ ) in the stellar model where the density has dropped by a factor of 150 below its value at $0.34 \mathrm{R}$. Using the physical values from the interior model and matching the heat flux at $R_{o}$ to the outer boundary heat flux of the polar spot case provides $v=4.3 \times 10^{8} \mathrm{~m}^{2} \mathrm{~s}^{-1}$, $\lambda=\kappa=4.3 \times 10^{7} \mathrm{~m}^{2} \mathrm{~s}^{-1}$ and $\Omega=5.6 \times 10^{-6} \mathrm{~s}^{-1}$ for the rotation rate. As usual for such simulations, the diffusivities are much higher than molecular values and must be understood as effective turbulent diffusivities. The rotation period is 13 days. This is not a very fast rotation rate, but from a force balance argument a system qualifies as rapidly rotating when the Rossby number is much lower than one, which is the case in our model. With these physical inputs, the variation range for the magnetic field in Fig. 5.3(d) is about $\pm 15 \mathrm{kG}$. The unsigned surface radial field averages to about $1.4 \mathrm{kG}$, which is a typical magnetic field strength found at the surface of rapidly rotating stars (Rossby number $<0.1$ ) (Reiners et al. 2009, Vidotto et al. 2014). Assuming that the heat flux variations (at least those associated with large spots) produced on the outer boundary of our simulation propagate to the stellar photosphere, we calculated from the Stefan-Boltzmann law an effective surface temperature that varied in the range $3000 \mathrm{~K}<T_{\text {eff }}<5000 \mathrm{~K}$ over the stellar surface. Considering only the large-scale component of the heat flux variations (as shown in Fig. 5.10(b)) narrows this range to $3700 \mathrm{~K}<T_{\text {eff }}<4400 \mathrm{~K}$. The temperature anomaly of the order $500 \mathrm{~K}$ is similar to what has been inferred observationally for stars compatible with the spectral type chosen here (Berdyugina 2005).

In stellar convection zones most of the heat is transported by vigorous convective motions. Our models are not in such a turbulent state, and a substantial amount of heat in the models is carried by diffusive processes. The magnetic quenching of convection is strong in our simulations, producing large variations in the heat flux carried by convection. However, the associated modulations in the total heat flux are somewhat moderate, reaching about $60 \%$ below surface average in the darkest spots. Hence, the range of temperature modulations mentioned above (based on heat flux variations) should be considered as lower estimates.

We have omitted many ingredients in our models that can affect the results. A sub- 
adiabatic radiative core might introduce additional interesting dynamics (Brown et al. 2010, Ghizaru et al. 2010, Masada et al. 2013). Including a sub-adiabatic coronal region might promote local bipolar structures (Warnecke et al. 2013). It is unclear how a photospheric small-scale dynamo (Vögler and Schüssler 2007) might affect the dark spots produced by an interior large-scale dynamo. A multitude of interesting phenomena such as formation of plages, which are related to the modelling of opacity, might change the nature of the light-curves we calculated. Furthermore, following up on the strategies outlined here using fully compressible approaches (e.g. Käpylä et al. 2012) would help to further confirm the starspot formation mechanism presented here.

The choice of a relatively large (magnetic) Prandtl number in the polar spot case can be debated. The need for high values of $P r$ and $P m$ to stabilize the ADD magnetic field is probably a consequence of using high values of the Ekman number in density-stratified stellar dynamo simulations (due to technical constraints). Similar to the planetary dynamo simulations, it should also be possible to find ADD dynamos in stellar dynamo simulations at low $\mathrm{Pr}$ and $\mathrm{Pm}$ when the Ekman number is low enough.

Our model is simplified in several respects and cannot address many of the details of the formation and the properties of starspots. Nonetheless, it is to our knowledge the first global model that generates rather large polar spots in a completely self-consistent way. It points at an interesting alternative to the flux-tube model of spot formation by a distributed dynamo mechanism.

\section{Acknowledgements}

We thank the referee for a very constructive review and Manfred Schüssler, Robert Cameron, and Jörn Warnecke for commenting on the manuscript. We also thank Jayant Joshi, Julien Morin, and Laurène Jouve for interesting discussions. Funding from the DFG through SFB 963/A17 and SPP 1488 is acknowledged. Computations were performed on RZG, HLRN (project "nip00031"), and the GWDG. The diligent efforts by Tilman Dannert (RZG), who implemented MPI and then hybrid OpenMP+MPI parallelisation in MagIC, are greatly appreciated. 



\section{Bibliography}

Aubert, J. (2005). Steady zonal flows in spherical shell dynamos. J. Flu. Mech., 542(1), 53-67.

Basri, G., Walkowicz, L. M., and Reiners, A. (2013). Comparison of kepler photometric variability with the sun on different timescales. ApJ, 769(1), 37.

Berdyugina, S. V. (2005). Starspots: A key to the stellar dynamo. Liv. Rev. Sol. Phys., 2(8).

Bessolaz, N. and Brun, A. S. (2011). Hunting for giant cells in deep stellar convective zones using wavelet analysis. ApJ, 728(2), 115.

Braginsky, S. I. and Roberts, P. H. (1995). Equations governing convection in earth's core and the geodynamo. Geophys. Astrophys. Fluid Dyn., 79(1-4), 1-97.

Brandenburg, A. (2005). The case for a distributed solar dynamo shaped by near-surface shear. ApJ, 625(1), 539.

Brandenburg, A., Kemel, K., Kleeorin, N., Mitra, D., and Rogachevskii, I. (2011). Detection of negative effective magnetic pressure instability in turbulence simulations. ApJL, 740(2), L50.

Brown, B. P., Browning, M. K., Brun, A. S., Miesch, M. S., and Toomre, J. (2010). Persistent magnetic wreaths in a rapidly rotating sun. ApJ, 711(1), 424.

Browning, M. K. (2008). Simulations of dynamo action in fully convective stars. ApJ, 676(2), 1262-1280.

Browning, M. K., Miesch, M. S., Brun, A. S., and Toomre, J. (2006). Dynamo action in the solar convection zone and tachocline: pumping and organization of toroidal fields. ApJL, 648(2), L157.

Busse, F. (1983). A model of mean zonal flows in the major planets. Geophys. Astrophys. Fluid Dyn., 23(2), 153-174.

Busse, F. H. and Simitev, R. D. (2006). Parameter dependences of convection-driven dynamos in rotating spherical fluid shells. Geophys. Astrophys. Fluid Dyn., 100(4-5), $341-361$.

Chabrier, G. and Küker, M. (2006). Large-scale $\alpha^{2}$-dynamo in low-mass stars and brown dwarfs. AEFA, 446, 1027. 
Charbonneau, P. (2005). Dynamo models of the solar cycle. Liv. Rev. Sol. Phys., 2(2).

Cheung, M., Rempel, M., Schüssler, M., et al. (2010). Simulation of the formation of a solar active region. ApJ, $\mathbf{7 2 0}(1), 233$.

Christensen, U. R. (2002). Zonal flow driven by strongly supercritical convection in rotating spherical shells. J. Flu. Mech., 470, 115-133.

Christensen, U. R. (2010). Dynamo scaling laws and applications to the planets. Spa. Sci. Rev., 152, 565-590.

Christensen, U. R. and Aubert, J. (2006). Scaling properties of convection-driven dynamos in rotating spherical shells and application to planetary magnetic fields. Geophys. J. Int., 166(1), 97-114.

Christensen, U. R. and Wicht, J. (2007). Numerical dynamo simulations. In G. Schubert, editor, Treatise of Geophysics, Vol. 8. Elsevier, Amsterdam.

Christensen, U. R., Holzwarth, V., and Reiners, A. (2009). Energy flux determines magnetic field strength of planets and stars. Nature, 457, 167.

Cole, E., Käpylä, P. J., Mantere, M. J., and Brandenburg, A. (2014). An azimuthal dynamo wave in spherical shell convection. ApJL, 780(2), L22.

Davenport, J. R., Hebb, L., and Hawley, S. L. (2014). Using transiting planets to model starspot evolution. arXiv:1408.5201.

Dobler, W., Stix, M., and Brandenburg, A. (2006). Magnetic field generation in fully convective rotating spheres. ApJ, 638(1), 336-347.

Donati, J.-F. and Landstreet, J. D. (2009). Magnetic fields of nondegenerate stars. Annu. Rev. Astron. Astrophys., 47, 333-370.

Dorch, S. (2004). Magnetic activity in late-type giant stars: Numerical mhd simulations of non-linear dynamo action in betelgeuse. AEFA, 423, 1101-1107.

Duarte, L. D., Gastine, T., and Wicht, J. (2013). Anelastic dynamo models with variable electrical conductivity: An application to gas giants. Phys. Earth Plan. Inter, 222(0), $22-34$.

Fan, Y. and Fang, F. (2014). A simulation of convective dynamo in the solar convective envelope: Maintenance of the solar-like differential rotation and emerging flux. ApJ, 789(1), 35 .

Featherstone, N. A., Browning, M. K., Brun, A. S., and Toomre, J. (2009). Effects of fossil magnetic fields on convective core dynamos in a-type stars. ApJ, 705(1), 1000.

Ferraro, V. (1937). The non-uniform rotation of the sun and its magnetic field. MNRAS, 97, 458-472.

Gastine, T. and Wicht, J. (2012). Effects of compressibility on driving zonal flow in gas giants. Icarus, 219(1), $428-442$. 
Gastine, T., Duarte, L., and Wicht, J. (2012). Dipolar versus multipolar dynamos: the influence of the background density stratification. $A \mathcal{E} A, \mathbf{5 4 6}, \mathrm{A} 19$.

Gastine, T., Morin, J., Duarte, L., Reiners, A., Christensen, U., and Wicht, J. (2013a).

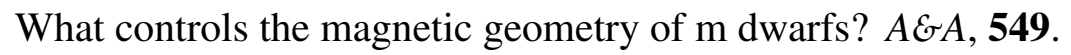

Gastine, T., Wicht, J., and Aurnou, J. (2013b). Zonal flow regimes in rotating anelastic spherical shells: An application to giant planets. Icarus, 225(1), 156-172.

Gastine, T., Yadav, R. K., Morin, J., Reiners, A., and Wicht, J. (2014). From solar-like to antisolar differential rotation in cool stars. MNRAS Let., 438(1), L76-L80.

Ghizaru, M., Charbonneau, P., and Smolarkiewicz, P. K. (2010). Magnetic cycles in global large-eddy simulations of solar convection. ApJL, 715(2), L133-L137.

Gilman, P. A. (1975). Linear simulations of boussinesq convection in a deep rotating spherical shell. J. Atm. Sci., 32(7), 1331-1352.

Gilman, P. A. (1977). Nonlinear dynamics of boussinesq convection in a deep rotating spherical shell-i. Geophys. Astrophys. Fluid Dyn., 8(1), 93-135.

Glatzmaier, G. A. (1984). Numerical simulations of stellar convective dynamos. I. The model and method. J. Comp. Phys., 55(3), 461-484.

Goudard, L. and Dormy, E. (2008). Relations between the dynamo region geometry and the magnetic behavior of stars and planets. Euro. Phy. Let., 83(5), 59001.

Granzer, T., Schüssler, M., Caligari, P., and Strassmeier, K. G. (2000). Distribution of starspots on cool stars. II. Pre-main-sequence and ZAMS stars between $0.4 M_{\text {sun }}$ and $1.7 M_{\text {sun }}$. AE A, 355, 1087-1097.

Grote, E. and Busse, F. (2000). Hemispherical dynamos generated by convection in rotating spherical shells. Phy. Rev. E, 62(3), 4457.

Holzwarth, V. (2004). Spot signatures of a solar-type dynamo in close binary stars. Astron. Nach., 325(5), 408-412.

Holzwarth, V., Mackay, D. H., and Jardine, M. (2006). The impact of meridional circulation on stellar butterfly diagrams and polar caps. MNRAS, 369(4), 1703-1718.

Hotta, H., Rempel, M., and Yokoyama, T. (2014). High-resolution calculations of the solar global convection with the reduced speed of sound technique. i. the structure of the convection and the magnetic field without the rotation. ApJ, 786(1), 24.

Hussain, G. (2002). Starspot lifetimes. Astronom. Nach., 323(3-4), 349-356.

Işik, E., Schüssler, M., and Solanki, S. (2007). Magnetic flux transport on active cool stars and starspot lifetimes. AEA A, 464, 1049-1057.

Işik, E., Schmitt, D., and Schüssler, M. (2011). Magnetic flux generation and transport in cool stars. AEFA, 528, A135. 
Jones, C. A. (2011). Planetary magnetic fields and fluid dynamos. Ann. Rev. Flu. Mech., 43(1), 583-614.

Jones, C. A., Boronski, P., Brun, A. S., Glatzmaier, G., Gastine, T., Miesch, M., and Wicht, J. (2011). Anelastic convection-driven dynamo benchmarks. Icarus, 216(1), $120-135$.

Julien, K., Rubio, A., Grooms, I., and Knobloch, E. (2012). Statistical and physical balances in low rossby number rayleigh-bénard convection. Geophys. E Astrophys. Flu. Dyn., 106(4-5), 392-428.

Käpylä, P. J., Korpi, M., Brandenburg, A., Mitra, D., and Tavakol, R. (2010). Convective dynamos in spherical wedge geometry. $A N$, 331(1), 73-81.

Käpylä, P. J., Mantere, M. J., and Brandenburg, A. (2012). Cyclic magnetic activity due to turbulent convection in spherical wedge geometry. ApJL, 755(1), L22.

Käpylä, P. J., Mantere, M. J., Cole, E., Warnecke, J., and Brandenburg, A. (2013). Effects of enhanced stratification on equatorward dynamo wave propagation. ApJ, 778(1), 41.

Kitiashvili, I., Kosovichev, A., Wray, A., and Mansour, N. (2010). Mechanism of spontaneous formation of stable magnetic structures on the sun. ApJ, 719(1), 307.

Lantz, S. and Fan, Y. (1999). Anelastic magnetohydrodynamic equations for modeling solar and stellar convection zones. ApJS, 121(1), 247-264.

Masada, Y., Yamada, K., and Kageyama, A. (2013). Effects of penetrative convection on solar dynamo. ApJ, 778(1), 11.

Miesch, M. S. (2005). Large-scale dynamics of the convection zone and tachocline. Liv. Rev. Sol. Phys., 2(1).

Mitra, D., Brandenburg, A., Kleeorin, N., and Rogachevskii, I. (2014). Intense bipolar structures from stratified helical dynamos. MNRAS, 445(1), 761-769.

Nelson, N. J., Brown, B. P., Brun, A. S., Miesch, M. S., and Toomre, J. (2013). Magnetic wreaths and cycles in convective dynamos. ApJ, 762(2), 73.

Olson, P., Christensen, U., and Glatzmaier, G. A. (1999). Numerical modeling of the geodynamo: mechanisms of field generation and equilibration. J. Geophys. Res., 104(B5), 10383-10404.

Pizzolato, N., Maggio, A., Micela, G., Sciortino, S., and Ventura, P. (2003). The stellar activity-rotation relationship revisited: Dependence of saturated and non-saturated $\mathrm{x}-$ ray emission regimes on stellar mass for late-type dwarfs. $A \mathcal{E} A$, 397(1), 147-158.

Proctor, M. R. E. and Weiss, N. O. (1982). Magnetoconvection. Rep. Prog. Phys., 45(11), 1317.

Reiners, A. (2012). Observations of cool-star magnetic fields. Liv. Rev. Sol. Phys., 9(1). 
Reiners, A., Basri, G., and Browning, M. (2009). Evidence for magnetic flux saturation in rapidly rotating $\mathrm{m}$ stars. $A p J, \mathbf{6 9 2}(1), 538$.

Reinhold, T., Reiners, A., and Basri, G. (2013). Rotation and differential rotation of active Kepler stars. AEA, 560, A4.

Rempel, M., Schüssler, M., and Knölker, M. (2009). Radiative magnetohydrodynamic simulation of sunspot structure. ApJ, 691(1), 640-649.

Rincon, F., Ligniéres, F., and Rieutord, M. (2005). Mesoscale flows in large aspect ratio simulations of turbulent compressible convection. $A \mathcal{E} A, \mathbf{4 3 0}(3)$, L57-L60.

Roberts, P. H. and Glatzmaier, G. A. (2000). Geodynamo theory and simulations. Rev. Mod. Phys., 72, 1081-1123.

Rogachevskii, I. and Kleeorin, N. (2007). Magnetic fluctuations and formation of largescale inhomogeneous magnetic structures in a turbulent convection. Phy. Rev. E, 76(5), 056307.

Sasaki, Y., Takehiro, S.-i., Kuramoto, K., and Hayashi, Y.-Y. (2011). Weak-field dynamo emerging in a rotating spherical shell with stress-free top and no-slip bottom boundaries. Phys. Earth Planet. Int., 188(3-4), 203-213.

Schrijver, C. J. and Title, A. M. (2001). On the formation of polar spots in sun-like stars. ApJ, 551(2), 1099-1106.

Schrinner, M., Petitdemange, L., and Dormy, E. (2011). Oscillatory dynamos and their induction mechanisms. AEFA, 530, 140.

Schrinner, M., Petitdemange, L., and Dormy, E. (2012). Dipole collapse and dynamo waves in global direct numerical simulations. ApJ, 752(2), 121.

Schrinner, M., Petitdemange, L., Raynaud, R., and Dormy, E. (2014). Topology and field strength in spherical, anelastic dynamo simulations. $A \mathcal{E} A, \mathbf{5 6 4}, \mathrm{A} 78$.

Schüssler, M. and Solanki, S. (1992). Why rapid rotators have polar spots. $A \mathcal{E} A, \mathbf{2 6 4}$, L13-L16.

Simitev, R. D. and Busse, F. (2005). Prandtl-number dependence of convection-driven dynamos in rotating spherical fluid shells. J. Flu. Mech., 532, 365-388.

Simitev, R. D. and Busse, F. H. (2009). Bistability and hysteresis of dipolar dynamos generated by turbulent convection in rotating spherical shells. Europhys. Lett., 85(1), 19001.

Sreenivasan, B. and Jones, C. A. (2006). The role of inertia in the evolution of spherical dynamos. Geophys. J. Int., 164(2), 467-476.

Steffen, M. and Freytag, B. (2007). Rotating 'star-in-a-box' experiments. Astron. Nach., 328(10), 1054-1058. 
Stein, R. and Nordlund, Å. (2006). Solar small-scale magnetoconvection. ApJ, 642(2), $1246-1255$.

Stein, R., Lagerfjärd, A., Nordlund, Å., and Georgobiani, D. (2011). Solar flux emergence simulations. Sol. Phys., 268(2), 271-282.

Stein, R. F. (2012). Solar surface magneto-convection. Liv. Rev. Sol. Phys., 9(4).

Strassmeier, K. (2002). Doppler images of starspots. Astron. Nach., 323(3-4), 309-316.

Strassmeier, K. G. (2009). Starspots. AEAA Rev., 17(3), 251-308.

Vidotto, A., Gregory, S., Jardine, M., Donati, J., Petit, P., Morin, J., Folsom, C., Bouvier, J., Cameron, A., Hussain, G., et al. (2014). Stellar magnetism: empirical trends with age and rotation. MNRAS, 441(3), 2361-2374.

Vögler, A. and Schüssler, M. (2007). A solar surface dynamo. AEFA, 465(3).

Vögler, A., Shelyag, S., Schüssler, M., Cattaneo, F., Emonet, T., and Linde, T. (2005). Simulations of magneto-convection in the solar photosphere. AEEA, 429(1), 335-351.

Vogt, S. S., Penrod, G. D., and Hatzes, A. P. (1987). Doppler images of rotating stars using maximum entropy image reconstruction. ApJ, 321, 496-515.

Warnecke, J., Losada, I. R., Brandenburg, A., Kleeorin, N., and Rogachevskii, I. (2013). Bipolar magnetic structures driven by stratified turbulence with a coronal envelope. ApJL, 777(2), L37.

Wicht, J. (2002). Inner-core conductivity in numerical dynamo simulations. Phys. Earth Planet. Int., 132, 281-302.

Yadav, R. K., Gastine, T., Christensen, U. R., and Duarte, L. D. V. (2013a). Consistent scaling laws in anelastic spherical shell dynamos. ApJ, 774(1), 6 .

Yadav, R. K., Gastine, T., and Christensen, U. R. (2013b). Scaling laws in spherical shell dynamos with free-slip boundaries. Icarus, 225(1), 185 - 193.

Zaqarashvili, T., Oliver, R., Ballester, J., Carbonell, M., Khodachenko, M., Lammer, H., Leitzinger, M., and Odert, P. (2011). Rossby waves and polar spots in rapidly rotating stars: implications for stellar wind evolution. $A \mathcal{E} A, \mathbf{5 3 2}, 139$. 


\section{Conclusions and outlook}

In this thesis I used direct numerical simulations of dynamos in rapidly rotating spherical shells to analyse the magnetic field generation processes in the interior of gas planet and low-mass stars. The first focus was to use a collection of progressively complex models to analyse the scaling behaviour of important volumetrically-averaged mean quantities (mean velocity and mean magnetic field). With these studies I showed that the power-based scaling laws proposed by Christensen and collaborators are remarkably robust across a wide range of numerical models. This explains why scaling laws inferred from relatively simple Boussinesq simulations were already able to predict the magnetic field in Jupiter and some rapidly rotating stars to a good extent.

Similar to the earlier studies I also found that the exact values of the transport coefficients controlling the diffusion processes (viscous, thermal, electrical) were influencing the scaling laws in numerical simulations, although the influence was small. Nonetheless, if one extrapolates the scaling laws to natural objects and compare the predictions with observations, this small effect of diffusive parameters observed in numerical scaling laws produces a rather large discrepancy. Hence, it has been argued that to first-order numerical dynamo simulations are capturing the relevant physics, but we are yet to reach a true natural state where various diffusion processes can be considered negligible. With this argument in mind the dependence of diffusive parameters is deliberately dropped from the numerical scaling laws. As of now, this practice is not completely justifiable and we need to carry out a parameter study of dynamos with much lower viscosity in the future to support of refute it.

In the context of scaling law studies I was not able to answer an important question: what is the relation between magnetic field observed on the surface of a simulation to the magnetic field in the convective interior of the simulation, and how do various control parameters affect it? The scaling laws derived from the numerical models usually contain volumetrically averaged mean properties. While, in the observational context, this is not the case and one observes the magnetic field away from the region where it is being generated. The most drastic assumption goes into assuming the magnitude of the toroidal magnetic field, most of which is inaccessible to observations. We need to analyse our numerical models thoroughly and figure out a robust connection between the surface field strength and the field inside the bulk of the convection zone. This will help us to put the predictions from numerical scaling laws and the observations on the same platform.

In regards to the solar-like/anti-solar differential rotation, main developments are expected from the observational viewpoint. The plethora of numerical models studied here all strongly support the existence of anti-solar differential rotation in stars, much like what we see on the ice-giant planets (Uranus and Neptune). There have been indications that some stars do show anti-solar differential rotation but the evidence remains tentative. 
More observational campaigns are disparately needed to constrain our understanding of the stellar differential rotation.

Finally, the high-resolution numerical simulations showed that it is indeed possible to self-consistently produce dark spots in global dynamo models. With these simulations I combined the conventionally disparate approaches of studying magnetic field generation in global geometries and dark spot formation in local geometries. These new simulations highlight the importance of density stratification and rapid-rotation in producing dark spots in global models. However, this study should be considered as a proof-of-concept and much work remains to be done. Due to the numerically-demanding nature of the simulations in this study a thorough parameter study was not possible. As we discussed earlier, diffusion processes are still affecting our numerical simulations. We need to quantify these effects by performing a systematic study of dark-spot producing simulations at different control parameters. Furthermore, the anelastic approximation does not allow us to model the outer few percent of the stellar convection zone. One way to bridge this gap is to use the output from these global simulations and feed sections of the output to the fully compressible box-simulations to see how the photospheric turbulent convection affects the magnetic field structures coming from the convection zone below it. 


\section{Publications}

\section{Refereed publications}

- R. K. Yadav, T. Gastine, and U. R. Christensen, "Scaling laws in spherical shell dynamos with free-slip boundaries", Icarus 225, 185, 2013.

- R. K. Yadav, T. Gastine, U. R. Christensen, and L. Duarte, "Consistent scaling laws in anelastic spherical shell dynamos", ApJ 774, 6, 2013.

- R. K. Yadav, T. Gastine, U. R. Christensen, and A. Reiners, "Formation of starspots in self-consistent global dynamo models: Polar spots on cool stars", A\&A 573, A68, 2015.

- T. Gastine, R. K. Yadav, J. Morin, A. Reiners, and J. Wicht, "From solar-like to anti-solar differential rotation in cool stars", MNRAS Let. 438, L76-L80, 2013.

\section{Conference Contributions}

- MHD days, Potsdam, Germany, Dec. 2-3, 2014.

Talk: Formation of starspots in self-consistent global dynamo models.

- CoolStars 18, Flagstaff, USA, June 9-13, 2014.

Talk + Poster + Proceeding: Spontaneous formation of dark polar spots in global dynamo simulations.

- Astrophysical Turbulence: From Galaxies to Planets, Dresden, Germany, October 7-11, 2013.

Talk: Bridging planets and stars in dynamo models: scaling laws for compressible spherical shell dynamos.

- Magnetic fields throughout stellar evolution (IAUS 302), Biarritz, France, Aug 2530, 2013.

Poster + Proceeding: Bridging planets and stars in dynamo models

- Int. Symp. on Geophysical and Astrophysical Dynamos, Ascona, Switzerland, July 7-12, 2013.

Talk: Bridging planets and stars in dynamo models: scaling laws for anelastic spherical shell dynamos 
- Rocks'n’Stars Conference, Göttingen, Germany, Oct 8-11, 2012. Poster: Scaling relations in dynamos with stress-free boundaries

- European GdR Dynamo and MHD days Meeting, Nice, France, October 1-4, 2012. Poster: Scaling relations in dynamos with stress-free boundaries 


\section{Acknowledgements}

First of all, I would like to thank Uli for giving me the opportunity to be a part of this wonderful institute. I have been very fortunate to have worked with him and receive some of his outpouring knowledge about anything dynamo. I also greatly appreciate his readiness to help me to go to various conferences where I have enjoyed a lot of good time and have made many friends. I will always remember his chill-attitude and many of his anecdotes that he shared.

Thomas has been a mentor, a collaborator, and a friend since the beginning. His competence and confidence have always inspired me to try harder. Without doubt, this thesis work would not have been possible without his help. Our conversations have always been interesting and fruitful. I am truly grateful to him for his everyday advise in science and everything else.

I am thankful to Ansgar for sharing his observational wisdom with me throughout my thesis. He was always there to answer my naive questions about what happens in reality. I also thank Johannes for his advise and encouragement. His joyful attitude has always assured me that things will be okay.

I thank my friend and collaborator Lúcia for so many things which I can't list here. In the early stages, she was the first person who had to endure my questions regarding my research. She has taught me the value of diligence and perseverance in science and in life.

I thank perpetually-humble late Dieter and our new energetic co-ordinator Sonja for making this solar system school the way it is. Without their untiring efforts I would not have even been here. I also thank our charming institute ladies Silvia, Petra, Ines, Claudia, and Saskia for their help at un-countable occasions.

I am thankful to Dr. Julien Aubert for accepting to be an external referee for my thesis, and to Prof. Dr. Andreas Tilgner, Prof. Dr. Wolfgang Glatzel, Prof. Dr. Manfred Schüssler, and Prof. Dr. Stefan Dreizler for agreeing to be in the examination committee.

I thank my friends Patricio (an awesome flat-mate!), Fabien, Domenico, and Feng for many delightful conversations. I also thank Fabien and Andrea for their prompt help with everything German. A big thank to all of them for being nice office mates and for responding to my intermittent queries ranging from science to which train to take.

Thanks to Julien, Jörn, Martin, Laurène, Jayant, Wieland, Robert, and Emanuele for many enlightening conversations and to Jayant, Megha, Nagaraju \& Latta, Sanjeev, Navdeep, Girjesh, Jisesh, Chaitanya, Gune, Gautum, Jan, and Ankit for their help and joyful discussions. A special thanks to Patrik and Emanuele for helping me to move from Lindau to Göttingen, and to Sonja, David, and Atefeh for organizing a nice retreat which was a very educational and pleasant experience.

I am grateful to my family for their love and encouragement, and for supporting my un-conventional (in my little town) endeavours despite the difficulties. Lastly, I thank the 
love of my life for putting sense in to my head, for her un-conditional love, and for being with me despite the distance. I would not be the kind person I am today without her. 


\section{Curriculum vitae}

\section{Personal}

- Name: Rakesh Kumar Yadav

- Date of birth: March 30, 1987

- Nationality: Indian

\section{Education}

- July 2006 - May 2011: Integrated M. Sc. in Physics (Bachelors + Masters) Indian institute of technology, Kanpur, India.

- April 2012 - January 2015: Doctorate University: Universität Göttingen, Germany

Institute: Max-Planck-Institut für Sonnensystemforschung, Göttingen, Germany

\section{Research Contributions}

- R. K. Yadav, T. Gastine, U. R. Christensen, and A. Reiners, "Formation of starspots in self-consistent global dynamo models: Polar spots on cool stars", A\&A 573, A68, 2015.

- T. Gastine, R. K. Yadav, J. Morin, A. Reiners, and J. Wicht, "From solar-like to anti-solar differential rotation in cool stars", MNRAS Let. 438, L76-L80, 2013.

- R. K. Yadav, T. Gastine, U. R. Christensen, and L. Duarte, “Consistent scaling laws in anelastic spherical shell dynamos", ApJ 774, 6, 2013.

- M. K. Verma, A. Chatterjee, K. S. Reddy, R. K. Yadav, S. Paul, M. Chandra, and R. Samtaney, "Benchmarking and scaling studies of a pseudospectral code Tarang for turbulence simulations", Pramana 81, 4, 2013.

- M. K. Verma and R. K. Yadav, "Supercriticality to subcriticality in dynamo transitions", Phy. Plasmas 20, 072307, 2013.

- R. K. Yadav, T. Gastine, and U. R. Christensen, "Scaling laws in spherical shell dynamos with free-slip boundaries", Icarus 225, 185, 2013. 
- R. K. Yadav, M. K. Verma, and P. wahi, "Bistability and chaos in the Taylor-Green dynamo", Phys. Rev. E 85, 036301, 2012.

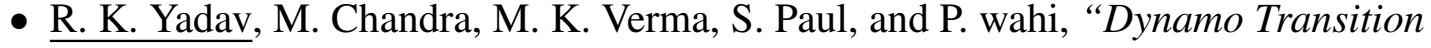
under Taylor-Green forcing", Europhysics Lett. 90, 69001, 2010. 